UNITED STATES DEPARTMENT OF THE INTERIOR GEOLOGICAL SURVEY

\author{
Processed Strong-Motion Records \\ Recorded on Bougainville Is iand, Papua New Guinea; \\ Earthquakes of December 13, 1981 and March 18, 1983 \\ by
}

B.L. Silverstein, A.G. Brady, and P.N. Mork

Menlo Park, California

February 1986

U.S. Geological Survey

Open-File Report 86-264

This report is preliminary and has not been reviewed for conformity with U.S. Geological Survey editorial standards. Any use of trade names is for descriptive purposes oniy and does not imply endorsement by the USGS. 
. 
CONTENTS

Page

Introduction

Cooperative efforts

Record description - 3

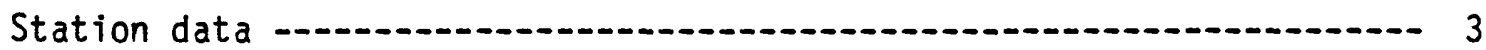

Seismological data

Current USGS processing- 4

Results/summary - 6

Acknow ledgments

References

Tables

Table 1. Source parameters for Solomon Is lands earthquakes and the New Ireland earthquake 8

2. Peak values of processed records 9

3. Long-period, low-frequency, filter parameters 10

\section{Illustrations}

Figure 1. Map showing location of Bougainville Is land 11

2. Map showing epicenters and strong-motion instrument locations

3. Copies of original records 13

Appendix I Computer plots 20

Appendix II Current lists of processed records 141 
CONTENTS (cont inued)

Station

Page No. for plots of six processing steps*

$\begin{array}{llllll}1 & 2 & 3 & 4 & 5 & 6\end{array}$

460 Bench, Panguna Mine

21

23

29

32

$35 \quad 38$

13 December, 1981; 0139 Gmt

Long., Up, Tran.

460 Bench, Panguna Mine

41

42

45

48

$51 \quad 54$

13 December, 1981; 1324 Gmt

Long., Up, Tran.

Arawa Town

57

$\begin{array}{lllll}69 & 105 & 114 & 123 & 132\end{array}$

18 March, 1983; 0905 Gmt

Long., Up, Tran.

Bato Bridge

81

108

117

$126 \quad 135$

18 March, 1983; $0905 \mathrm{Gmt}$

Long., Up, Tran.

BVE 80 (Panguna)

65

$$
93
$$

111

120

$129 \quad 138$

18 March, 1983; 0905 Gmt

Long., Up, Tran.

*Processing stages and plot format:

1) Uncorrected accelerogram

2) Corrected acceleration, velocity, displacement

3) Relative velocity response spectrum, linear plot

4) Response spectrum, tripartite $\log -\log$ plot

5) Fourier amplitude spectrum, linear plot

6) Fourier, amplitude spectrum, log-log plot

In column 1 each plot contains all three components. For columns 2 through 6 , the indicated page number refers to the first of three components for this record. 


\section{INTRODUCTION}

\section{U.S. Geological Survey National Permanent Network}

In a continuing effort to present processed strong-motion earthquake data to the engineering and seismological communities and the general public, the U.S. Geological Survey (USGS) maintains a nation-wide network of federally owned permanant strong-motion earthquake recorders and routinely processes and reports on records obtained from them. The network also includes instruments owned by other federal agencies, but maintained by the USGS. The National Strong-Motion program also occasionally produces joint reports with other agencies (see Cooperative Efforts).

The main purposes of these reports are to present the computer plots of corrected accelerograms and their spectra, and to provide sufficient information on the earthquakes, stations, records, and results so that the reader may make an informed decision whether further study is warranted. A list of completed reports is given in Appendix II. Digital data is available on tape from the National Geophysical Data Center (NGDC), NOAA, Mail Stop E/GC11, 325 Broadway, Boulder, Colorado 80303.

Note: This report supercedes Open File Report 85-261, Processed StrongMotion Records from the Solomon Islands Earthquakes of December 13, 1981 and March 18, 1983. The response spectra for the BVE records were corrected, the Arawa records were redone with a Butterworth filter a $0.20 \mathrm{~Hz}$ to match the other records of the same event, and a frequency domain filter with a transition band of $25-50 \mathrm{~Hz}$ was applied to eliminate high-frequency noise that sometimes occurs during the digitization of low-amplitude records. The latter filter replaces the standard time-domain method usually used. 


\section{Cooperative Efforts}

The permanent strong-motion network is managed by several projects within the Branch of Engineering Seismology and Geology of the USGS. The program and its predecessors have been involved with strong-motion recording and processing since the early 1930's. As procedures and techniques have improved within the areas of network planning, instrument maintenance, record processing, and associated research, the USGS has been able to exchange knowledge in cooperative efforts with other agencies in the field of strong-motion earthquake engineering and engineering seismology.

Cooperative efforts have included those with:

a) Federal agencies such as the Bureau of Reclamation, the Army Corps of Engineers, the Veterans Administration, the Federal Highways Administration, and others.

b) State agencies such as the California Division of Mines and Geology, the California Department of Water Resources, the Washington State Highways Administration, and others.

c) Universities such as the California Institute of Technology, the University of Southern California, Columbia Universtity, and others.

d) Foreign and domestic agencies sometimes jointly responsible for strong-motion networks including those in Fiji, Greece, Italy, the Soviet Union, Yugoslavia, and others. 
Record Description

The records processed in this report are from stations on Bougainville Island in Papua New Guinea, obtained from earthquakes in the Solomon Islands region of the southwest Pacific 0cean. This particular part of the major circum-Pacific plate bounderies does not have as high a seismic potential or probability of a great earthquake as other parts, but incomplete historical records might be a contributing factor to this assesment (McCann and others, 1979).

The records were provided by $K$. McKue formerly with Bougainville Copper Limited, Panguna, Bougainville, Papua New Guinea and currently with Seismology Section, Bureau of Mineral Resources, P.0. Box 378, Canberra A.C.T., Austra1ia. Two small events (M5-6) resulted in peaks of 2 percent $g, 4$ percent $g$, and 30 percent $g$ at three different sites having epicentral distances of about $270 \mathrm{~km}$. See table 3 for furthur record descriptions and details.

\title{
Station Data
}

\author{
Arawa Town 1/4g SMA-1 located on rock. Elevation $30 \mathrm{~m}$. \\ Bato Bridge 1/2g SMA-1 located on rock. Elevation $30 \mathrm{~m}$. \\ Bench (Panguna Mine) $1 / 2 \mathrm{~g}$ SMA-1 located on rock. \\ BVE 80 (Panguna) 1g SMA-1 located on volcanic ash over weathered \\ rock. (Elevation $300 \mathrm{~m}$ ).
}

Seismological Data

Figure 1 is a map showing the location of Bougainville Island. Figure 2 shows the epicenters and strong-motion accelerograph locations. 
The larqest of these earthquakes, a magnitude 7.9 (Ms) on March 18, 1983 was recorded by the Arawa Town, Bato Bridge and BVE 80 (Panquna) stations, located in the Arawa and Panguna areas on Bougainville Island (K. McKue, Bougainville Copper, written commun., October, 1983).

The two earthquakes on December 13, 1981, were recorded by the Bench station. These events were magnitude $5.9 \mathrm{Mb}$ (0139 Gmt) and $5.5 \mathrm{Mb}$. (1324 Gmt). Source data for all three events are listed in Table 1.

\section{DIGITIZATION AND PROCESSING}

\section{Current USGS Processing}

The current USGS steps for processing are:

1. A commercial digitizing firm (IOM-TOWILL in Santa Clara, California) digitizes the records on a trace-following, computer-controlled laser scanner. The data is digitized at unequal time intervals, although close to equispaced in time when clear of sharp peaks, at an average of 600 samples per second.

2. If more than $10-\mathrm{cm}$ of a record is digitized (film speed $=1 \mathrm{~cm} / \mathrm{s}$ ) the digitized portion is divided into approximately $10-\mathrm{cm}$ frames and each frame is digitized separately with $2.5 \mathrm{~cm}$ overlaps. The frames are reassembled using specially inserted vertical lines; the lines mark the end and/or beginning of each frame. Each vertical line is digitized twice, once in each adjacent frame, and then used in reassembling the record. 
3. The uncorrected data are prepared by subtracting the digitized reference traces from the data traces, and using the digitized time marks to determine the time scale, and subtracting the mean. The instrument sensitivities scale the ordinates to acceleration.

4. The data are passed through a correction algorithm that applies a high-frequency filter a $50 \mathrm{~Hz}$ low-pass filter instrument correction, base-line correction in the form of a low-frequency filter, and decimation to 200 sps. Plots of the corrected acceleration, velocity, and displacement for the three components of each record are included.

Initial selection of long-period filters is based on the convention of retaining a period content greater than or equal to the strong-motion duration of the records. The final Butterworth filter parameters are chosen to eliminate any visible serious noise content in the calculated displacements taking into account the guidelines described in Basili and Brady (1978).

In some instances a frequency domain filter with a transition band of $25-50 \mathrm{~Hz}$ is applied to eliminate high frequency noise that may occur during the digitization of low amplitude records. This latter procedure replaces the standard time-domain method usually used.

5. The maximum relative velocity response spectra are calculated for damping values of $0,2,5,10$, and 20 percent of critical. These response spectra are calculated for the period range that commences at $0.04 \mathrm{~s}$ and ends with the long period that corresponds to the lowfrequency filter limit used in the base-line correction algorithm. The dashed curve on this plot is the unsmoothed Fourier amplitude spectrum, calculated at the same periods as the response spectra. 
The second response spectrum plot is the pseudo-velocity response spectra, calculated for the same five damping values. This tripartite plot also has the values for the maximum relative displacement response spectrum as well as the pseudo-absolute acceleration spectrum.

6. Fourier amplitude spectra, calculated by FFT, are presented on linear and $\log -\log$ axes to accent the particular characteristics at each end of the spectrum.

For a more complete description of the processing method see Converse (1984).

\section{Results and Summary}

Reproduced in Appendix I are computer-generated plots that provide a vis= ual description of the recorded accelerations and their processed results. These plots may be used to measure specific earthquake characteristics or record parameters, and to select records for further study using the diqital data.

The unusually large differences between the digitized and corrected peak values of the Bench transverse traces, are due to the manual digitizing of isolated single peaks at those locations subsequently treated as high frequency triangular spikes and amplified during the instrument correction.

\section{ACKNOWLEDGMENTS}

Modern digital processing of strong-motion accelerograms has evolved over many years, from the work of Trifunic and Lee at $\mathrm{Cal}$ Tech and of Virgilio Perez at the USGS, to the present ongoing refinements of the process in April Converse's AGRAM series. The authors acknowledge these and other contributions to strong-motion processing. 


\section{REFERENCES}

Basili, M., and Brady, G., 1978, Low frequency filtering and the selection of limits for accelerogram corrections: Sixth Furopean Conference on Fnaineering Seismology, Dubrovnik, Yugoslavia, 8 p.

Converse, A.M., 1985, AGRAM: A series of computer programs for processing digitized strong-motion accelerograms, version 2.0: U.S. Geological Survey Open-Fil e Report 81-525, $118 \mathrm{p}$.

McCann, W.R., Nishenko, S.P., Sykes, L.R. and Krause, J., 1979, Seismic gaps and plate tectonics: seismic potential for major plate boundaries: Pure and Applied Geophysics, v.117, pp. 1082-1147. 
Table 1: Source Parameters for Solomon Islands Earthquakes and the New Ireland Earthquake*

1. Date $12 / 13 / 81$ (Solomon Islands) 2. Date 12/13/81 (Solomon Islands)

Time $013914.3 \mathrm{Gmt}$

Epicenter 6.387S, 154.929E

Time

$132417.3 \mathrm{Gmt}$

Depth

$50 \mathrm{~km}$

Epicenter

$6.343 S, 154.923 E$

Magnitude

$5.9 M_{B}, 6.0 M_{L^{+}}$

Depth

$48 \mathrm{~km}$

Magnitude

$5.5 \mathrm{M}_{B}, 5.7 \mathrm{M}_{L^{+}}$

3. Date 03/18/83 (New Ireland)

Time $\quad 090550.0 \mathrm{Gmt}$

Epicenter $\quad 4.833 S, 153.581 E$

Depth $\quad 89 \mathrm{~km}$

Magnitude

$7.9 \mathrm{M}_{\mathrm{s}}, 7.7 \mathrm{M}_{\mathrm{s}}{ }^{+}$.

* Unless otherwise noted, all seismological data in this report is taken from Preliminary Determination of Epicenters (PDE) published by the U.S. Geological Survey.

+ Kevin McCue, Bougainville Copper, written communication, Oct. 1983. 


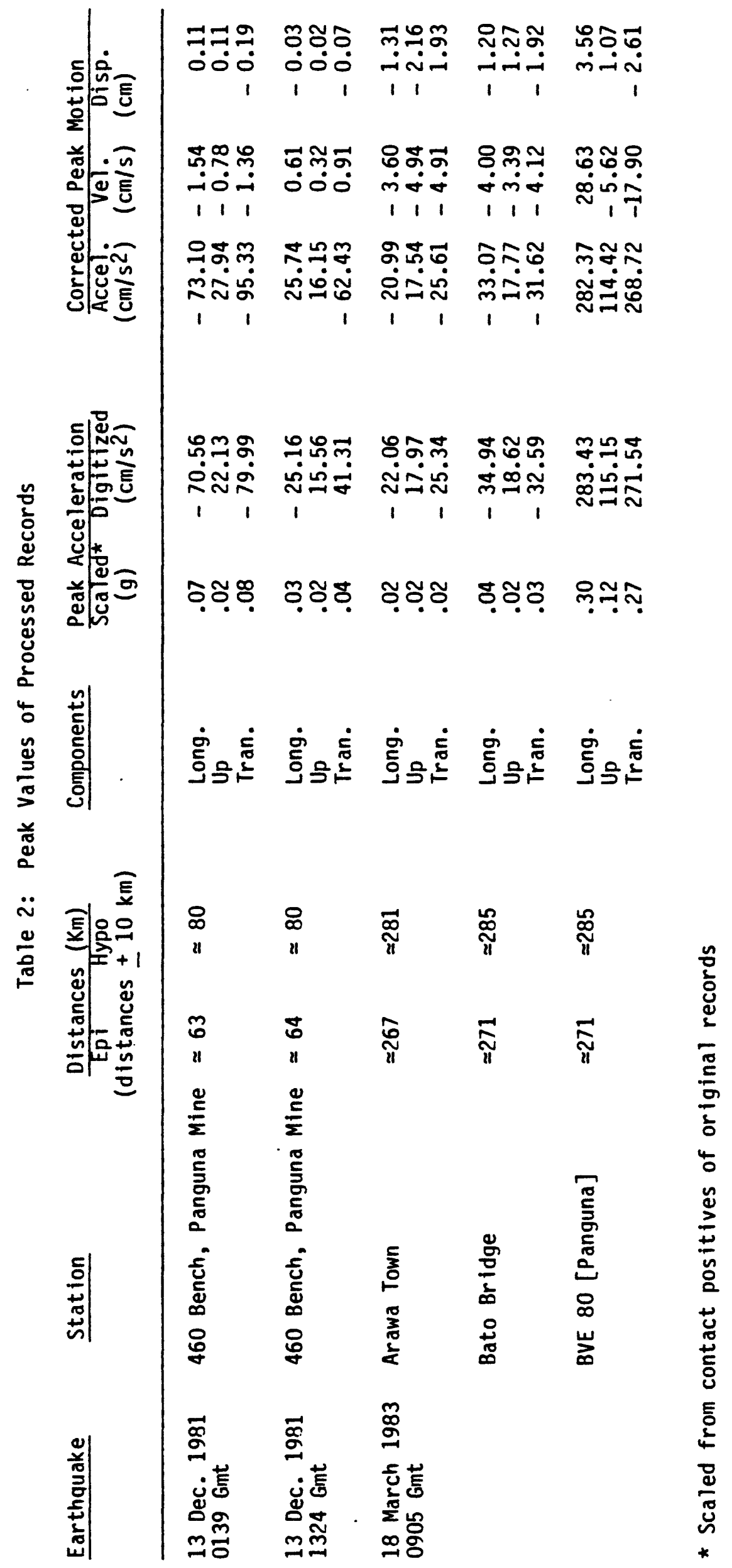


Table 3: Long-Period, Low Frequency, Filter Parameters

Final Original

Butterworth $(\mathrm{Hz})$ Order Butterworth $(\mathrm{Hz})$ Order

\begin{tabular}{lllll}
\hline Bench (0139) & 0.50 & 4 & 0.25 & 4 \\
Bench (1324) & 1.0 & 4 & 0.125 & 4 \\
Arawa Town & 0.20 & 4 & 0.083 & 4 \\
Bato Bridge & 0.20 & 4 & 0.125 & 4 \\
BVE 80 (Panguna Mine) & 0.20 & 4 & 0.125 & 4
\end{tabular}



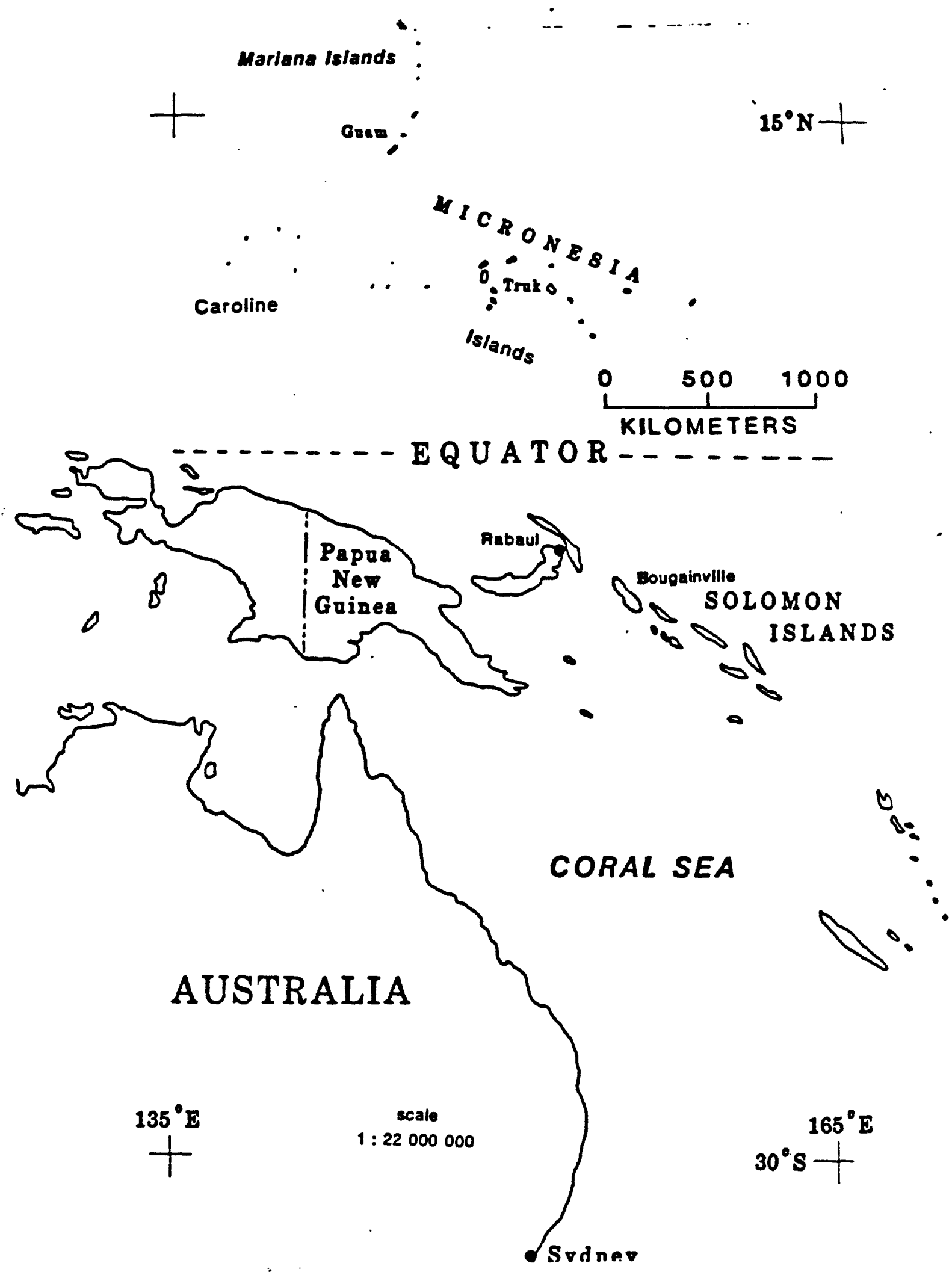

Figure 1: Map showing location of Bougainville Island. 


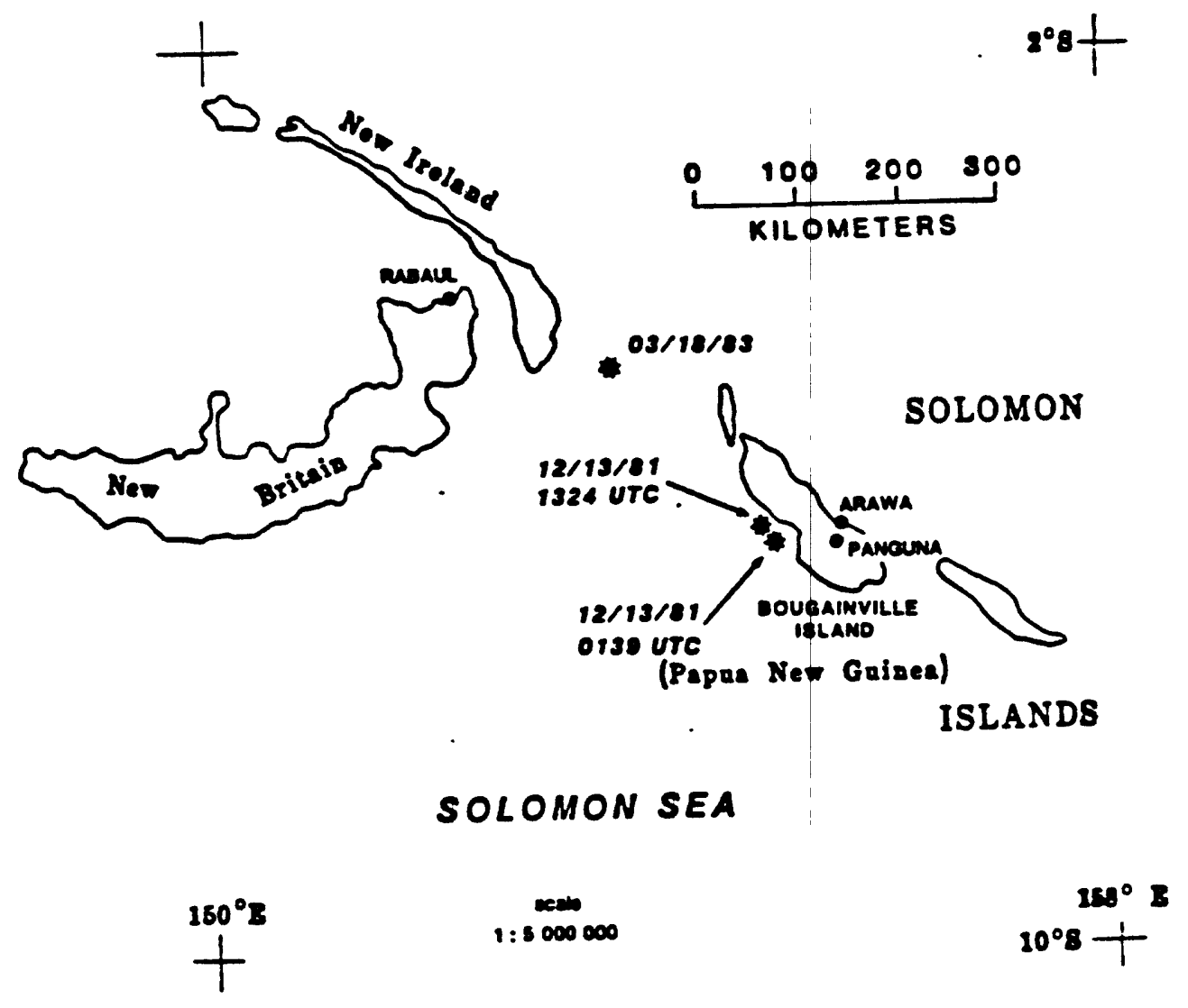

Figure 2: Map showing epicenters and strong-motion instrument locations. 

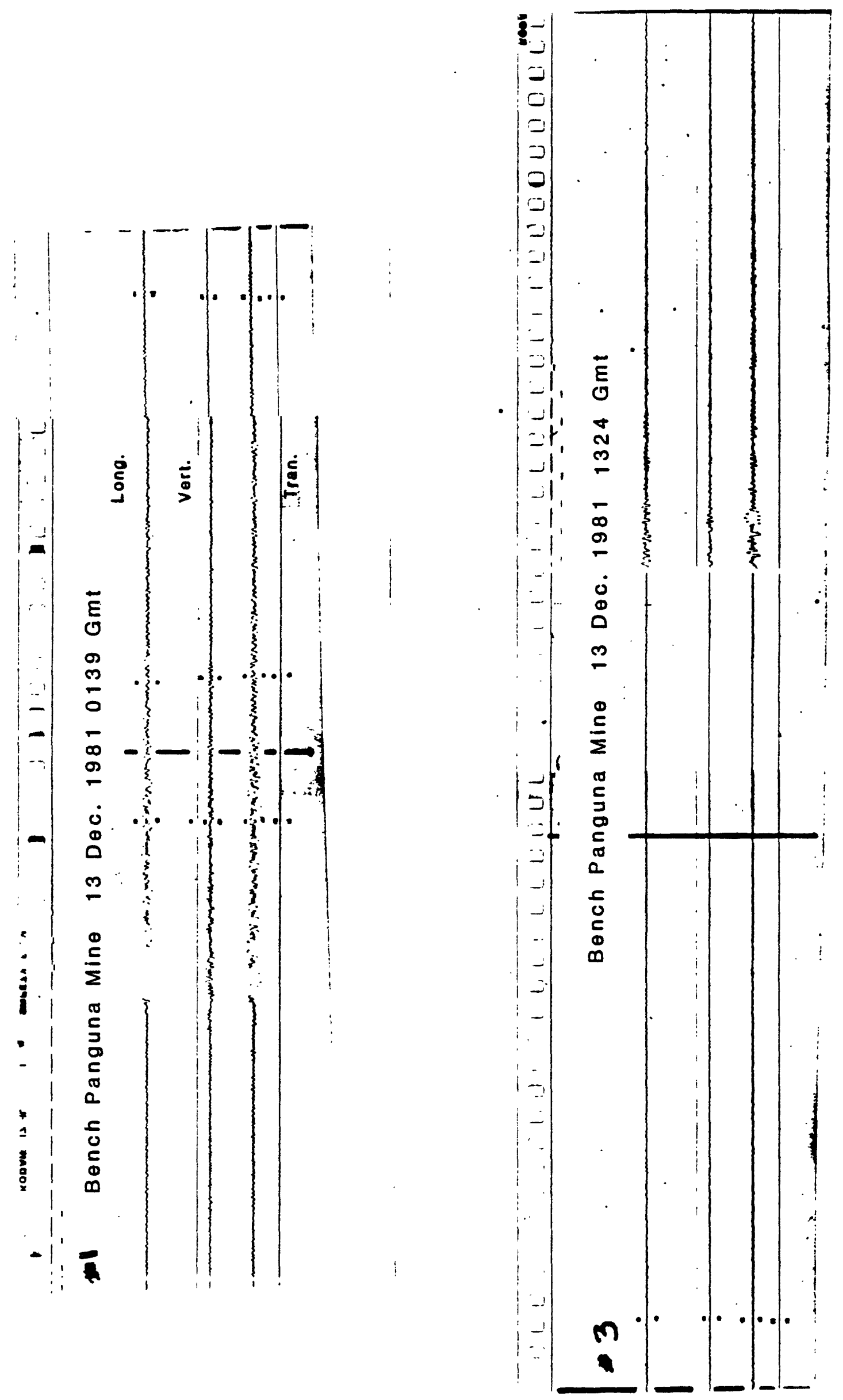

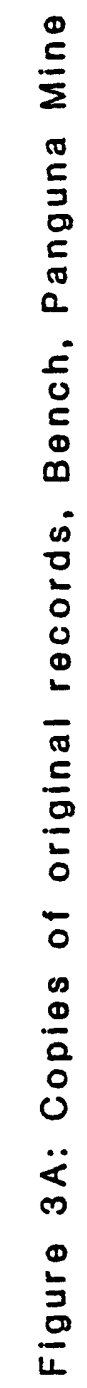




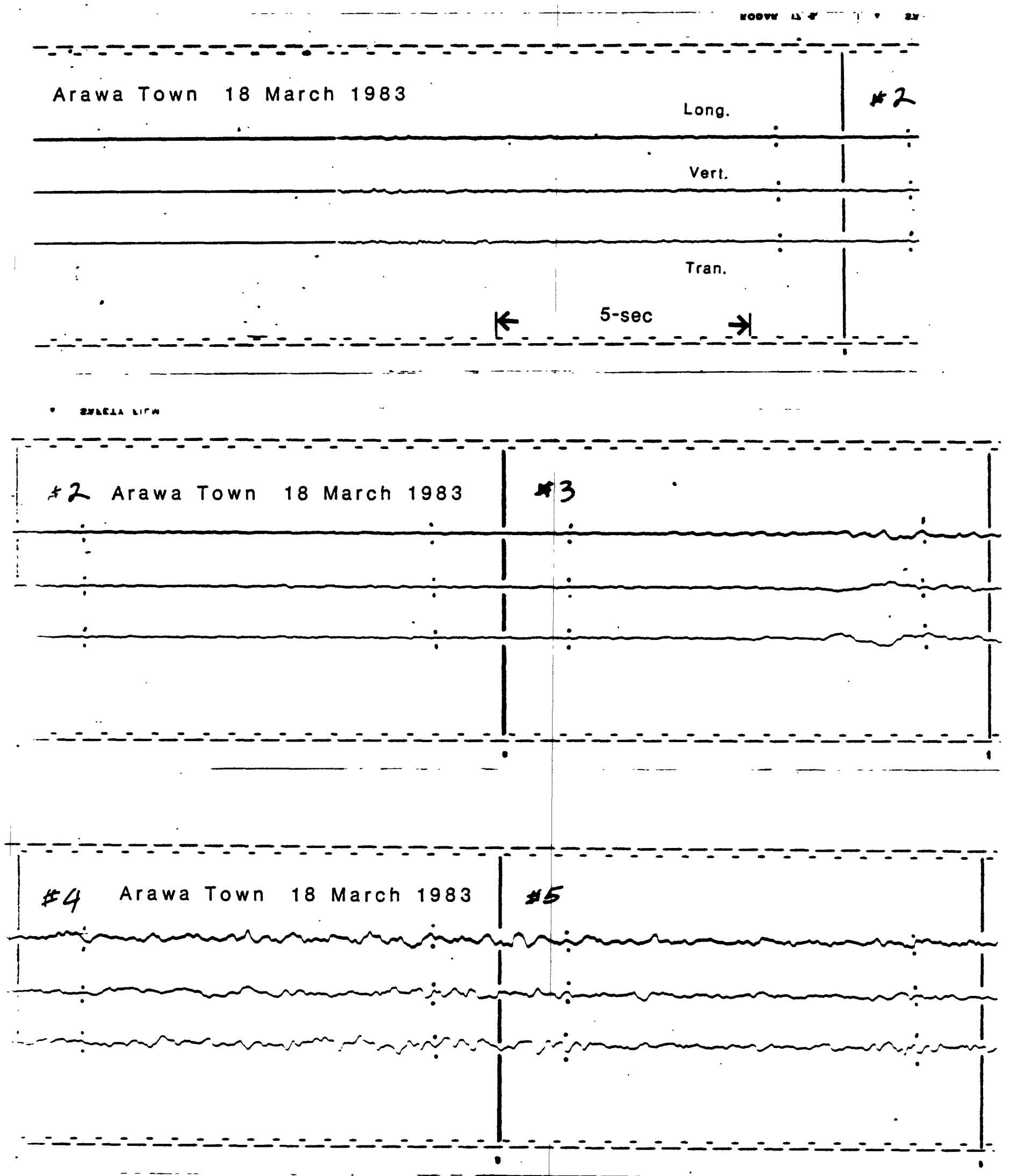

Figure 3B: Copies of original records, Arawa Town 

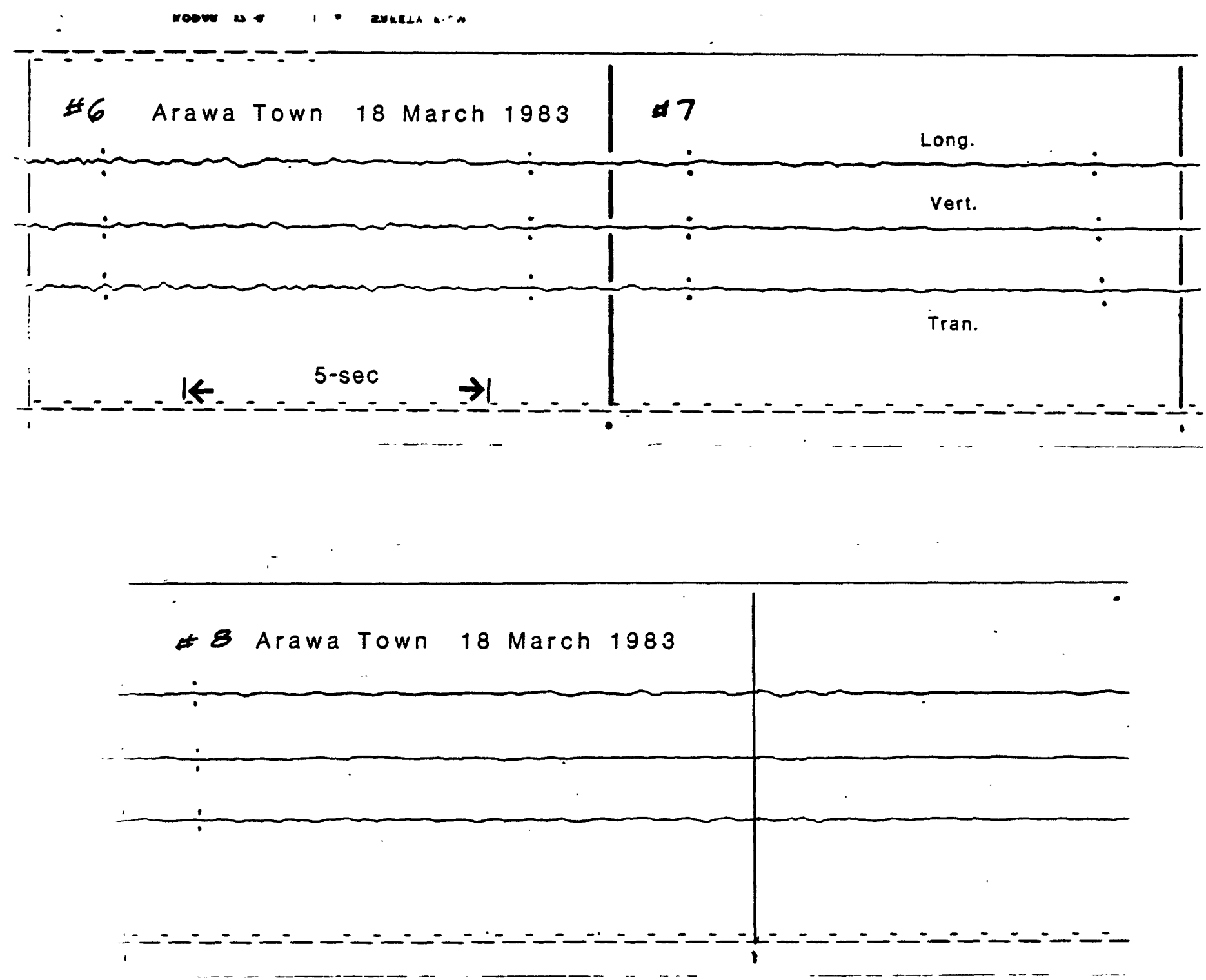

Figure 3B: continued 

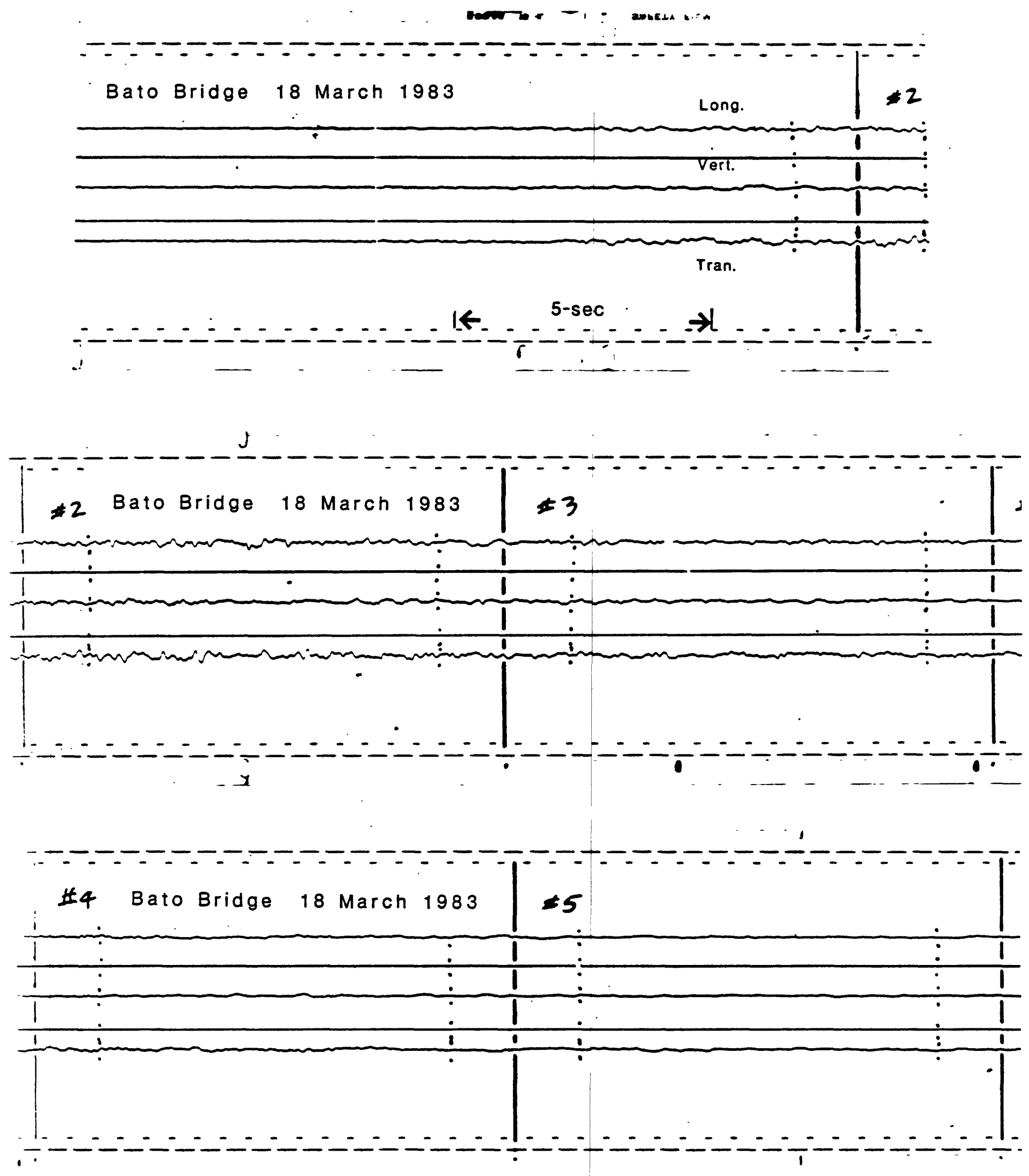

Figure 3C: Copies of original records, Bato Bridge. 

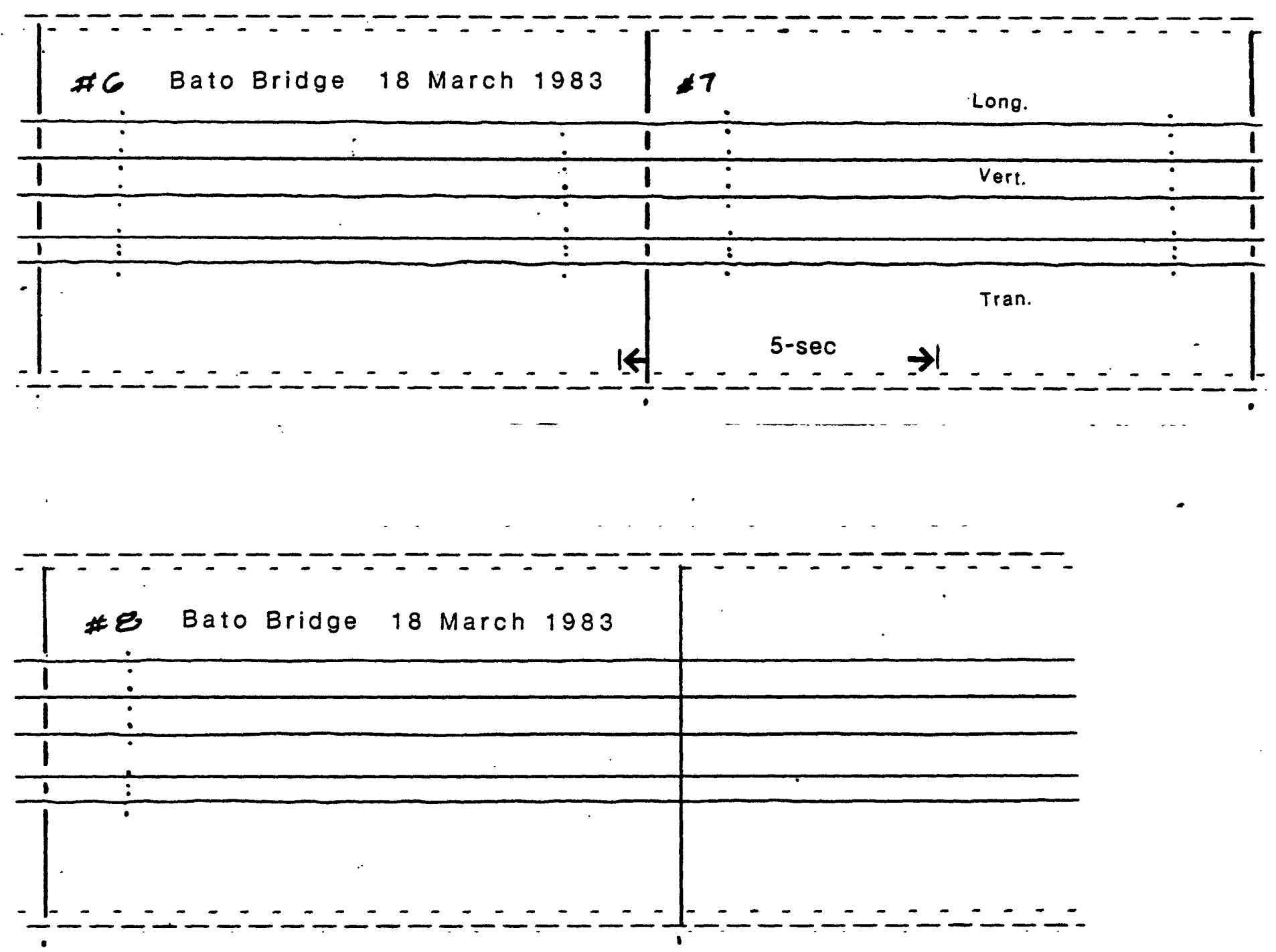

Figure $3 C$ : continued 


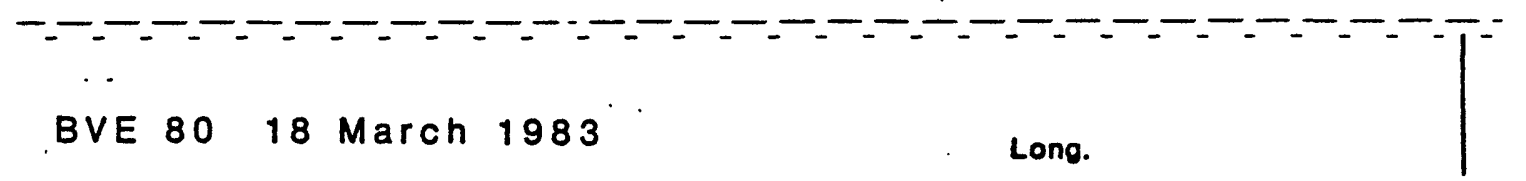

BVE 8018 March 1983

Long.
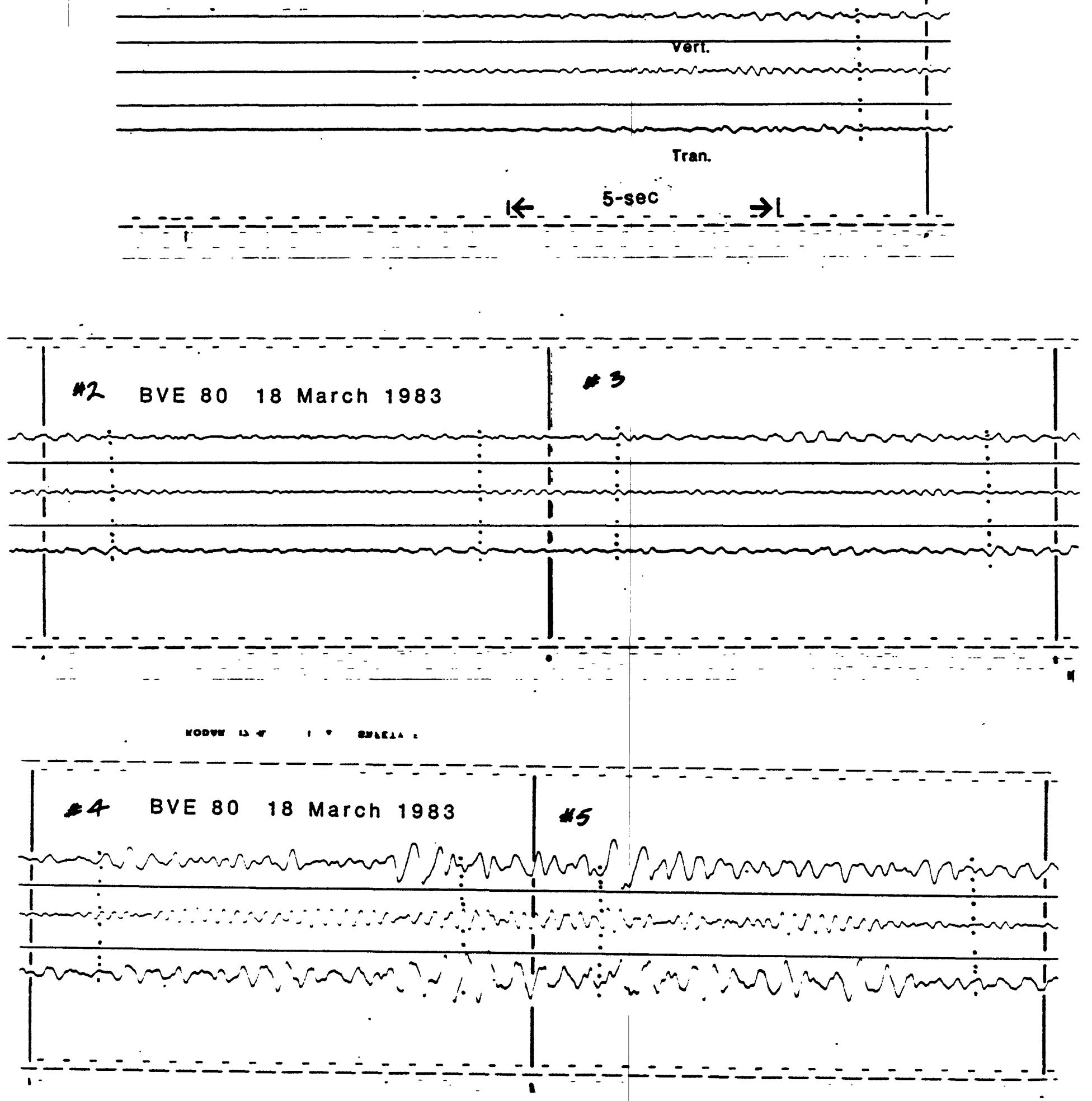

Figure 3D: Copies of original records, BVE 80 . 

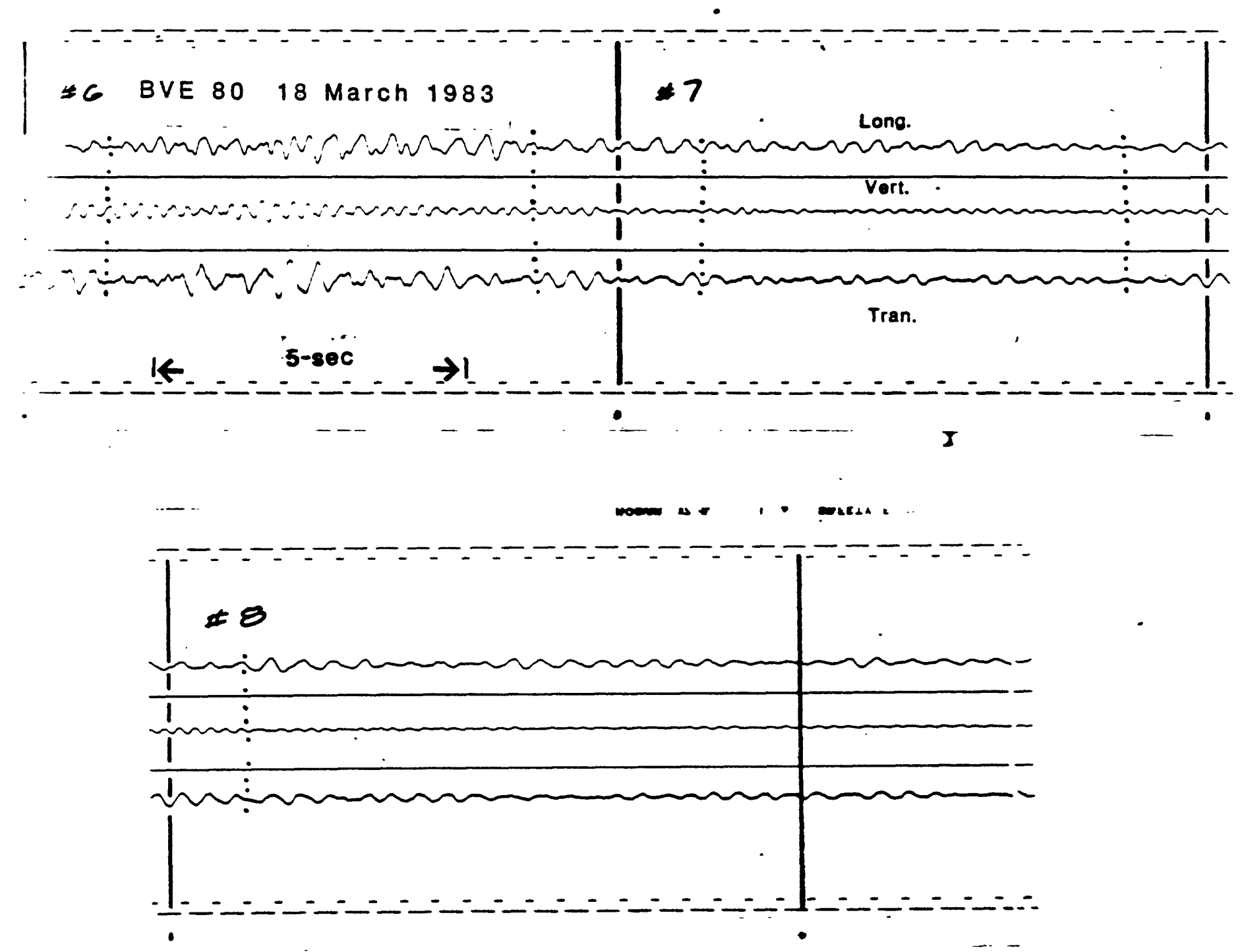

Figure 3D: continued 


\section{Appendix I}

\section{Computer Plots}




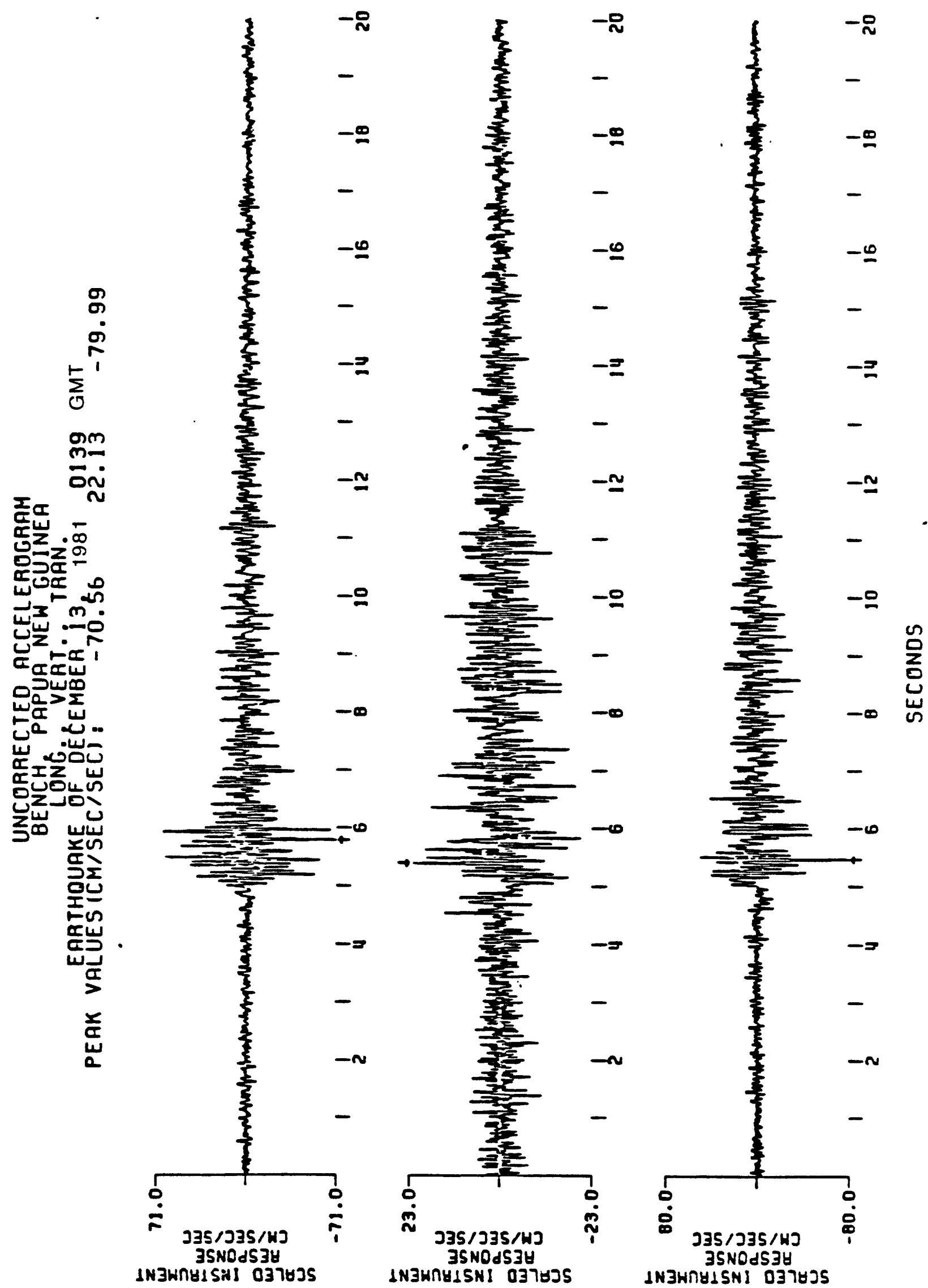




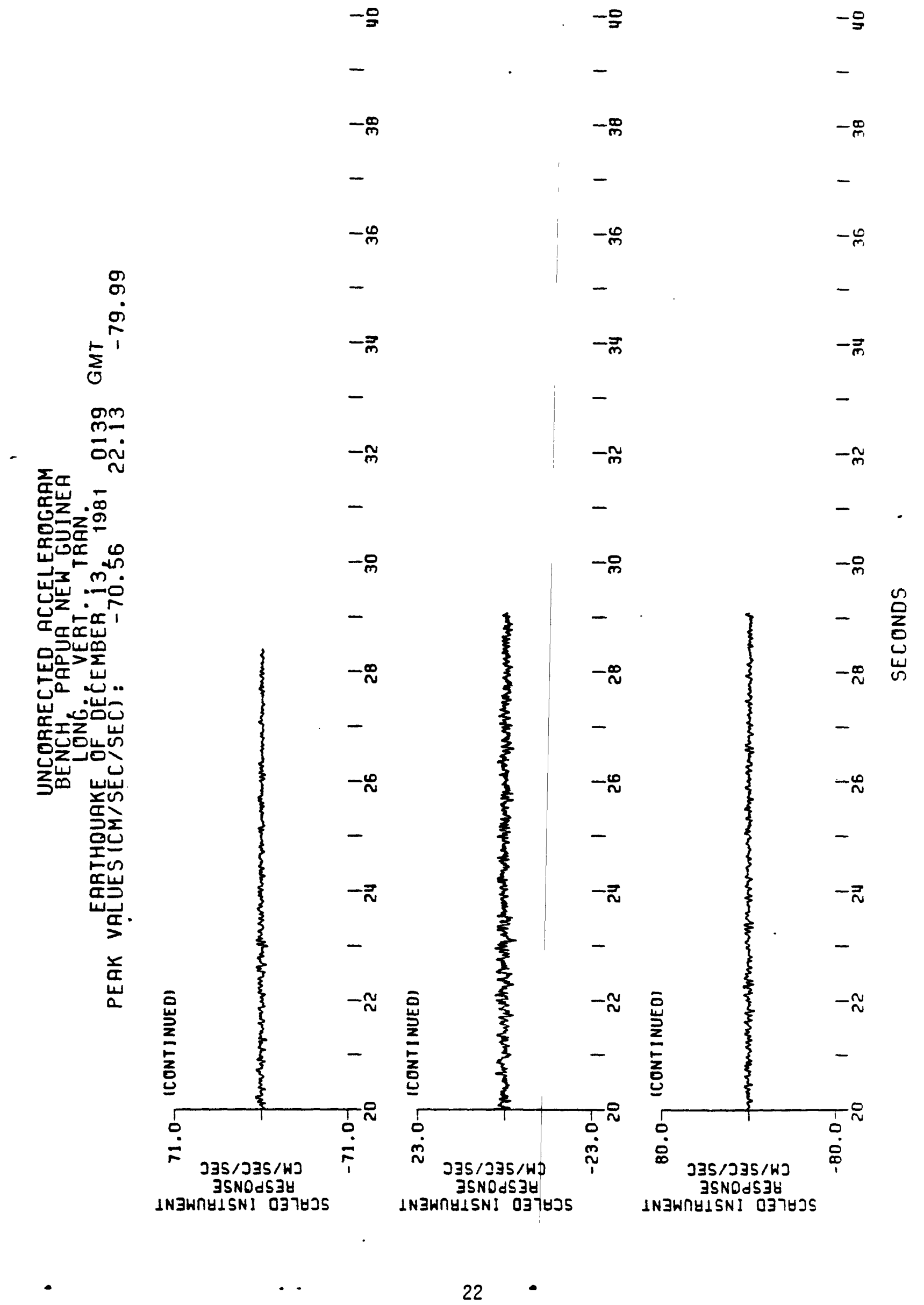




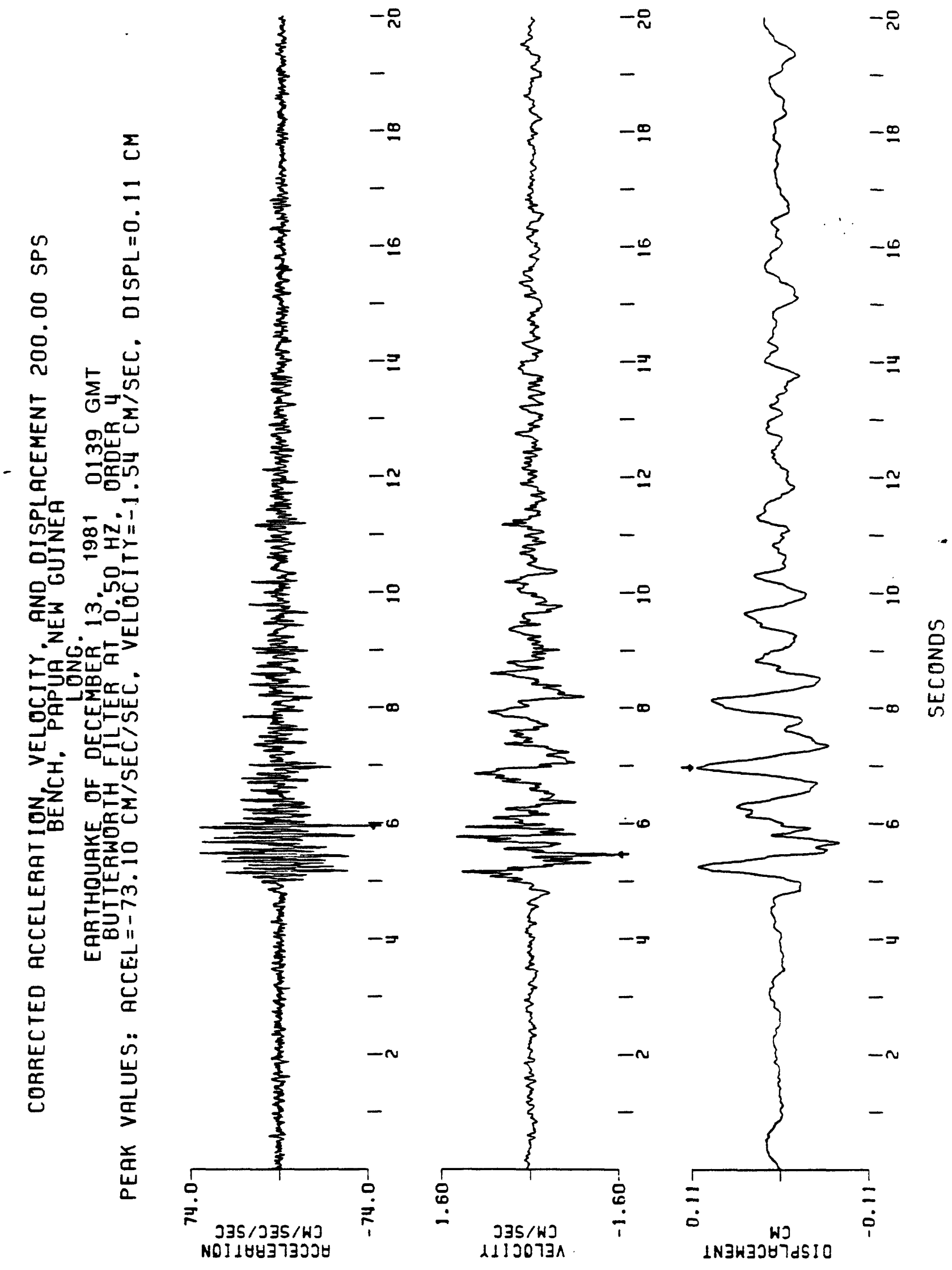




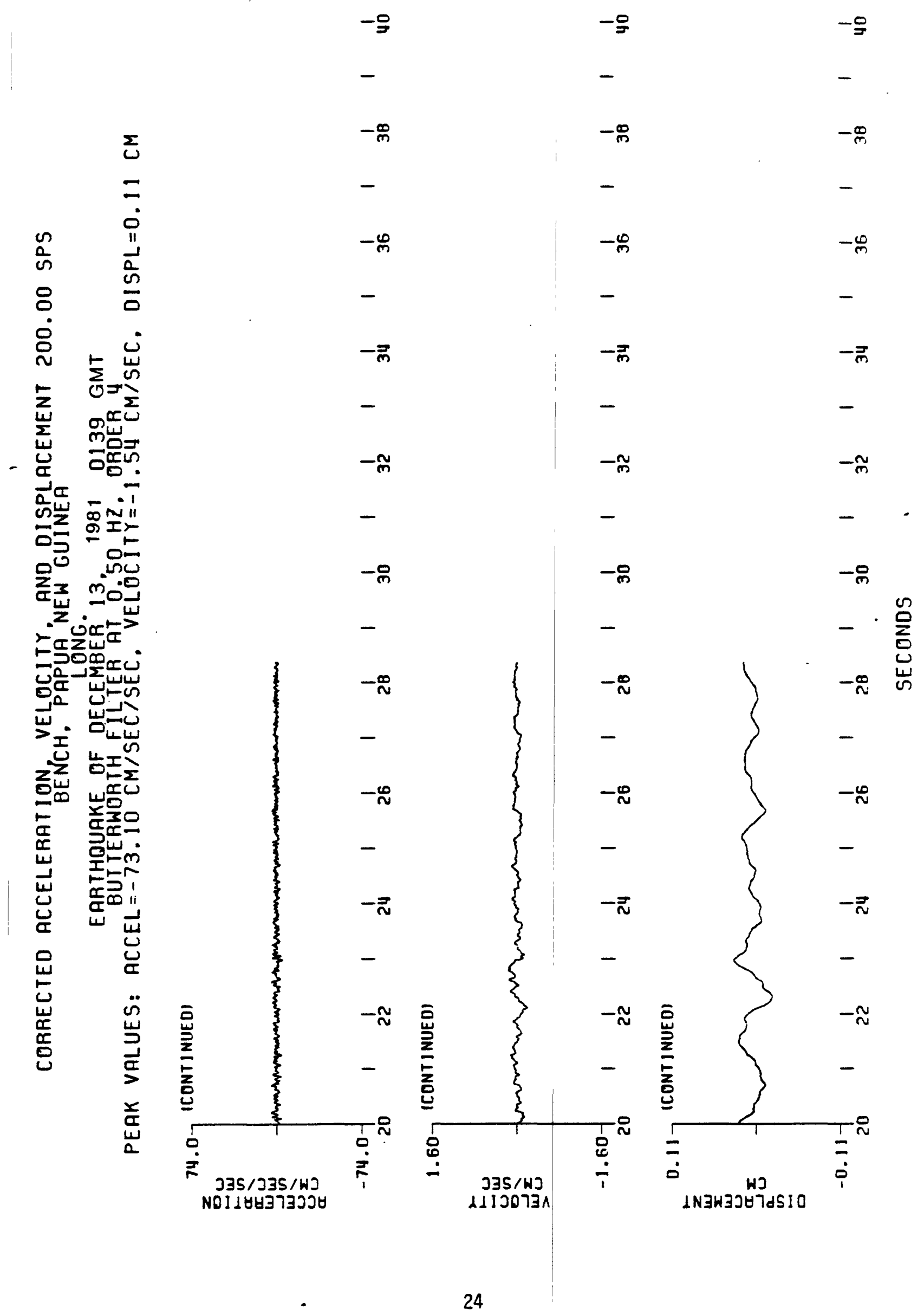




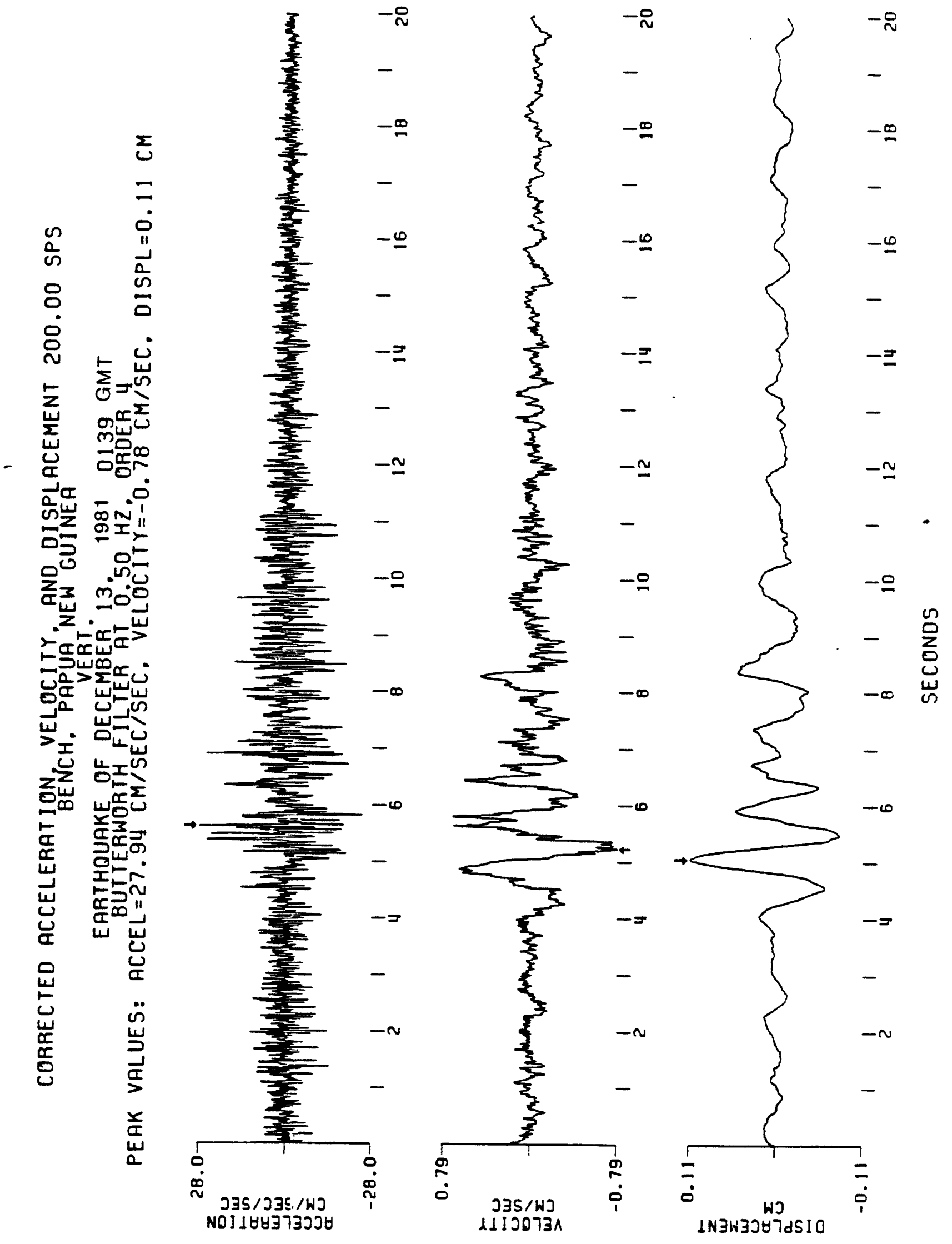


$-9$

$\sum$

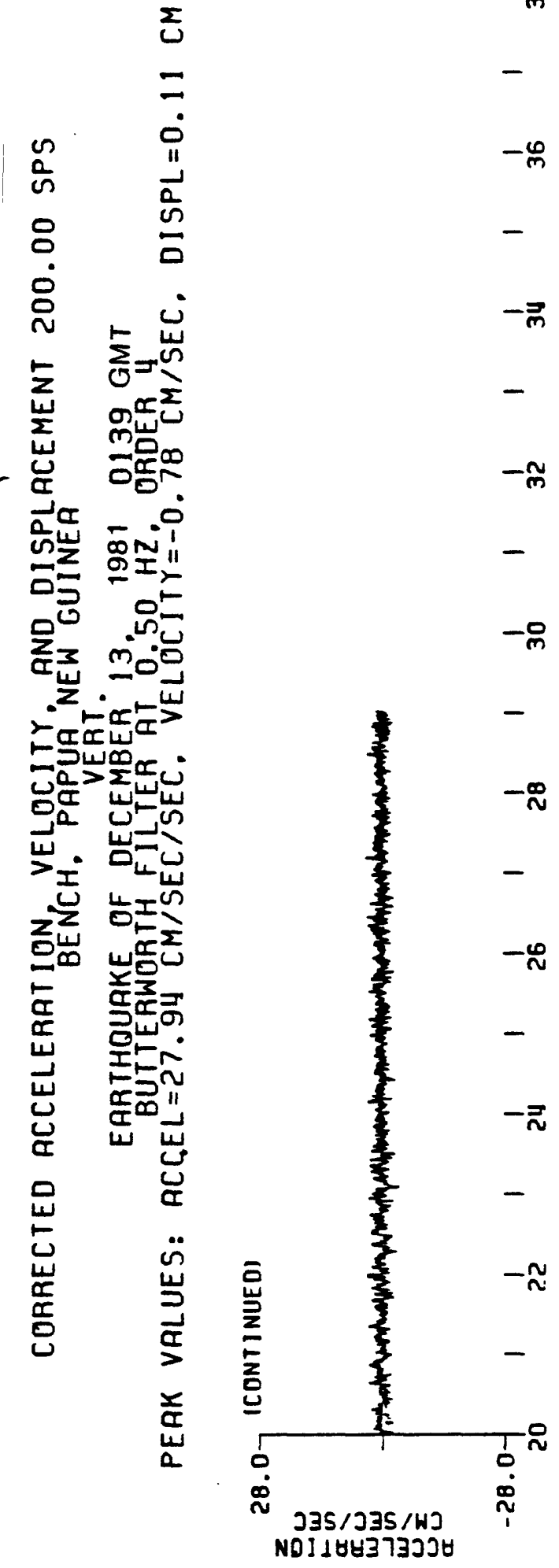

-

$-\mathscr{e}$

F

กั

$\stackrel{\infty}{\sim}$

$-\infty$

$\approx$
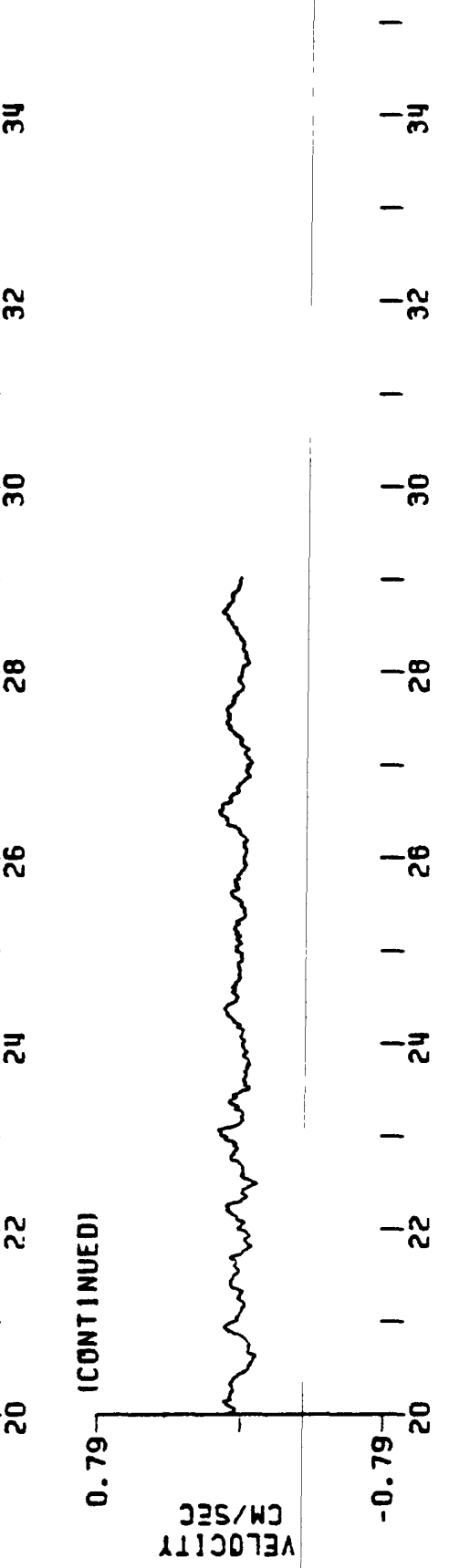

-

$-$

$-\approx$

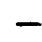

$-8$

$-\infty$

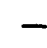

$-\infty$

$-$

$-\pi$
$-0$

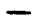

$-\infty$

$-$

$-\infty$

$-\bar{m}$

$-\tilde{m}$

$-8$

- 号

-

- चี
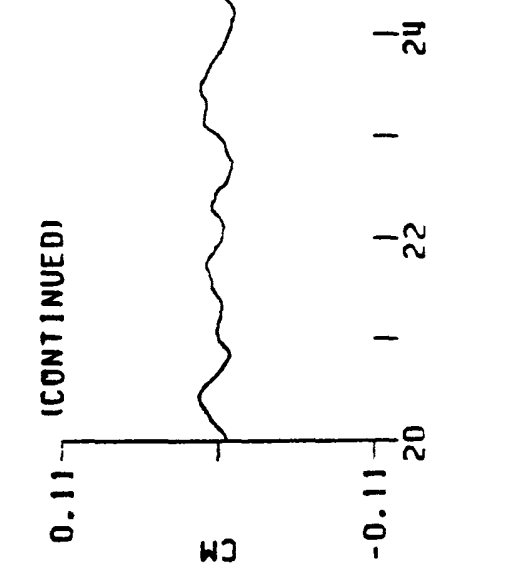

LN3H3วษาปS1O 


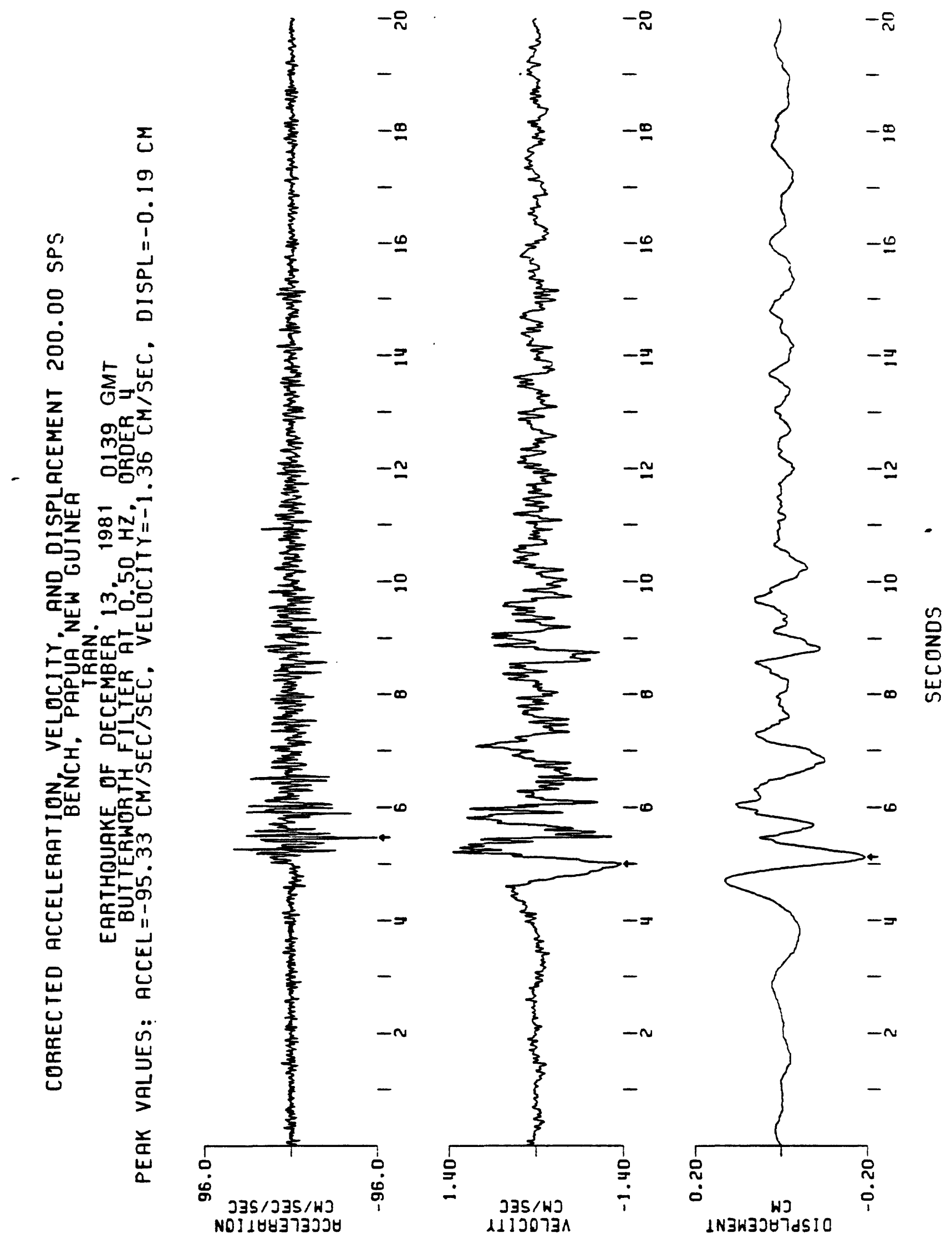




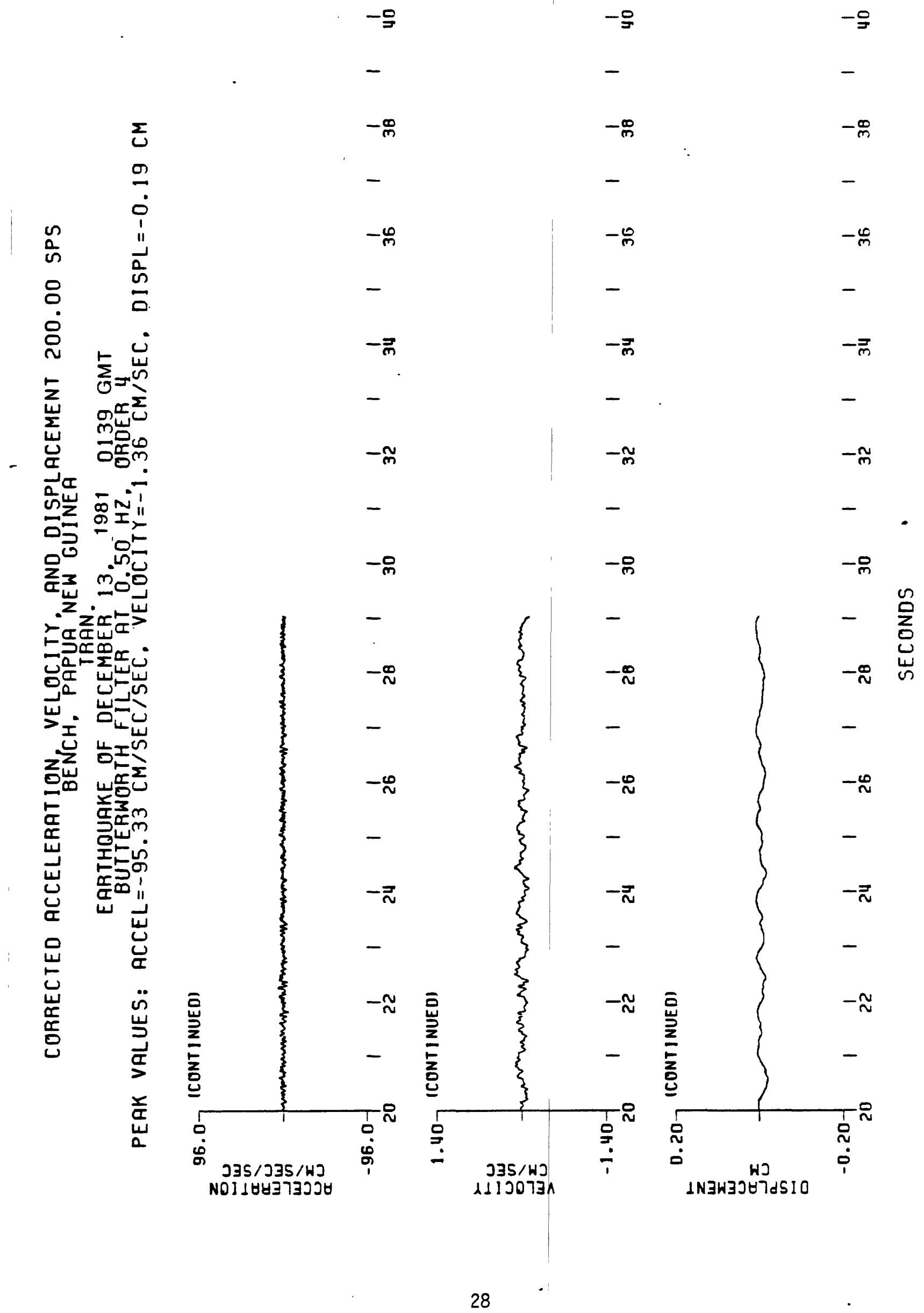




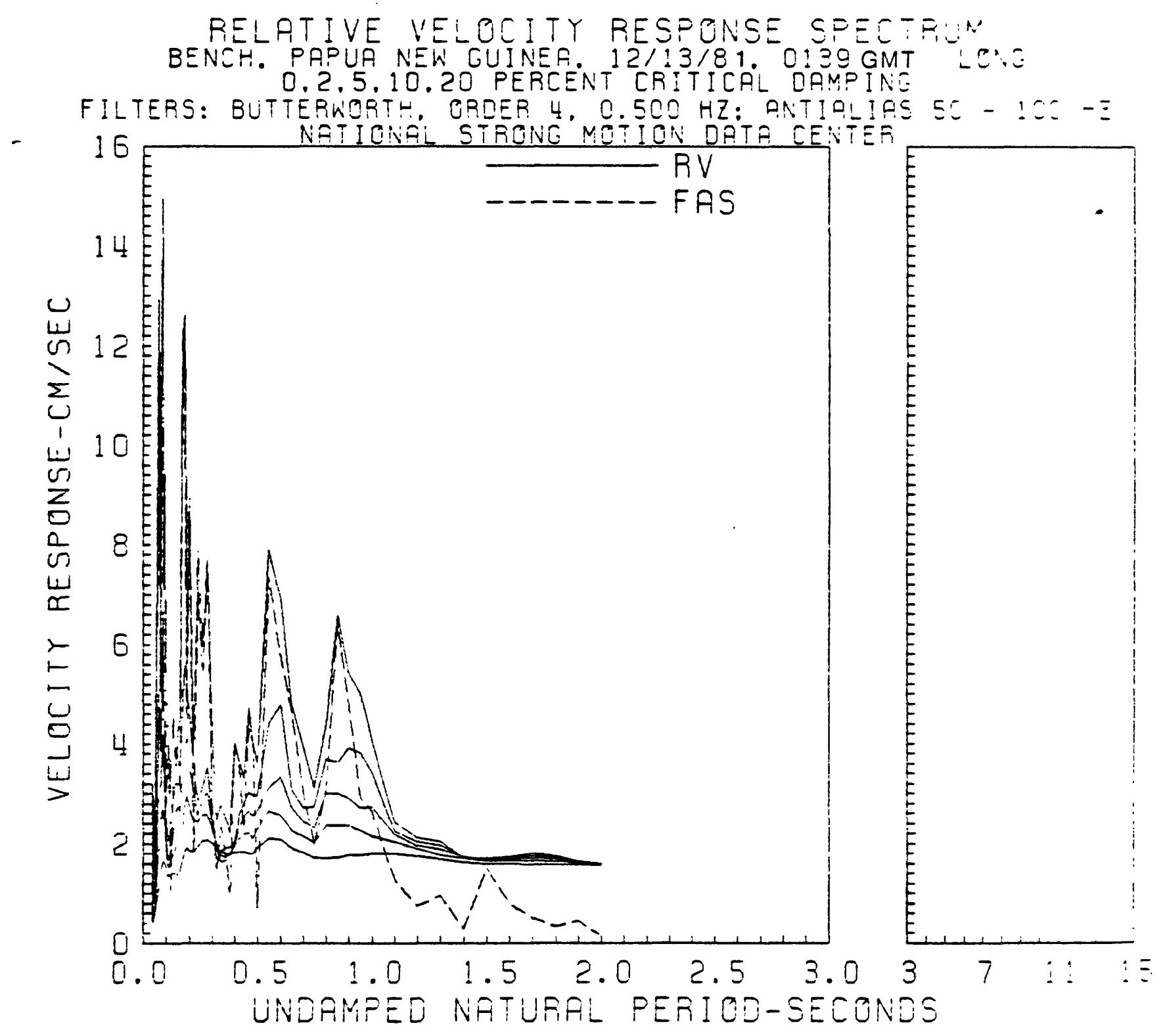




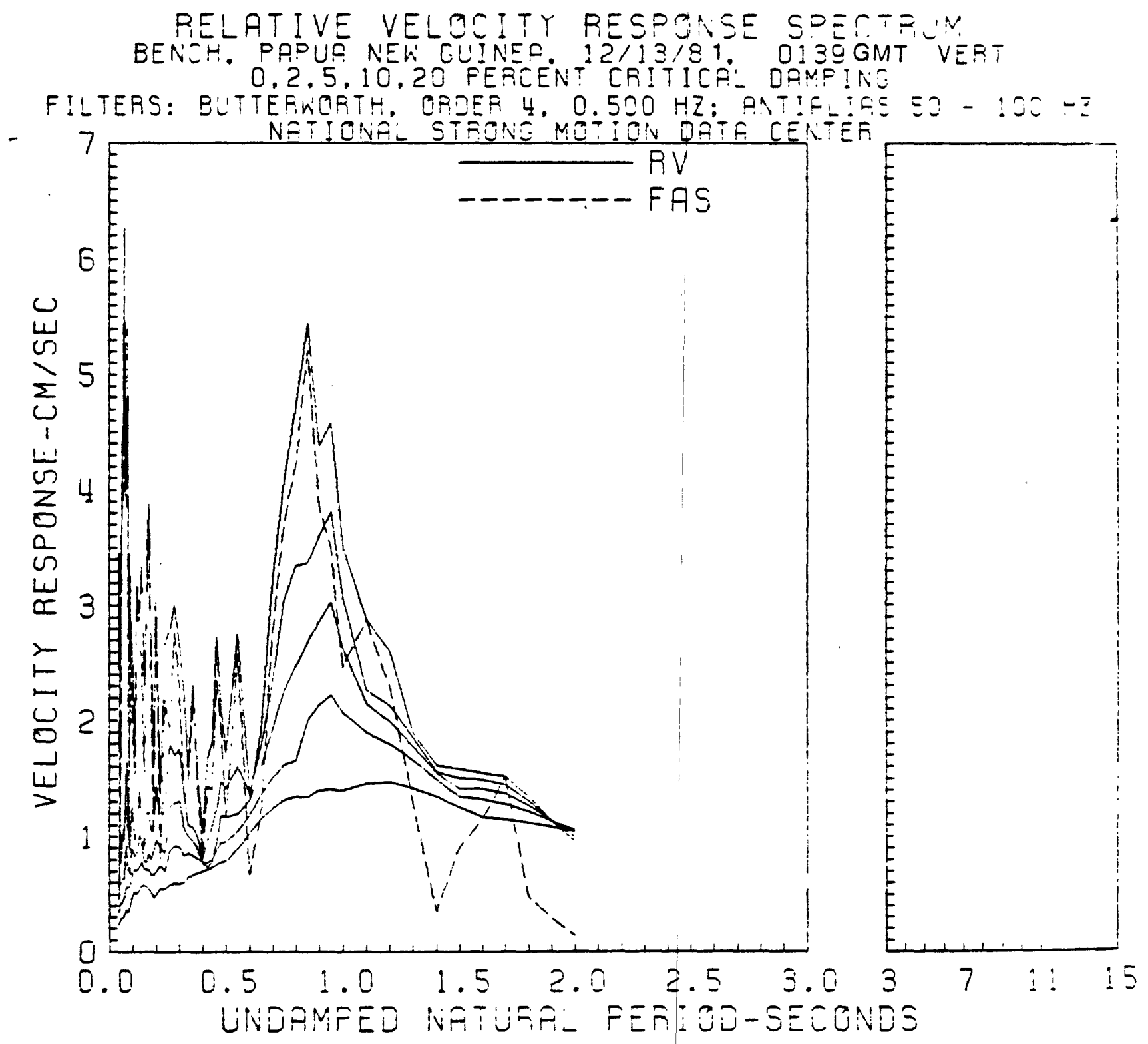




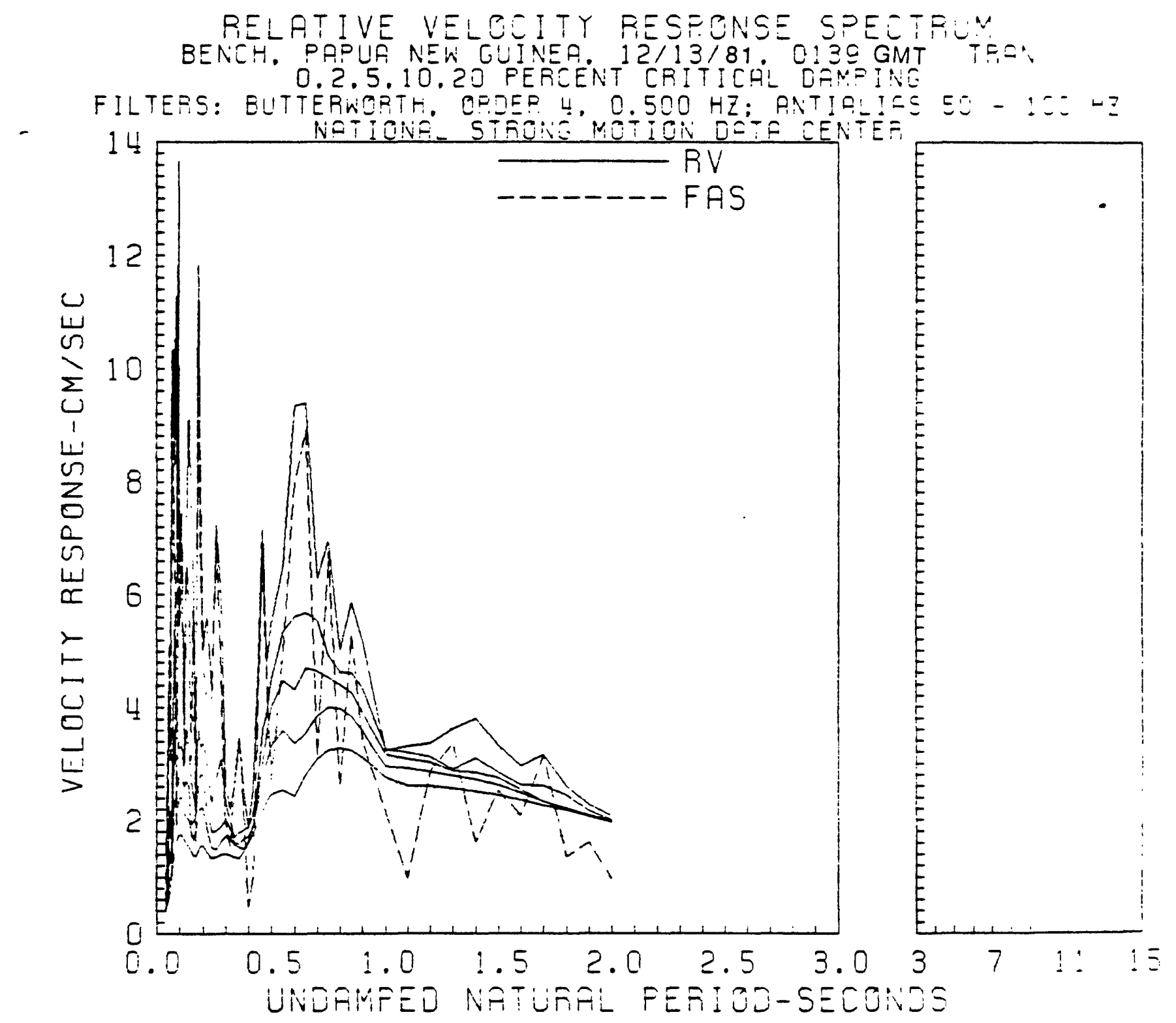




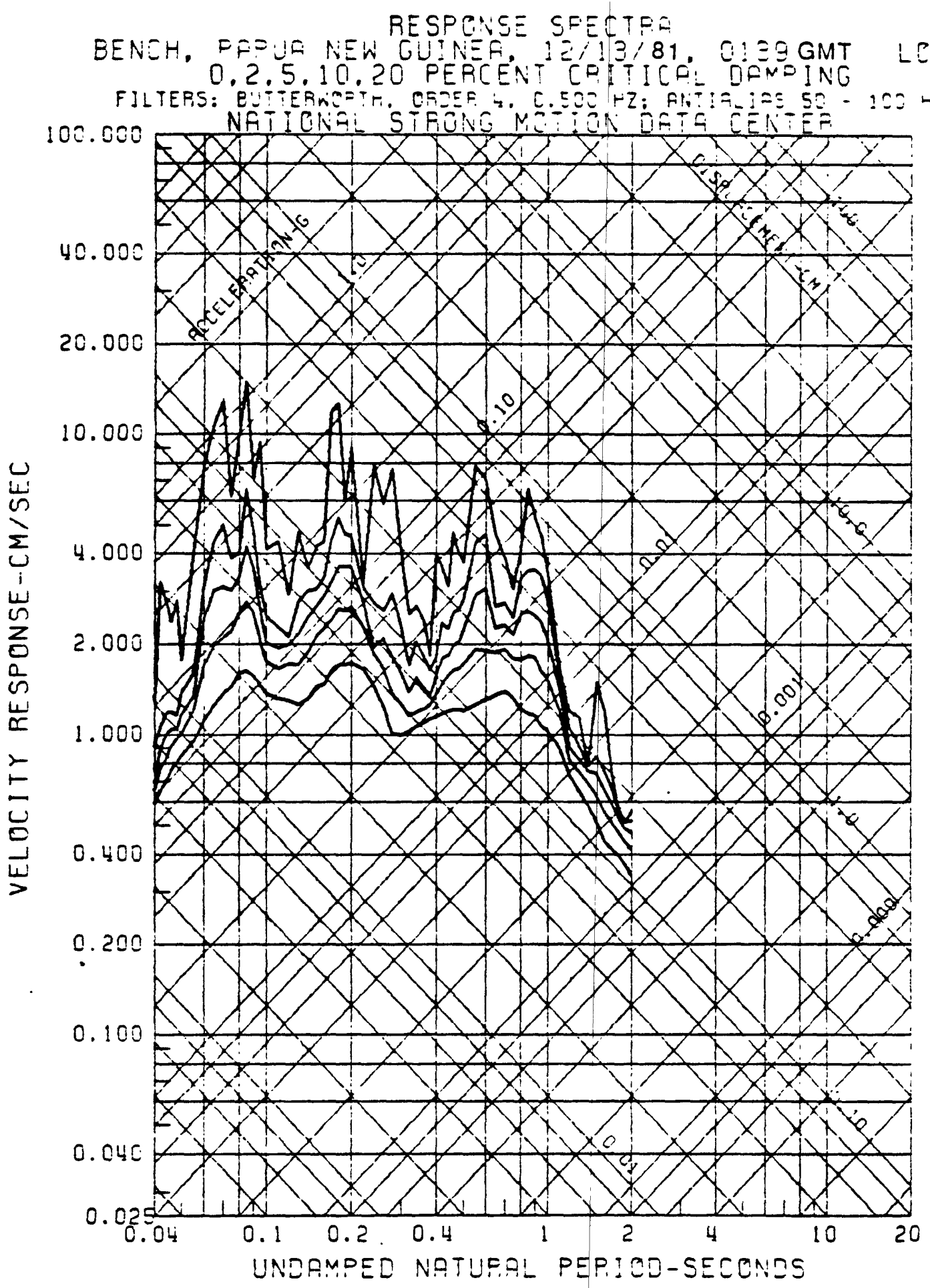




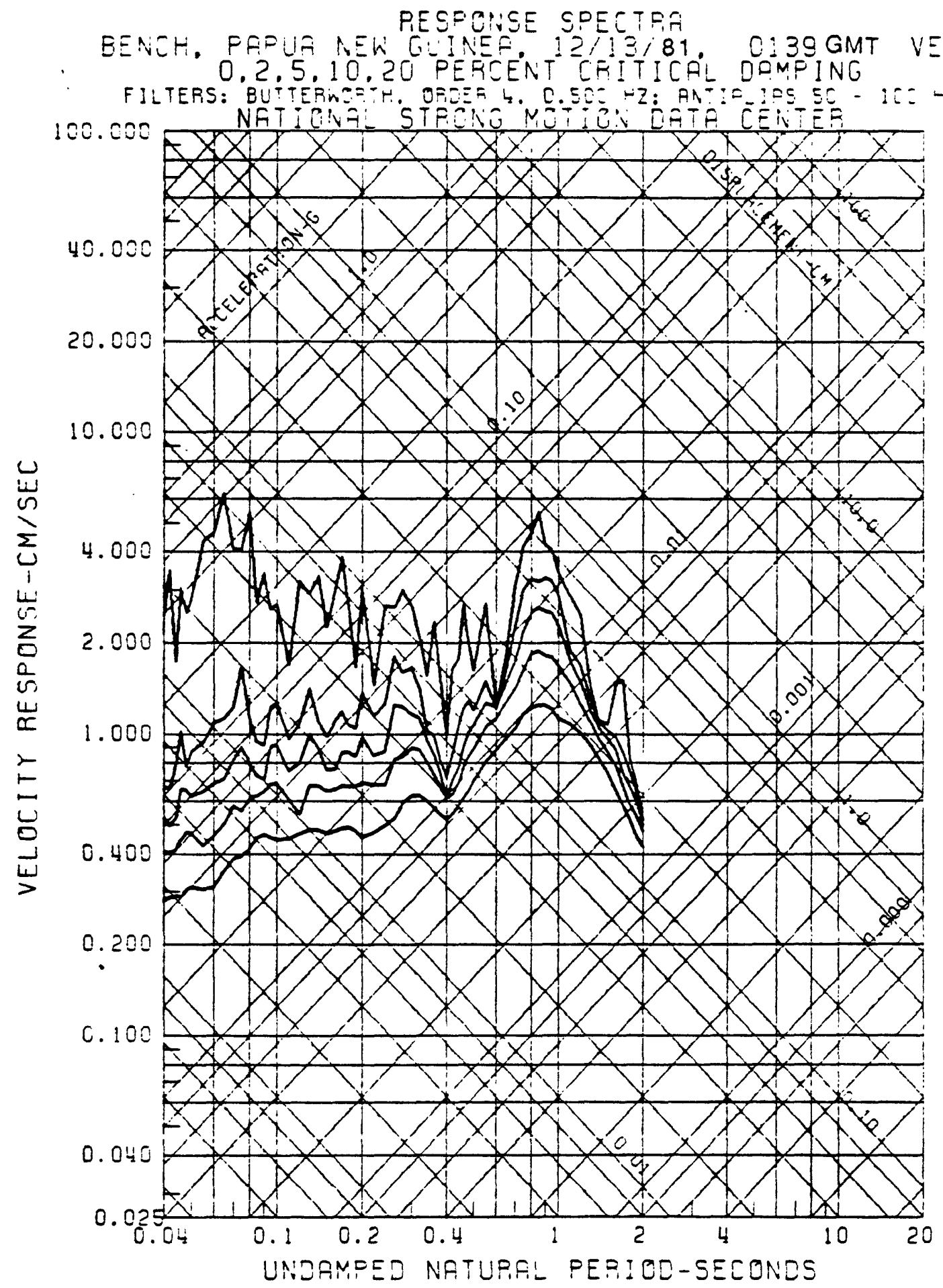




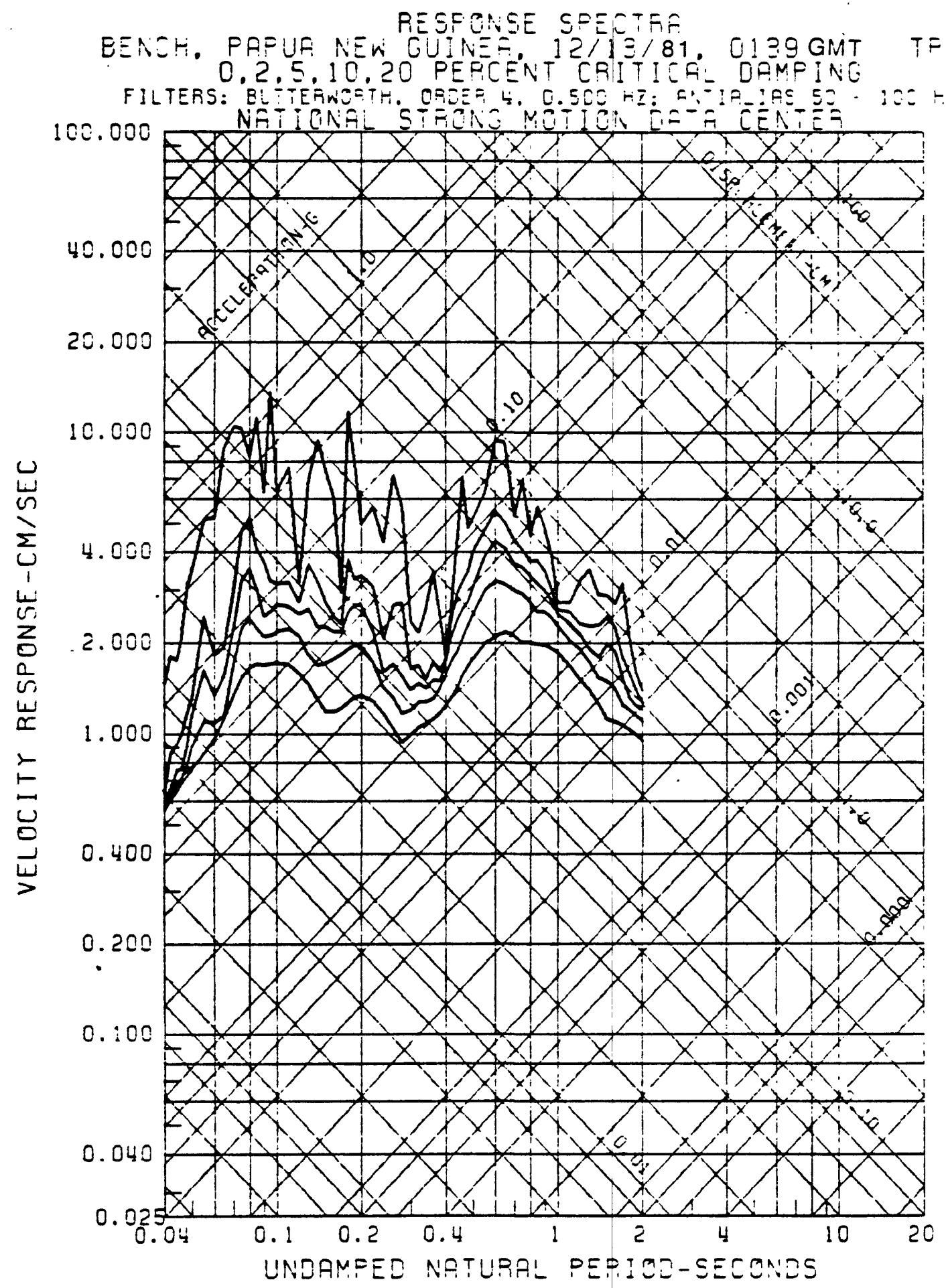




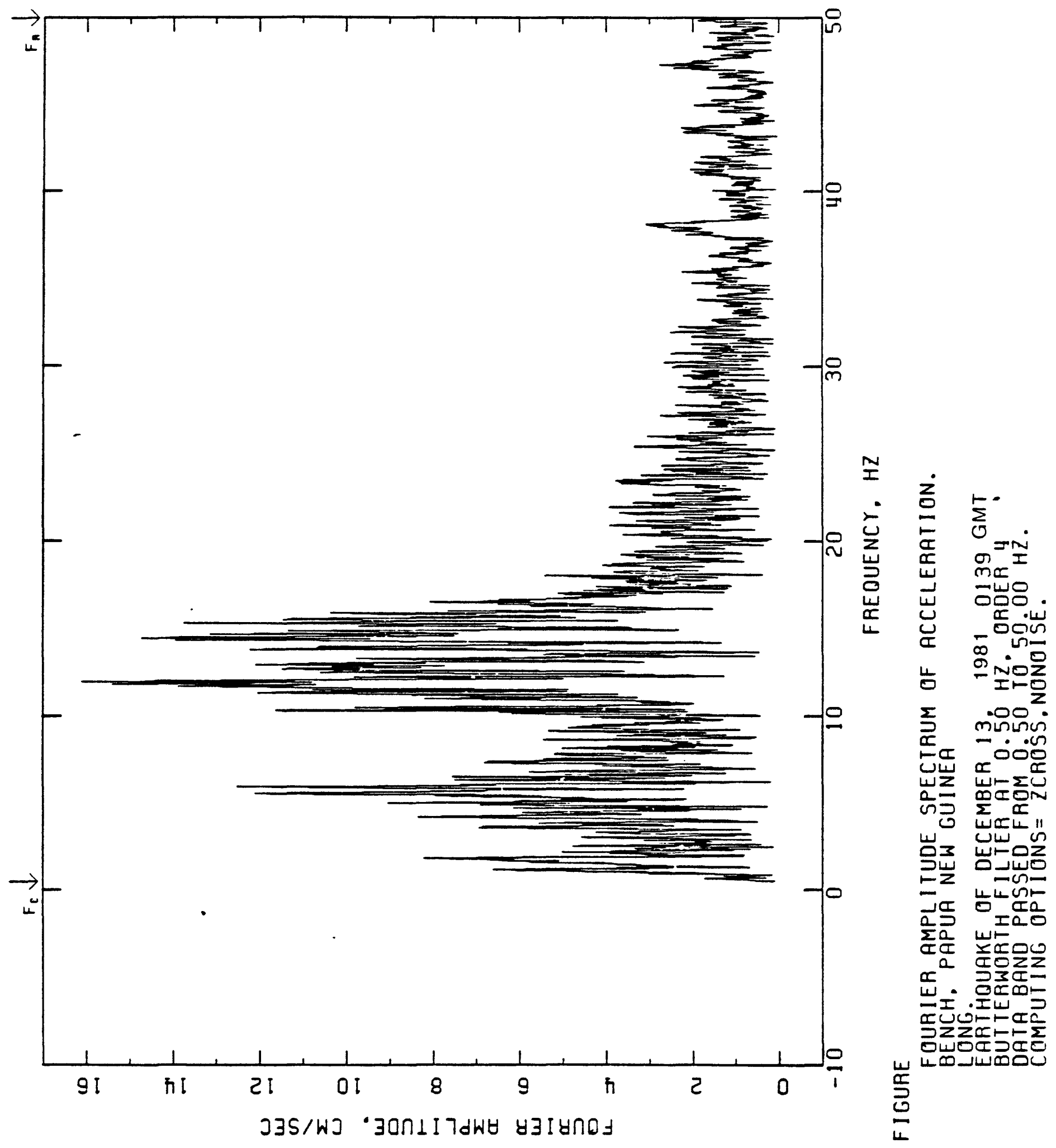




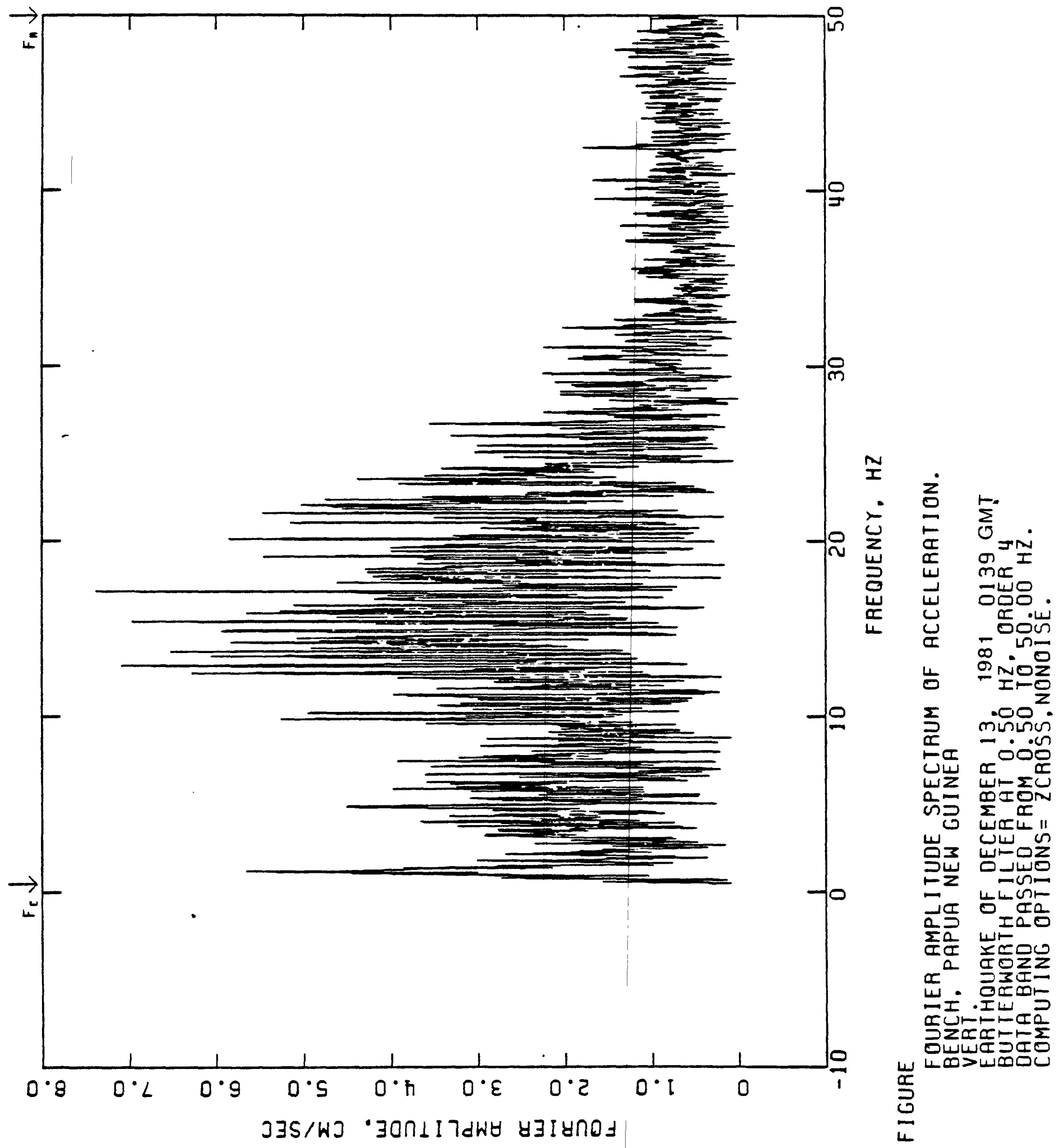




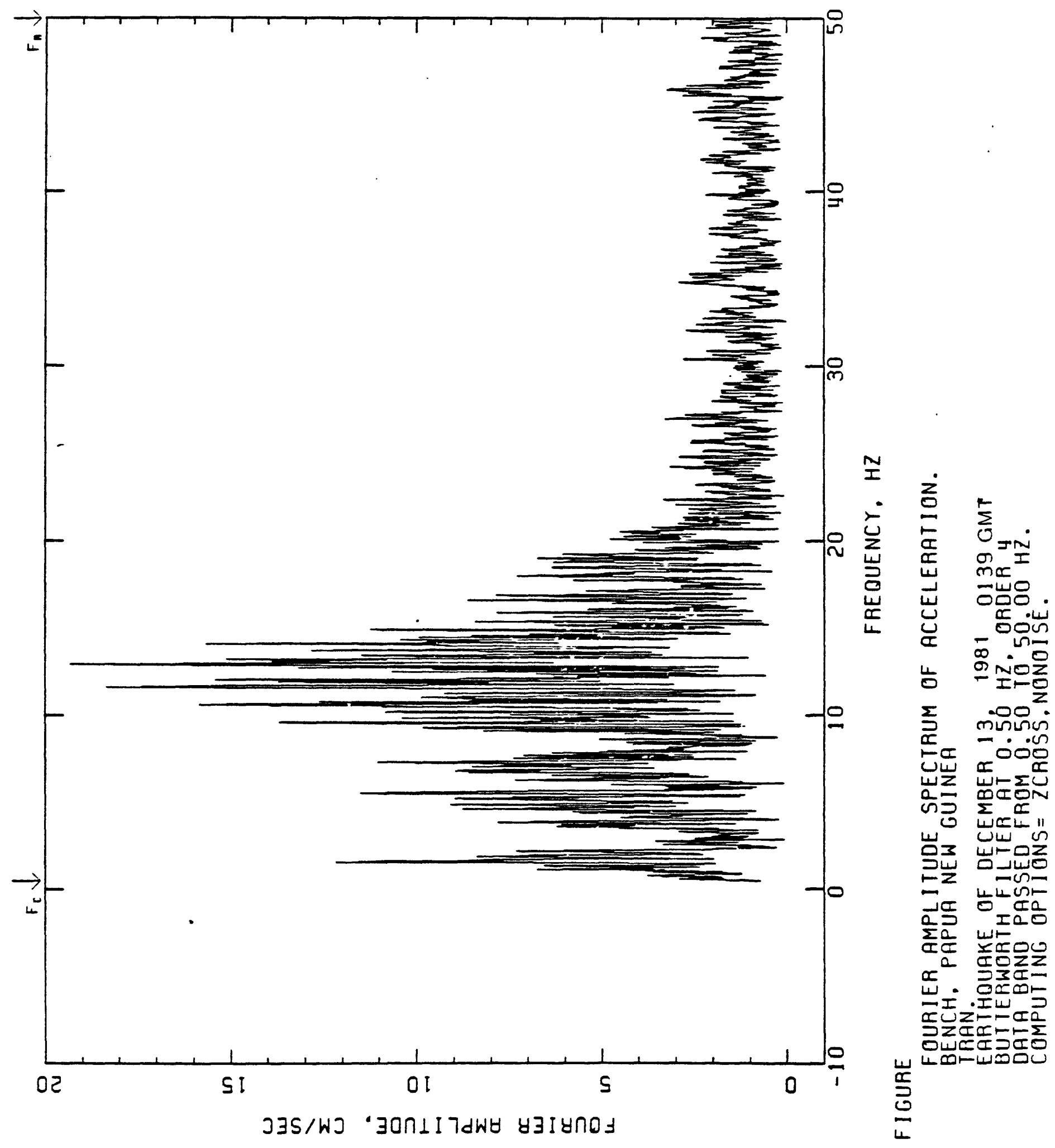




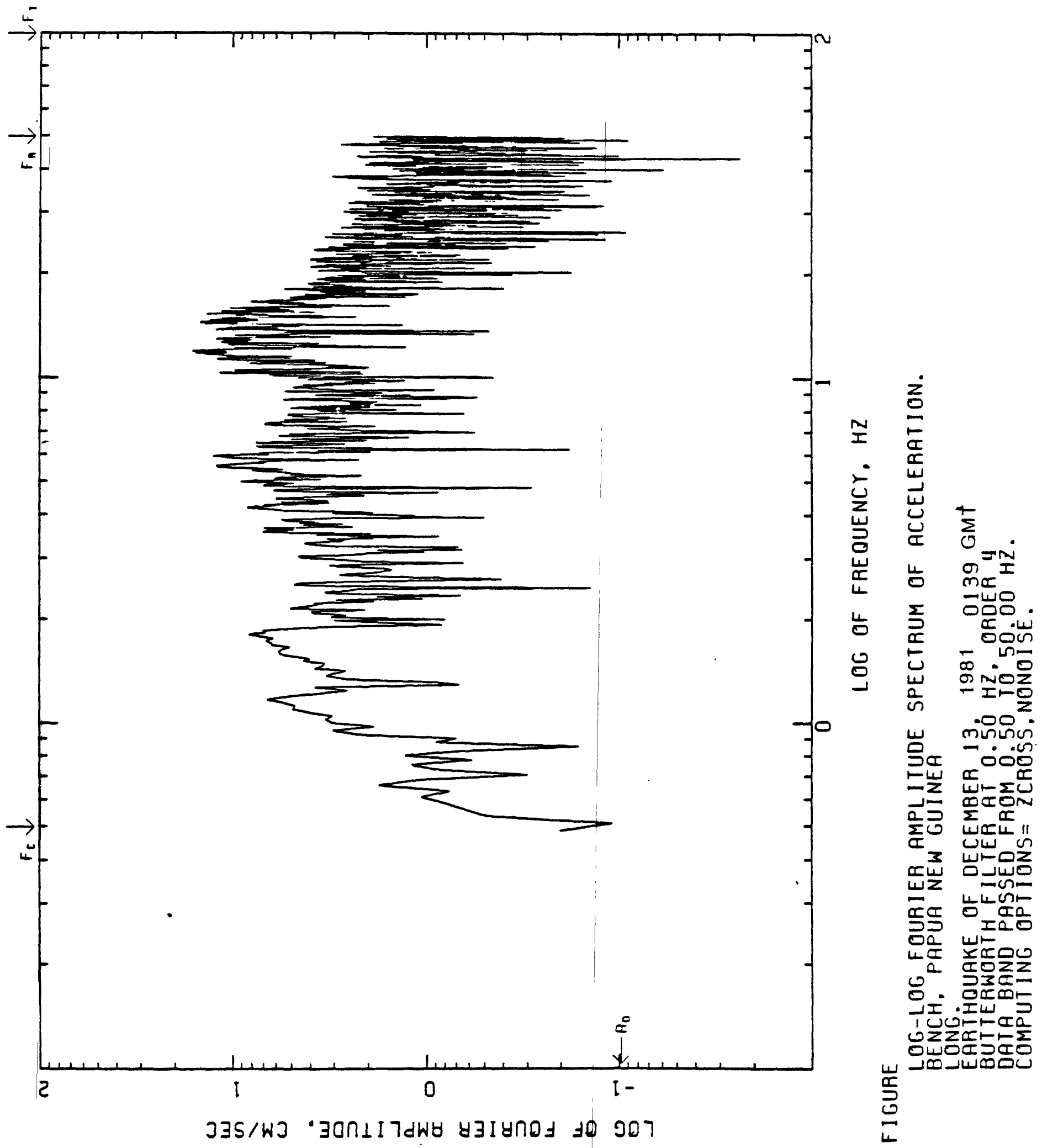




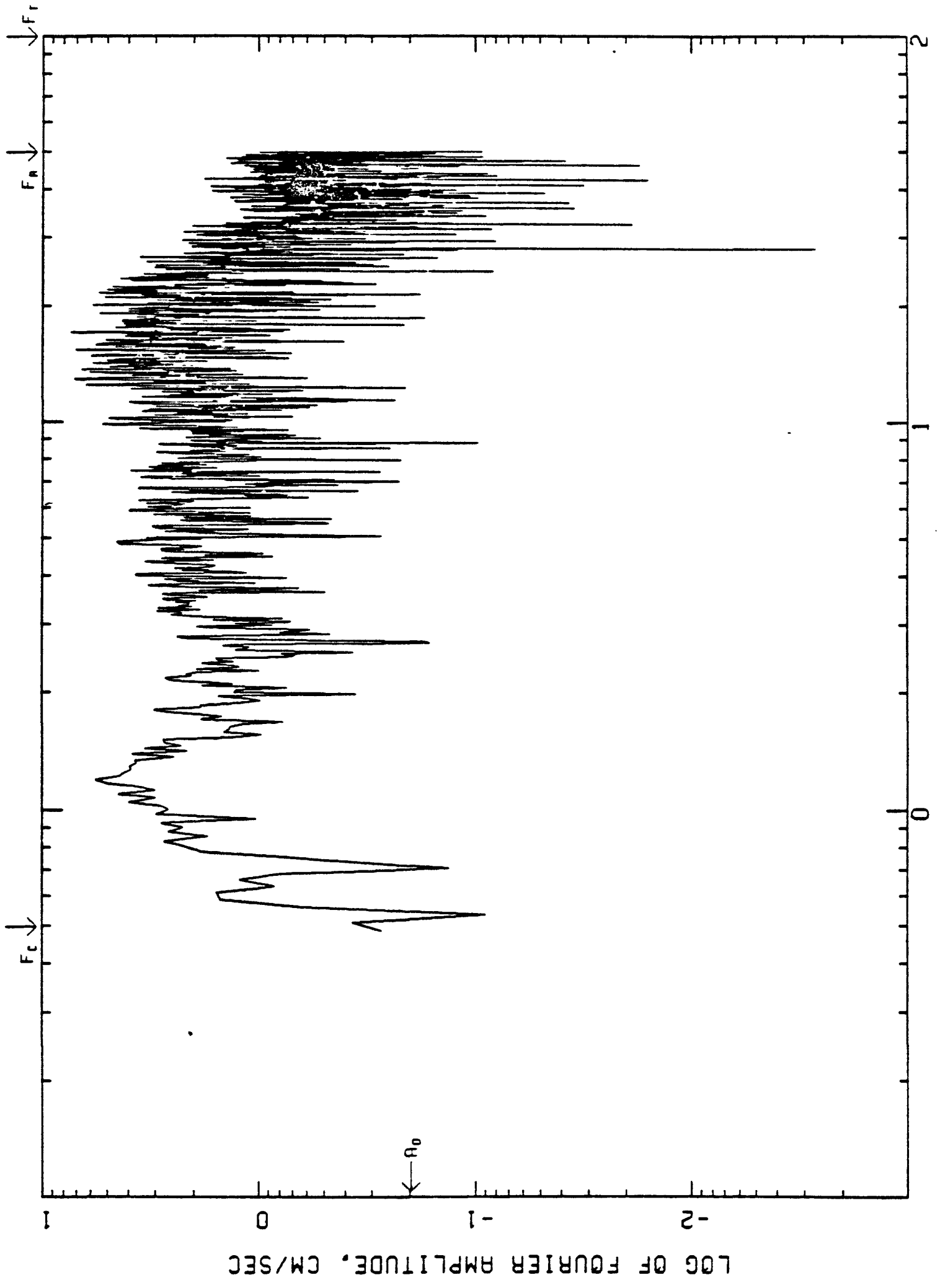

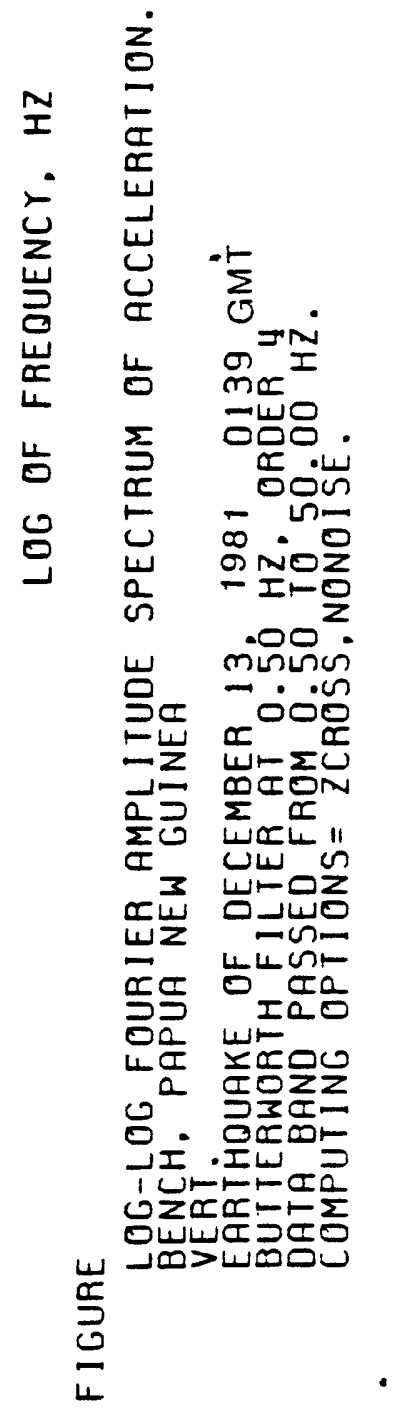




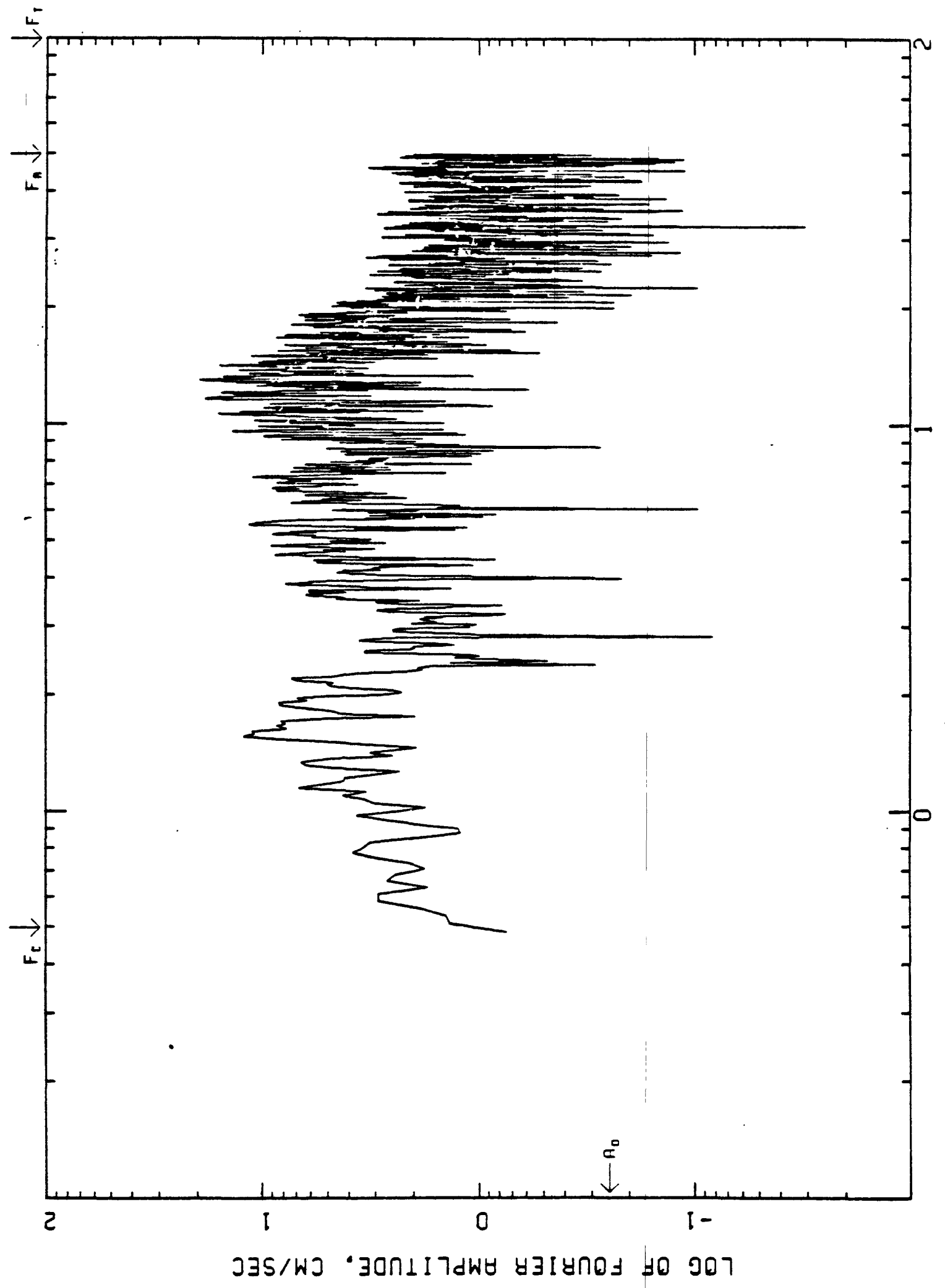

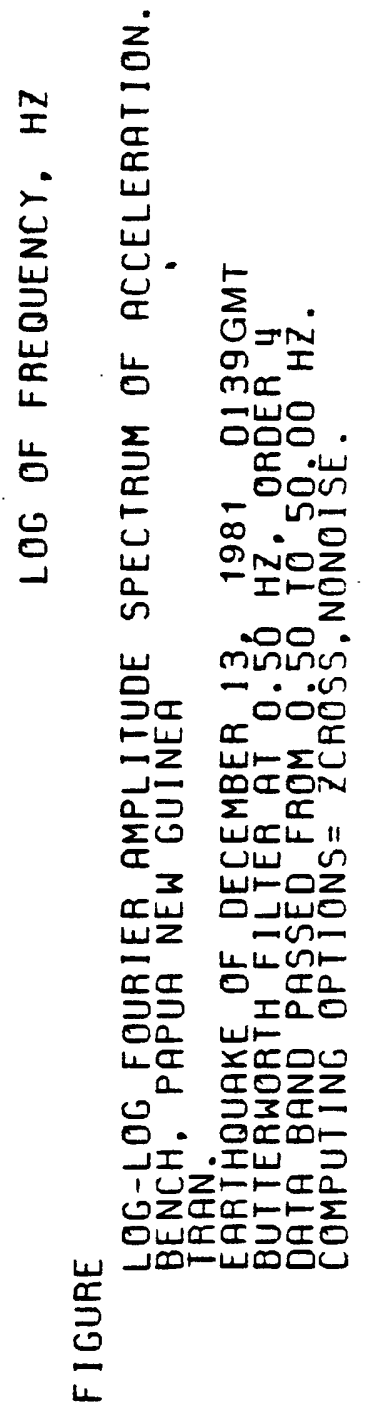




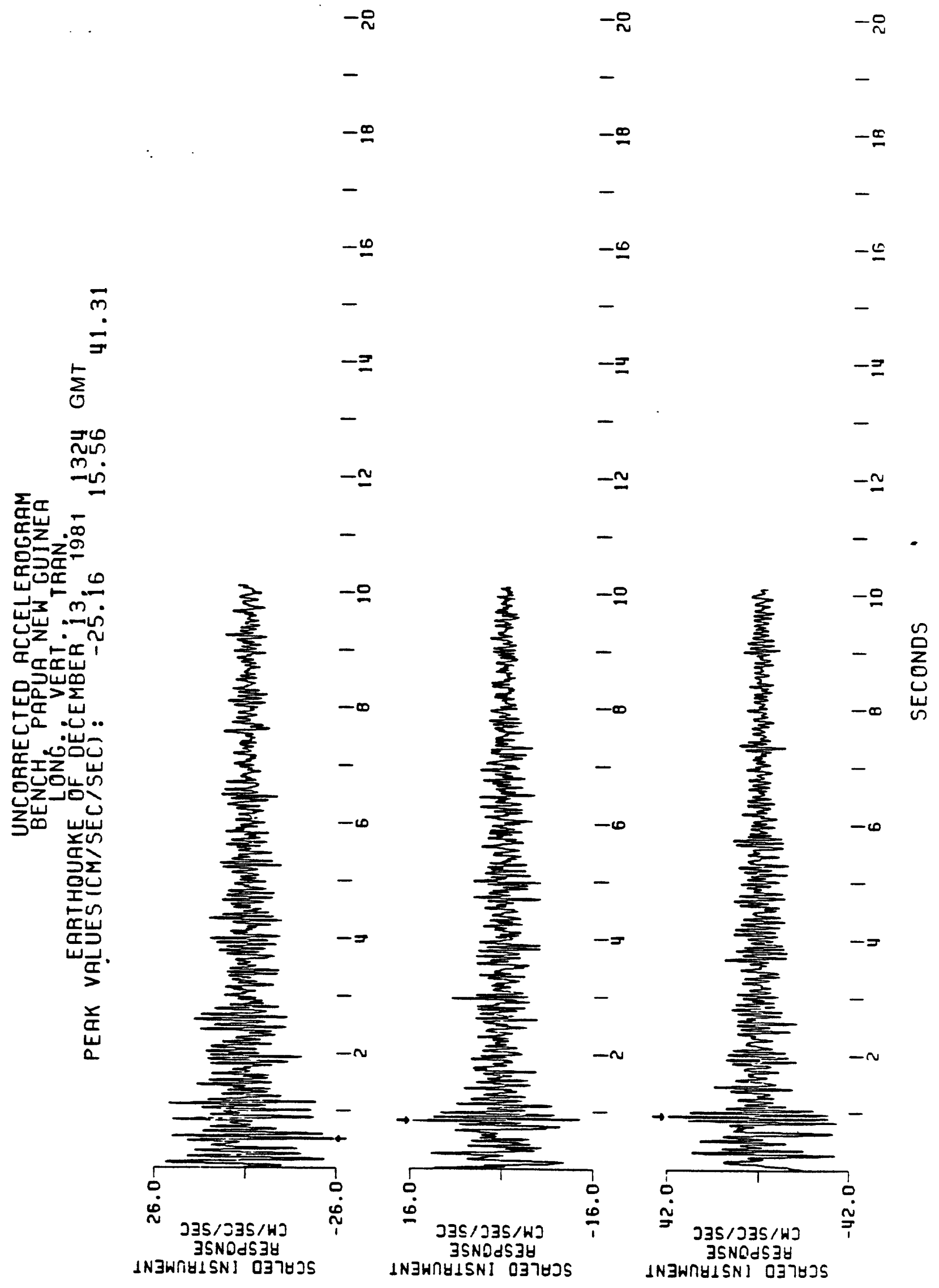




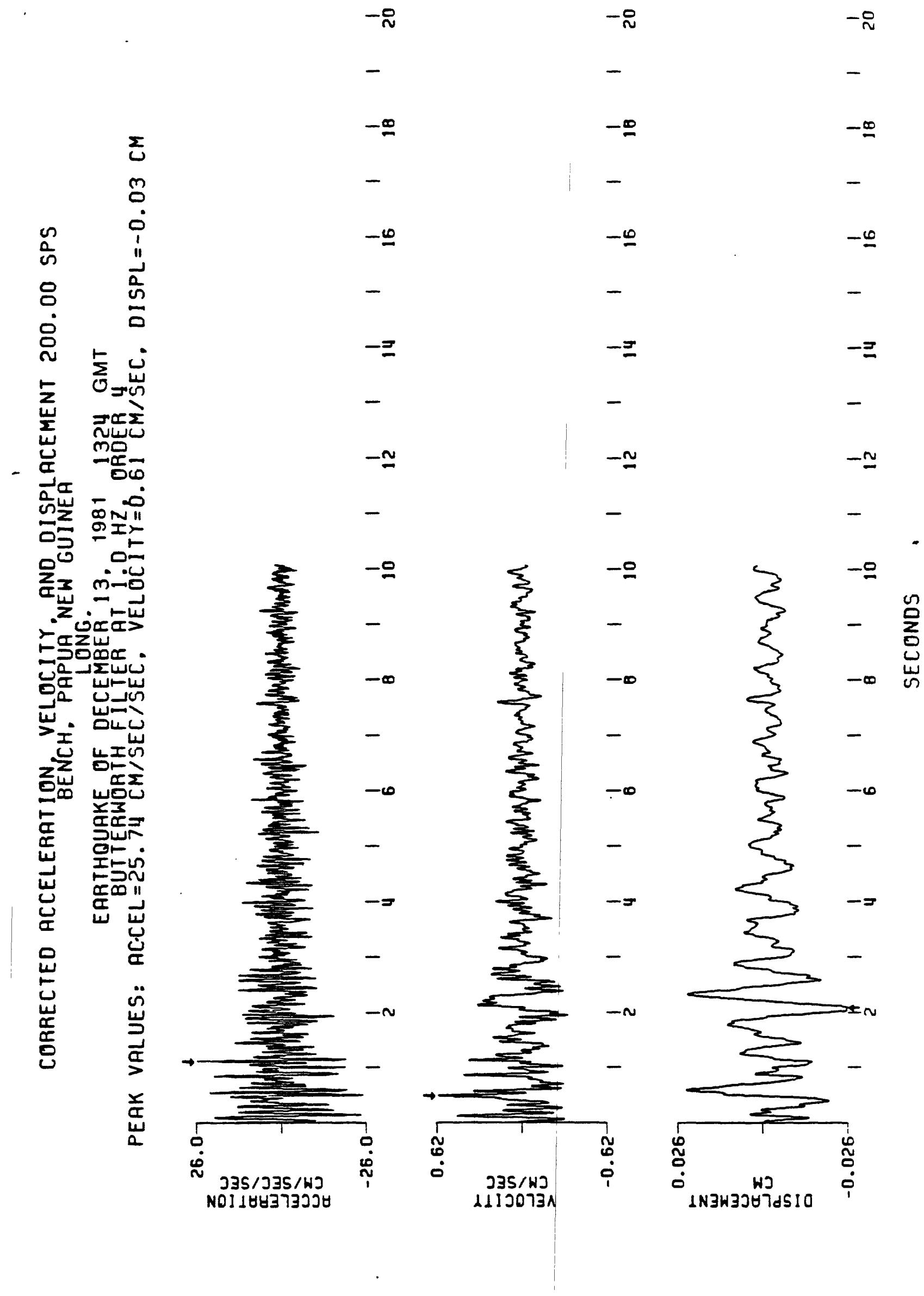




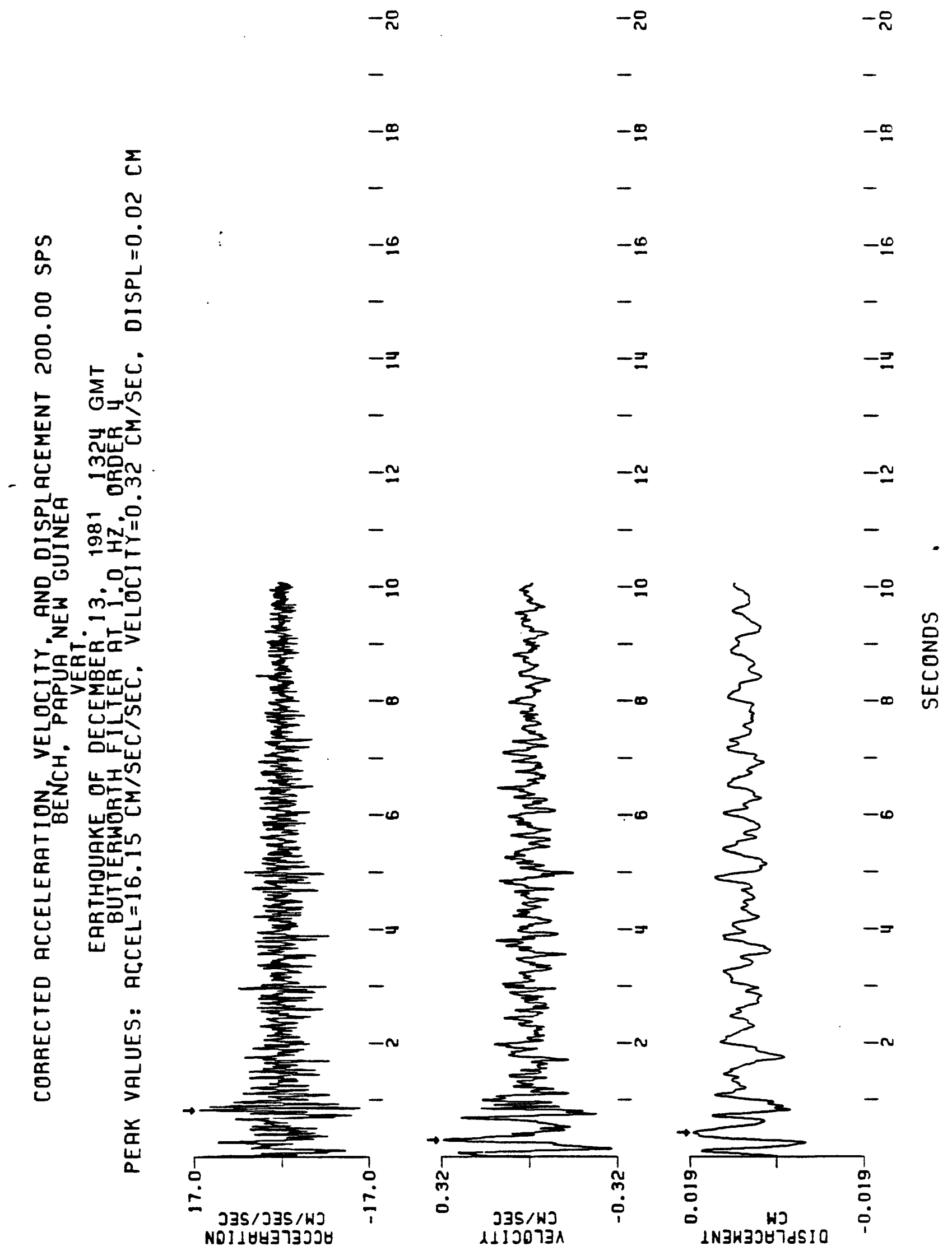




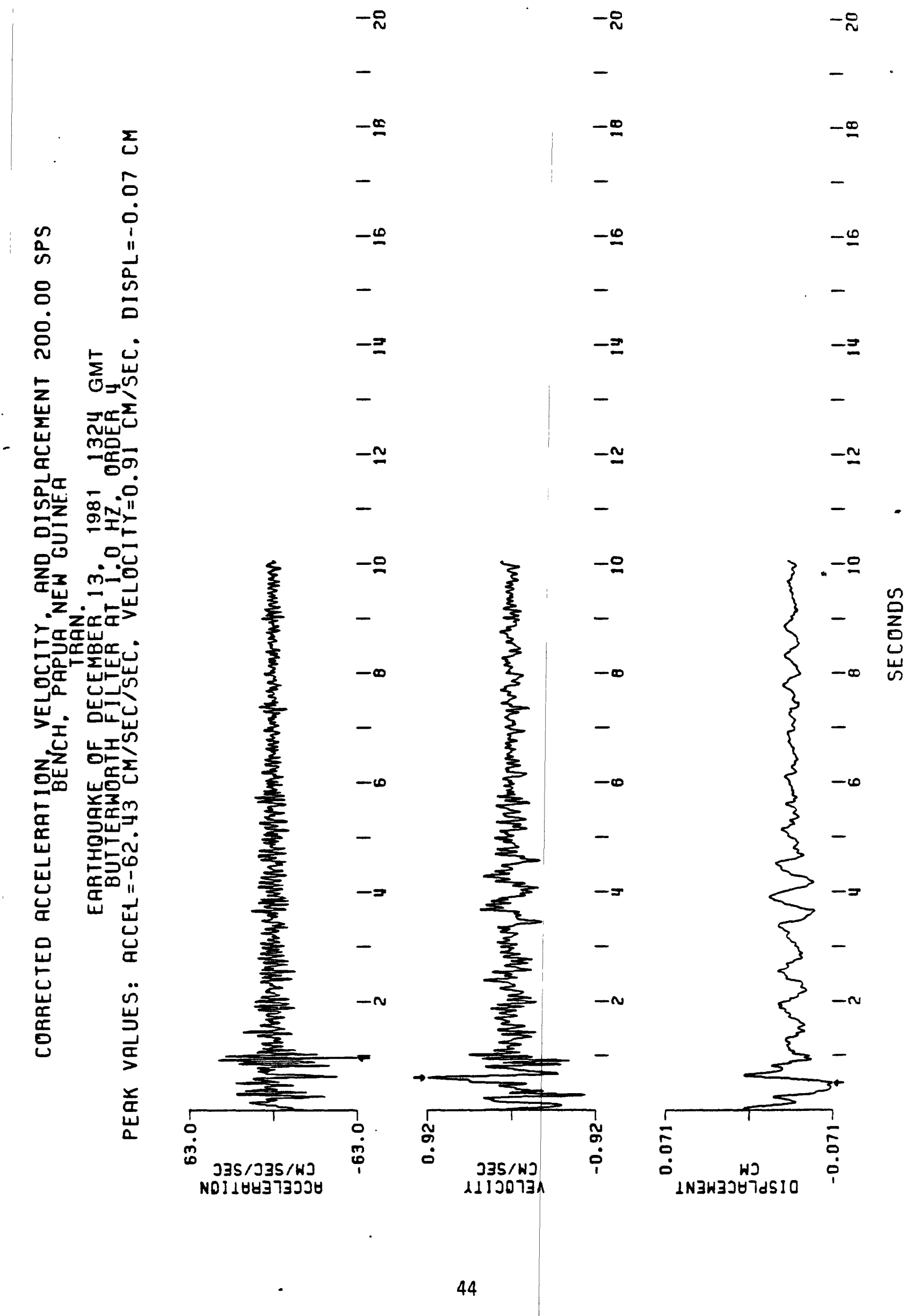




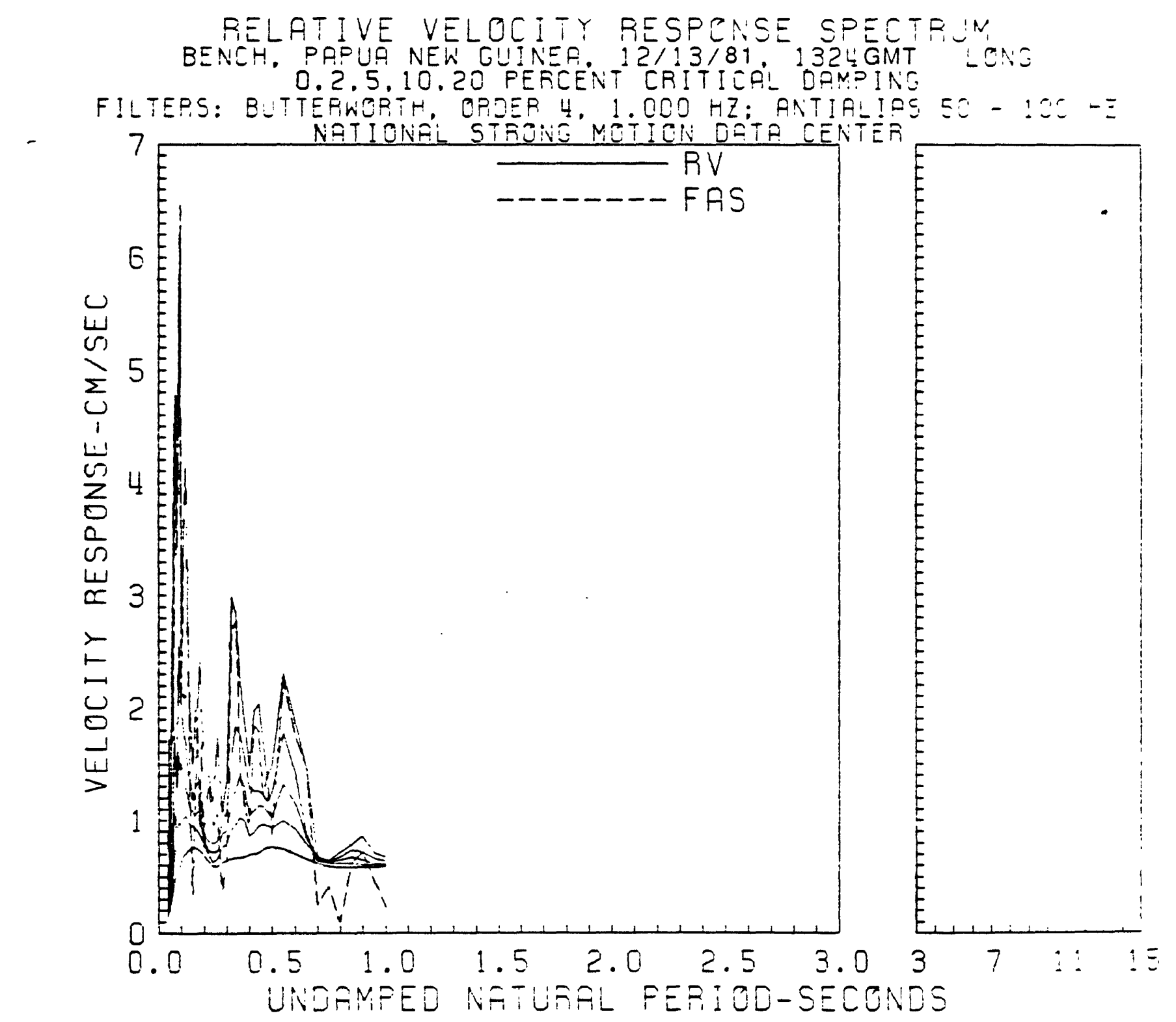




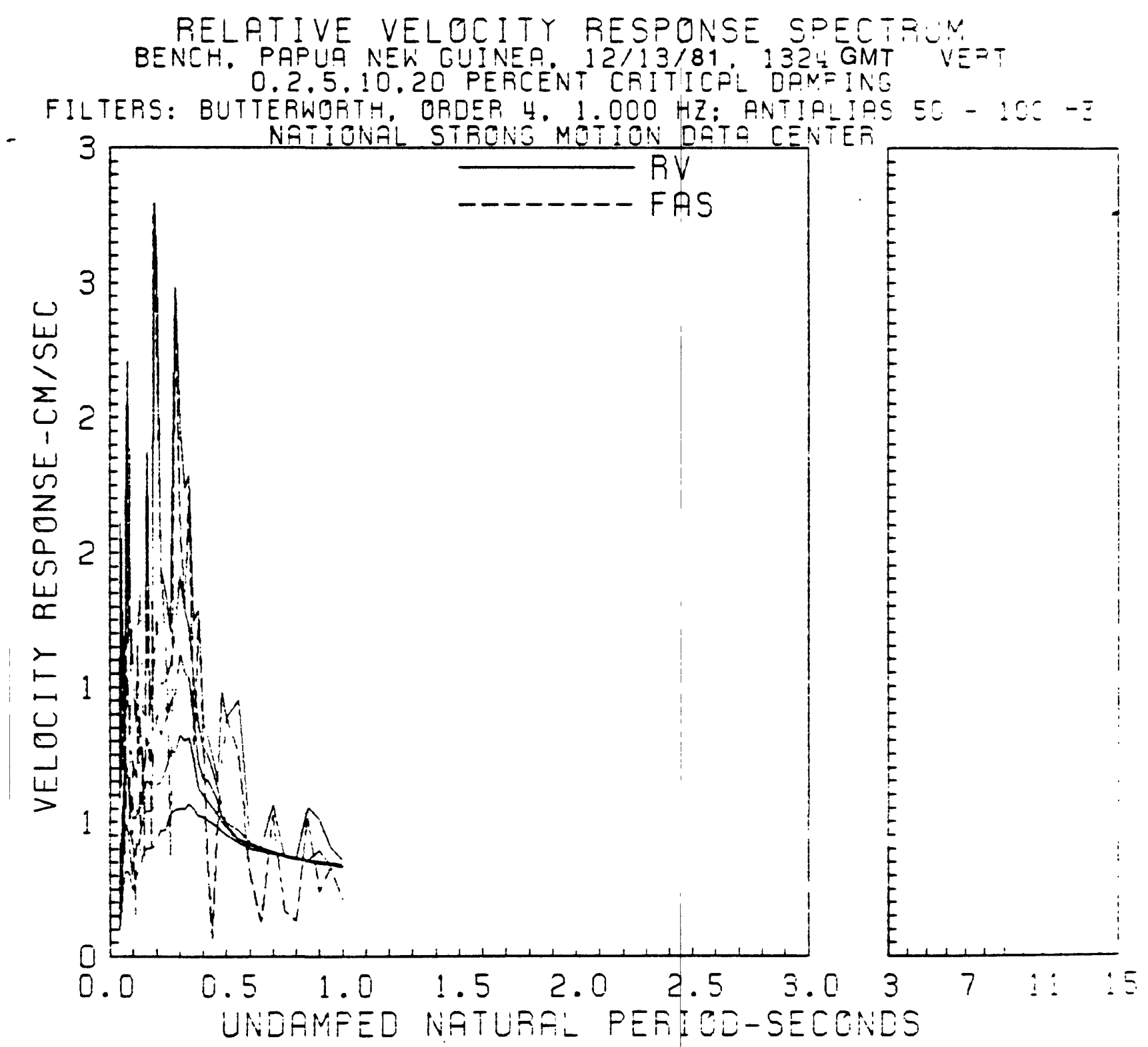




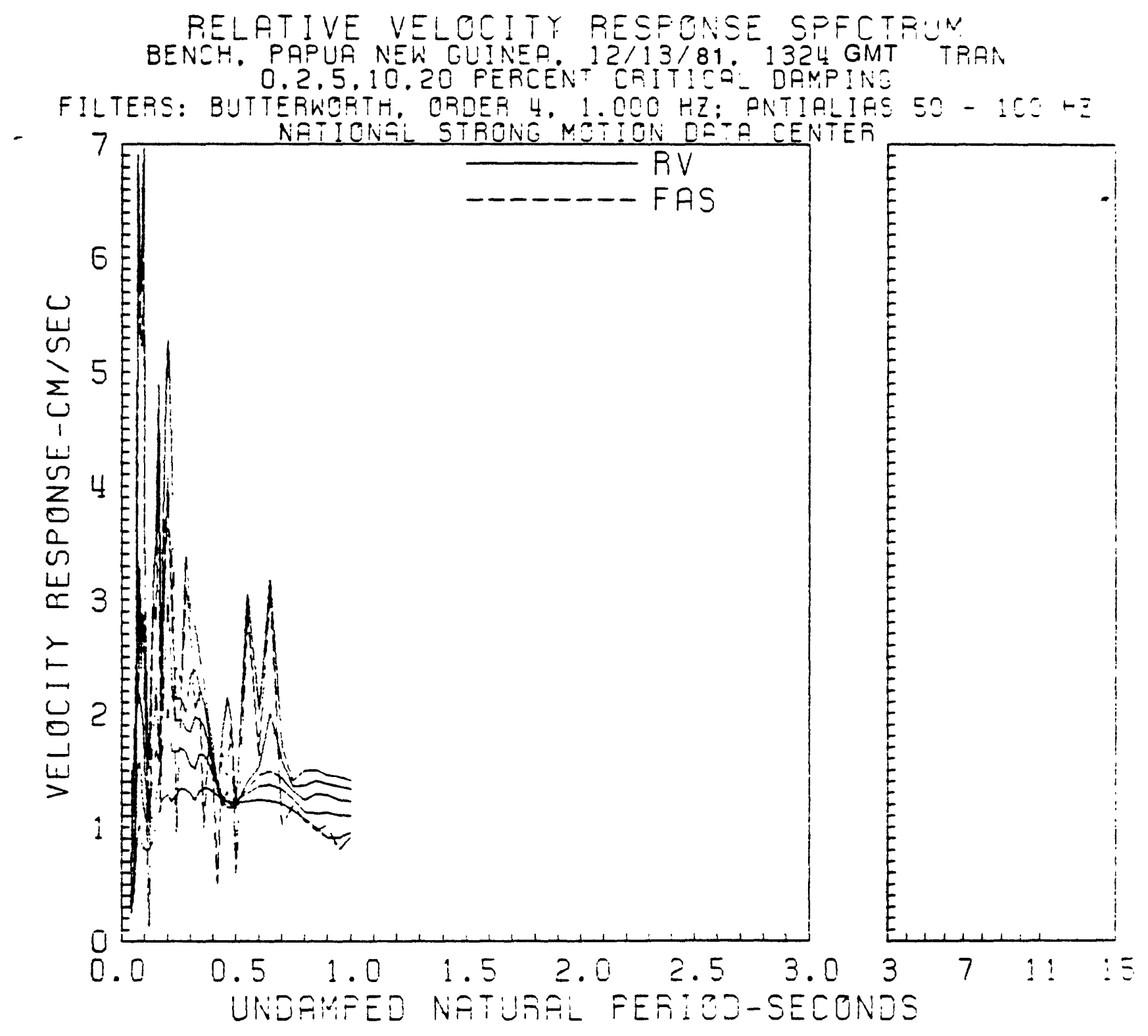




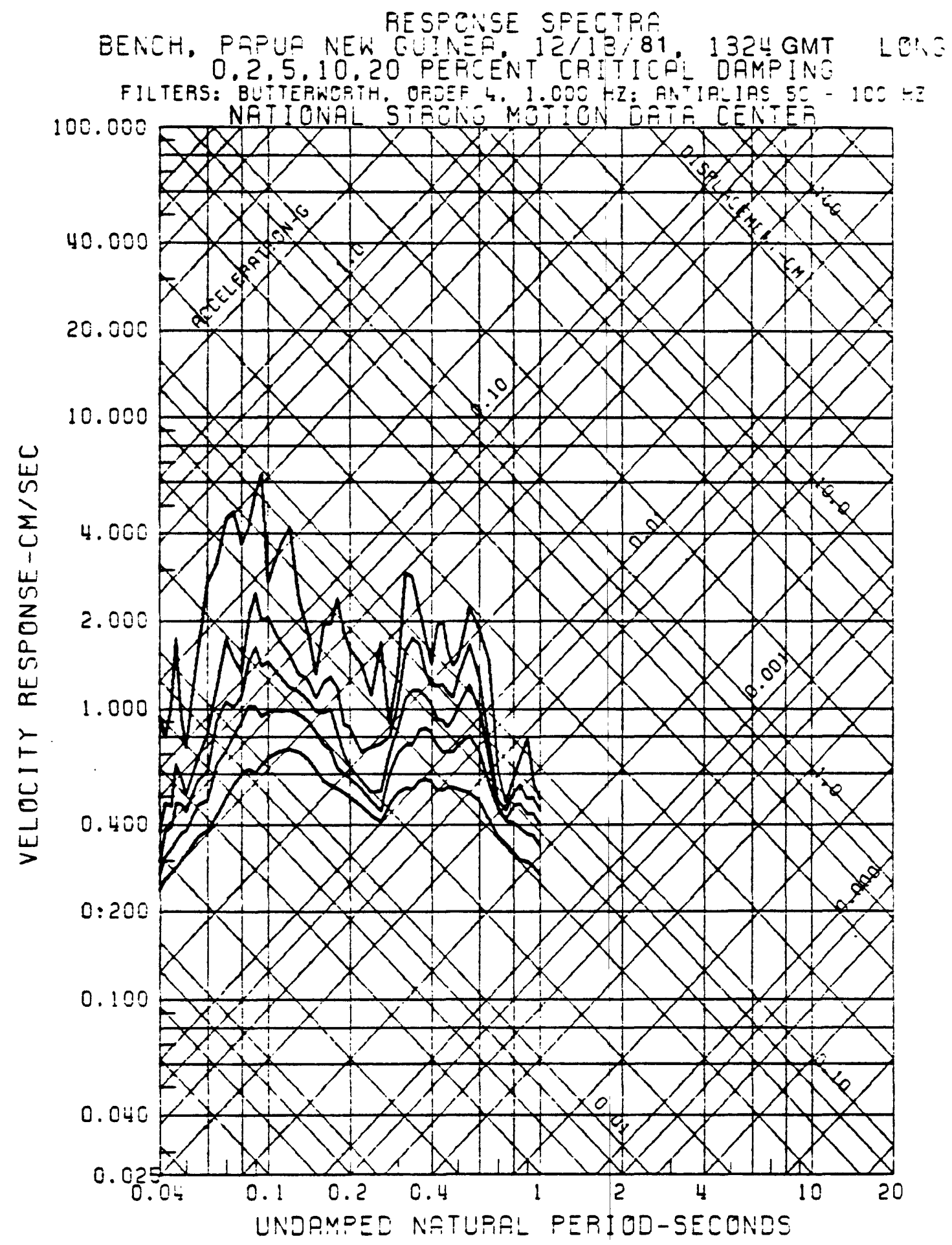




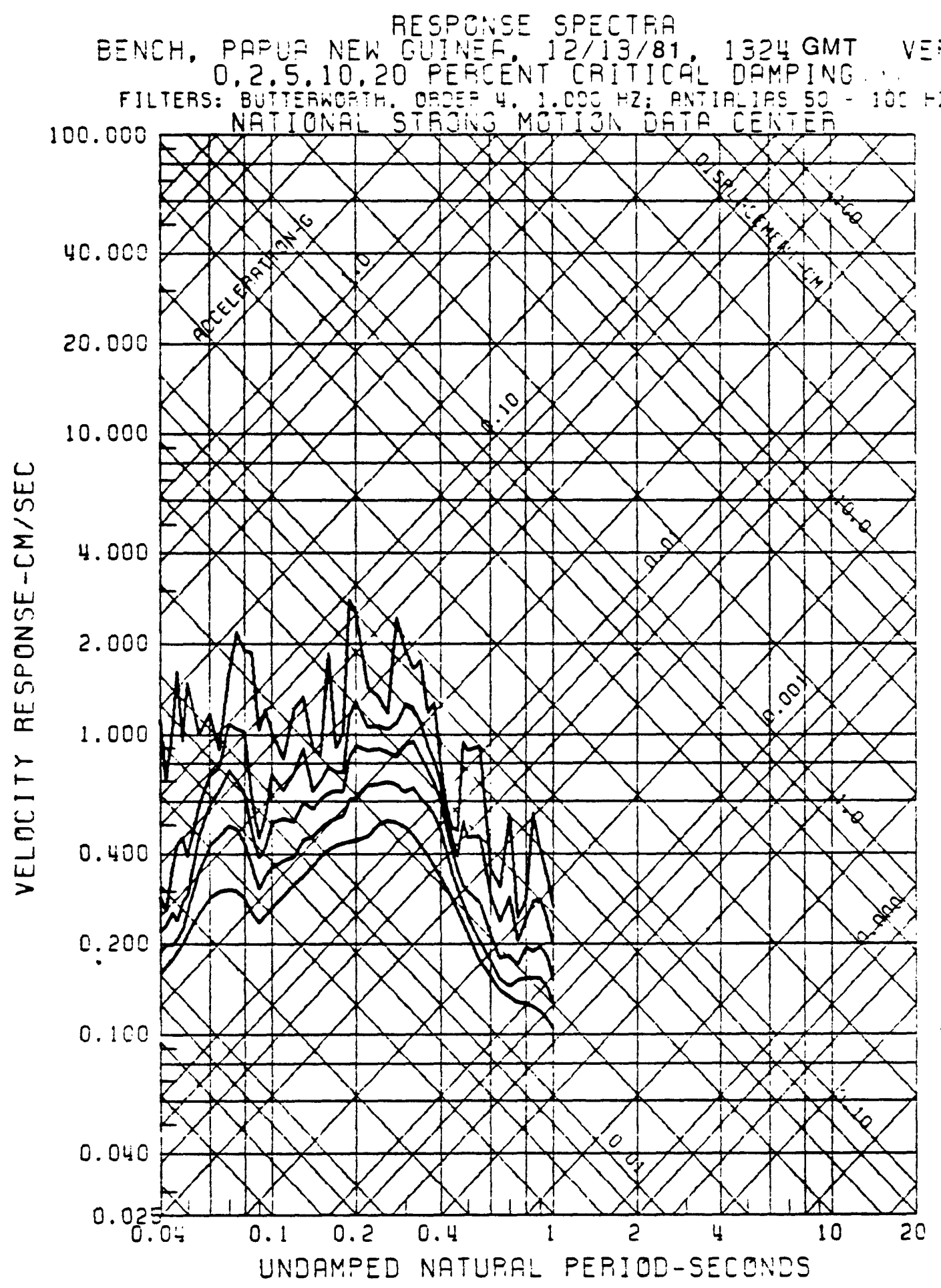




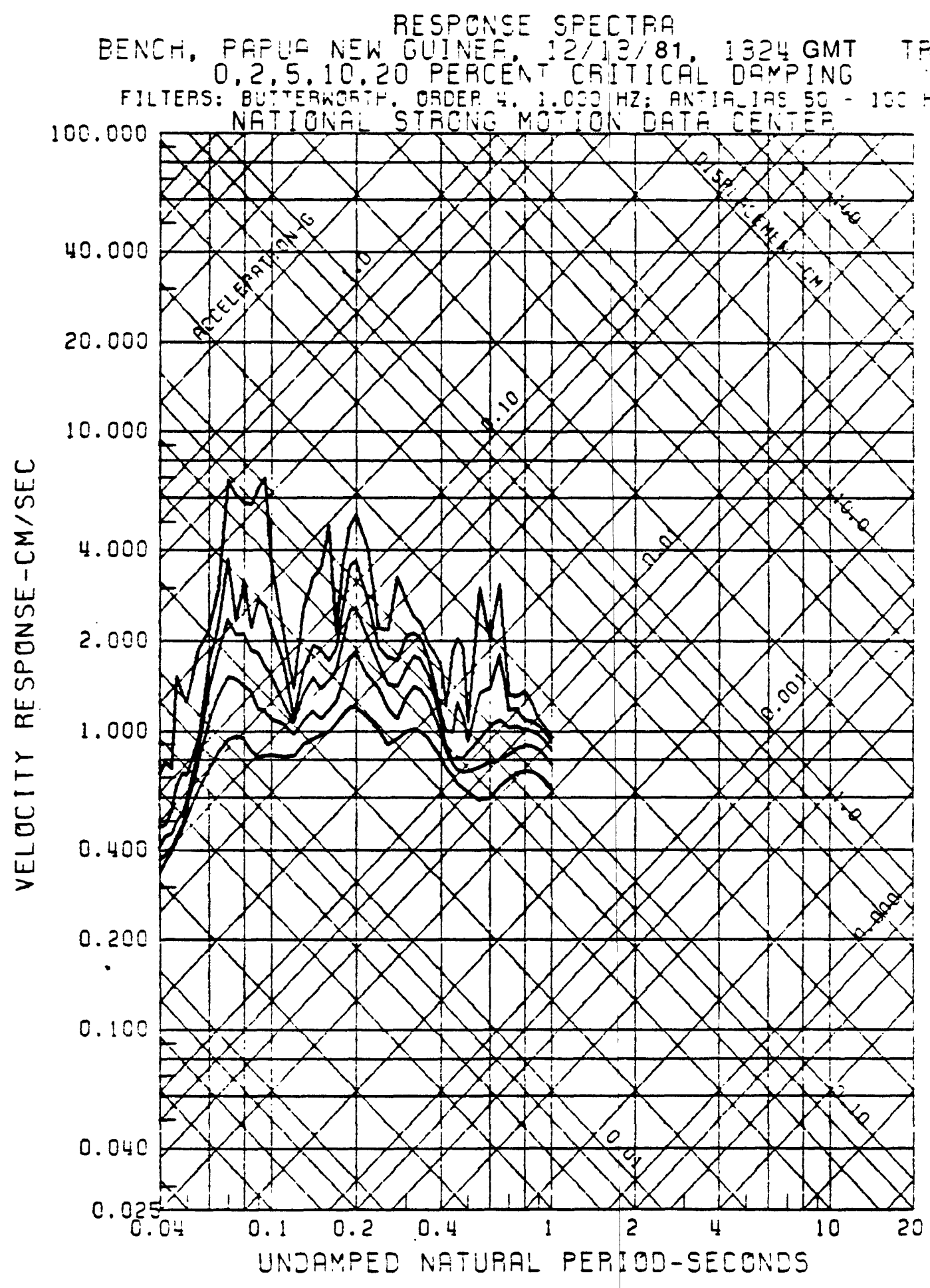




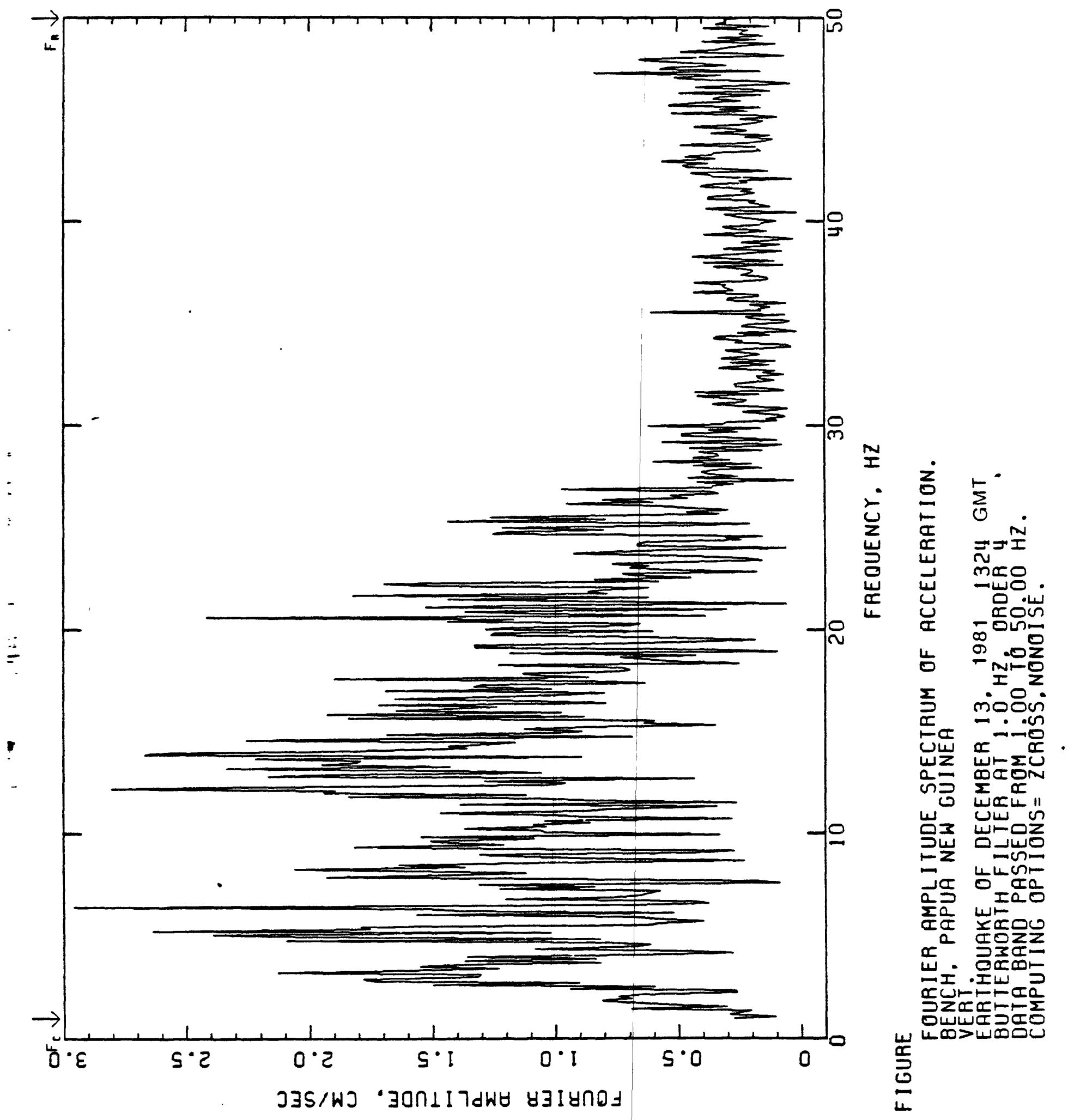




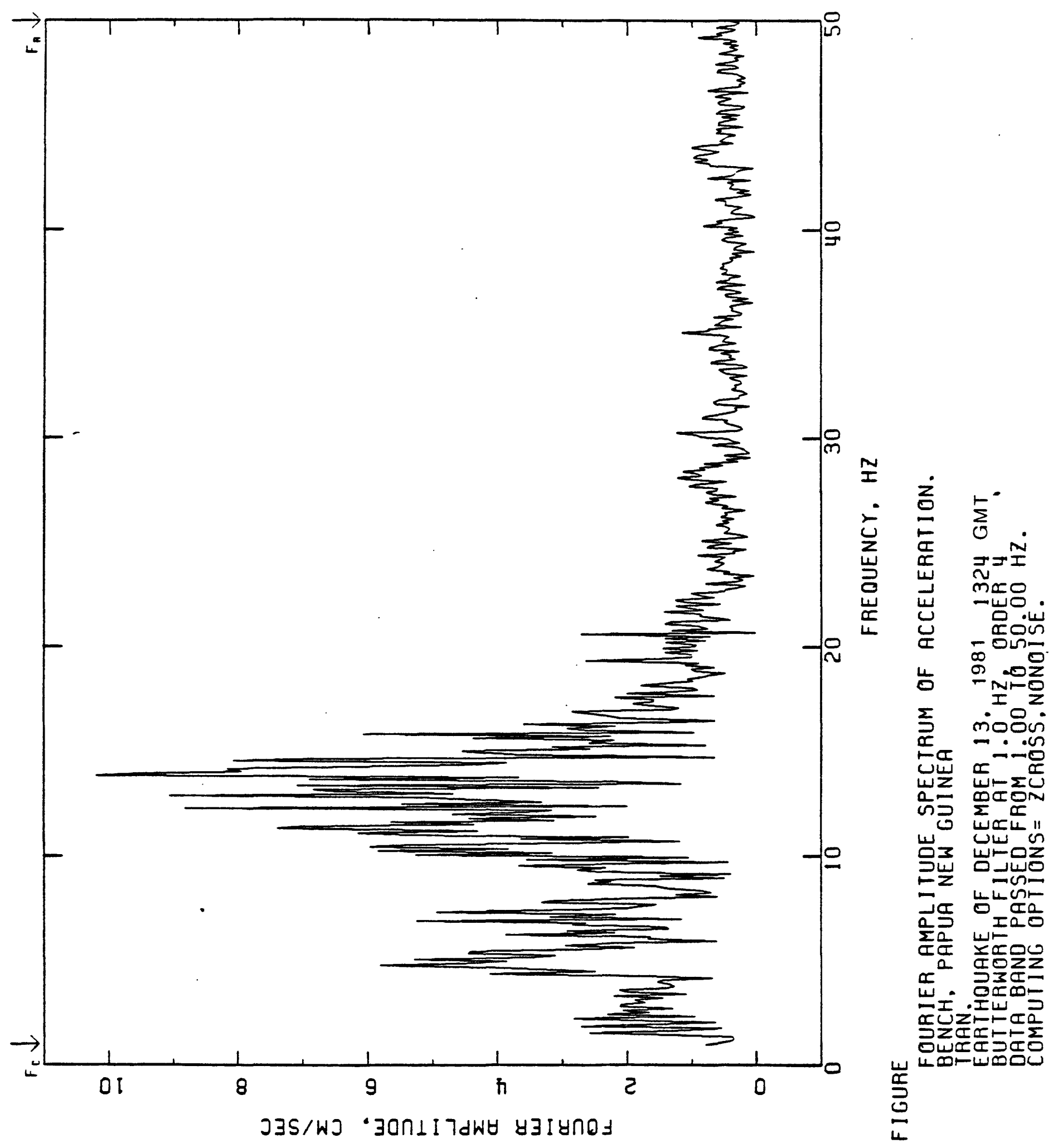



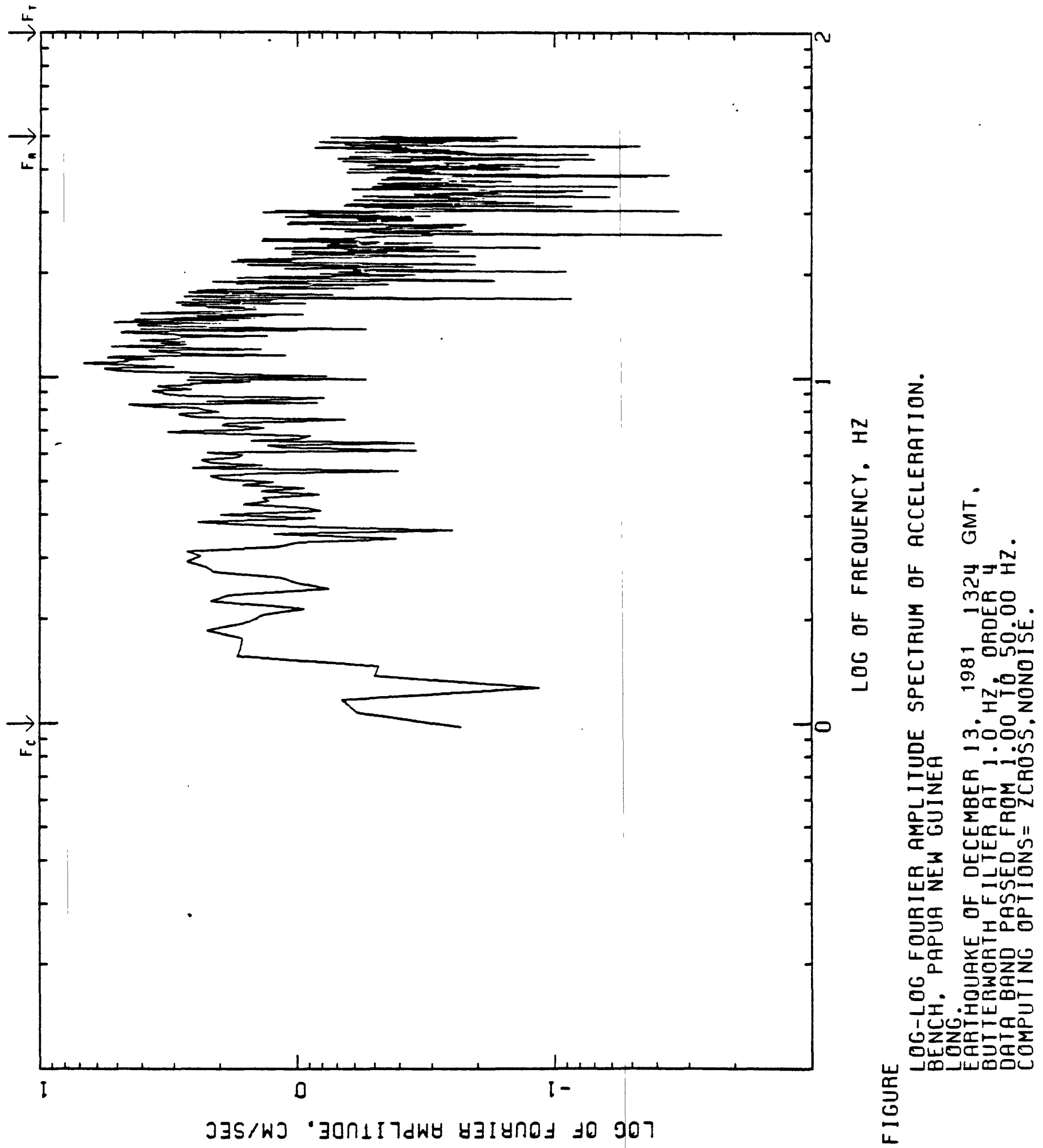

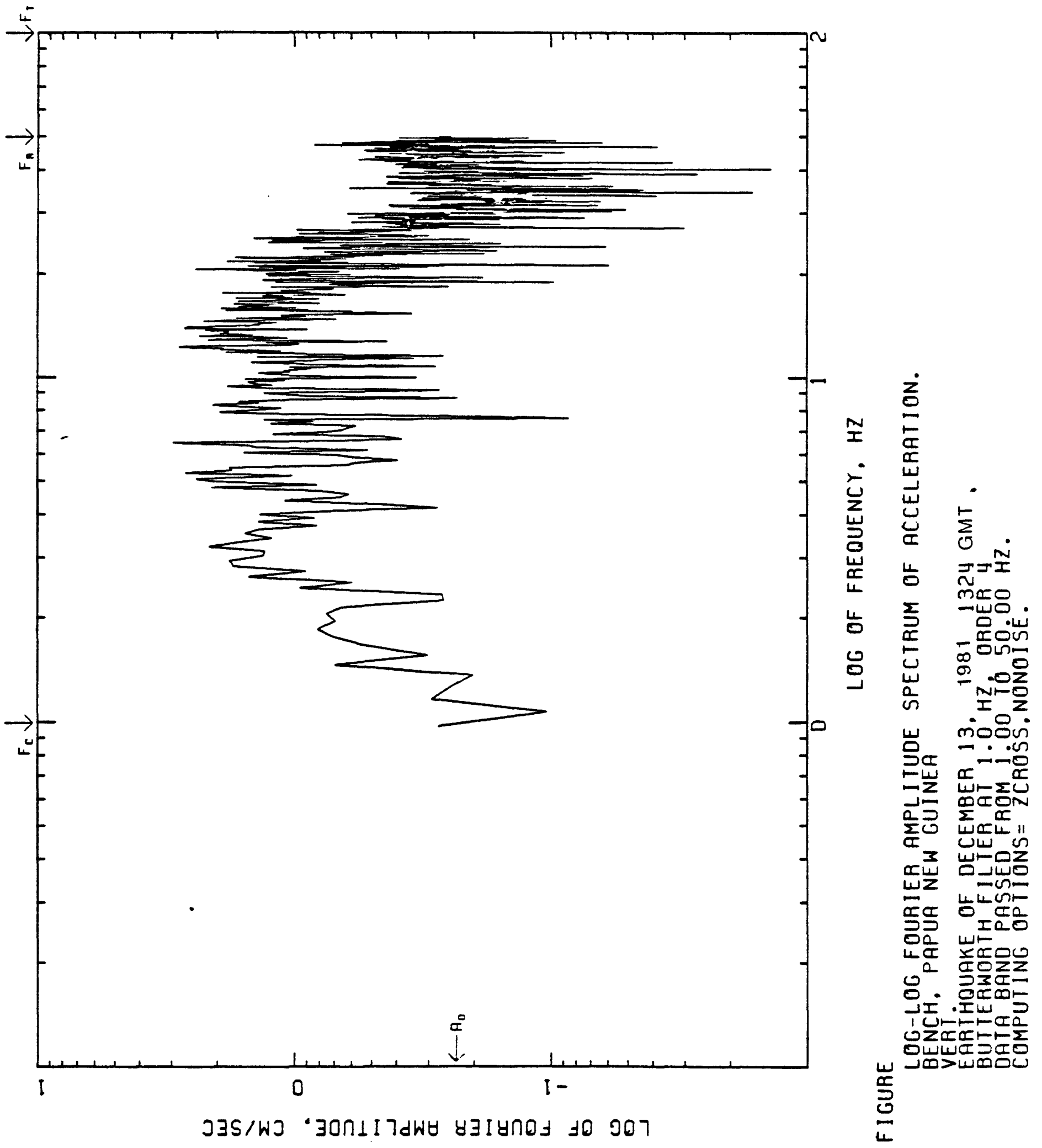


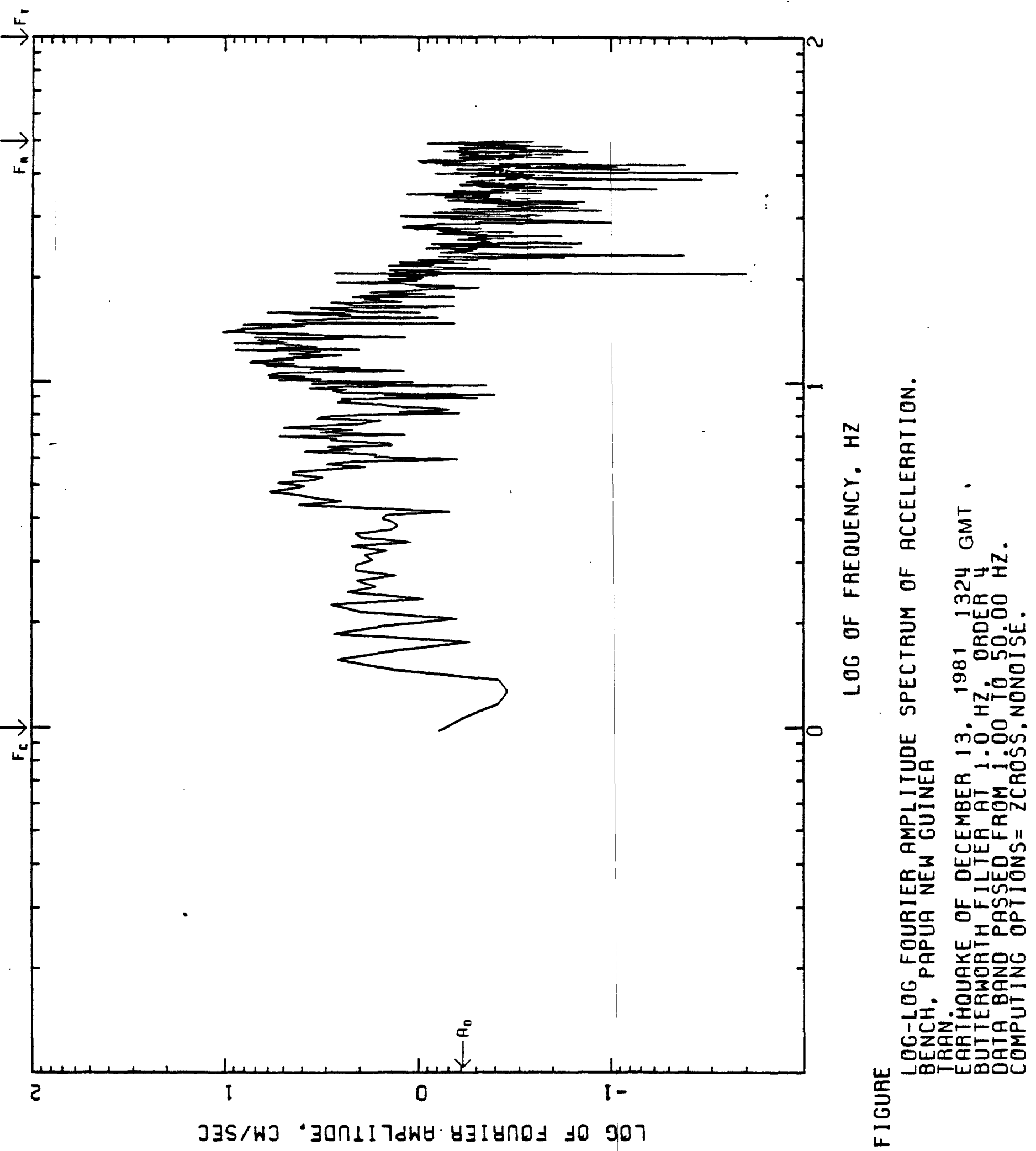




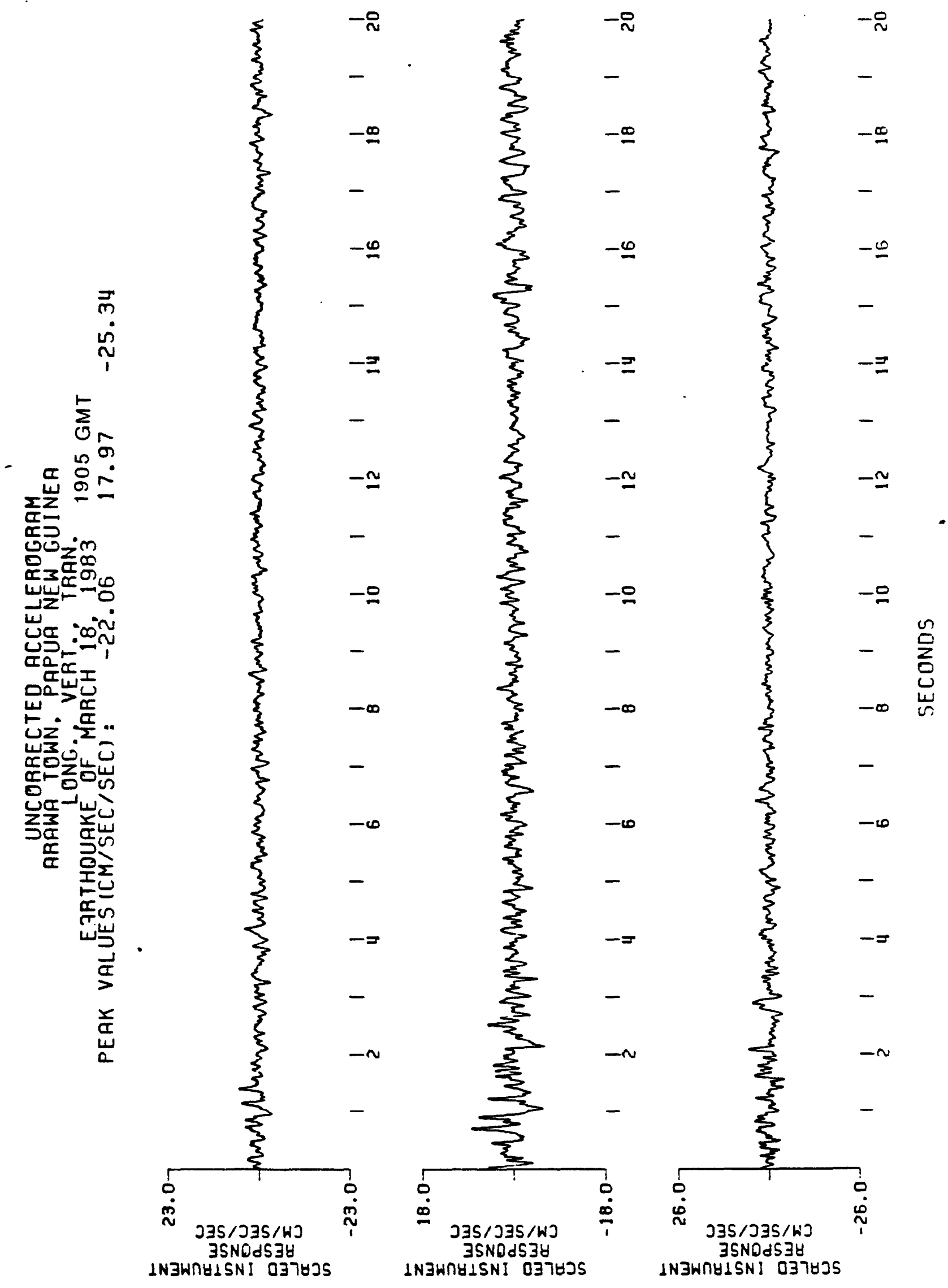




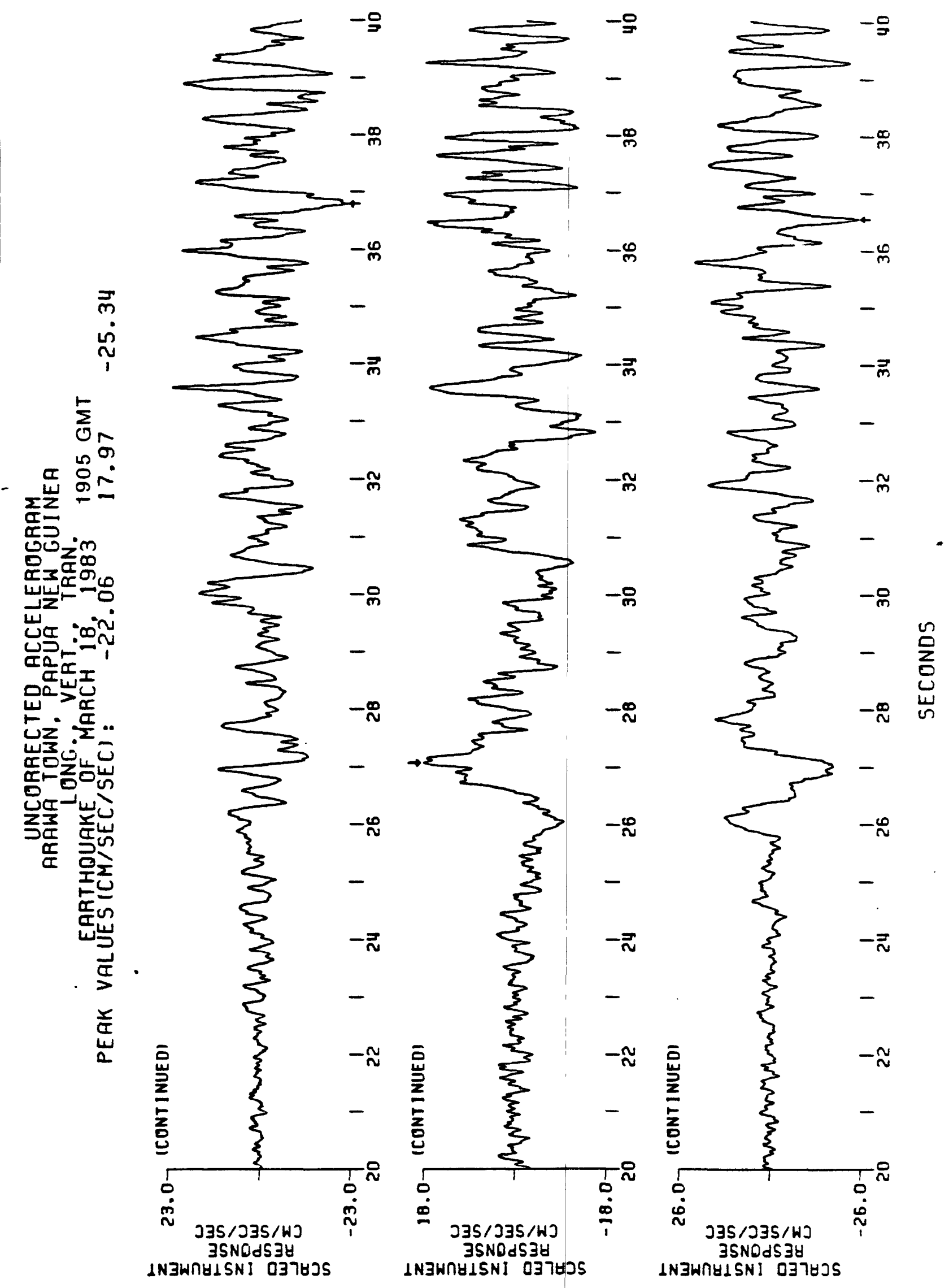




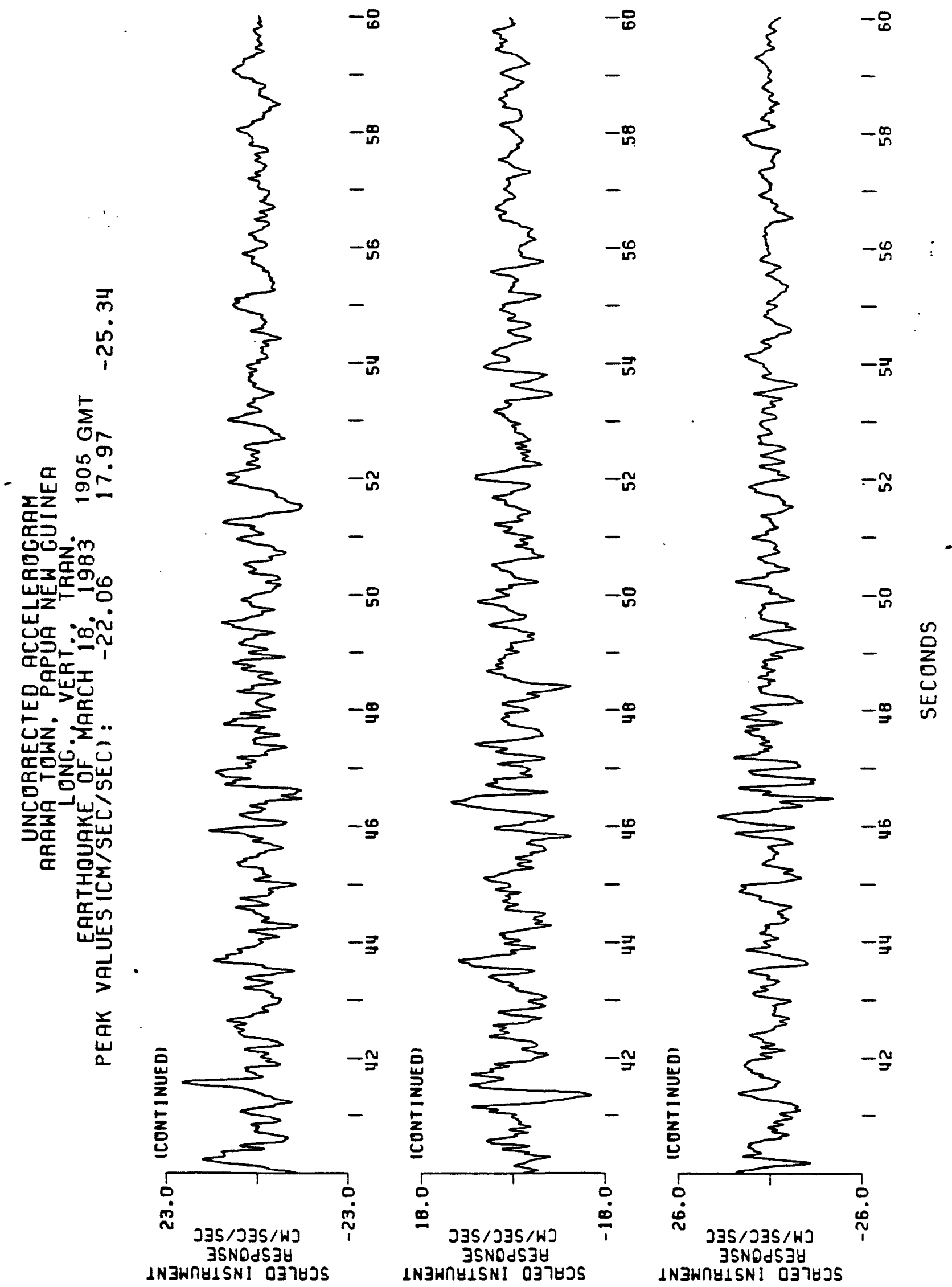




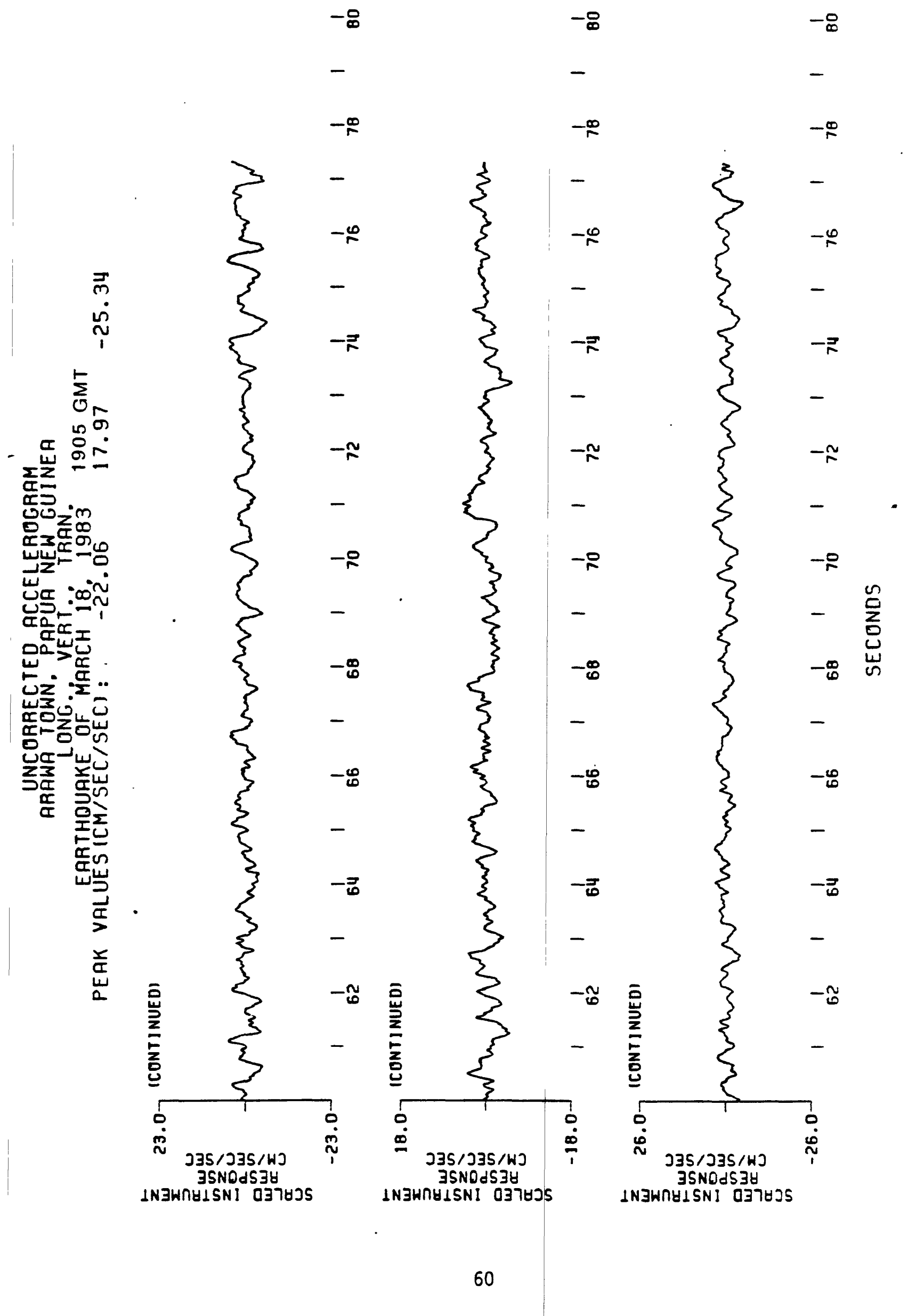




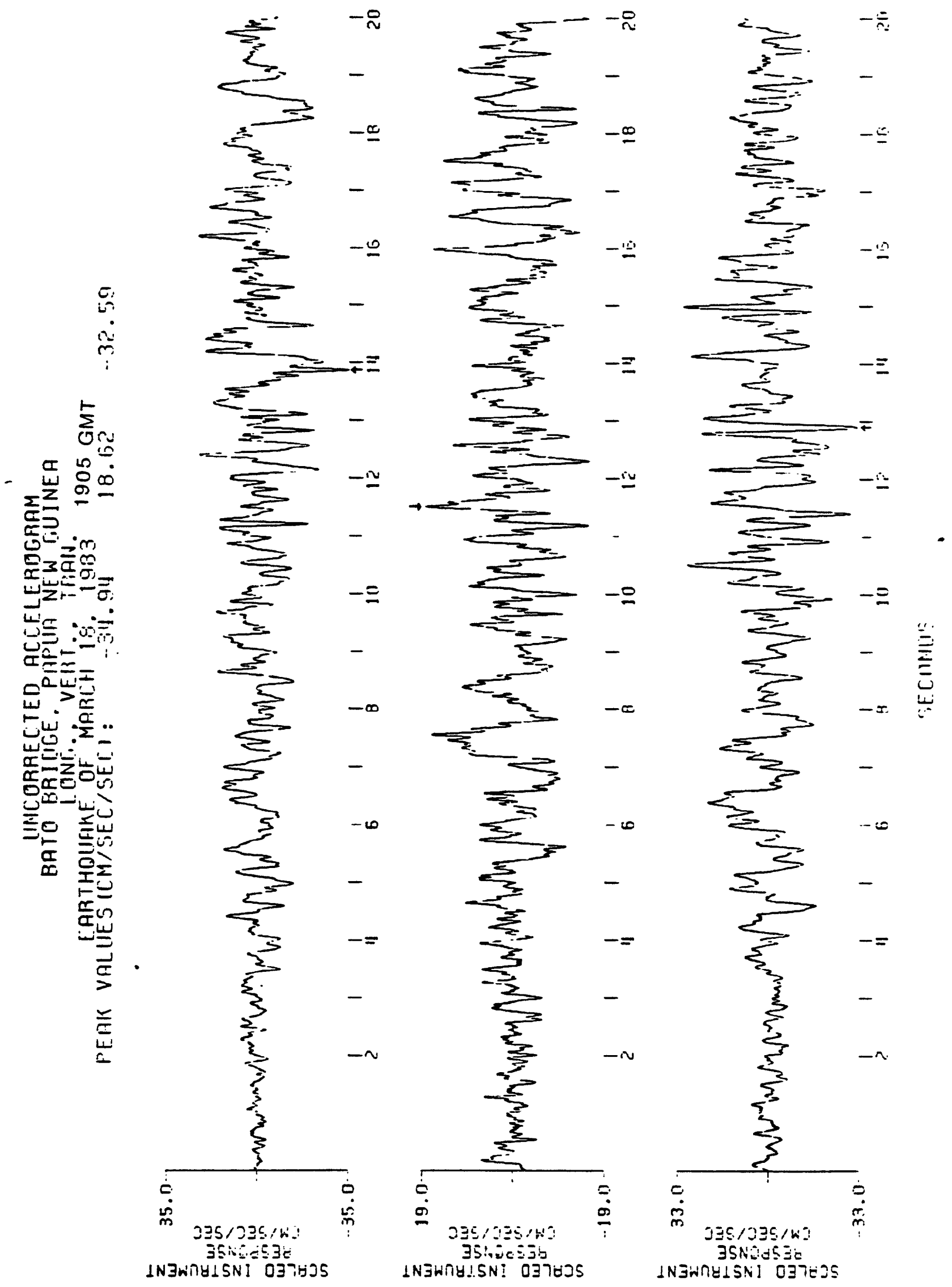




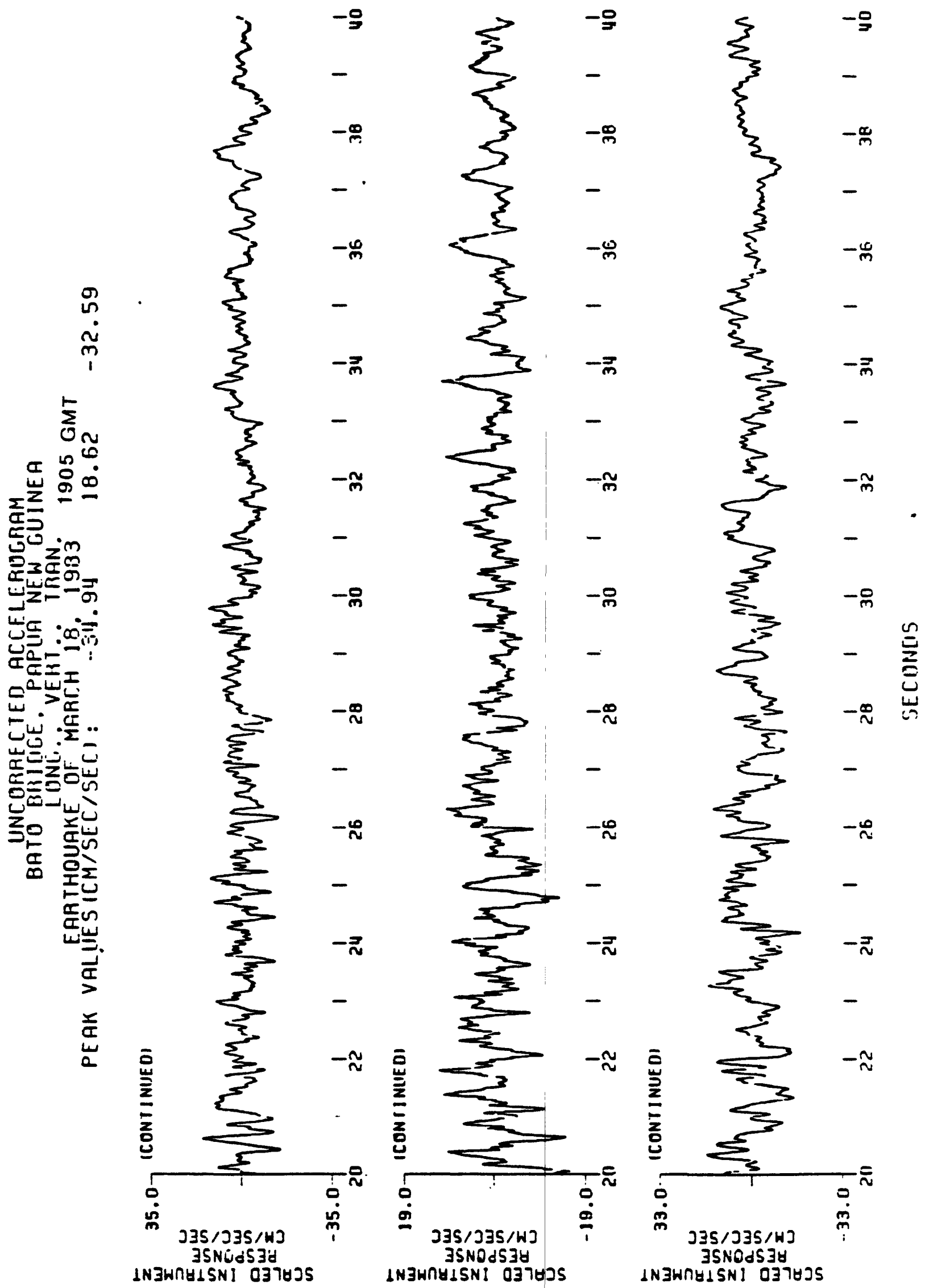




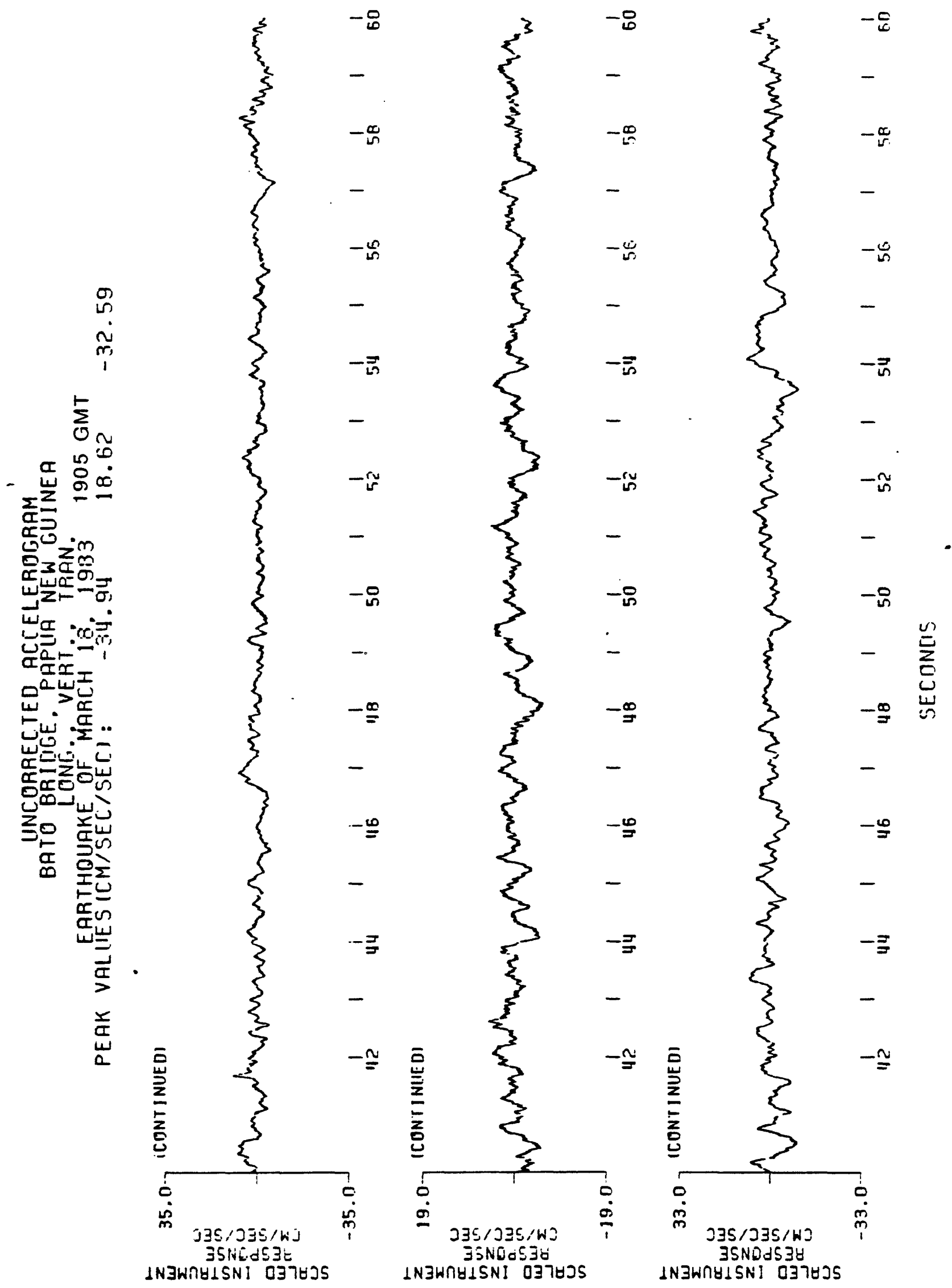




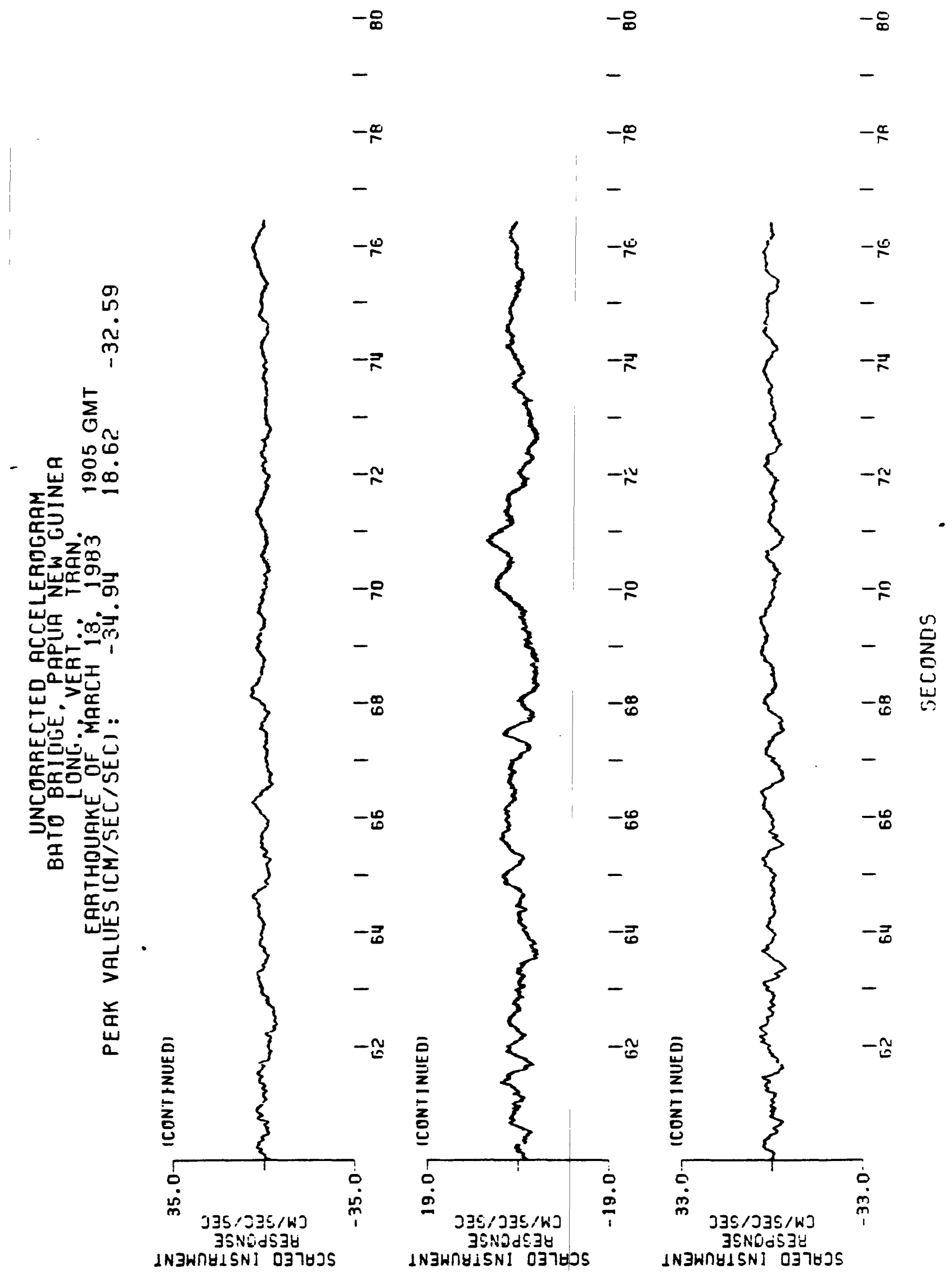




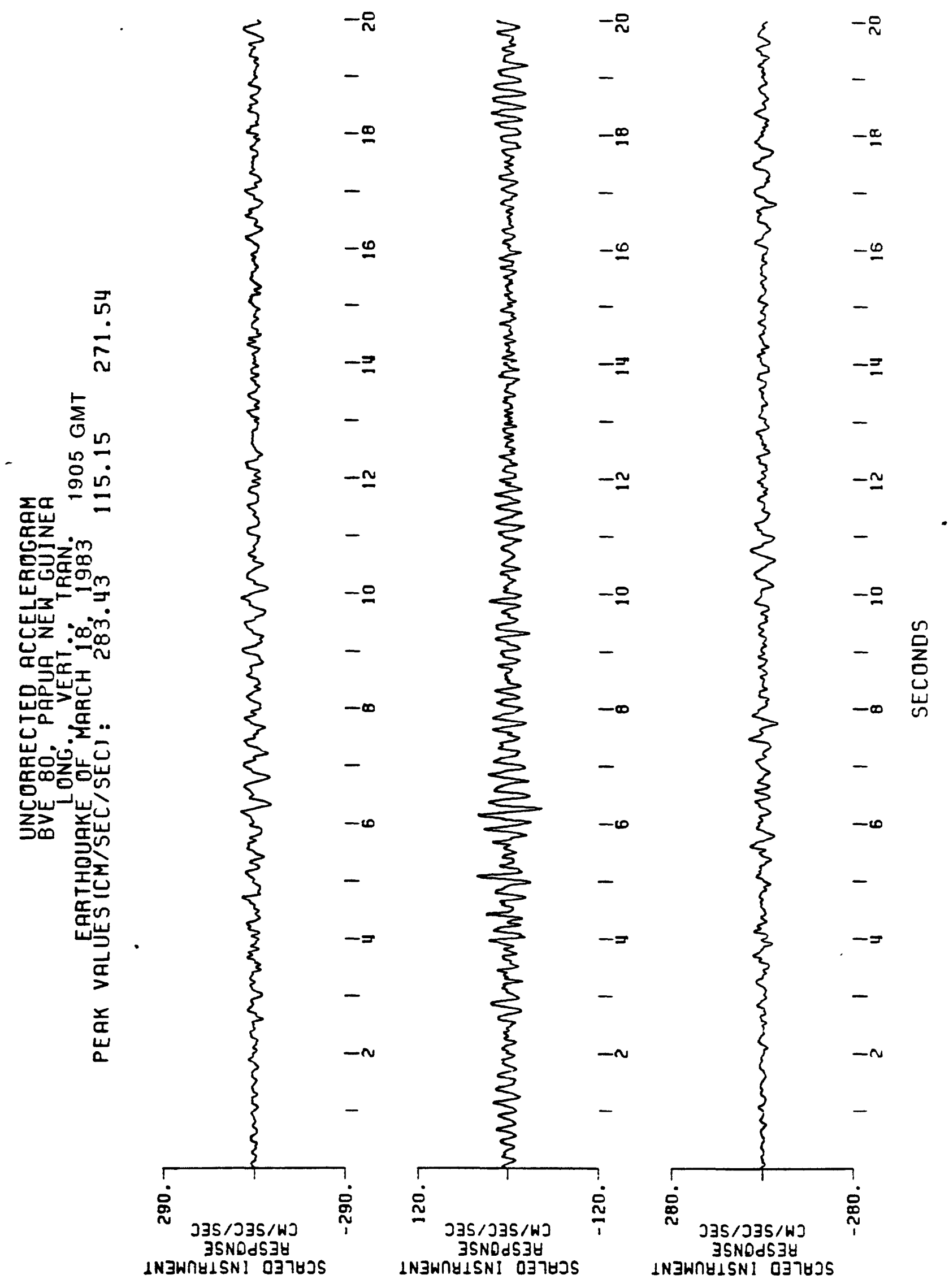




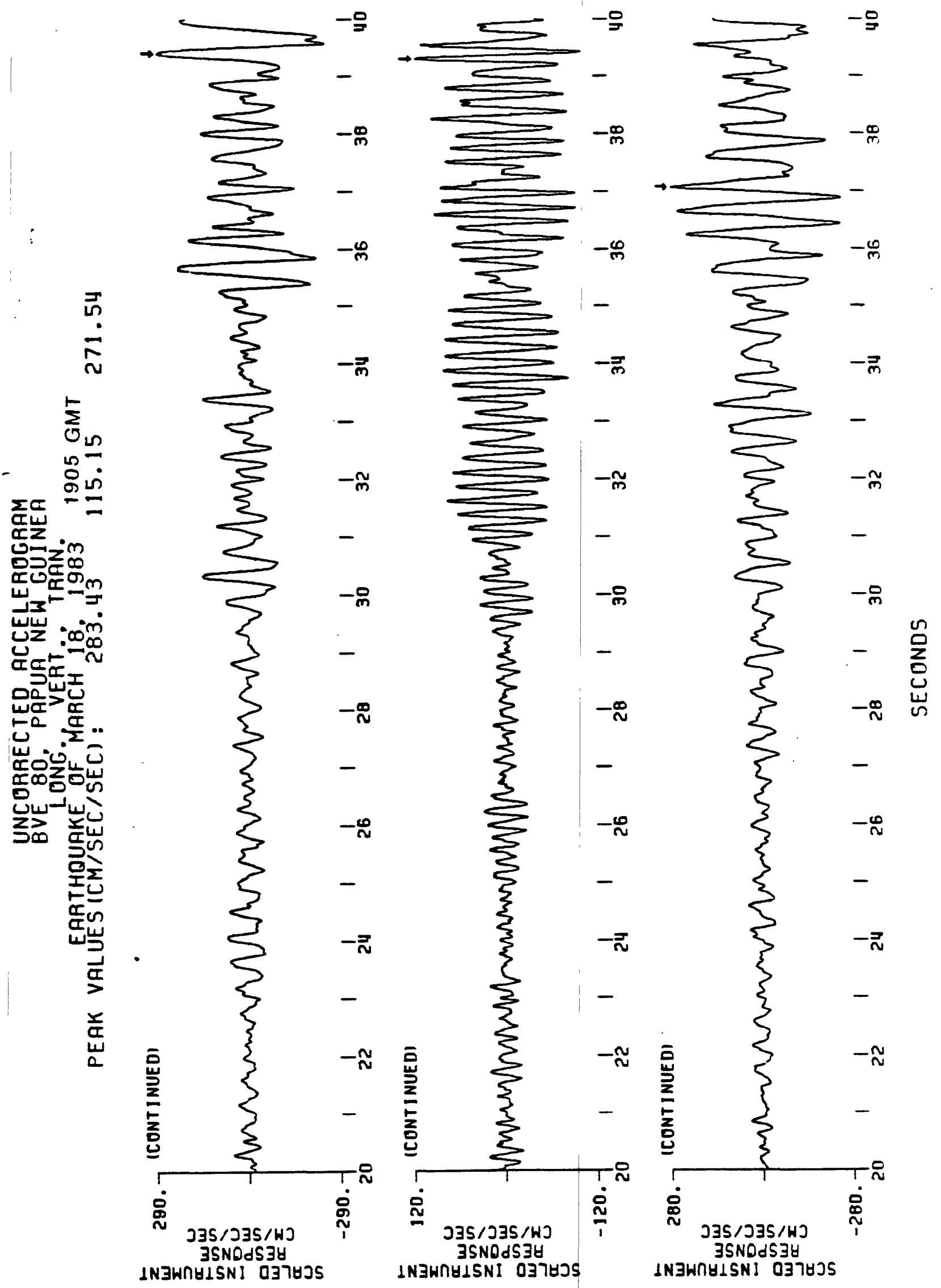




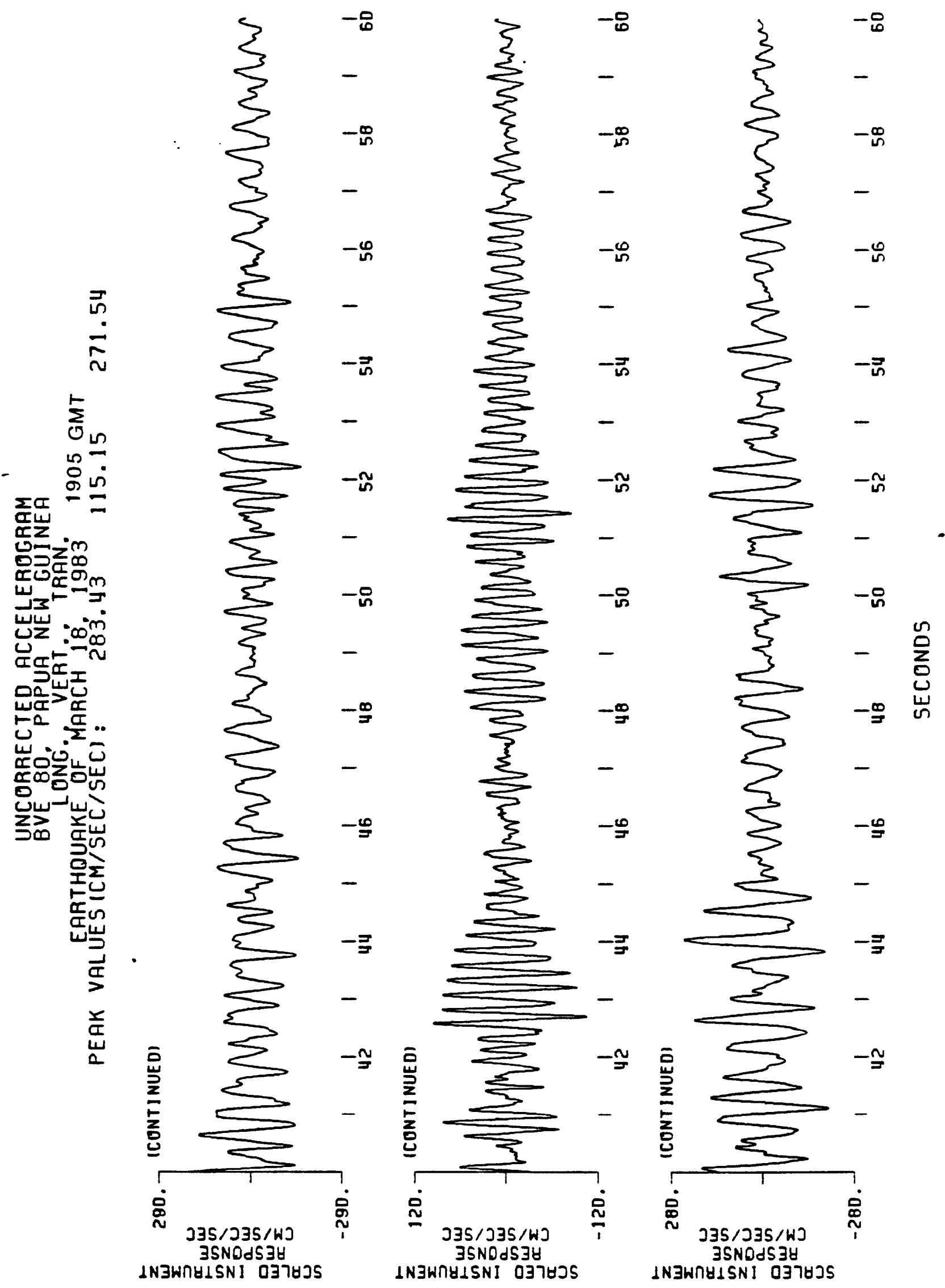




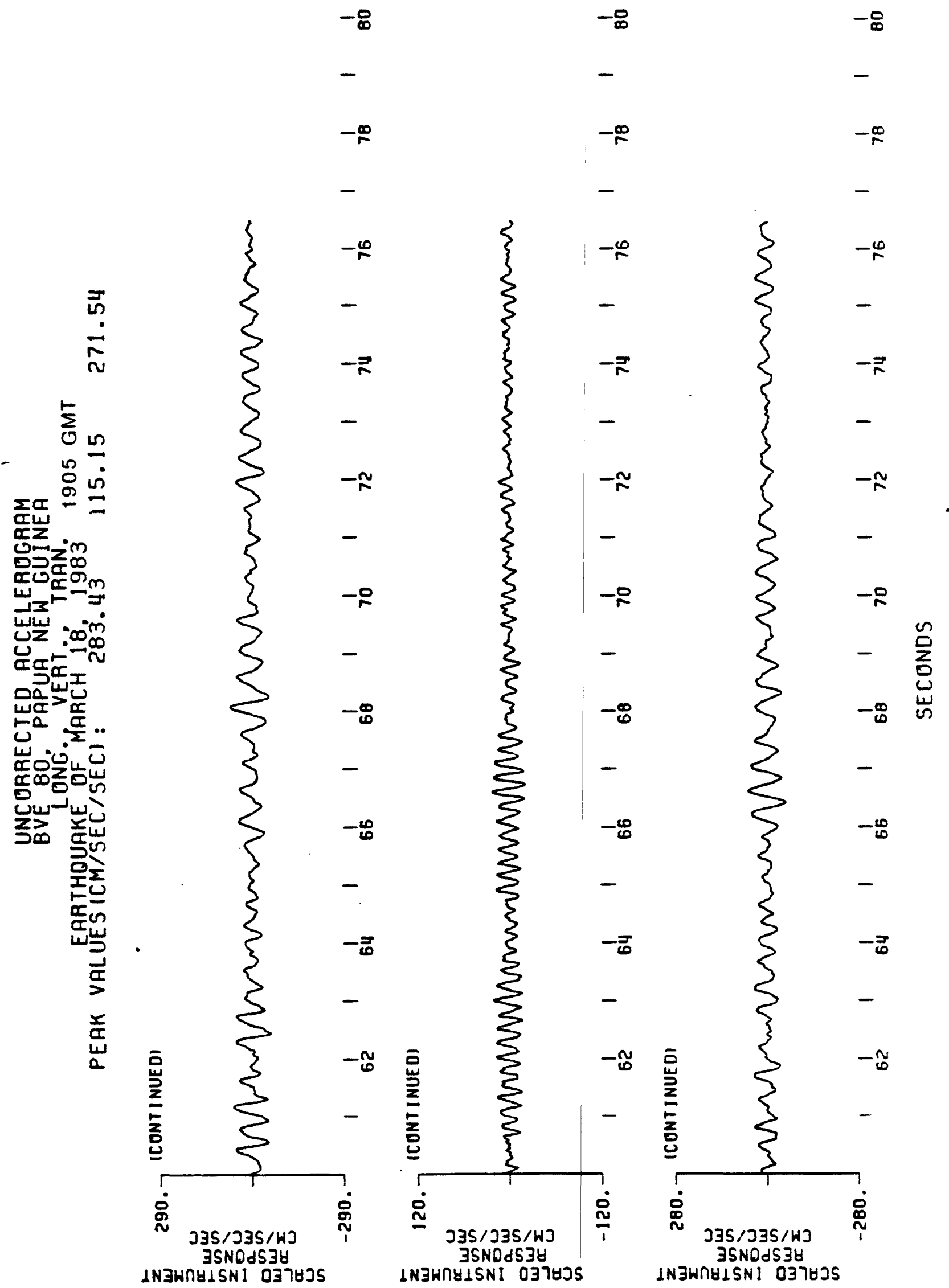




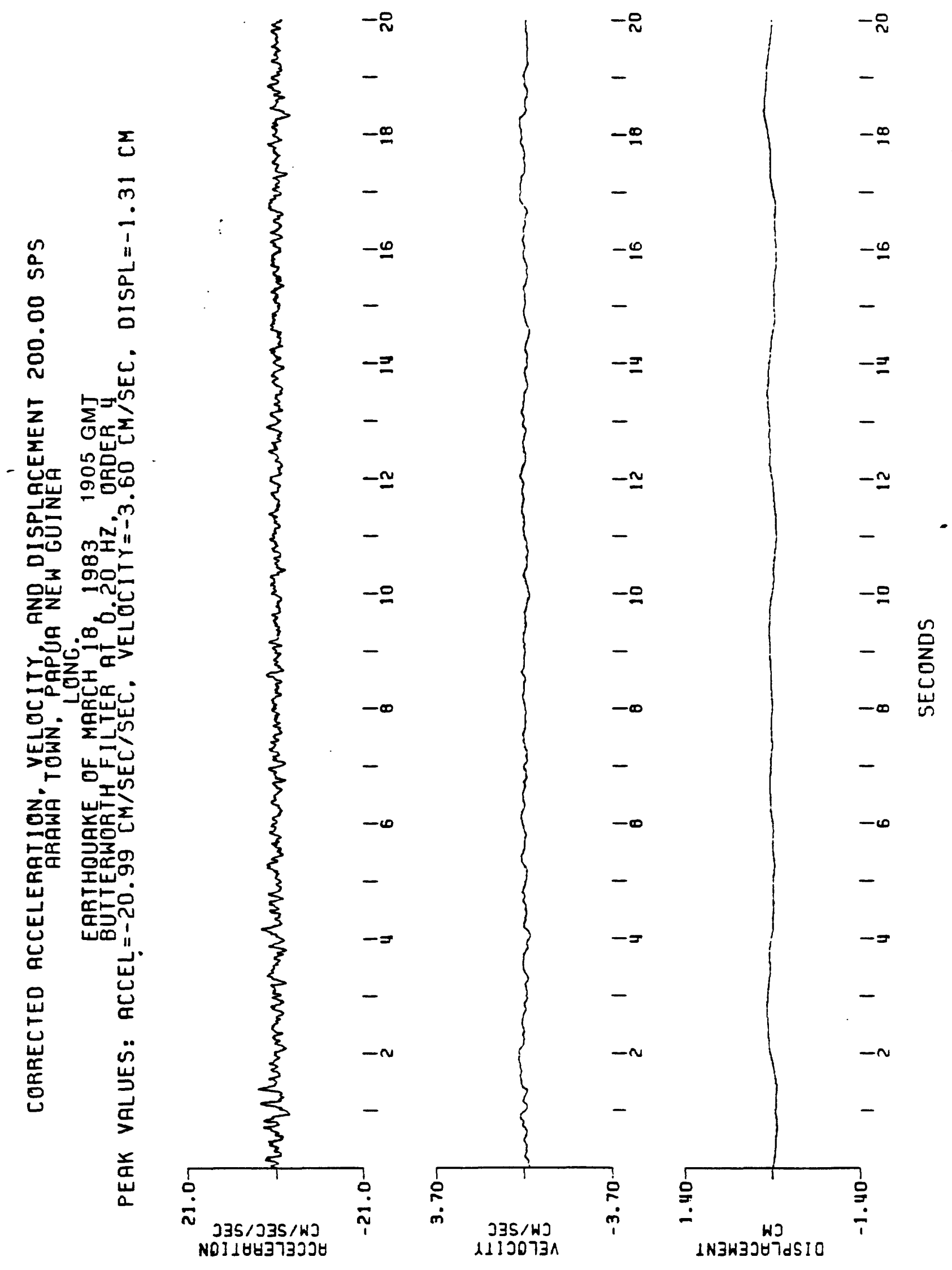




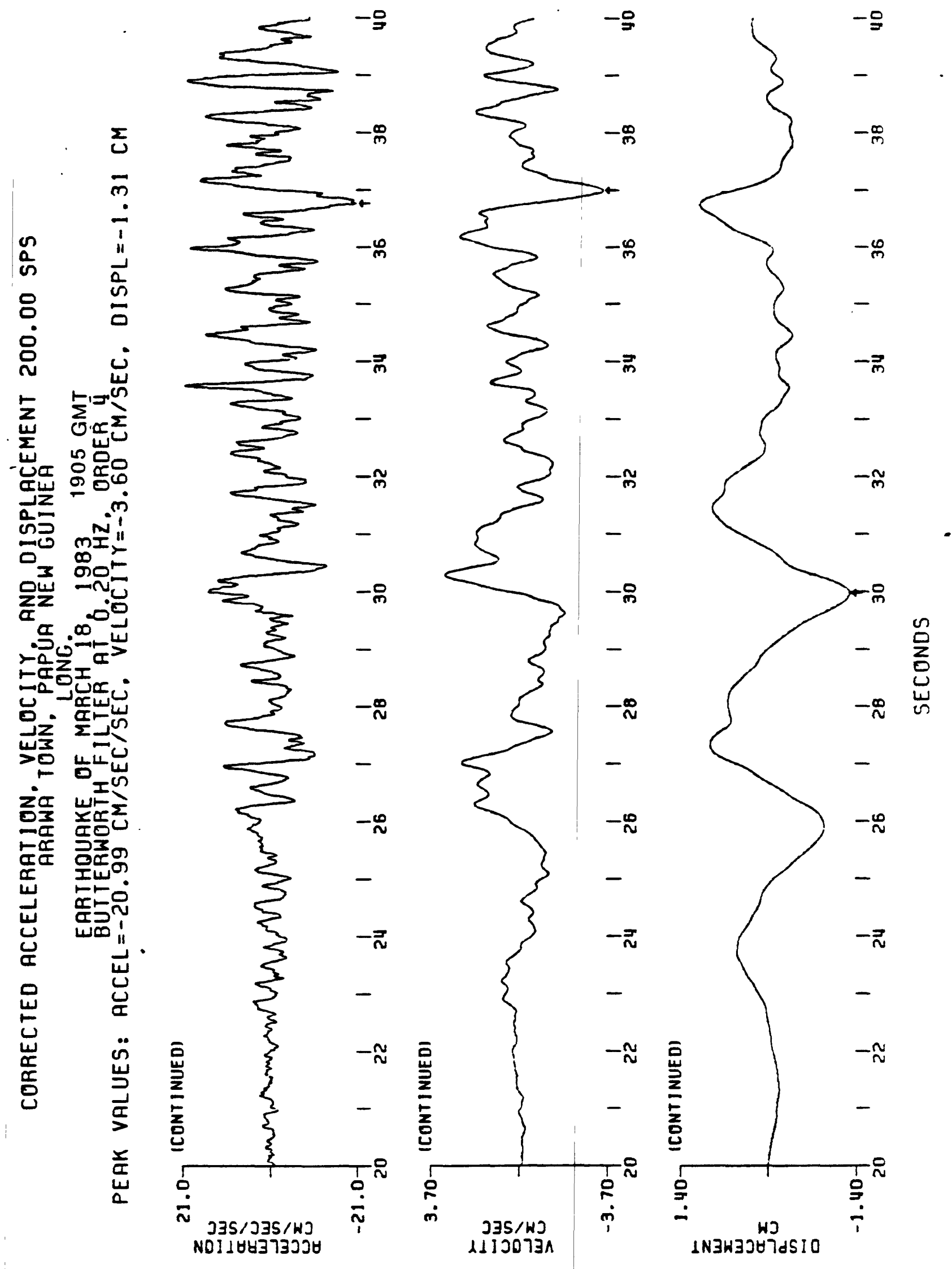




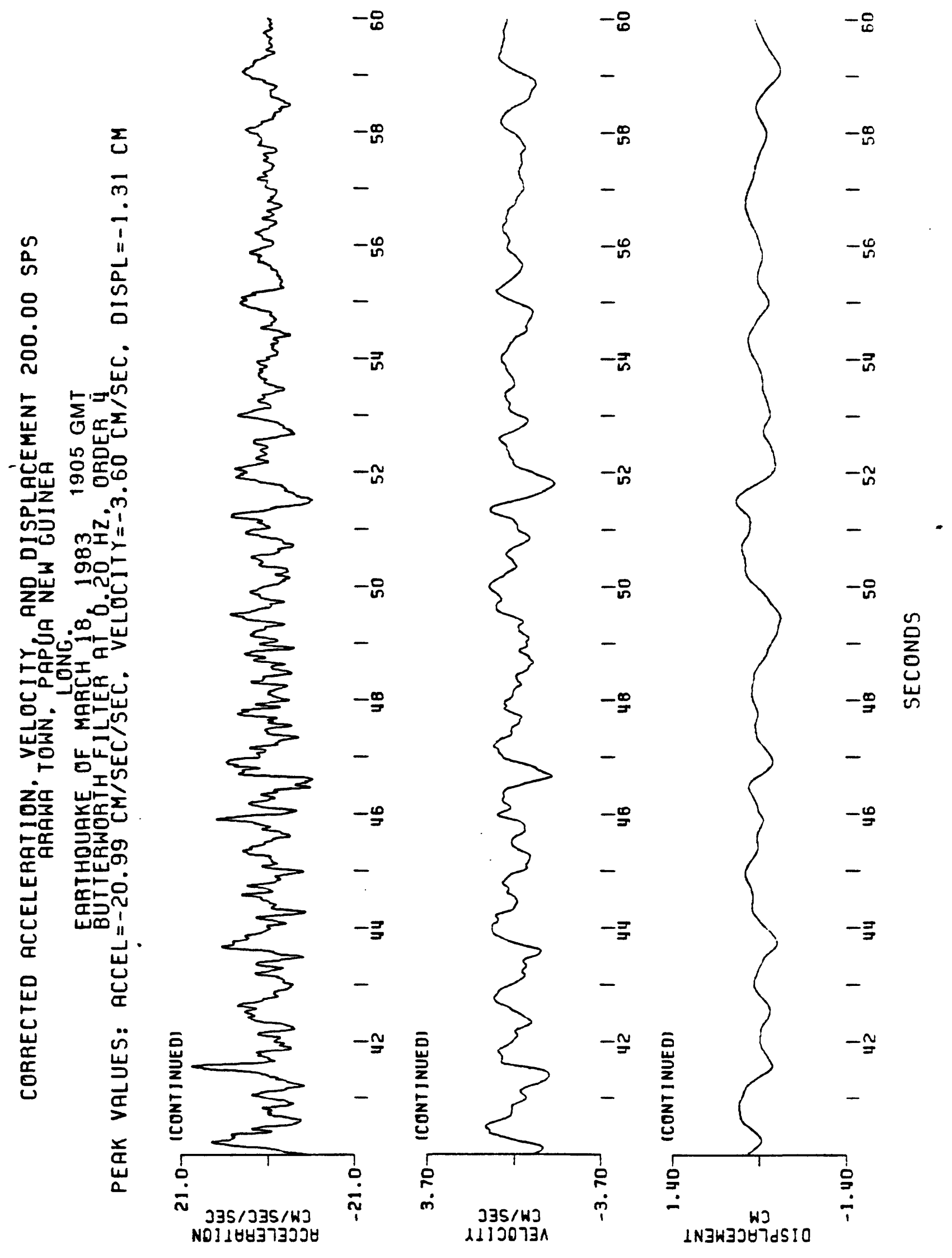




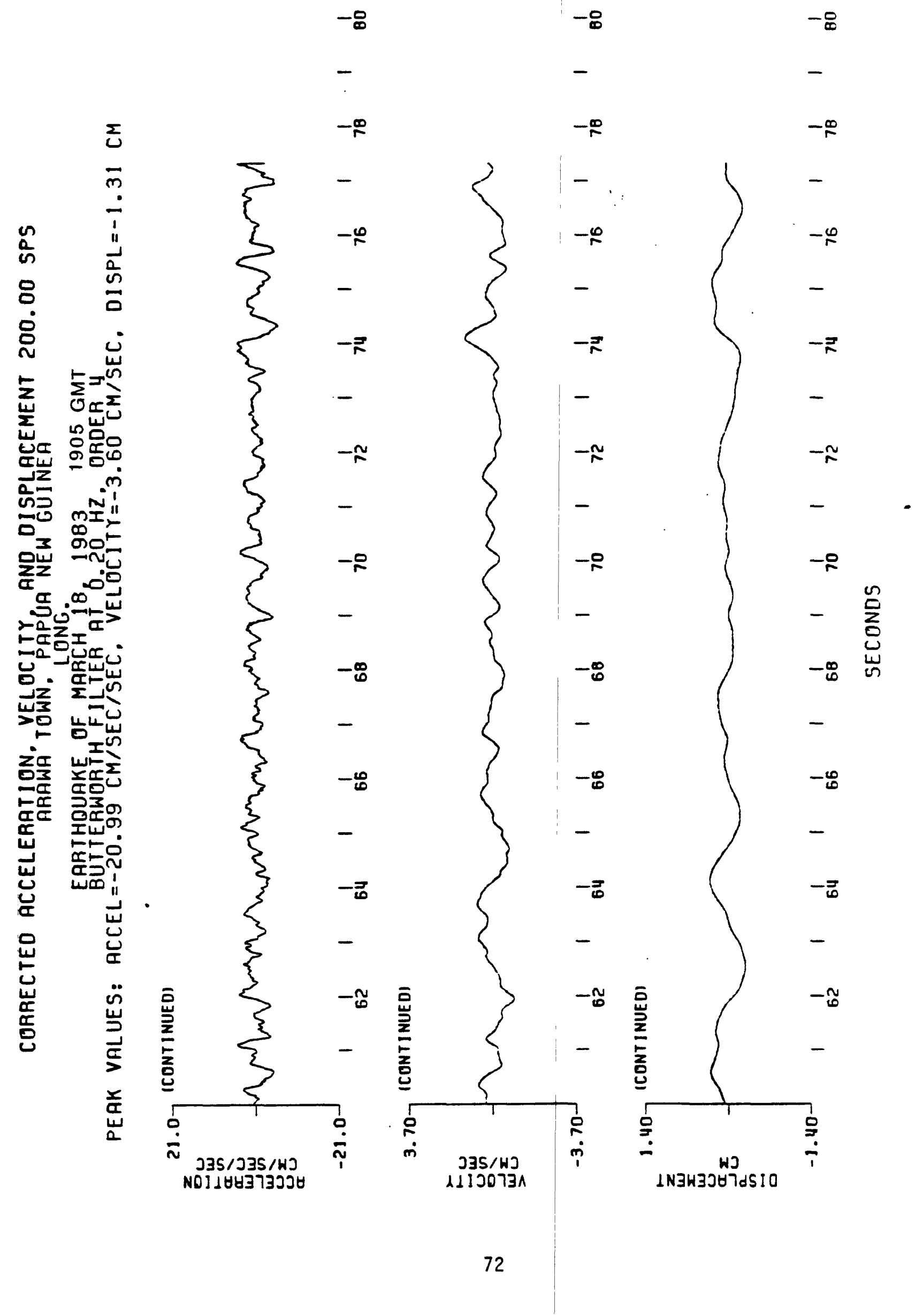




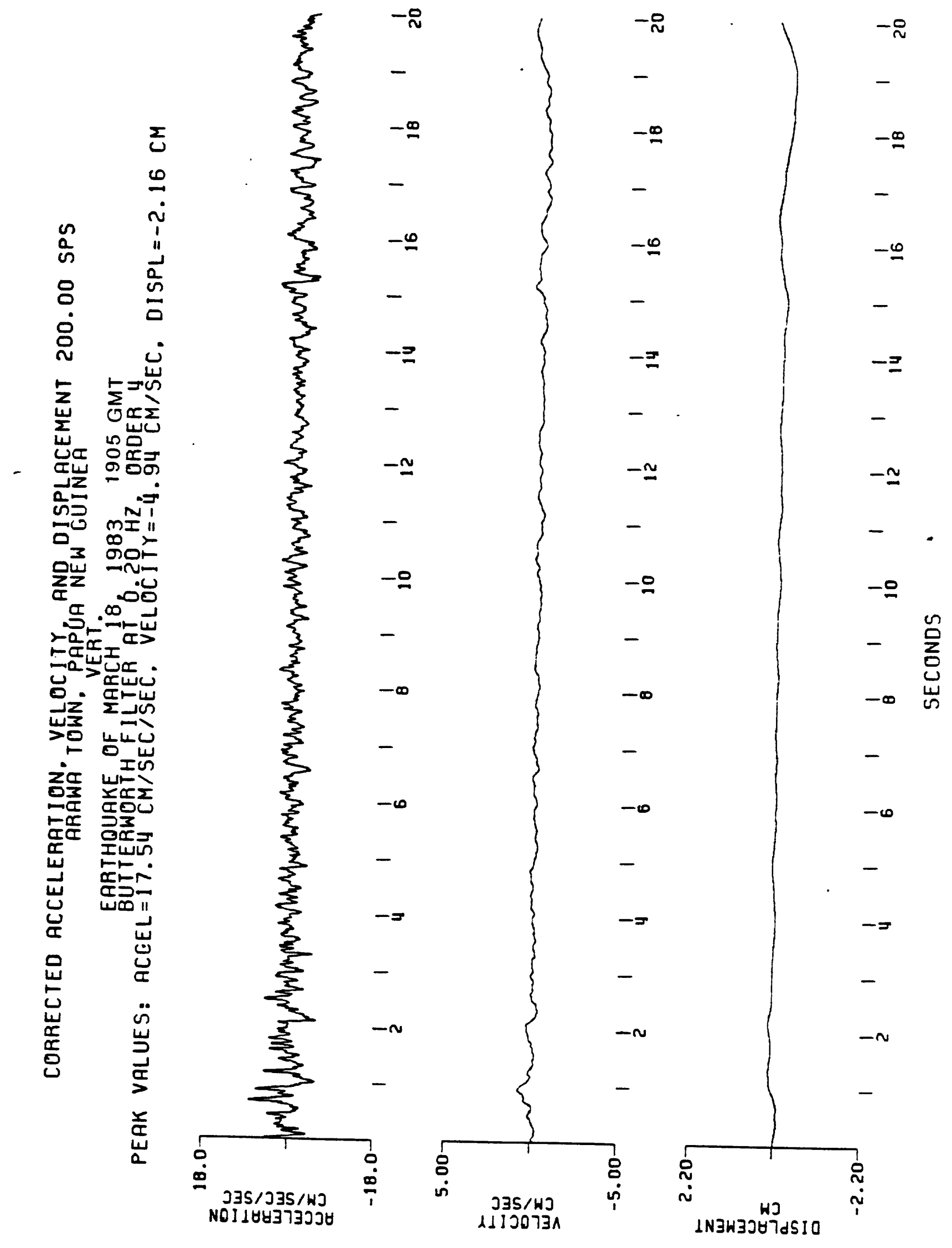




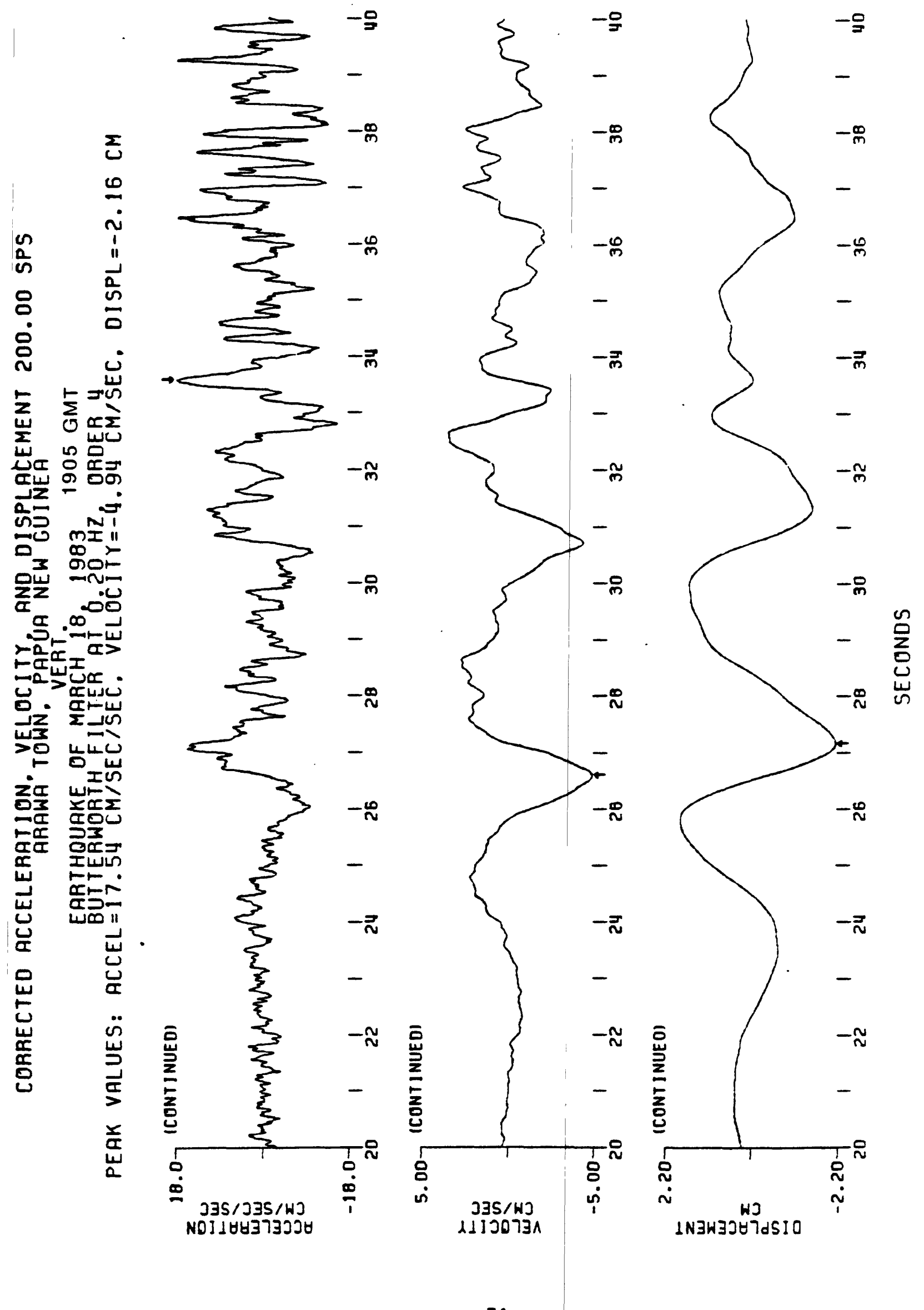




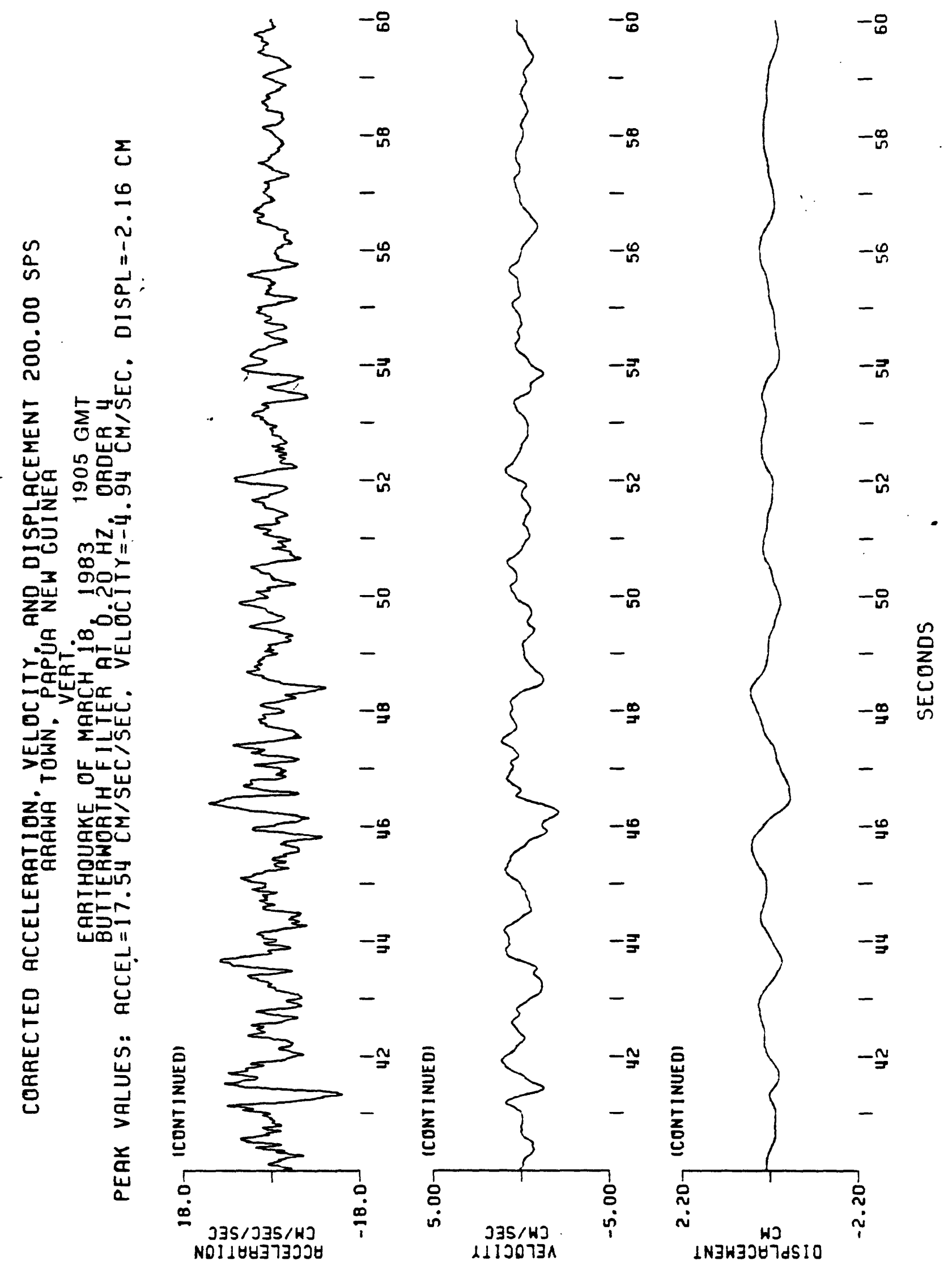




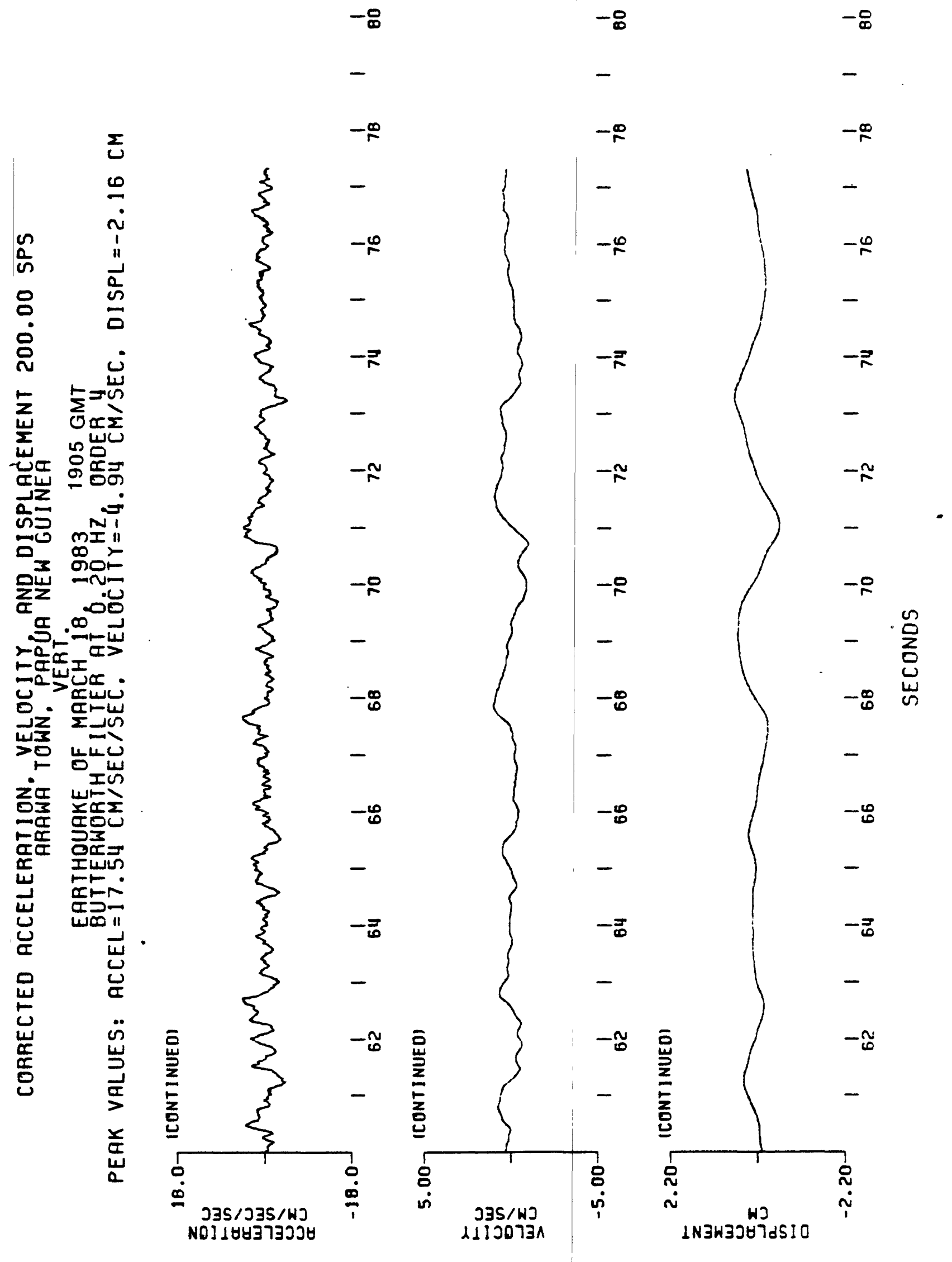




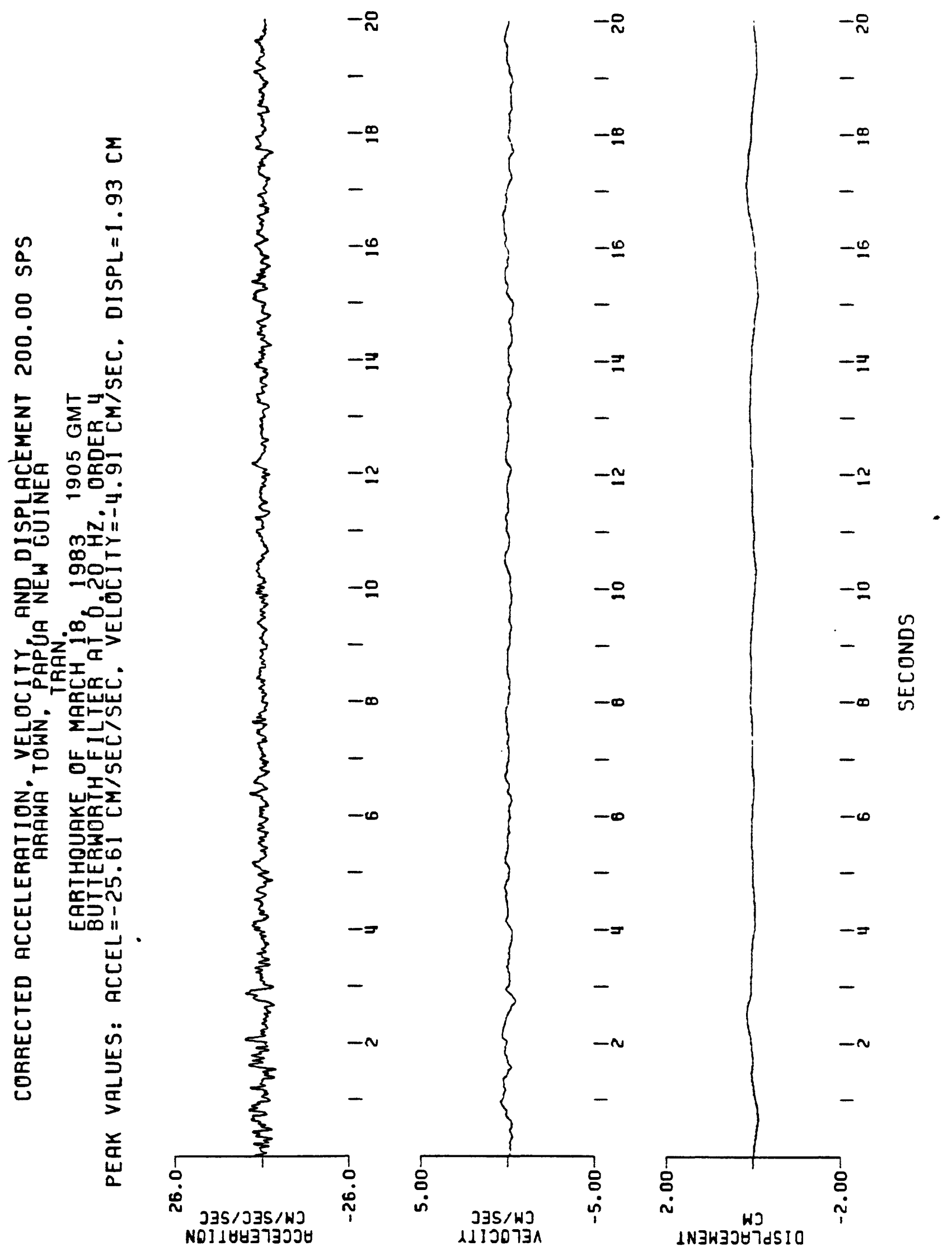




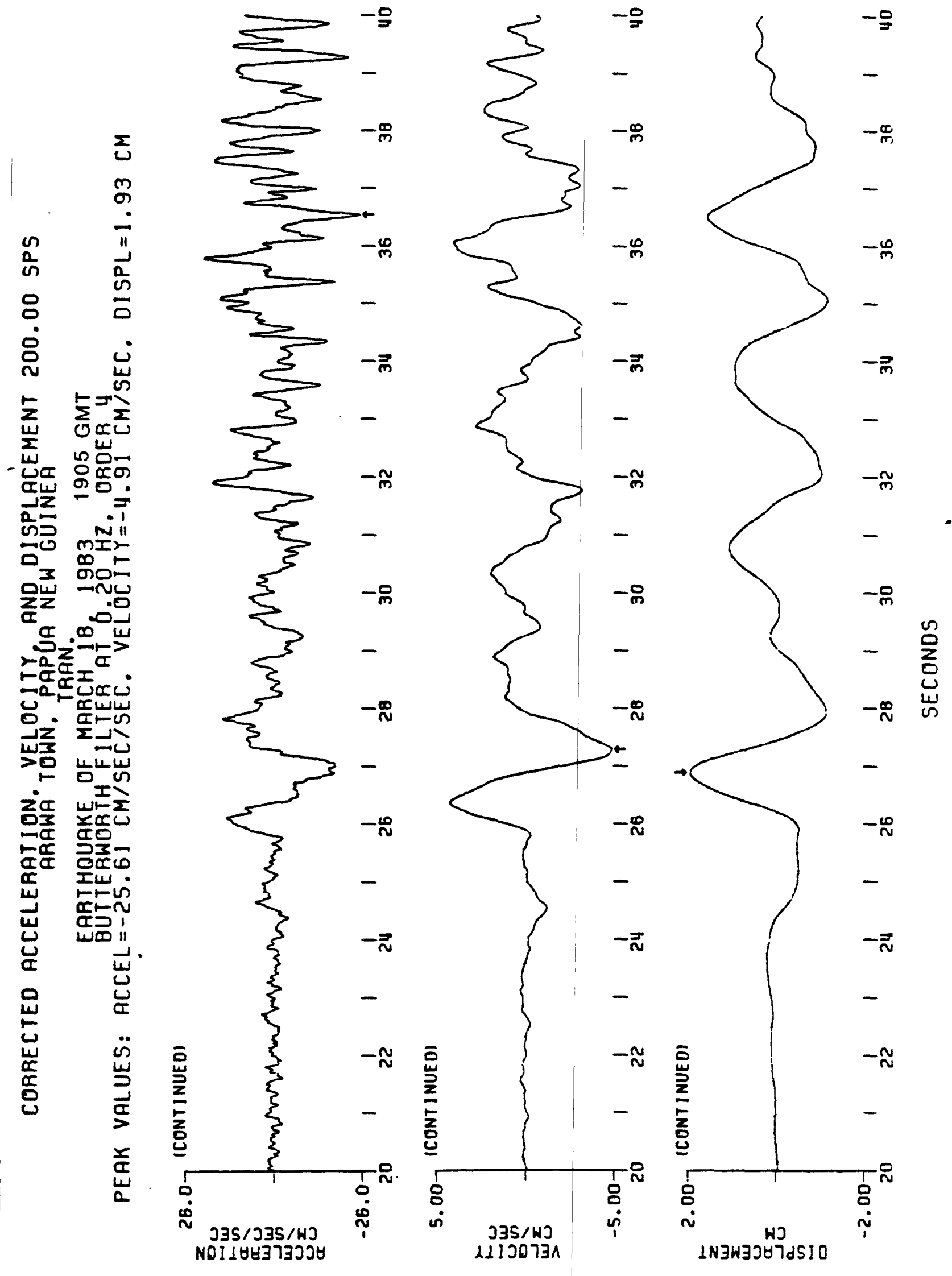




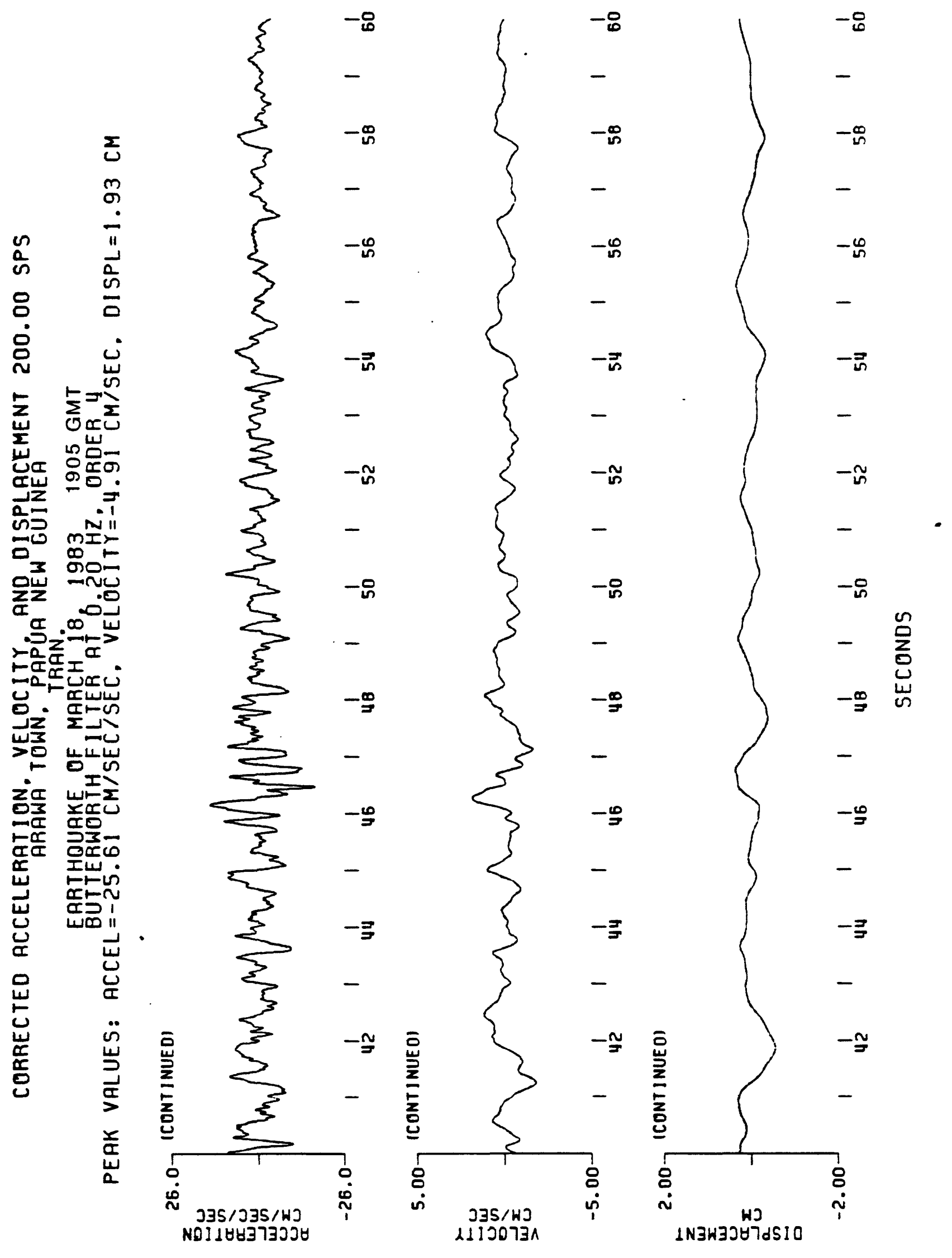




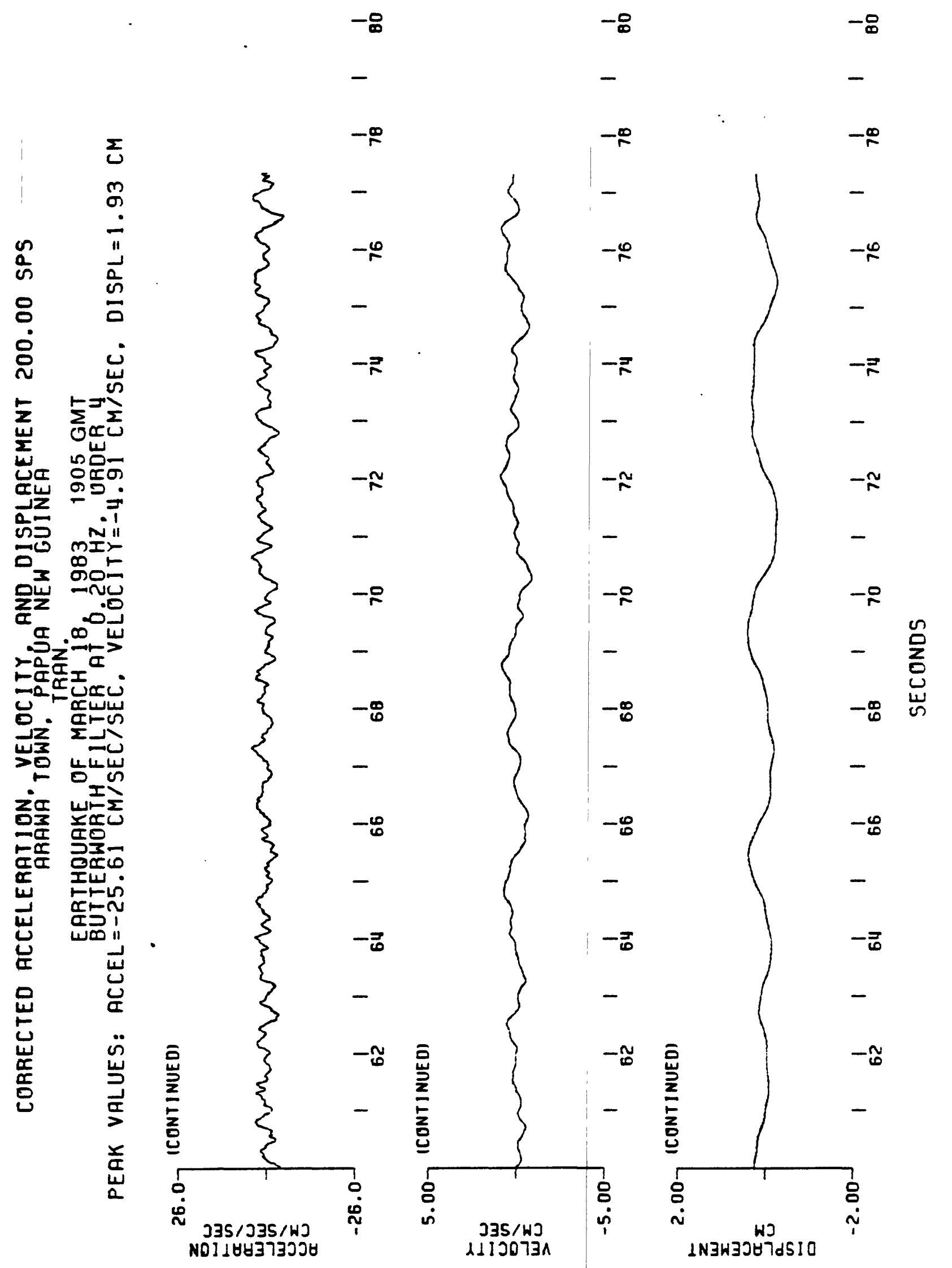




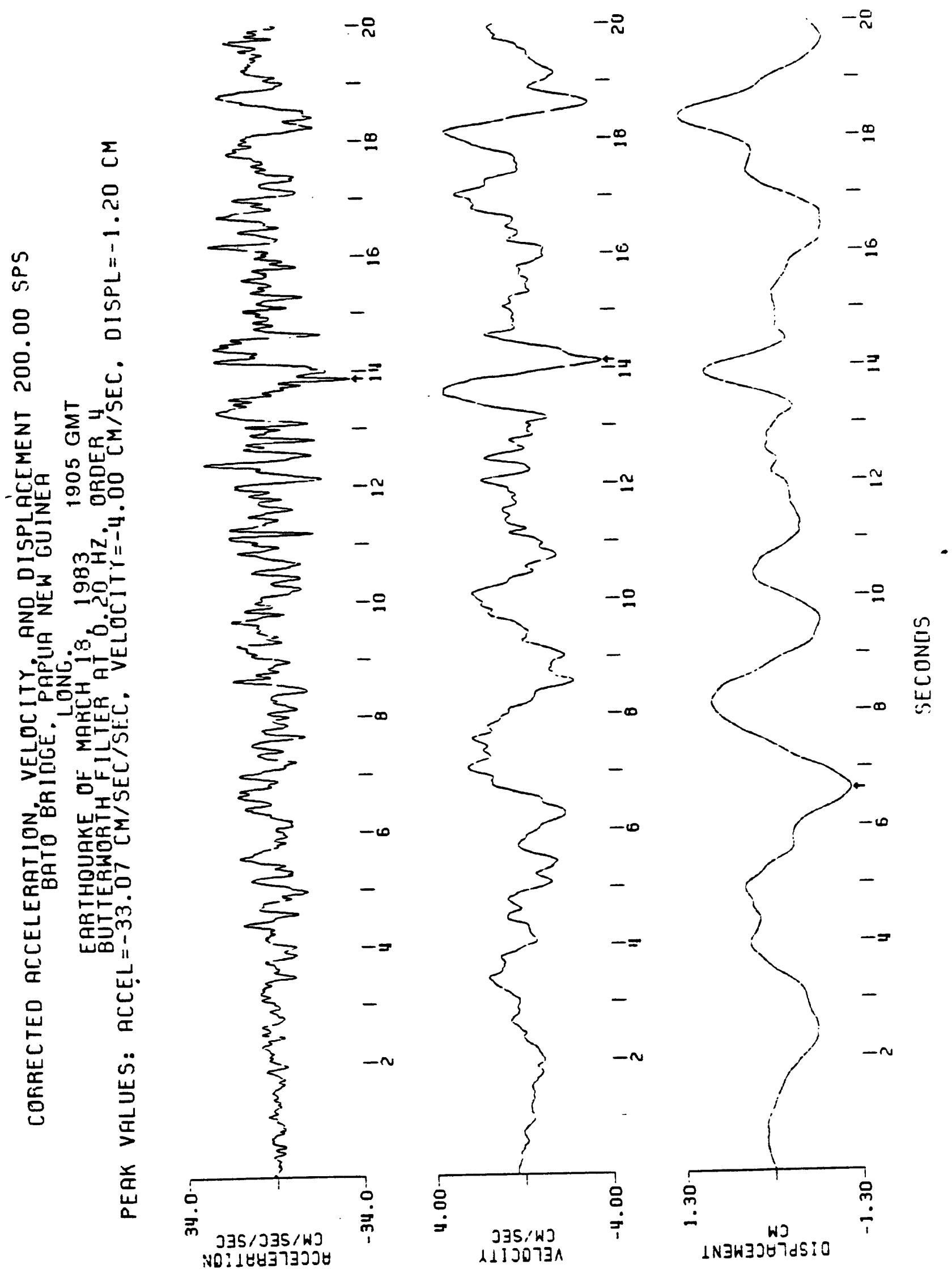




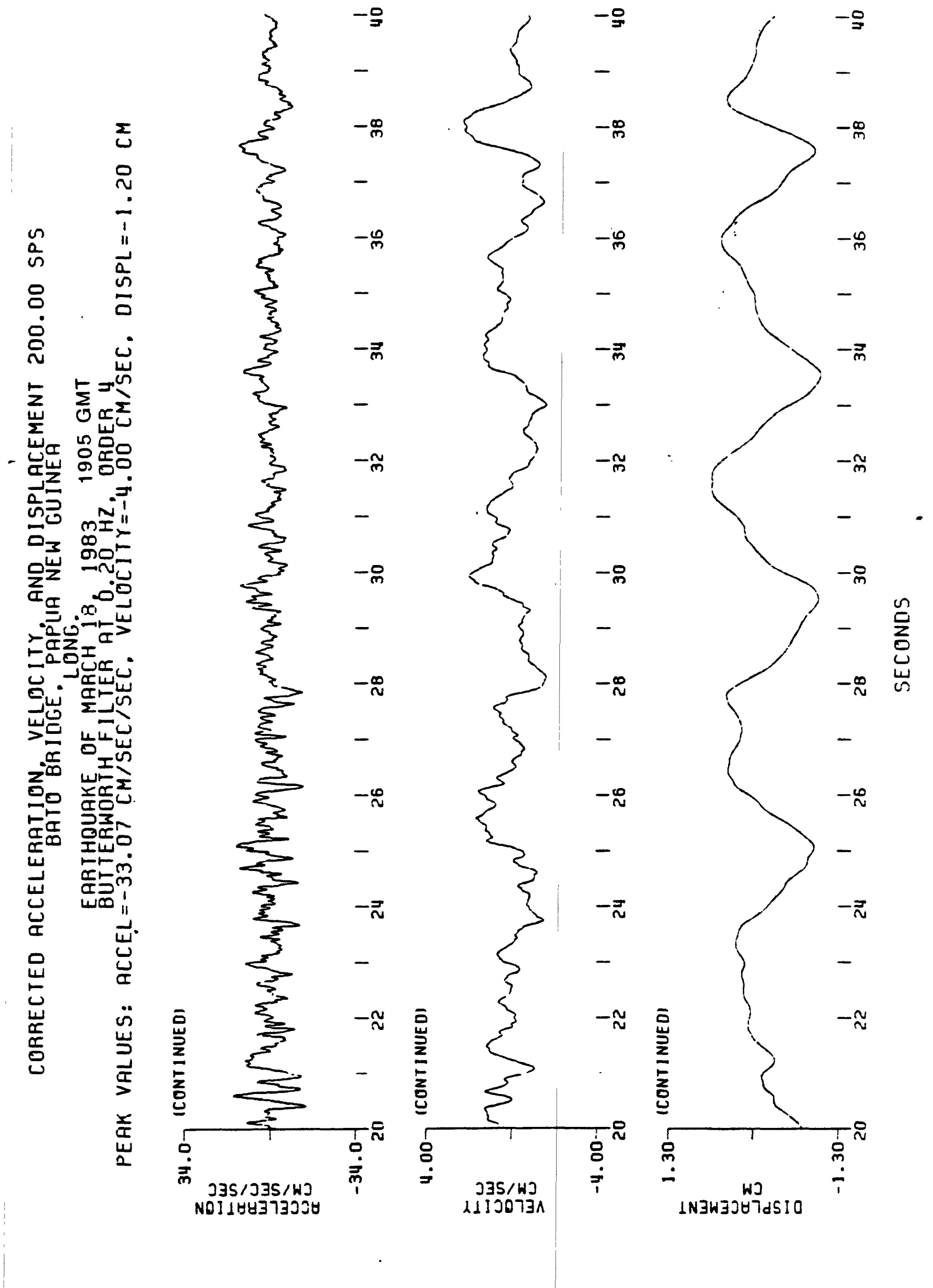




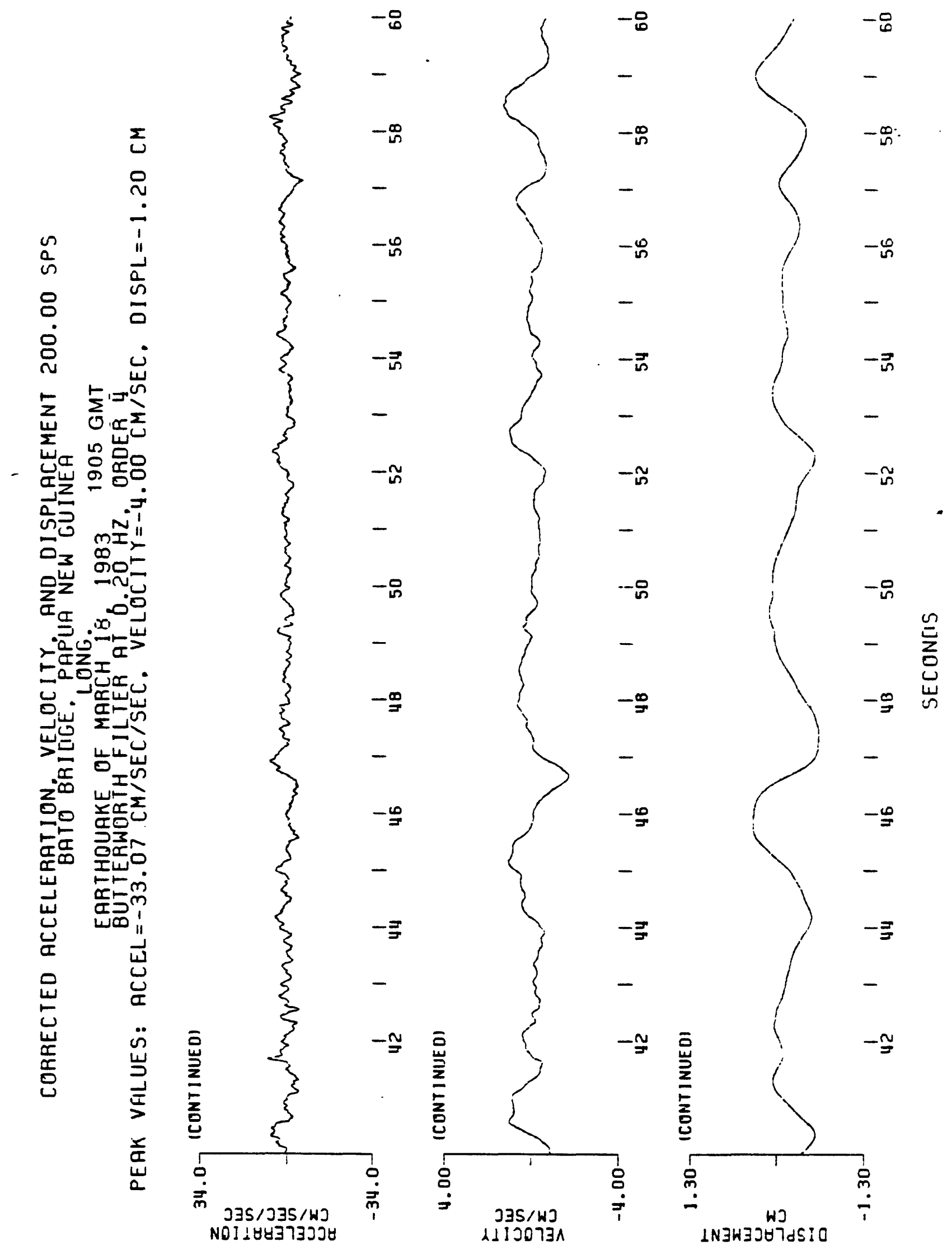




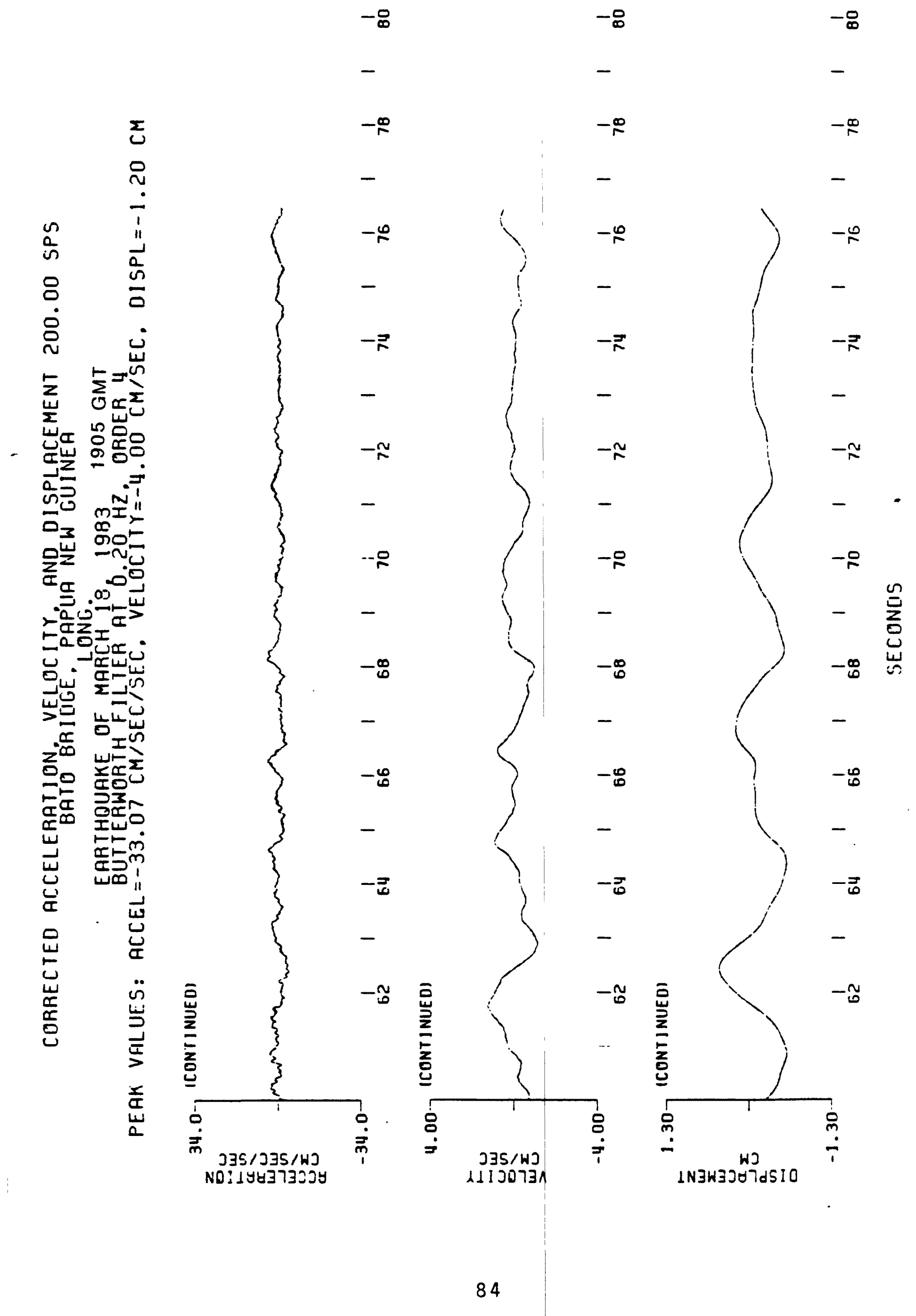




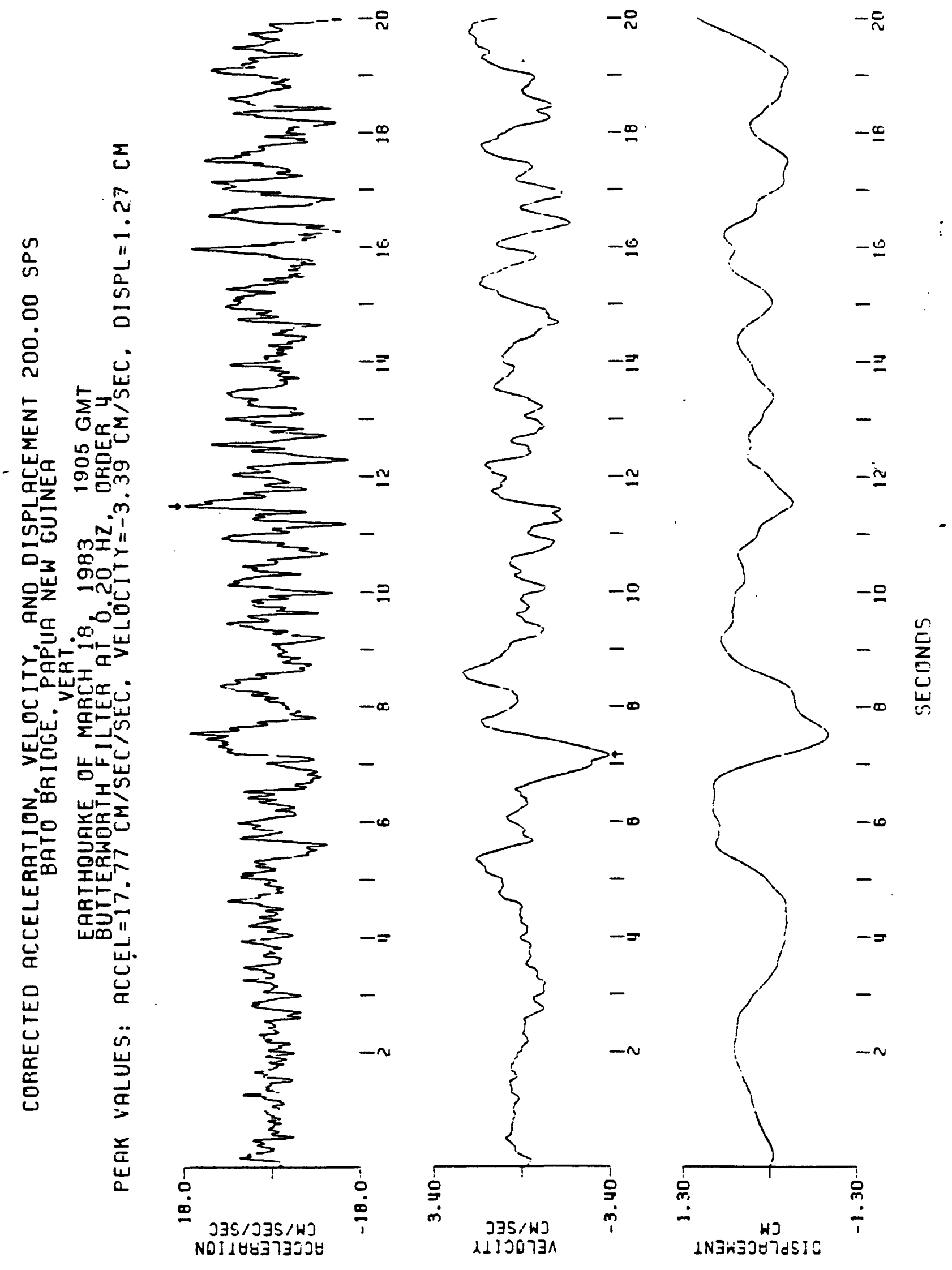




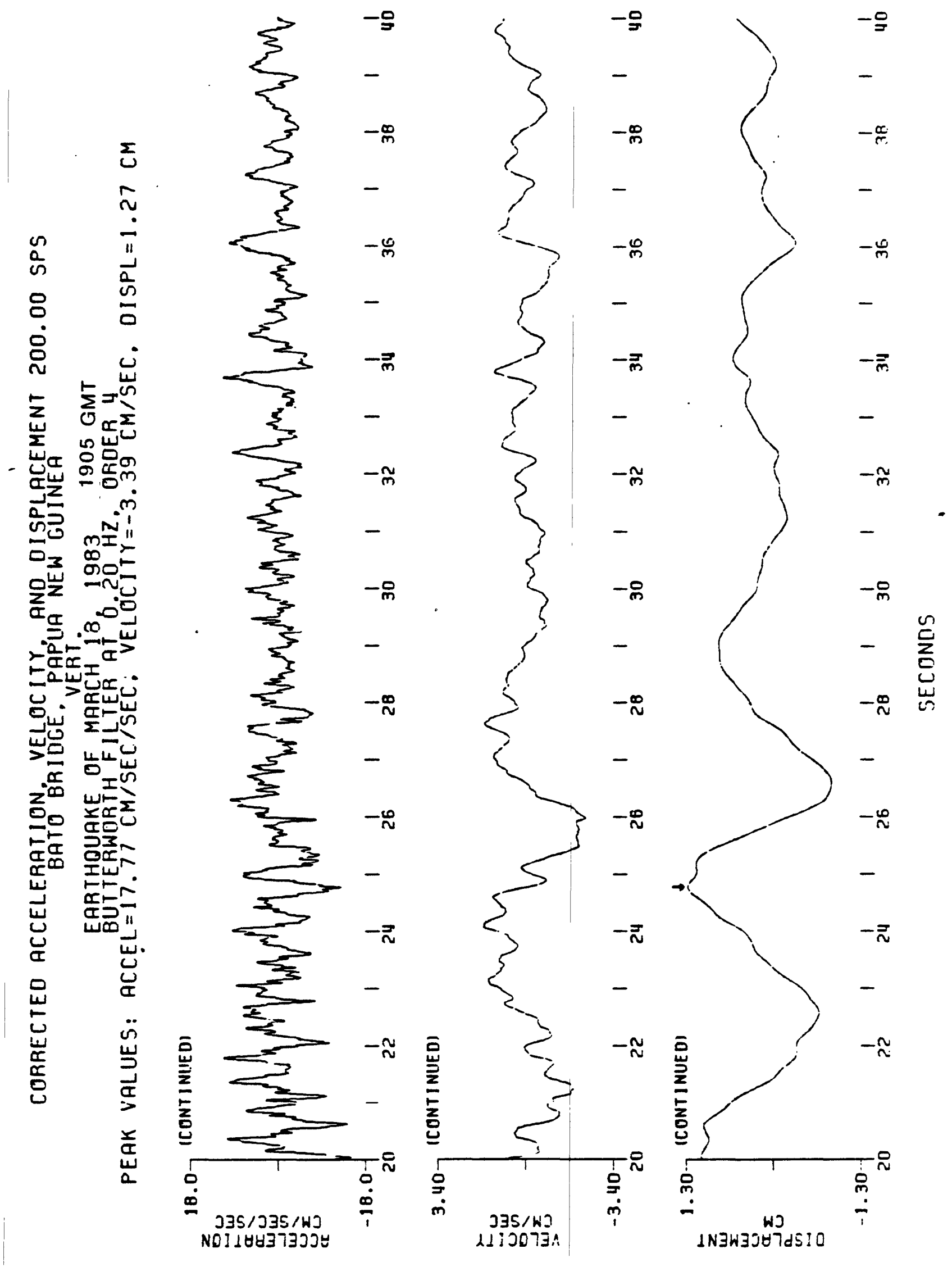




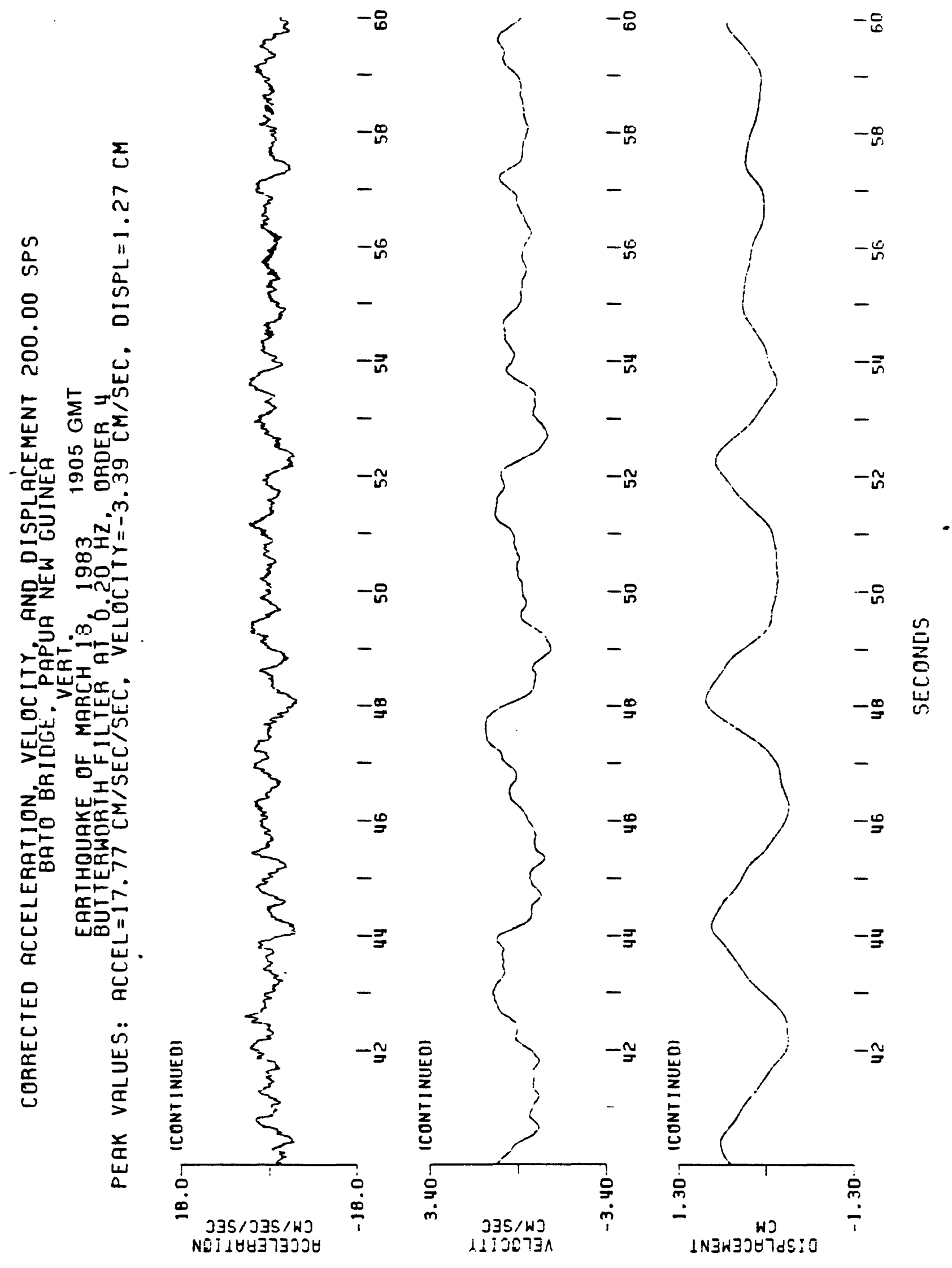




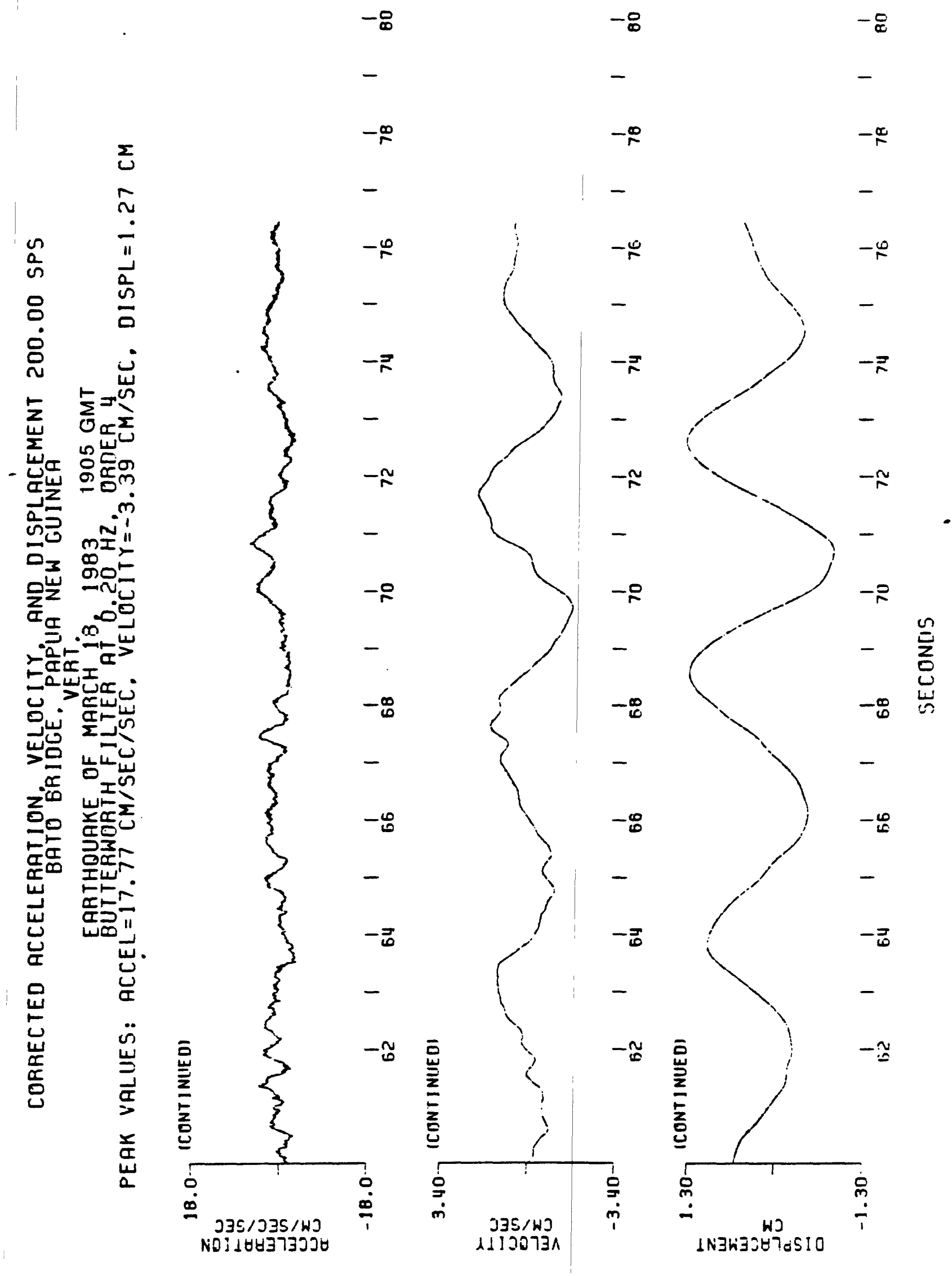




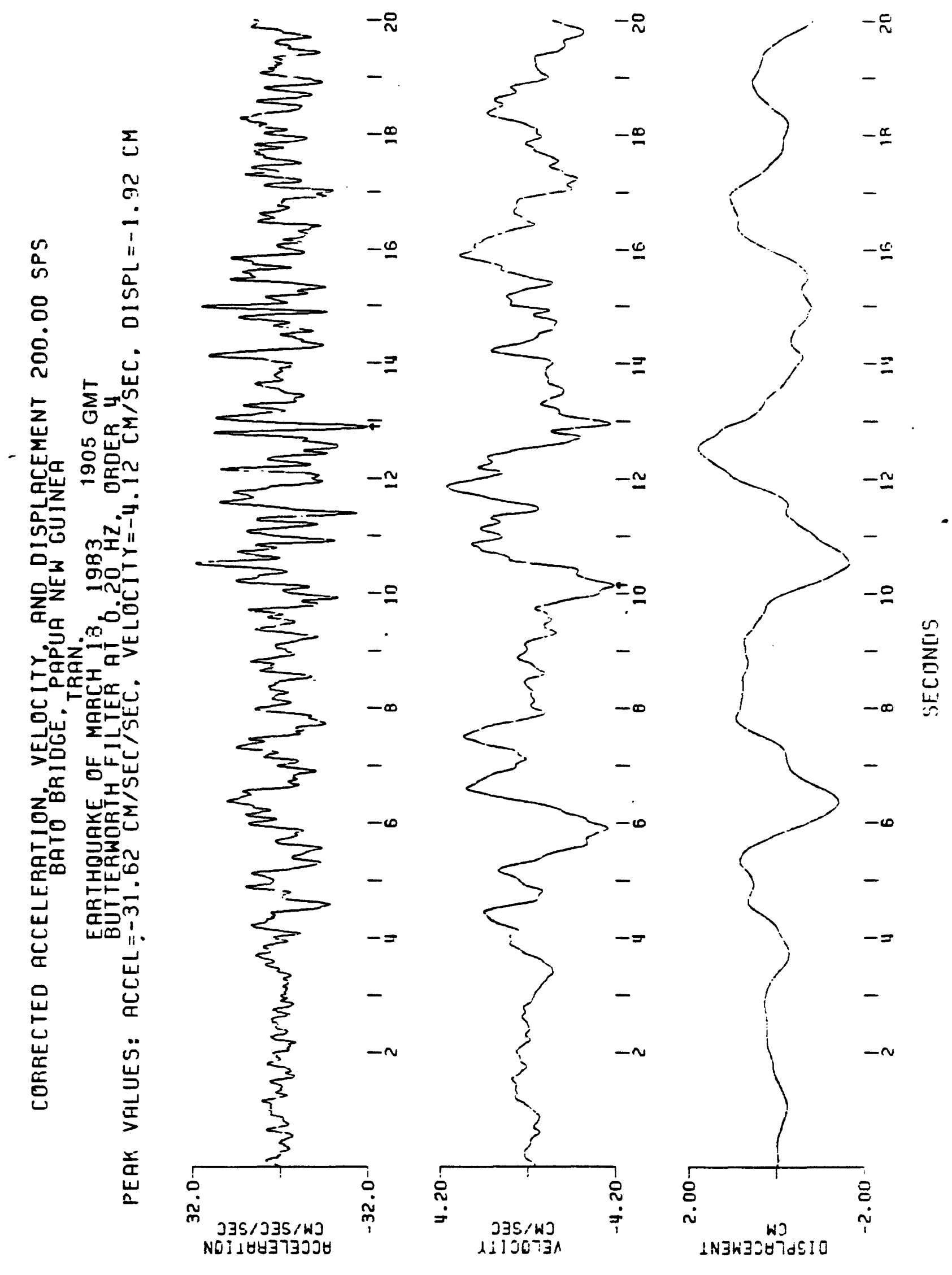




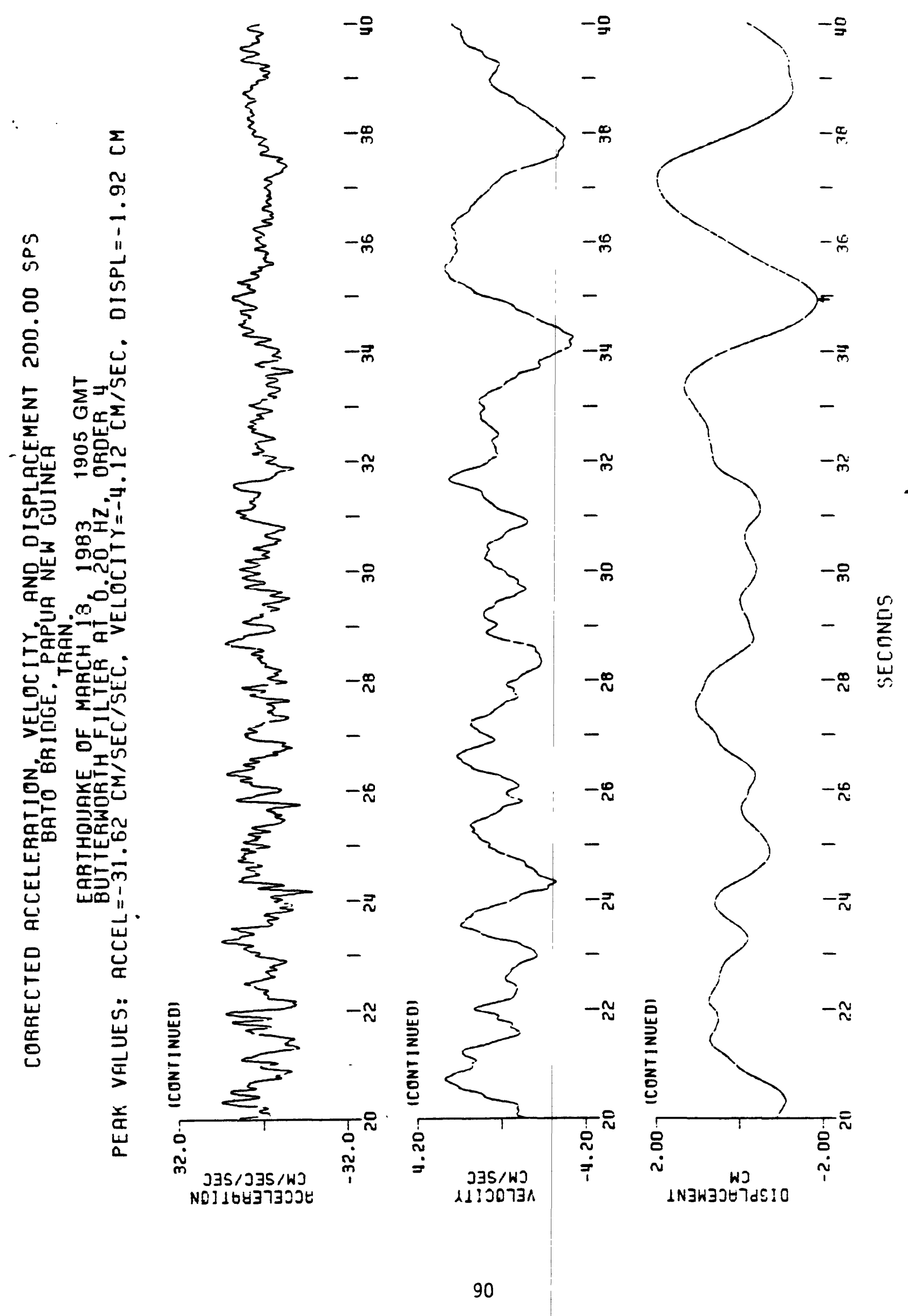




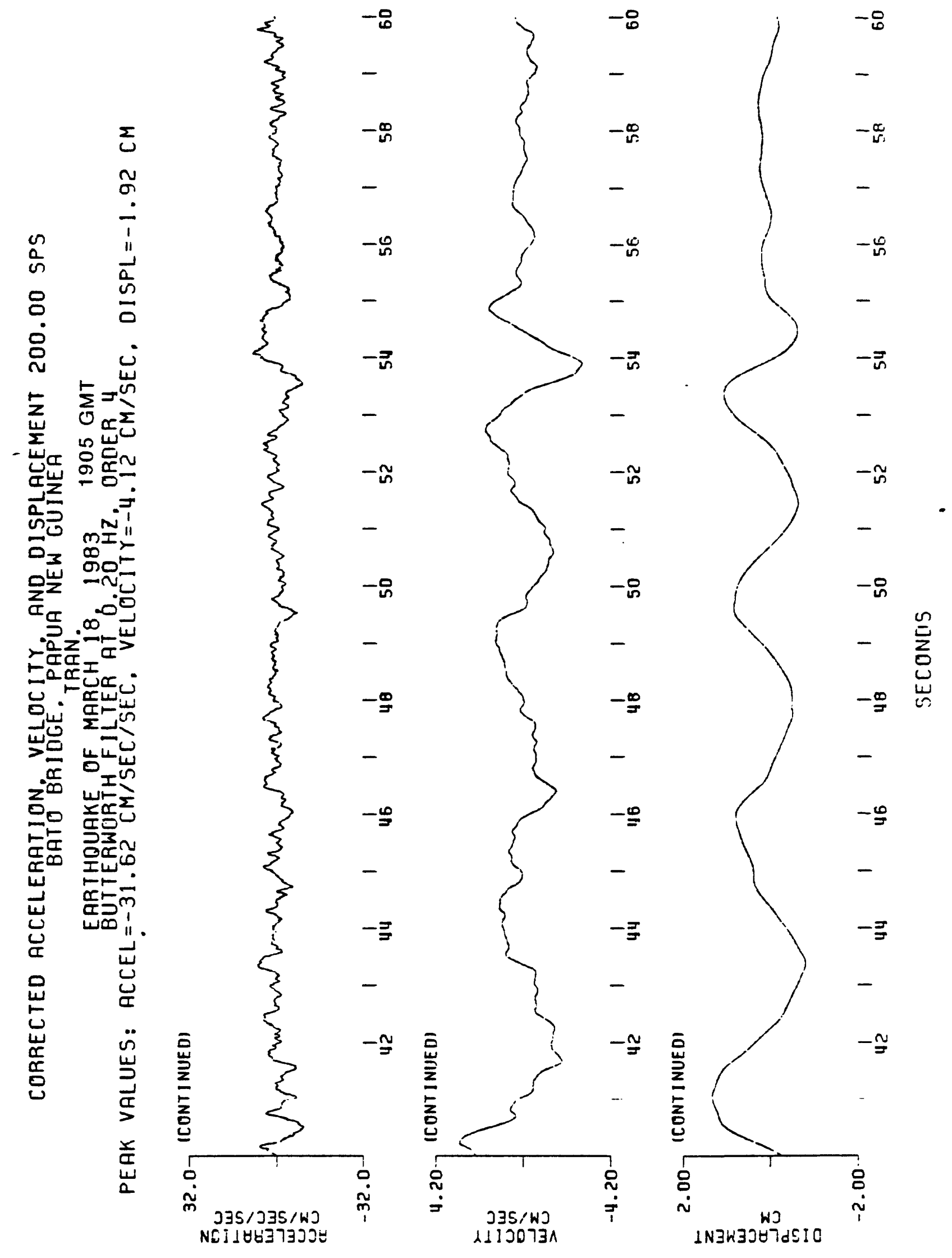




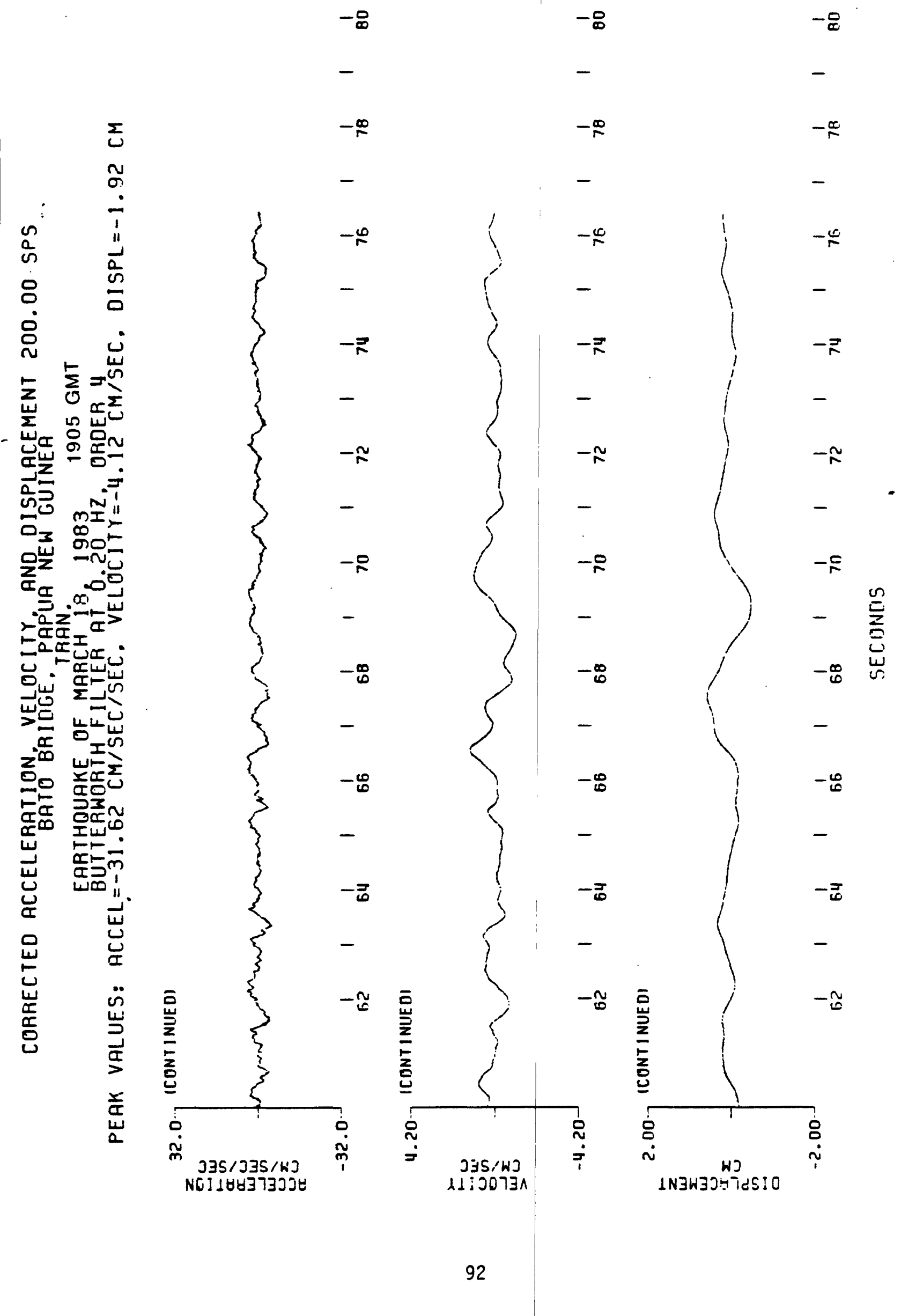




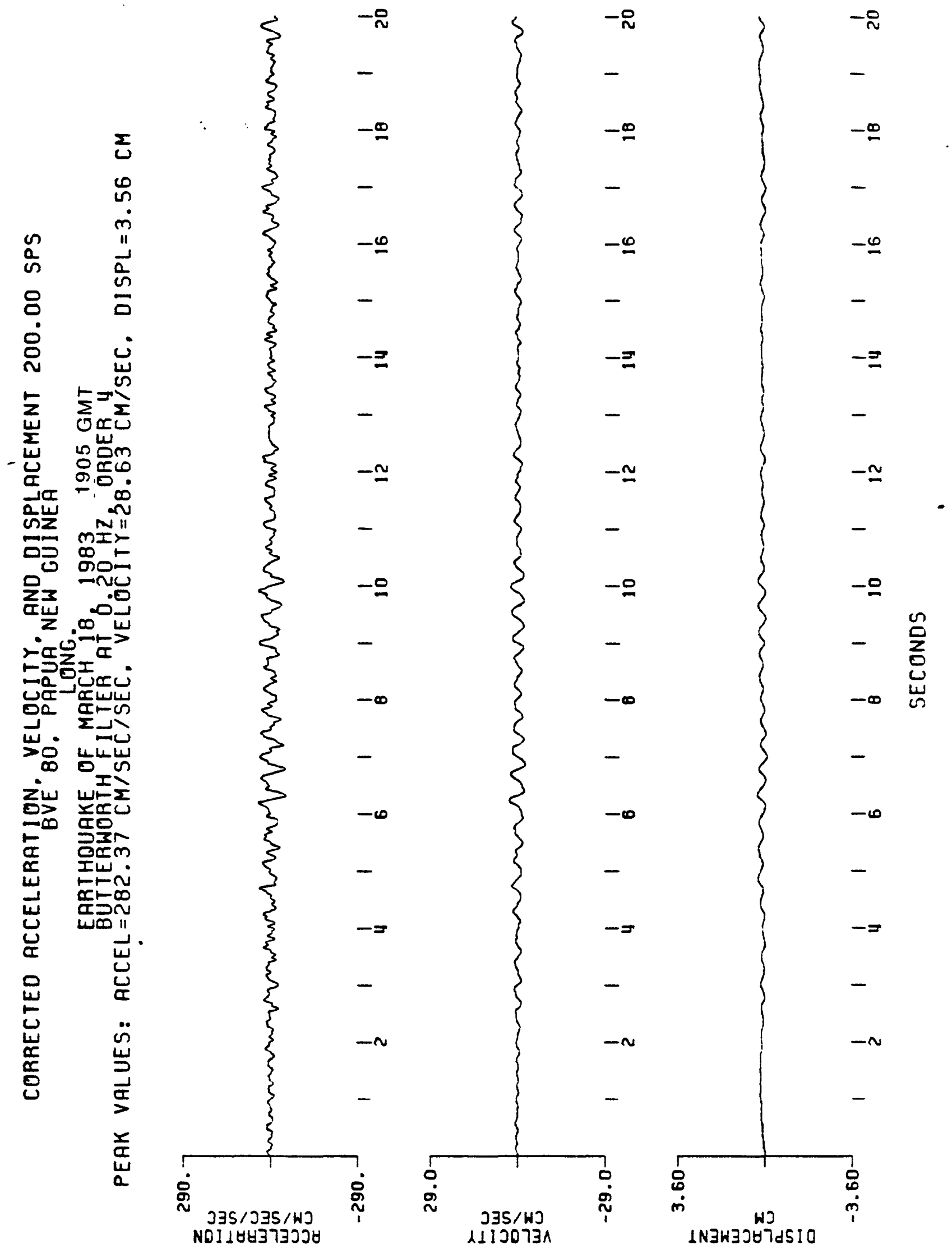




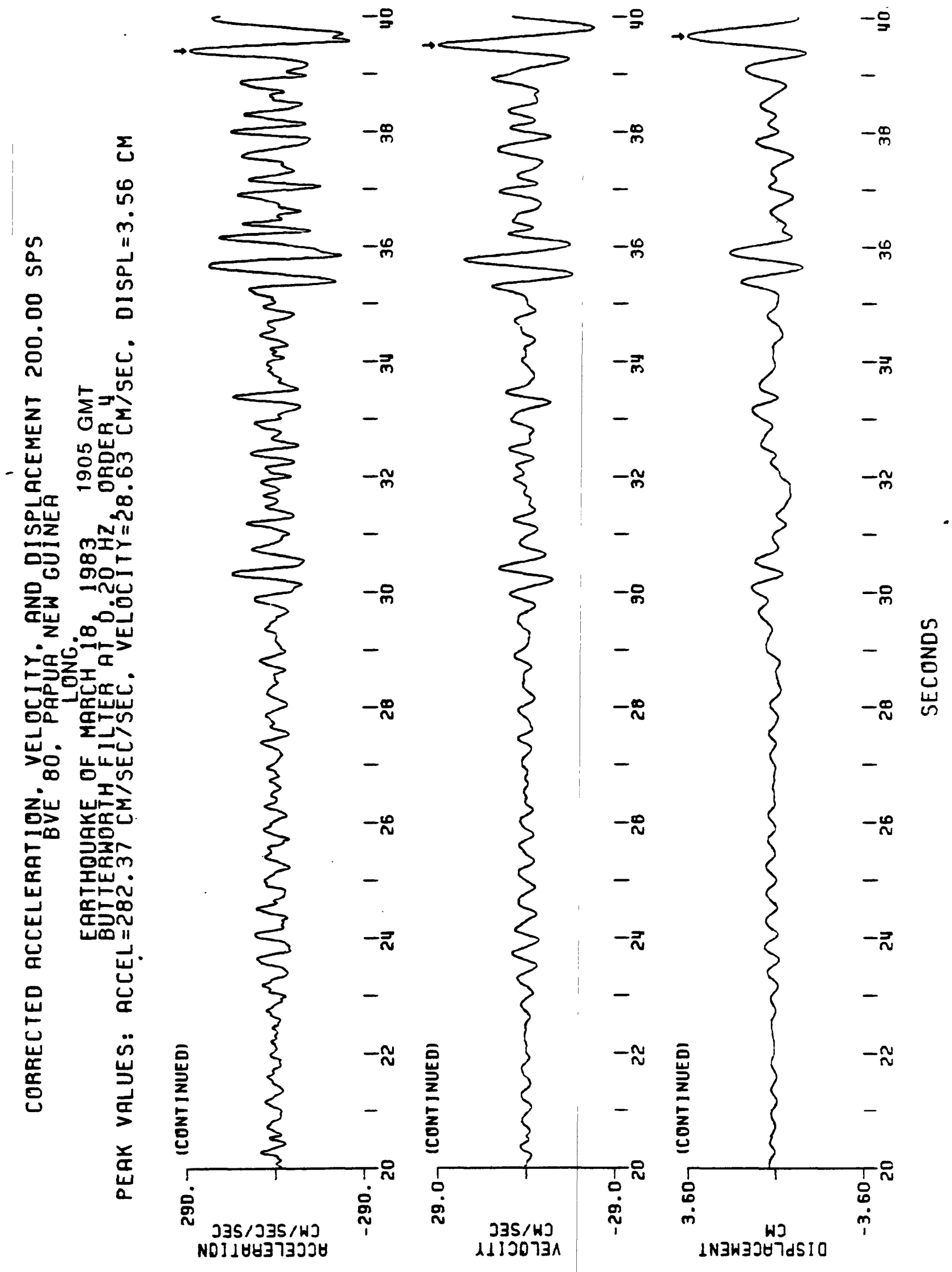




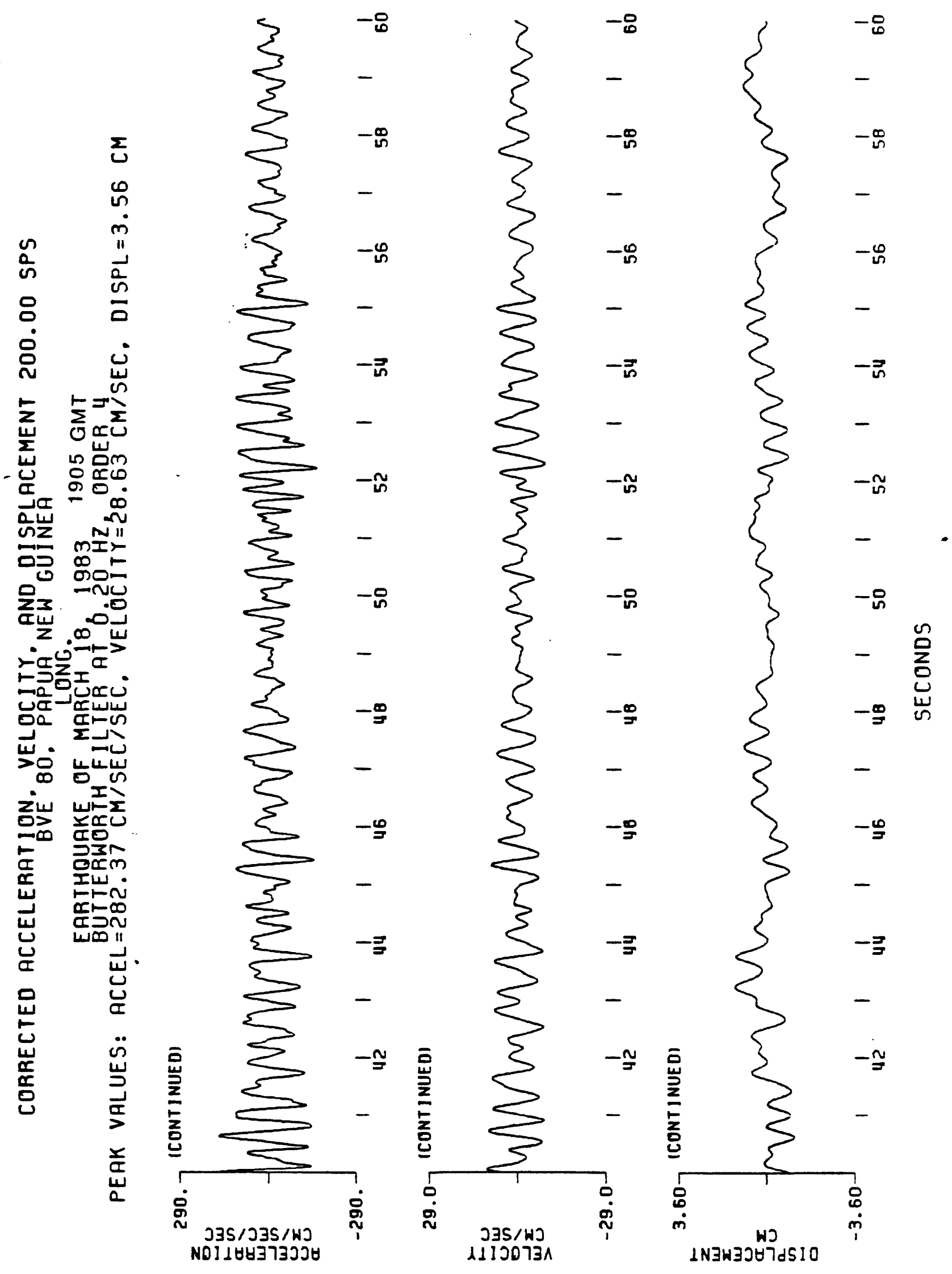




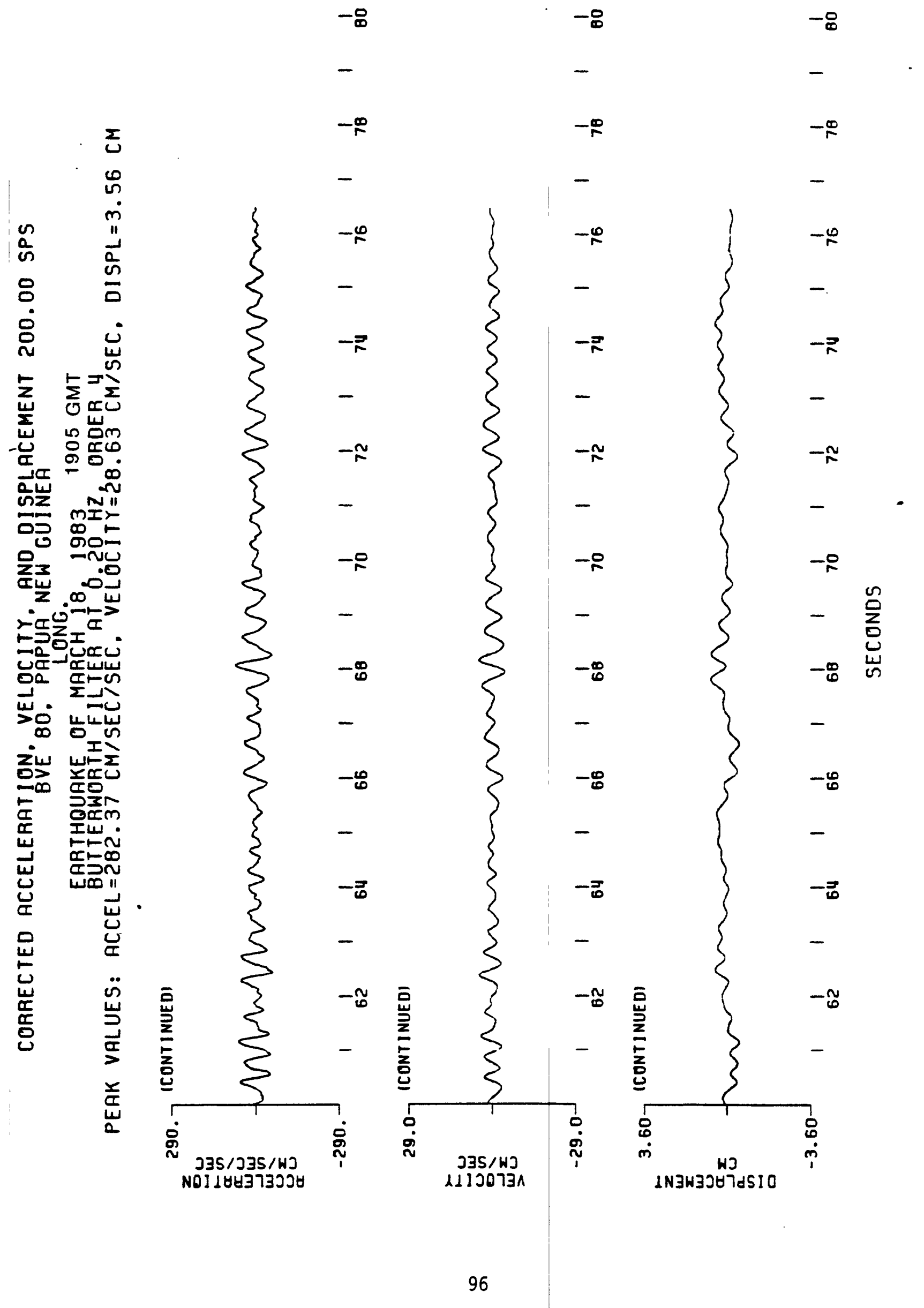




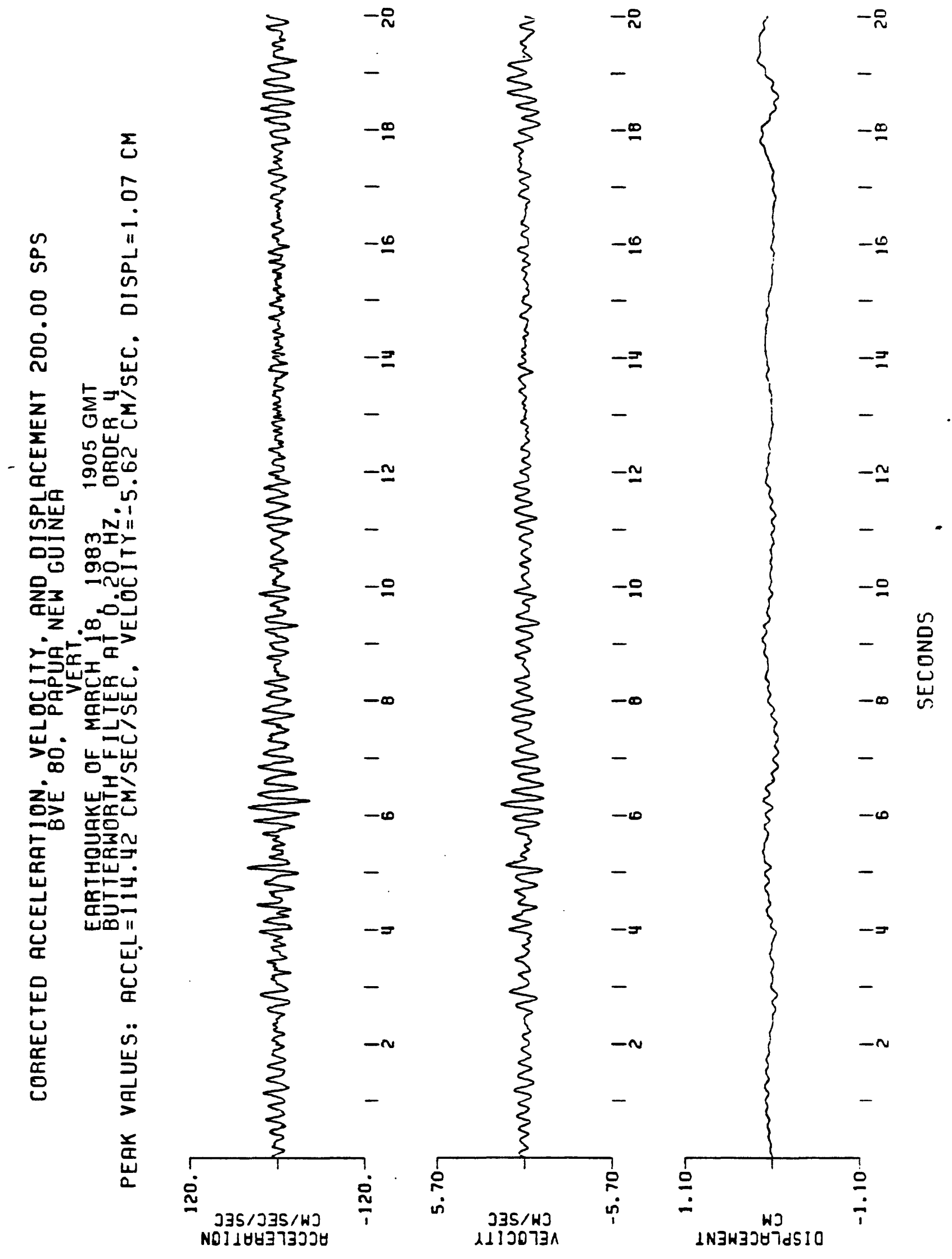




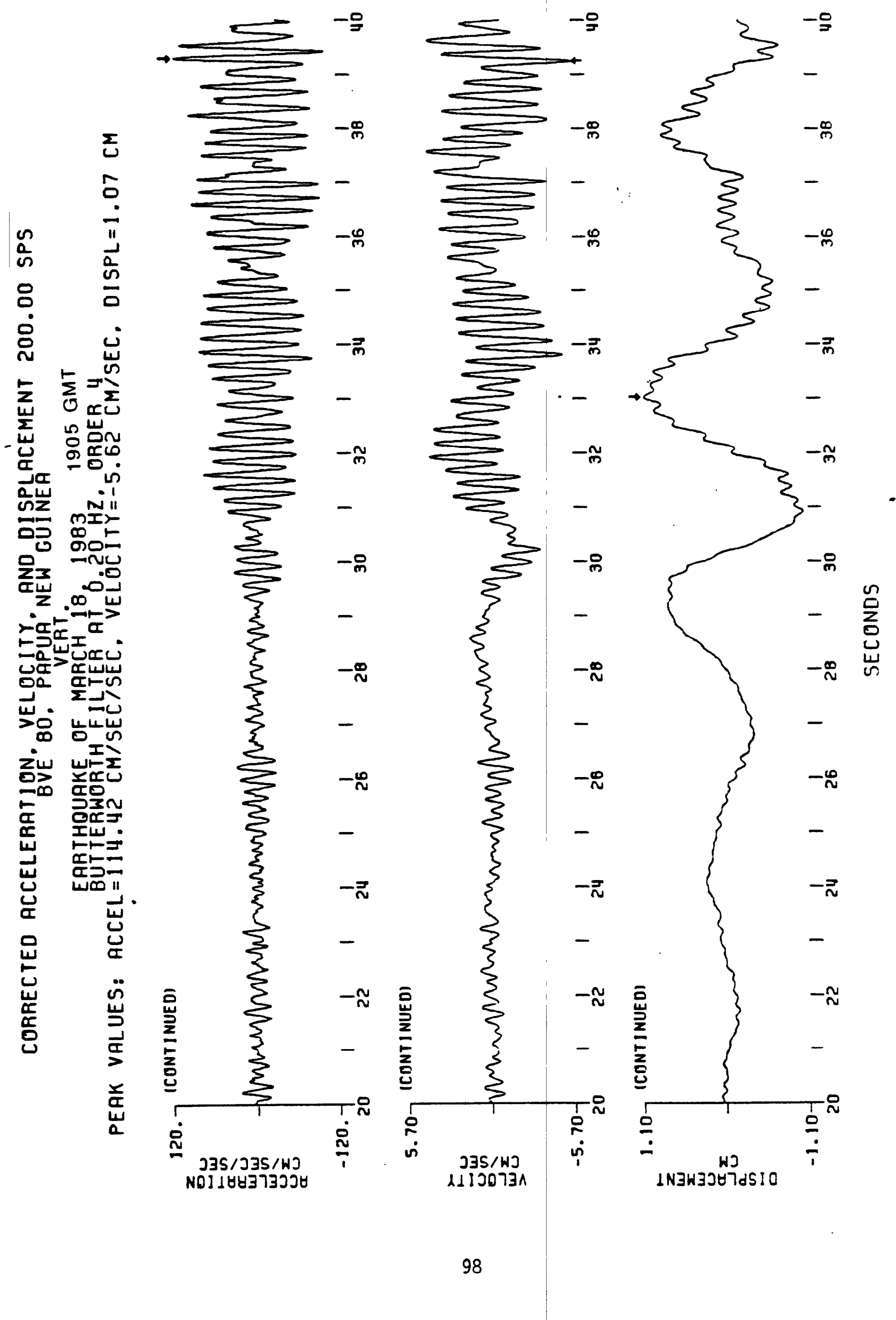




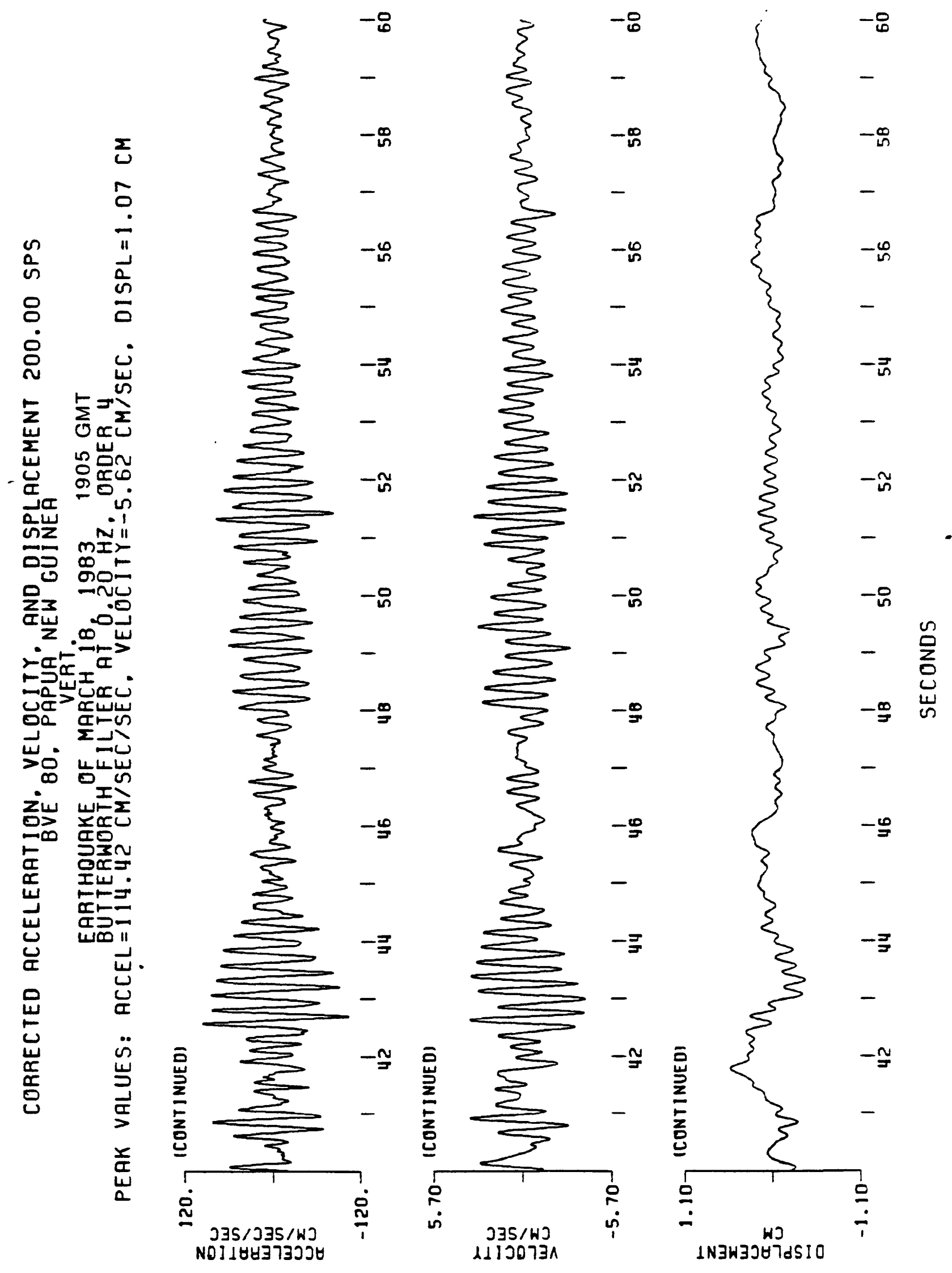




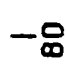

一囬

-

$\sum$

$-\infty$

$-\infty$

$-\frac{\infty}{r}$

ก

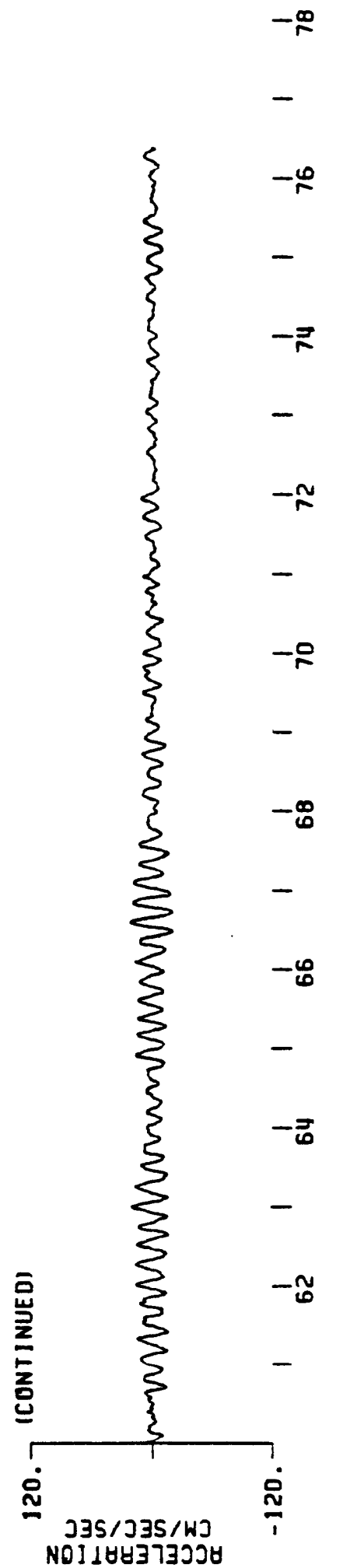

$\begin{array}{ll}\infty & \frac{1}{11} \\ 0 & \frac{1}{0}\end{array}$

只

i

岁

to

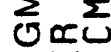

u

n

동

पa

व्य

जi NII

ज马 क्र

只崖

$\therefore-\frac{\infty}{a}$

-5先I

ن高>

Da वाw

w. $\quad \Sigma=0$

$>0$ 吕出

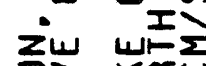

닝

$\underbrace{\infty}$ 독옹

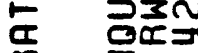

采 至山。

뜨믐

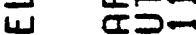

U 山क几

의

임
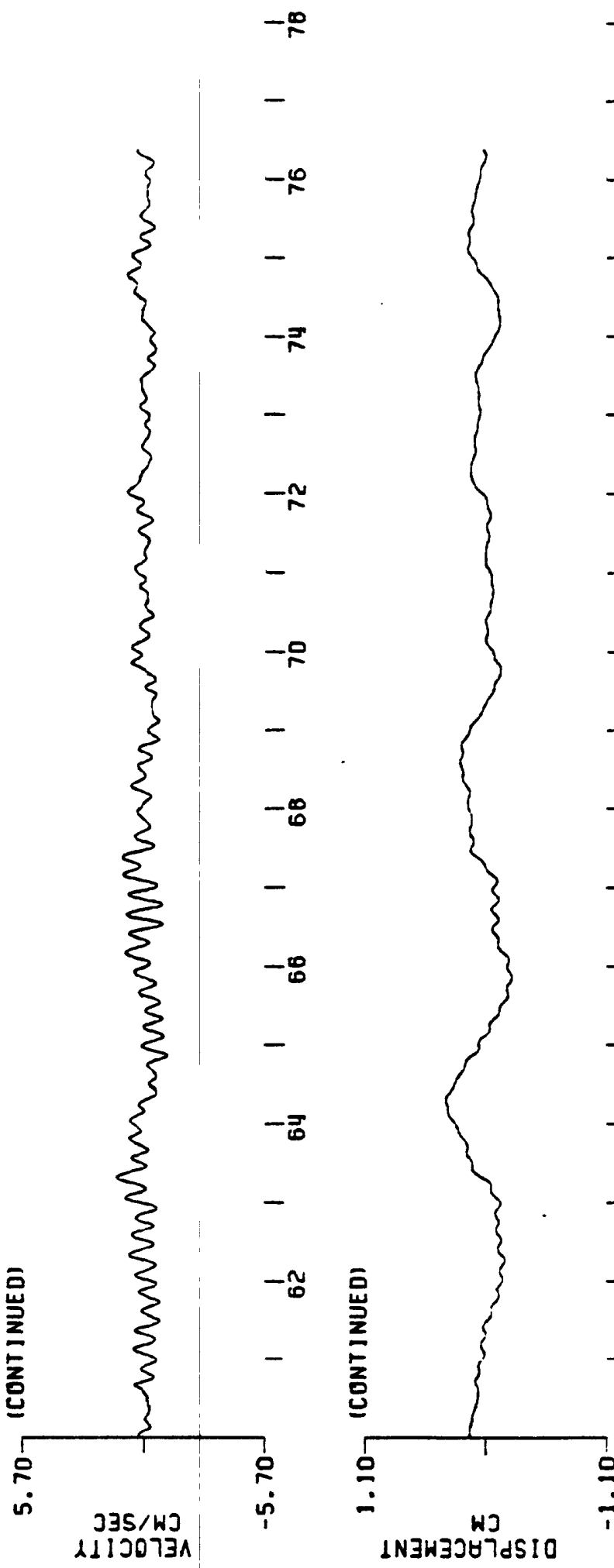

㭊

ته

秃

No1 $14837 \exists 354$

$110073 \wedge$

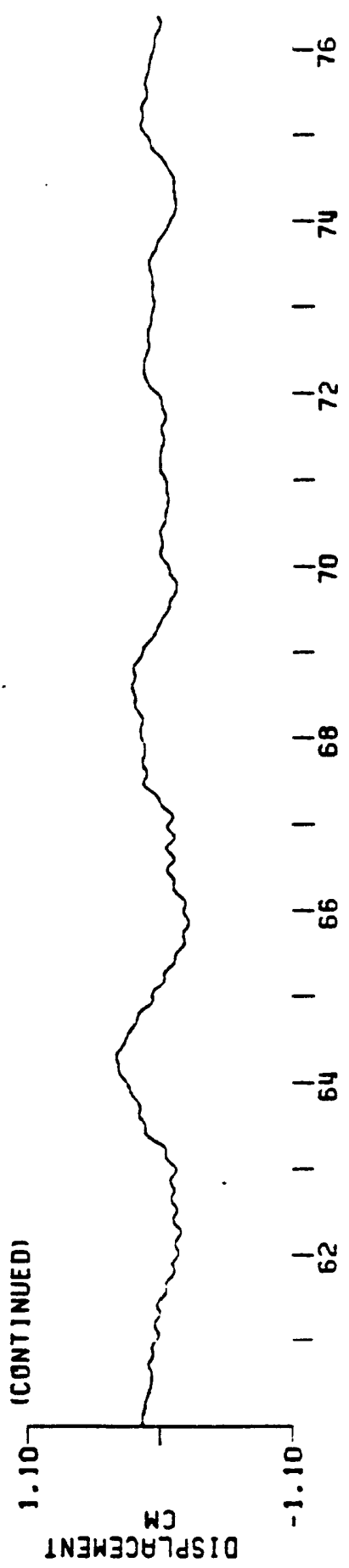




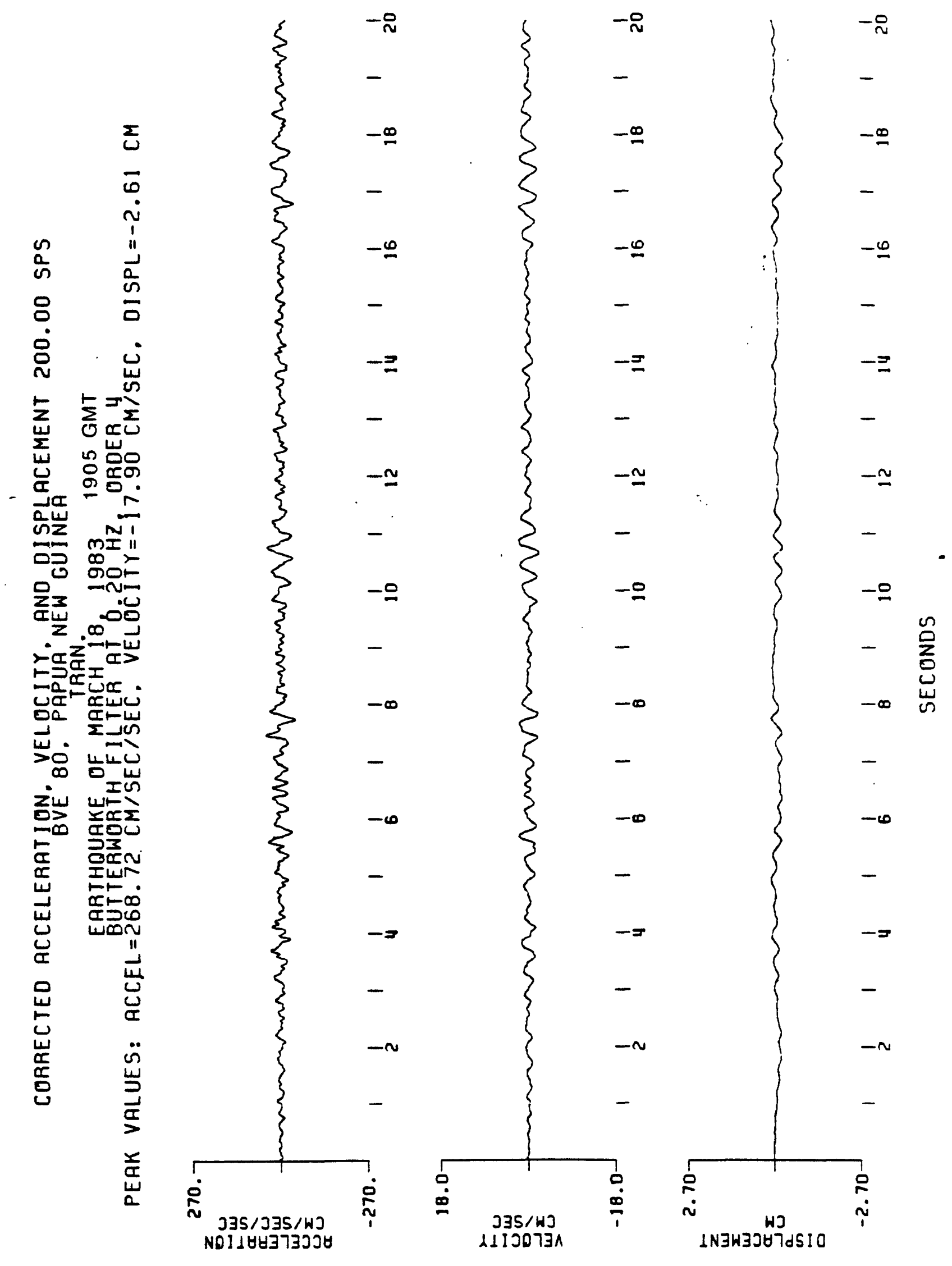




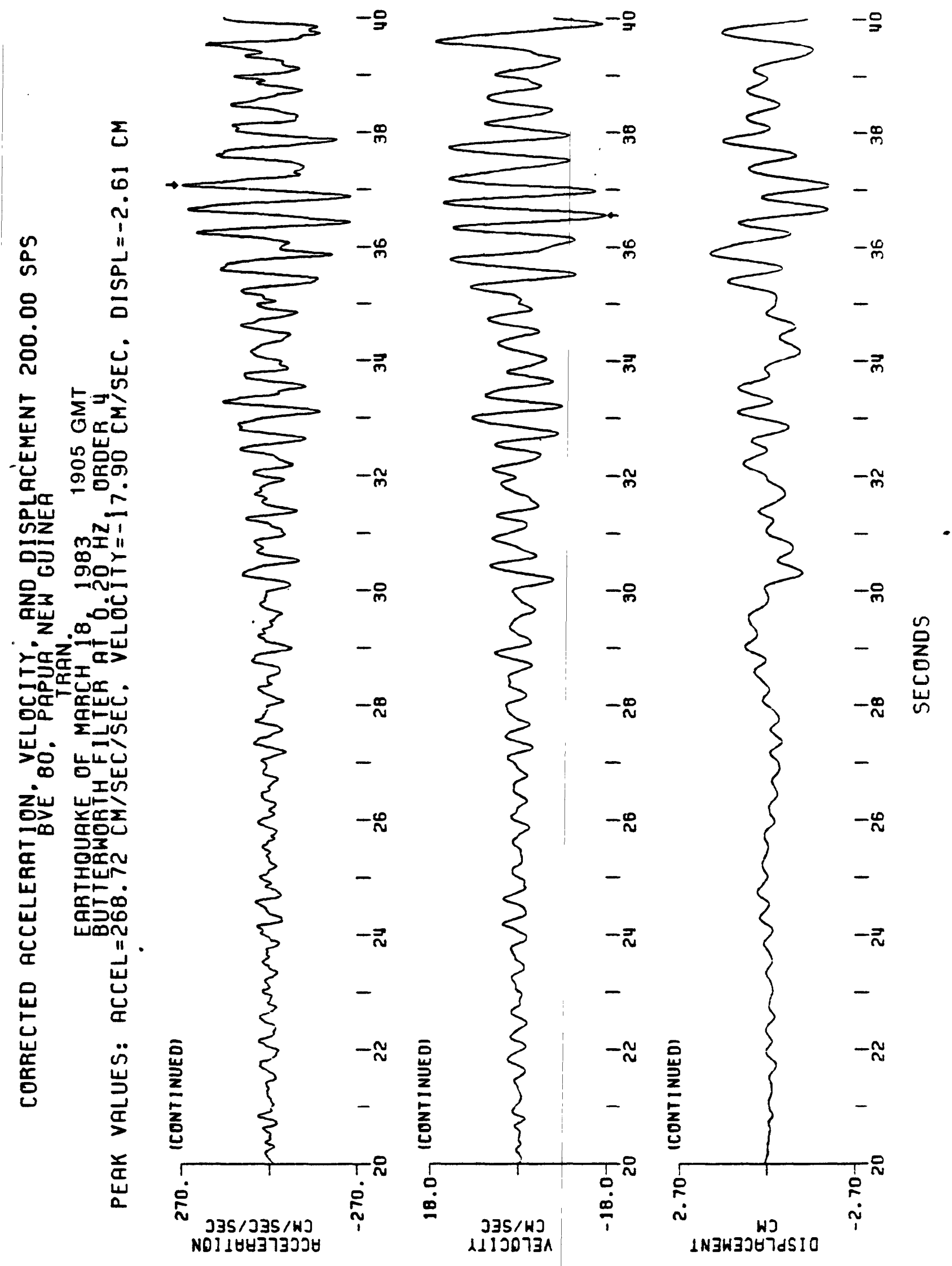




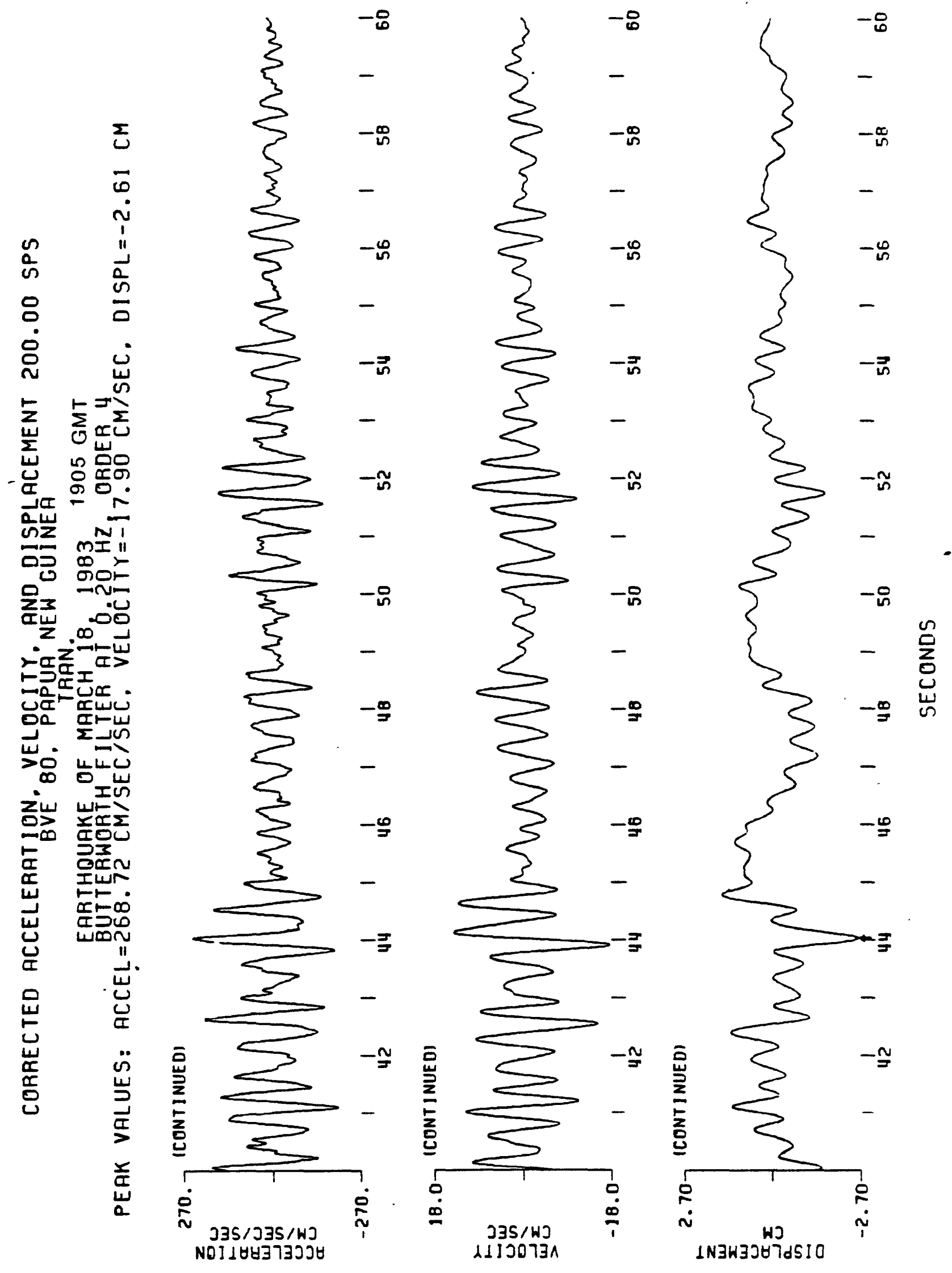




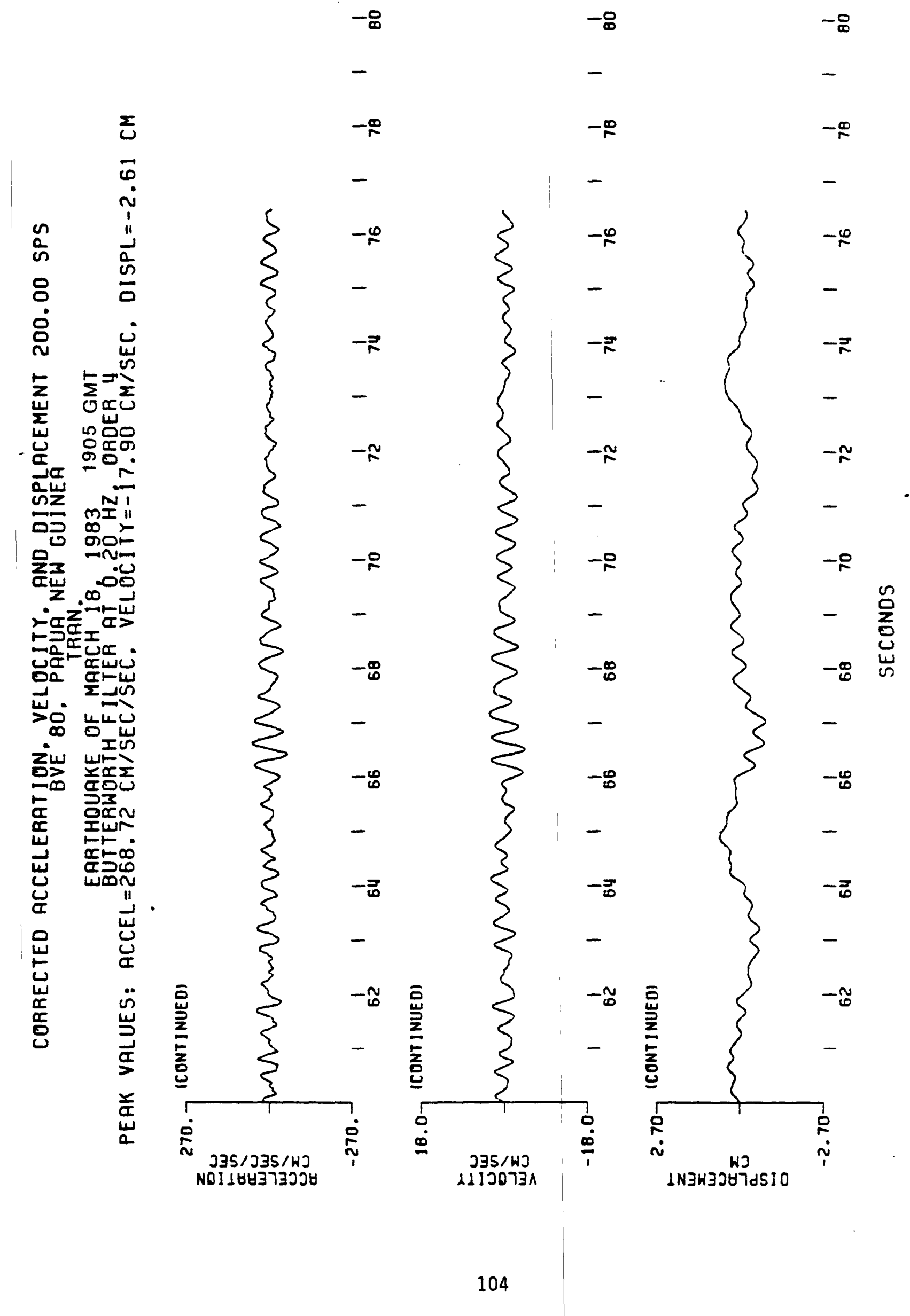




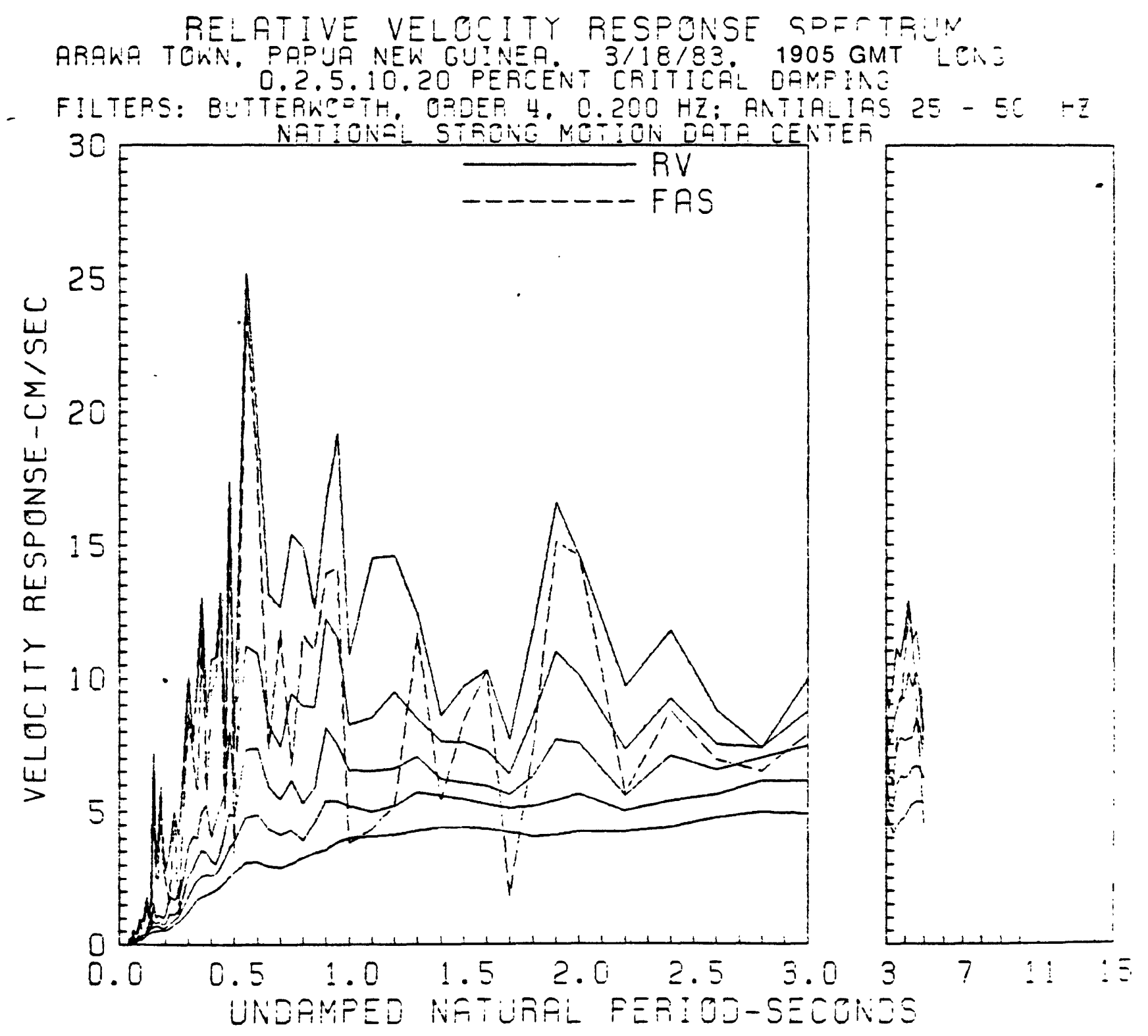




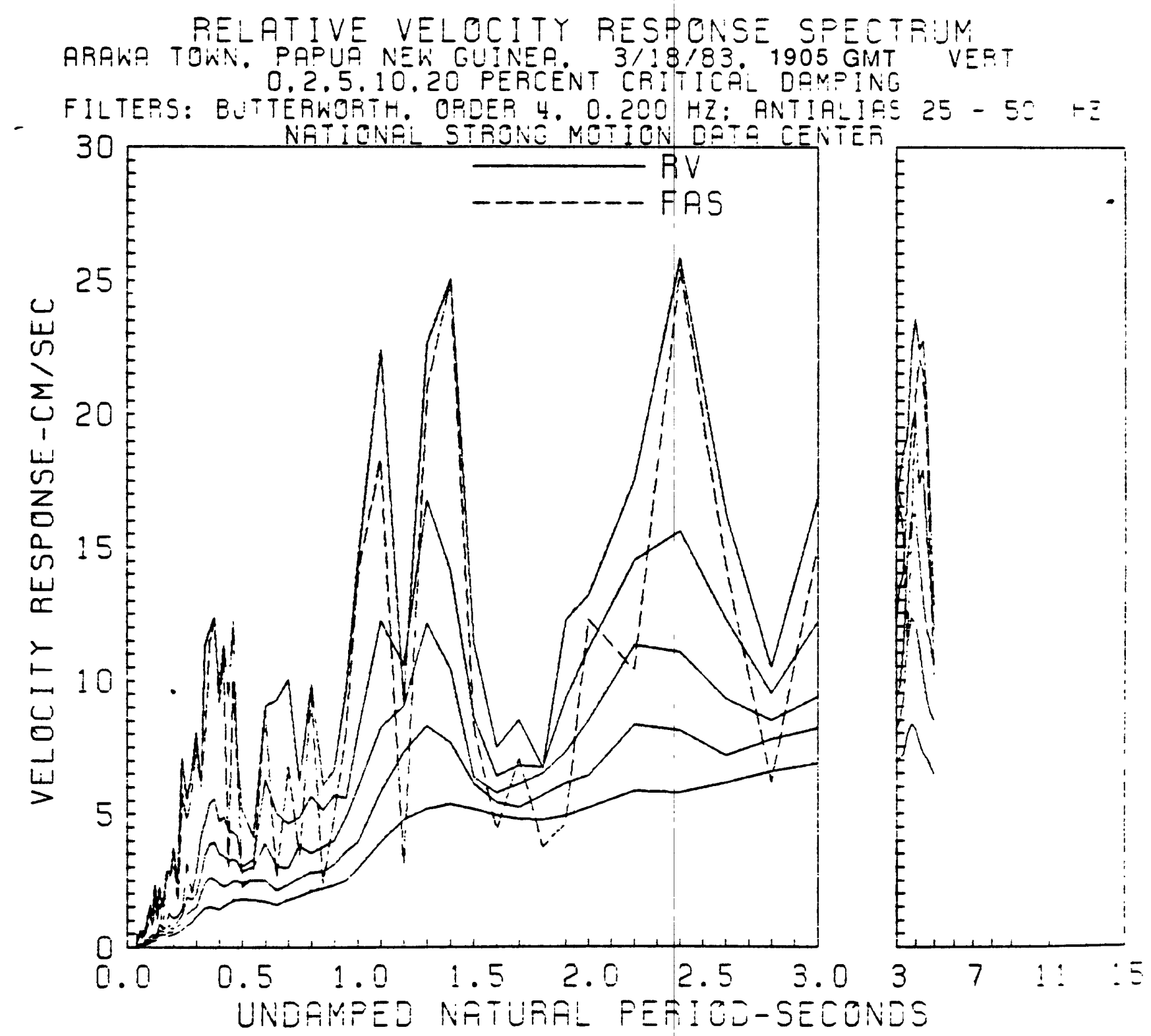




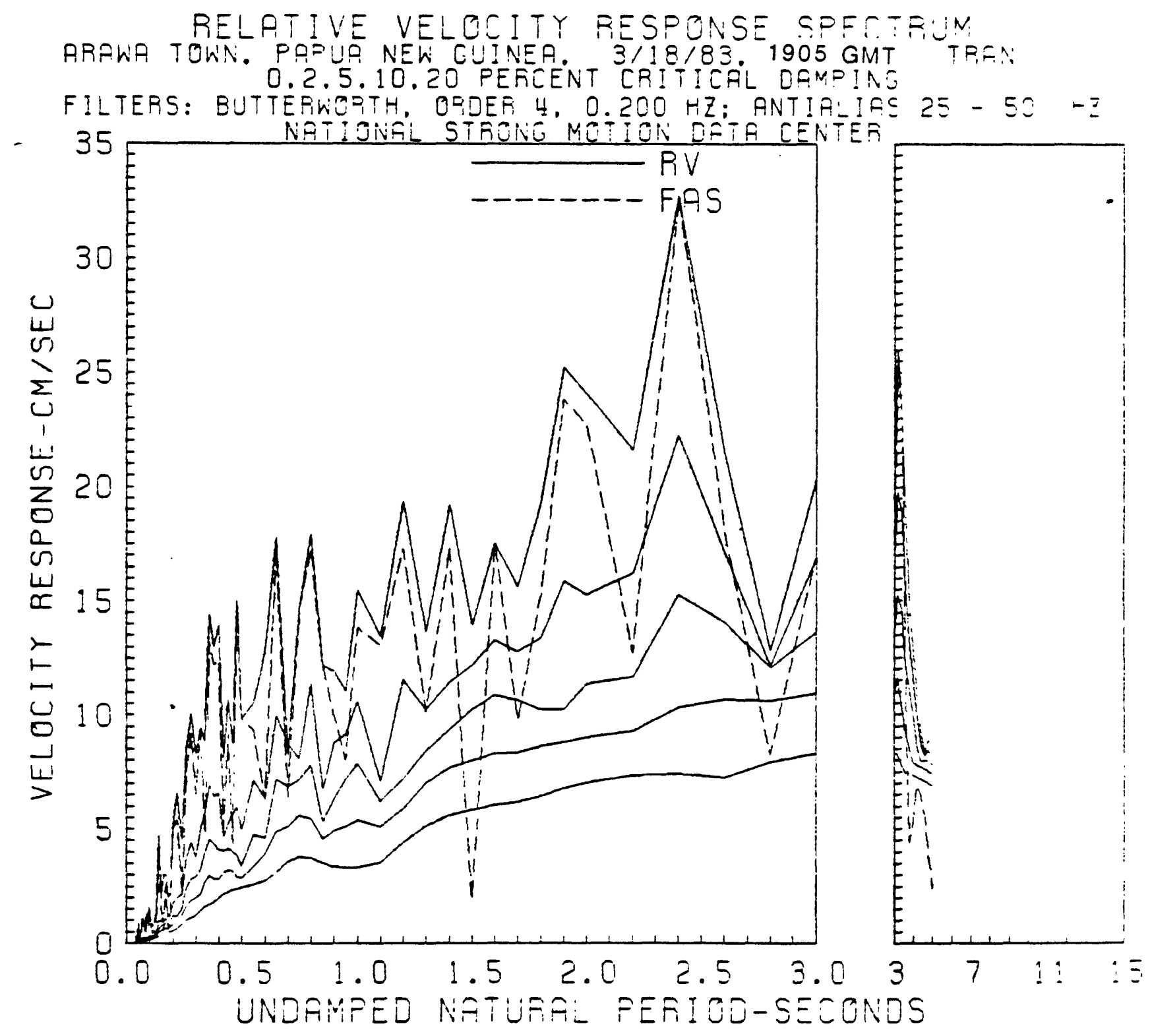


RELFTIVE VELOCITY RESPONSE SPECTPIN BATO BRIDCE. PAPUA NEW GUINEA 3/18/83. 1905 GMT LCNE

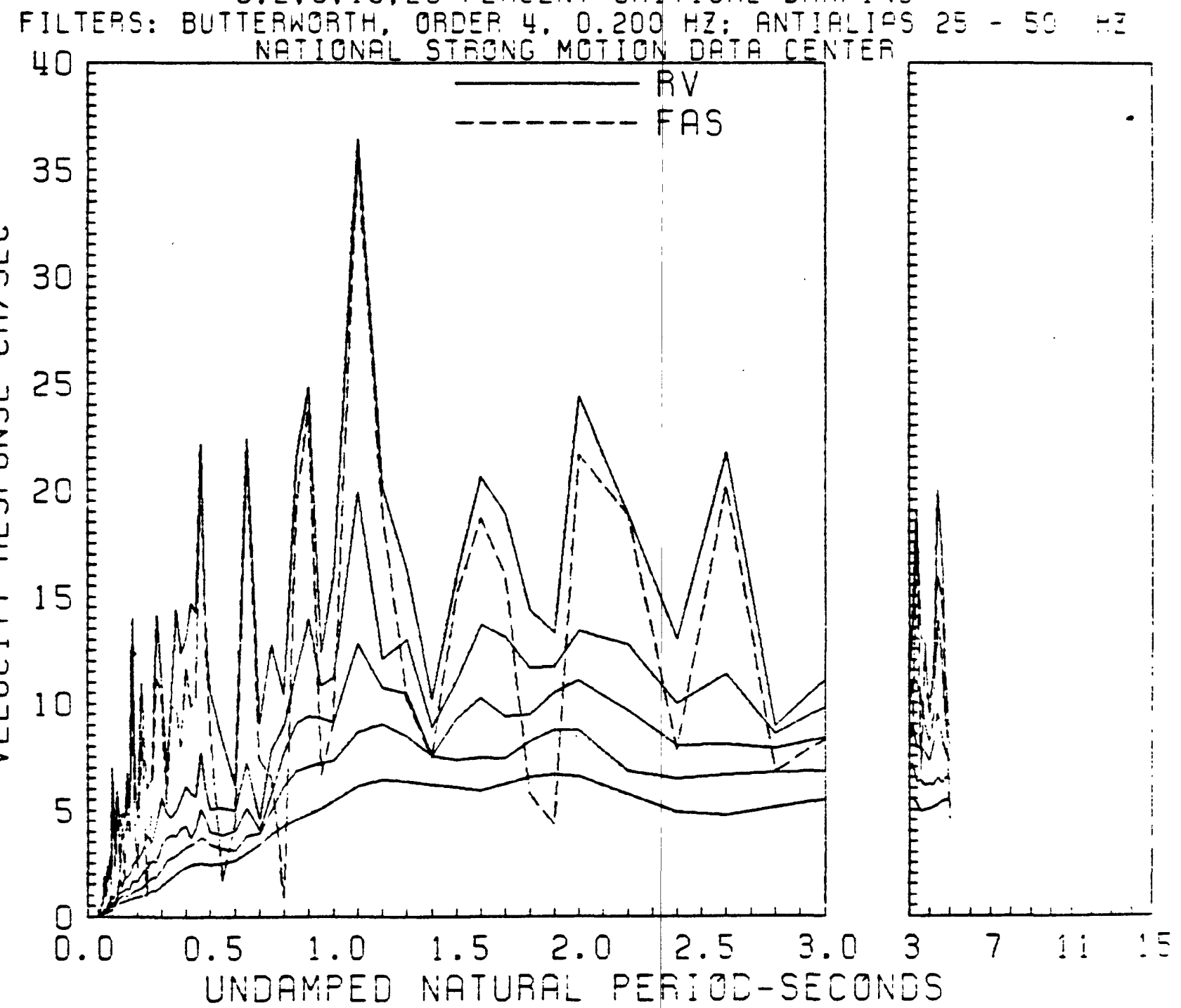




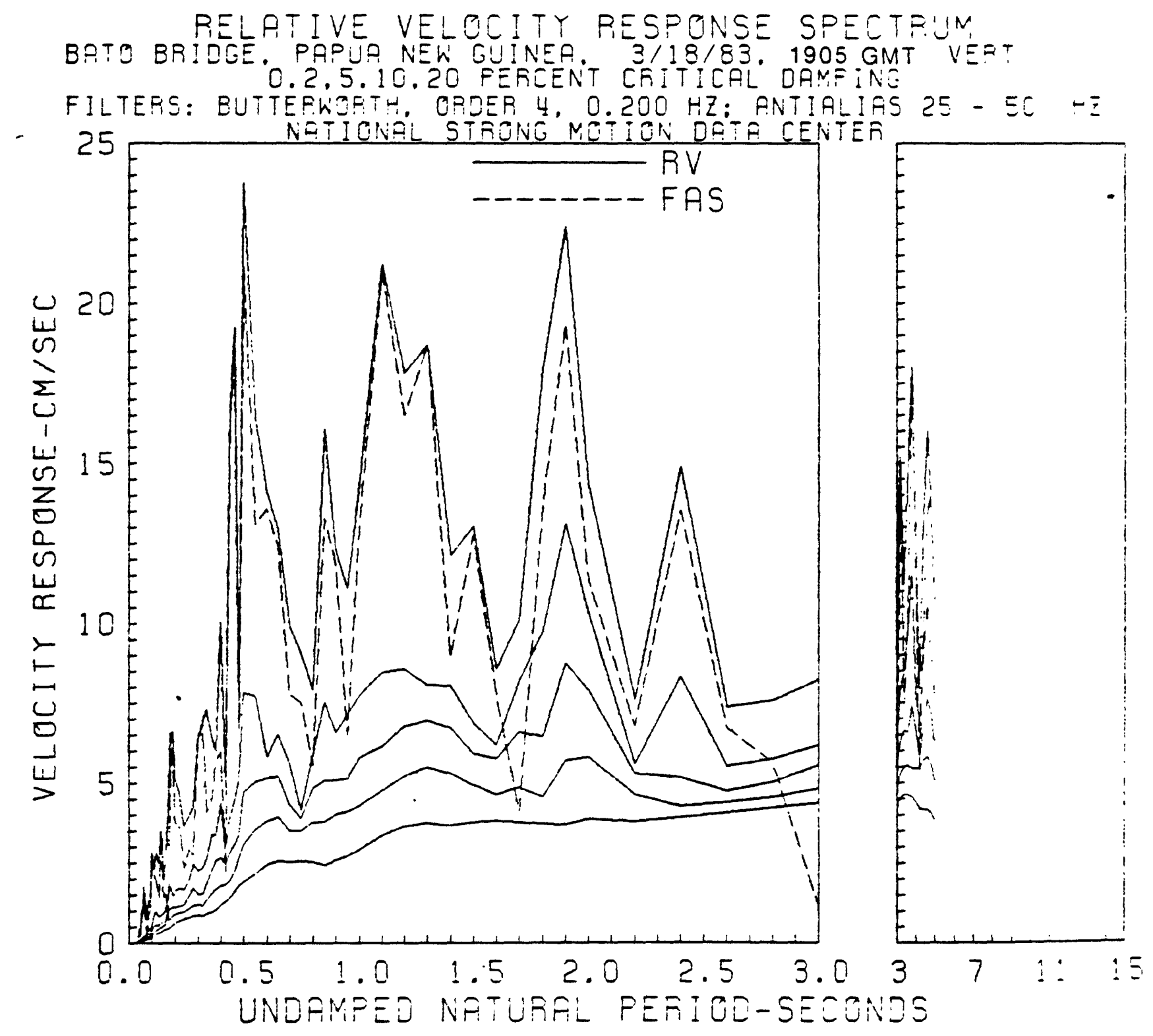


RELATIVE VELOCITY RESPONSE SPECTRUN BATO BRIDGE. PFPUA NEW CUINEA 3,18/83. 1905 GMT TRFA $0.2 .5,10.20$ PEREENT CAITICA DFMFING

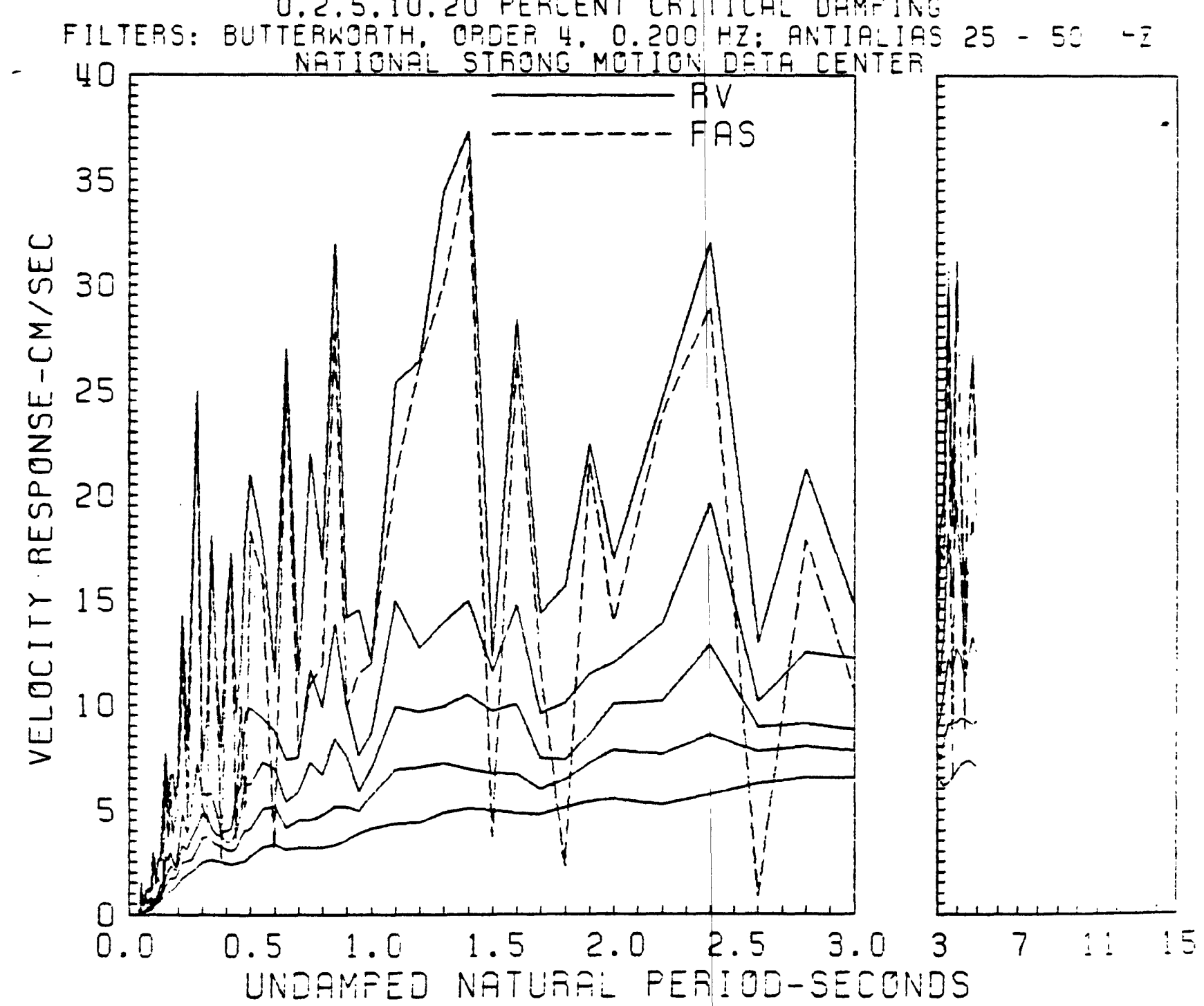


RELATIVE VELOCITY RESPONSE SPFCTFUN

BVE 8O. PAPUA NEW GLINEF, 3/18183. 1905 GMT LCNO

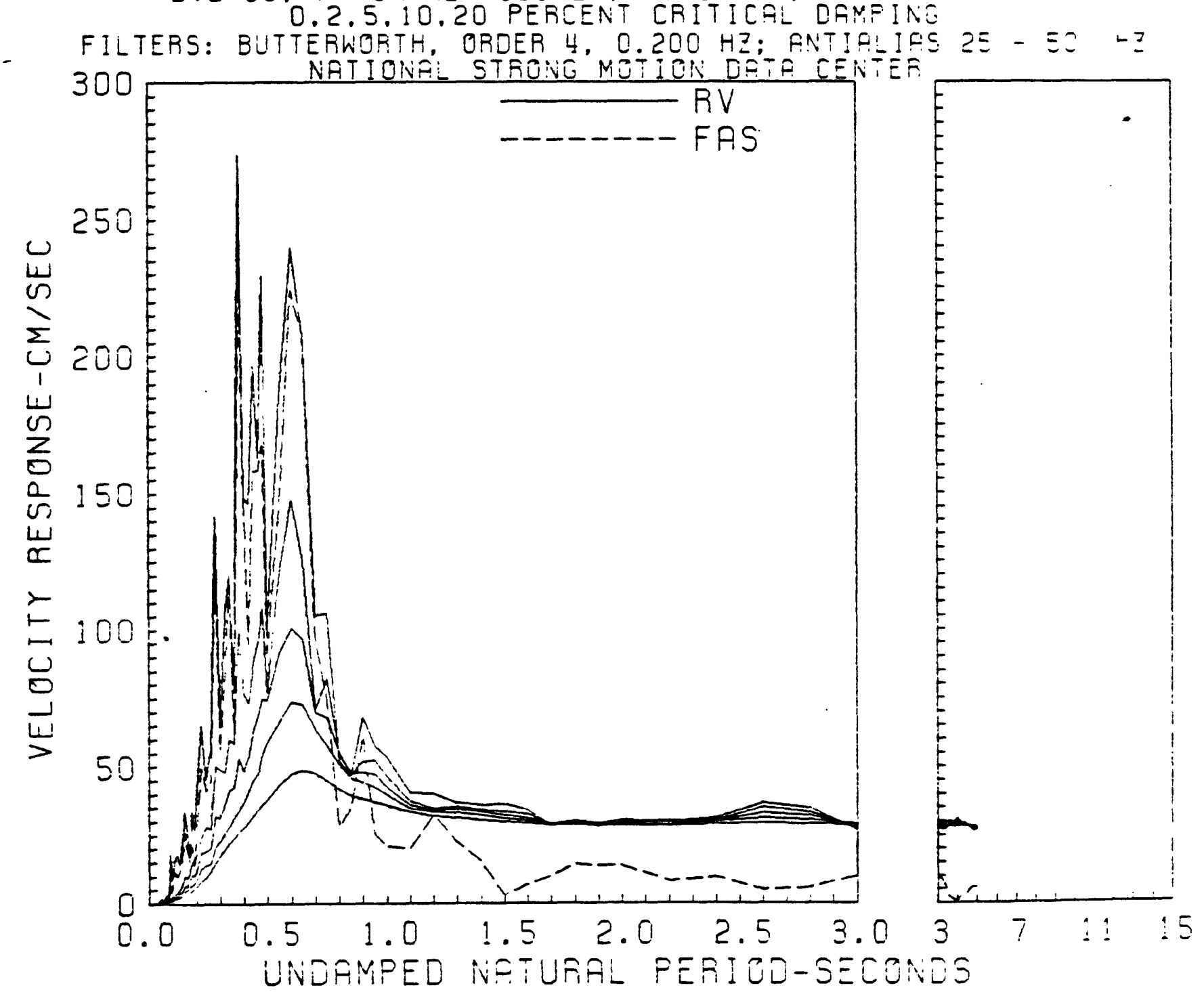




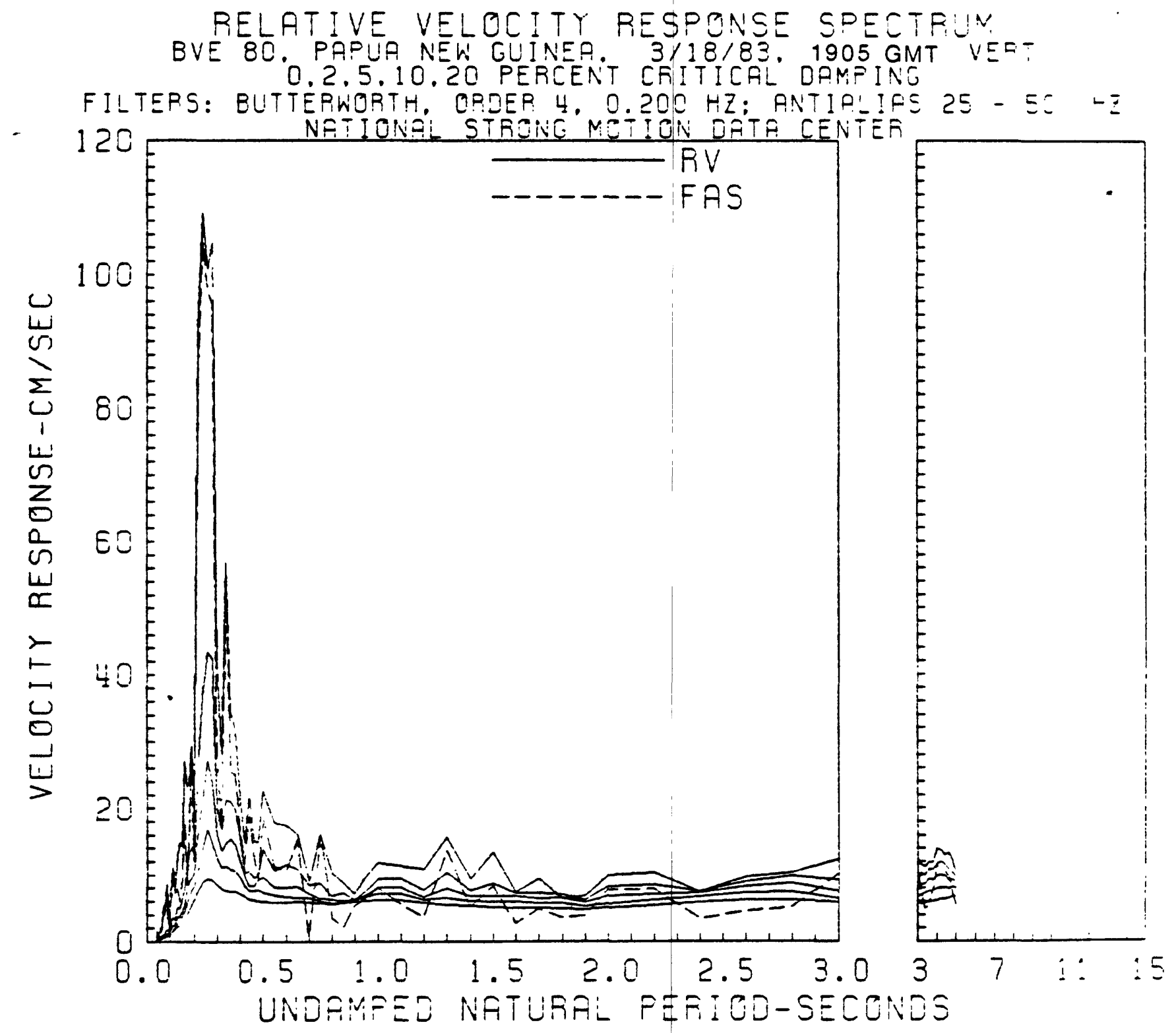




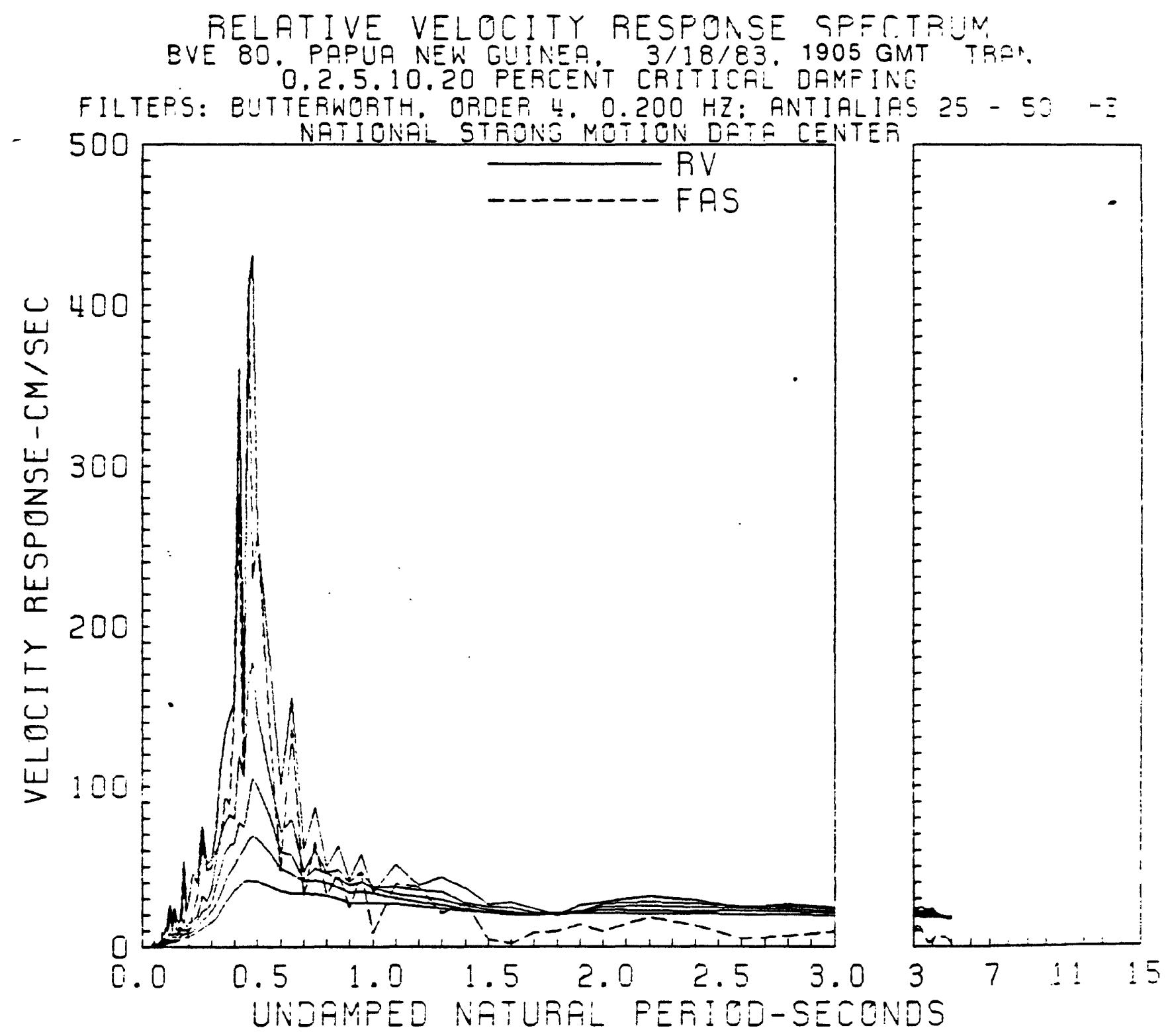




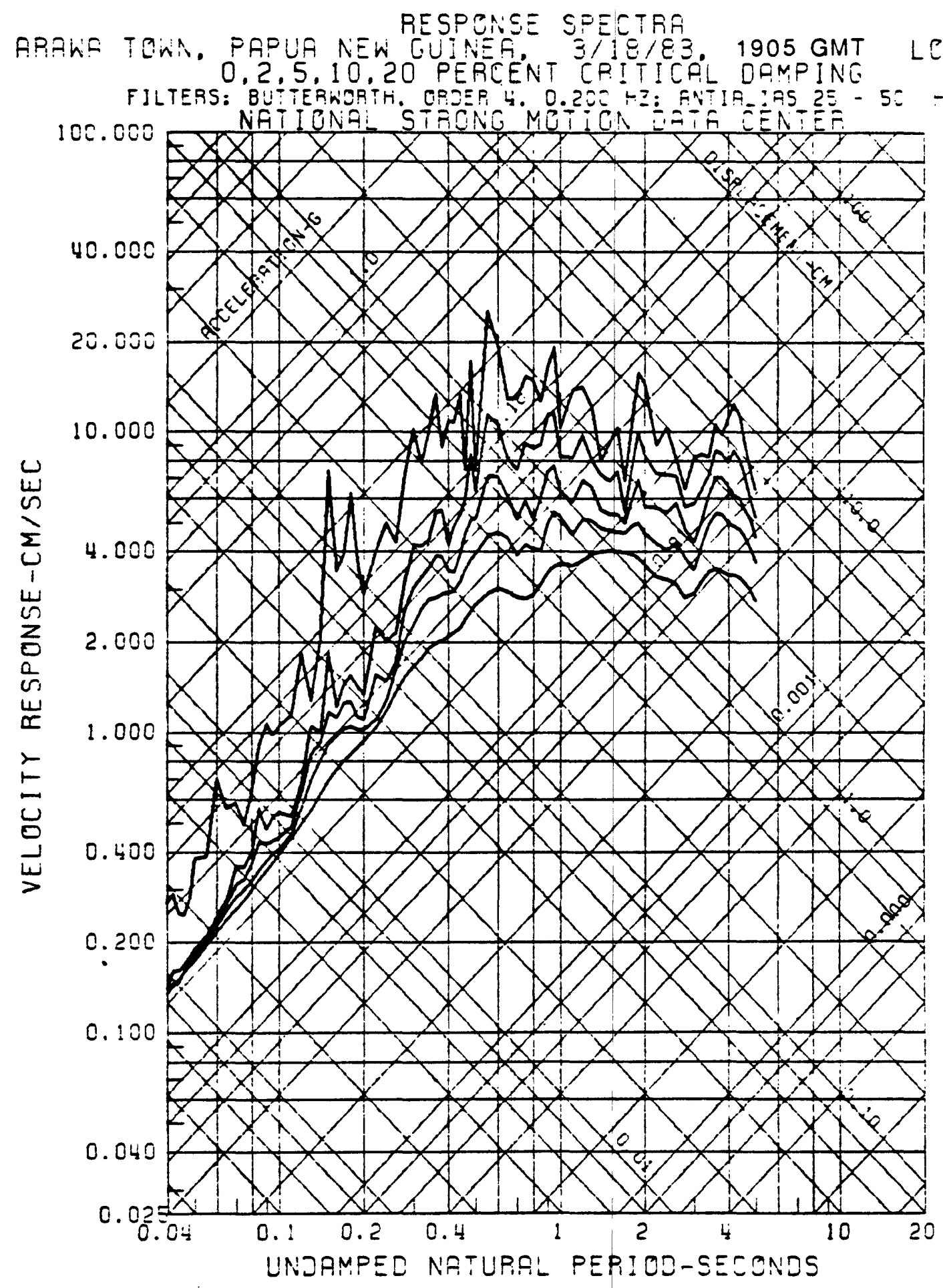


PIESPCNSE SFECTRF

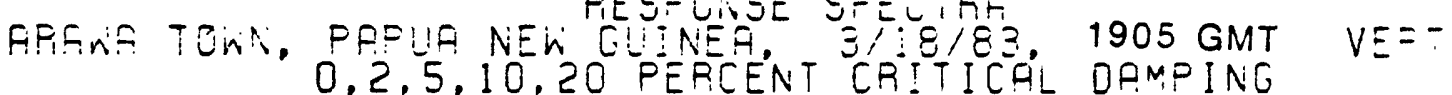

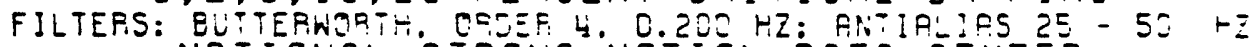

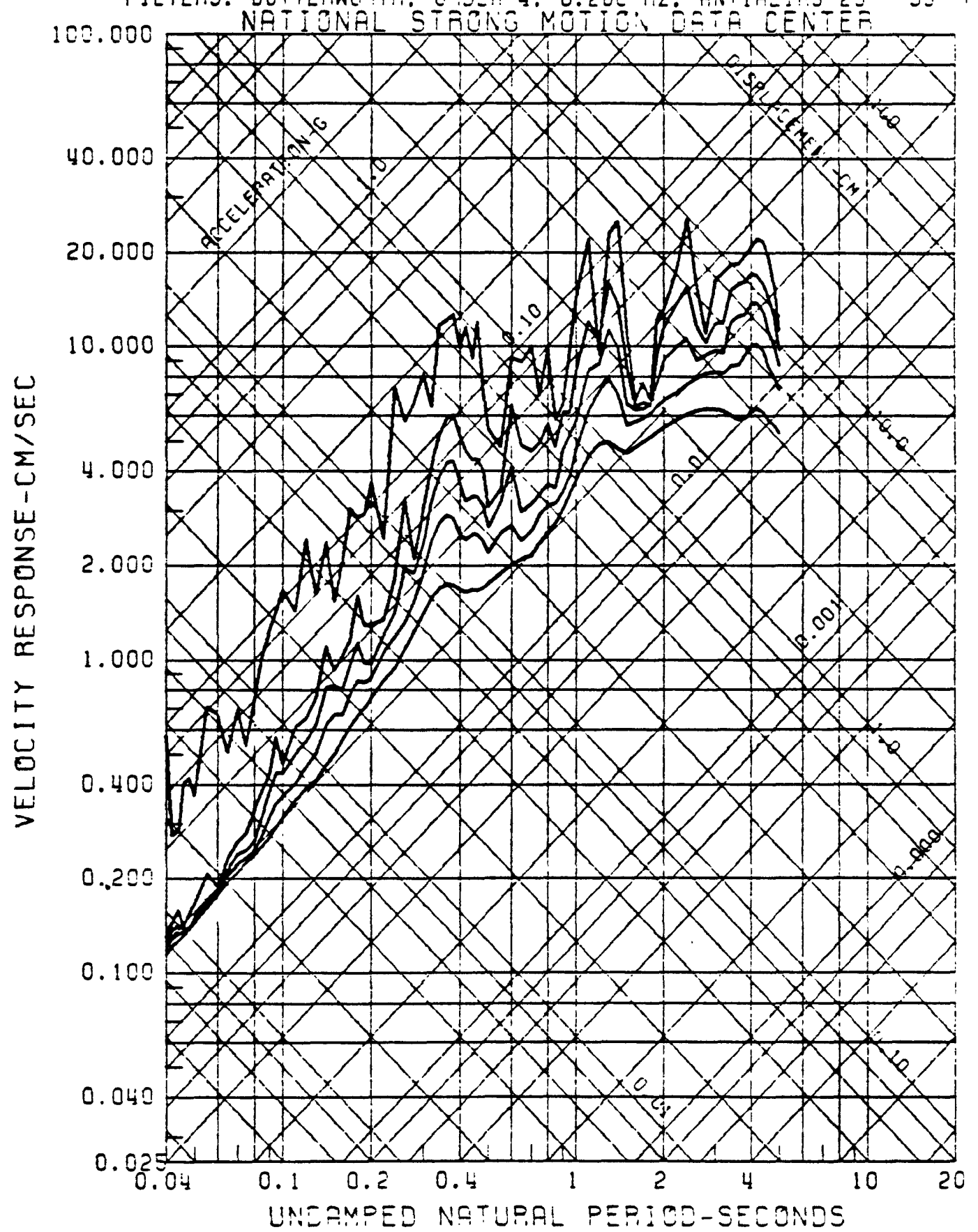




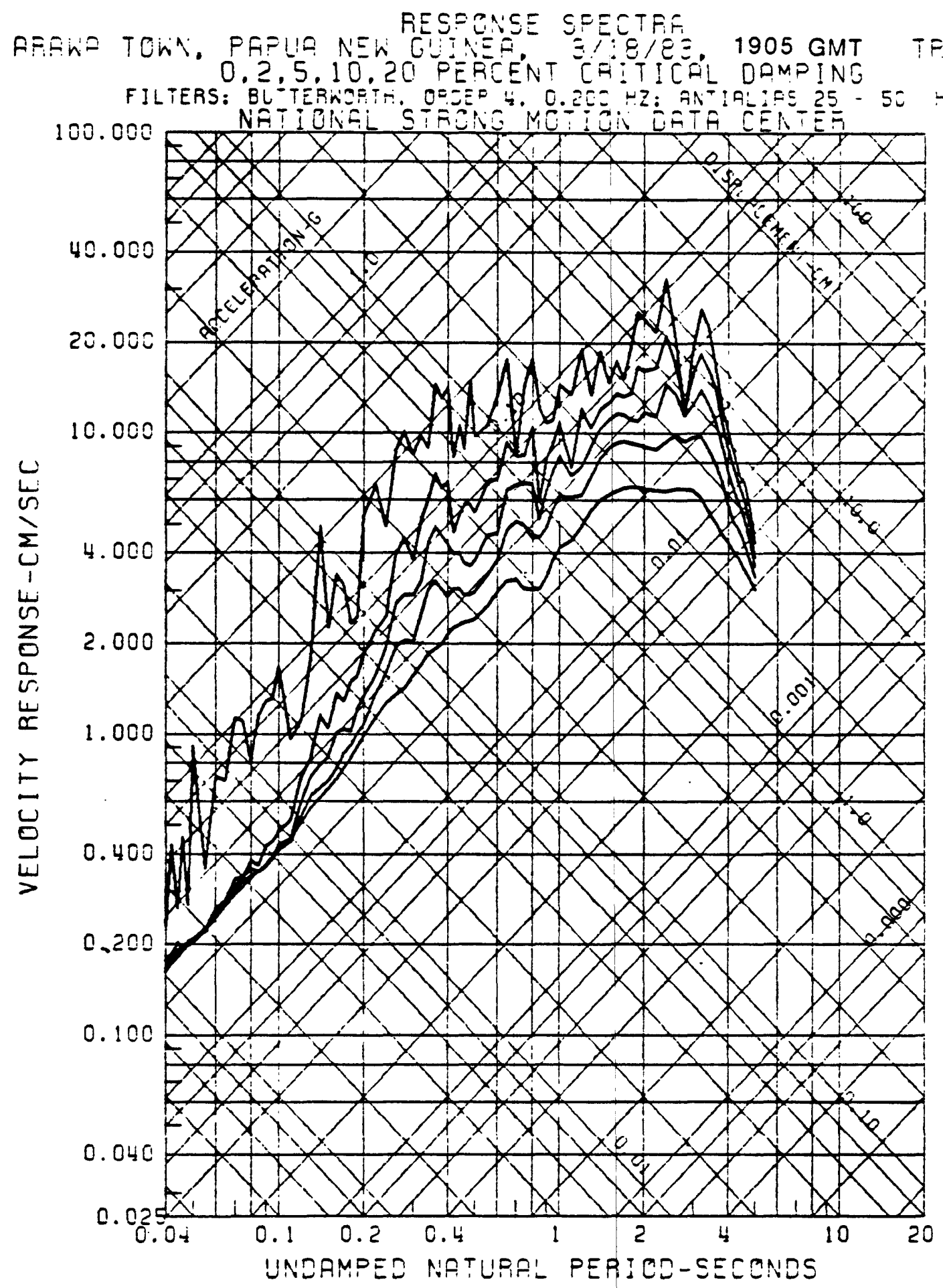




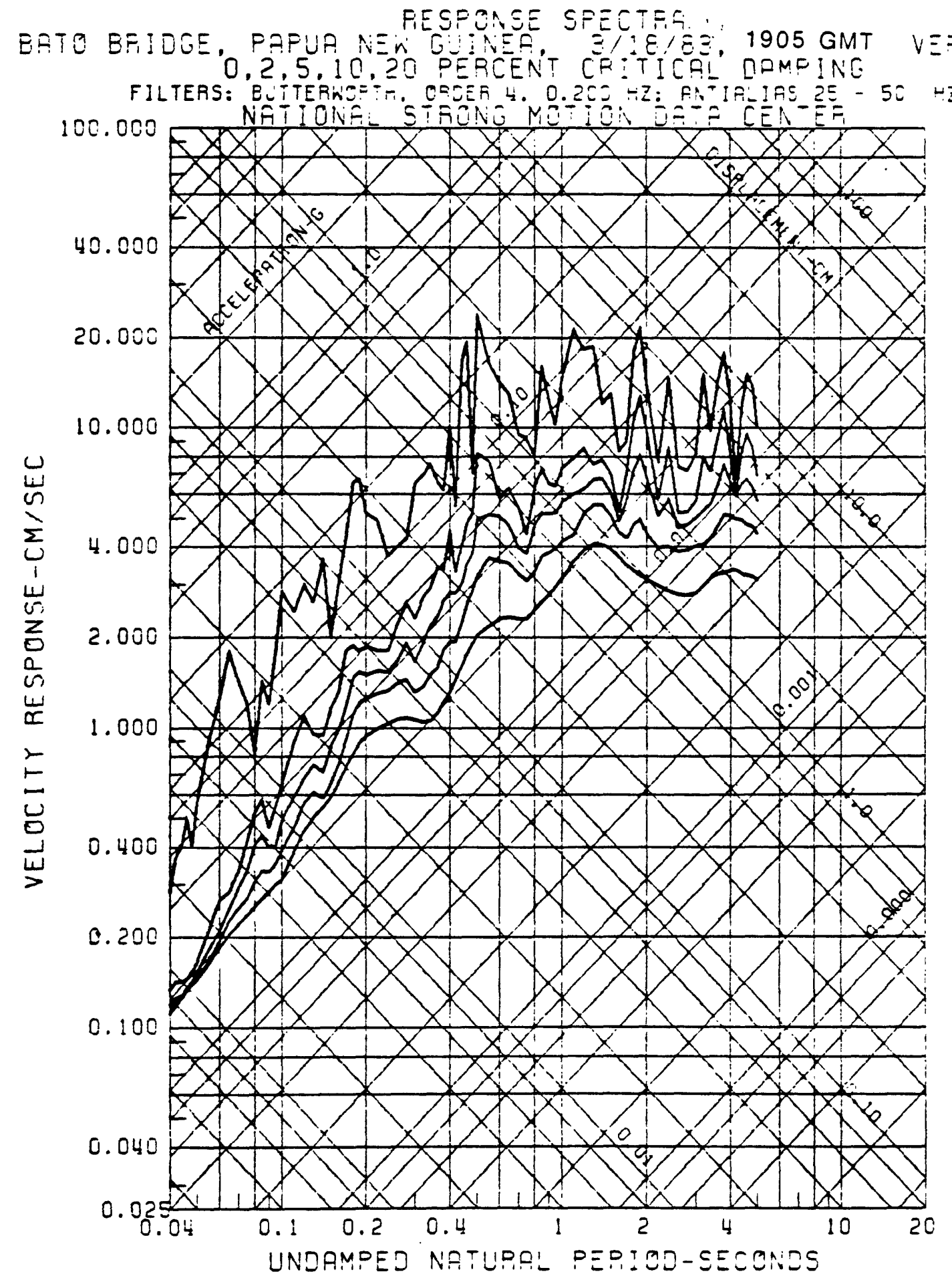


BATC EEIDSE, PAPUP NEA GUINEA SFECTFA

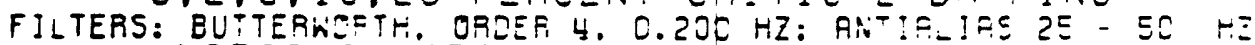

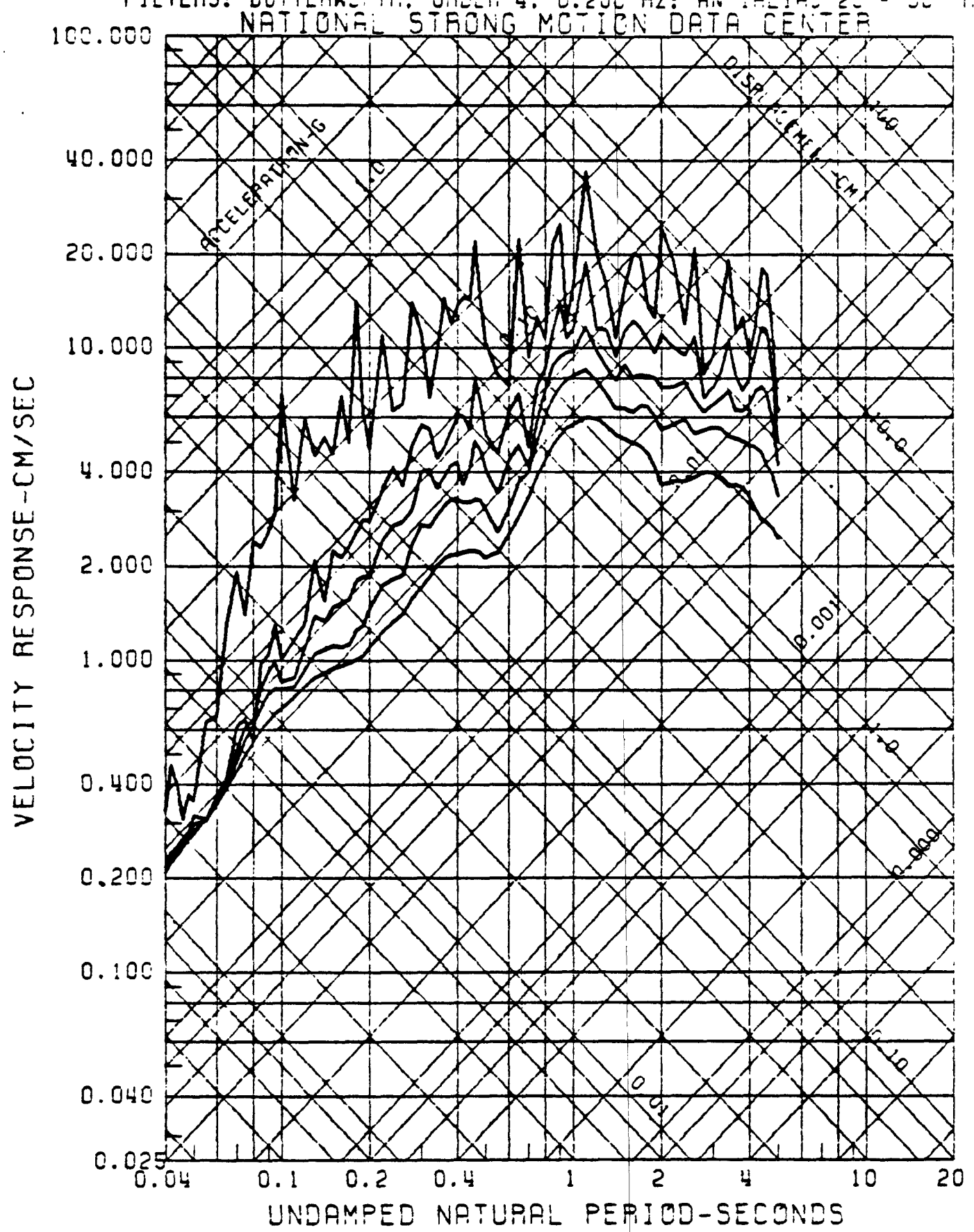




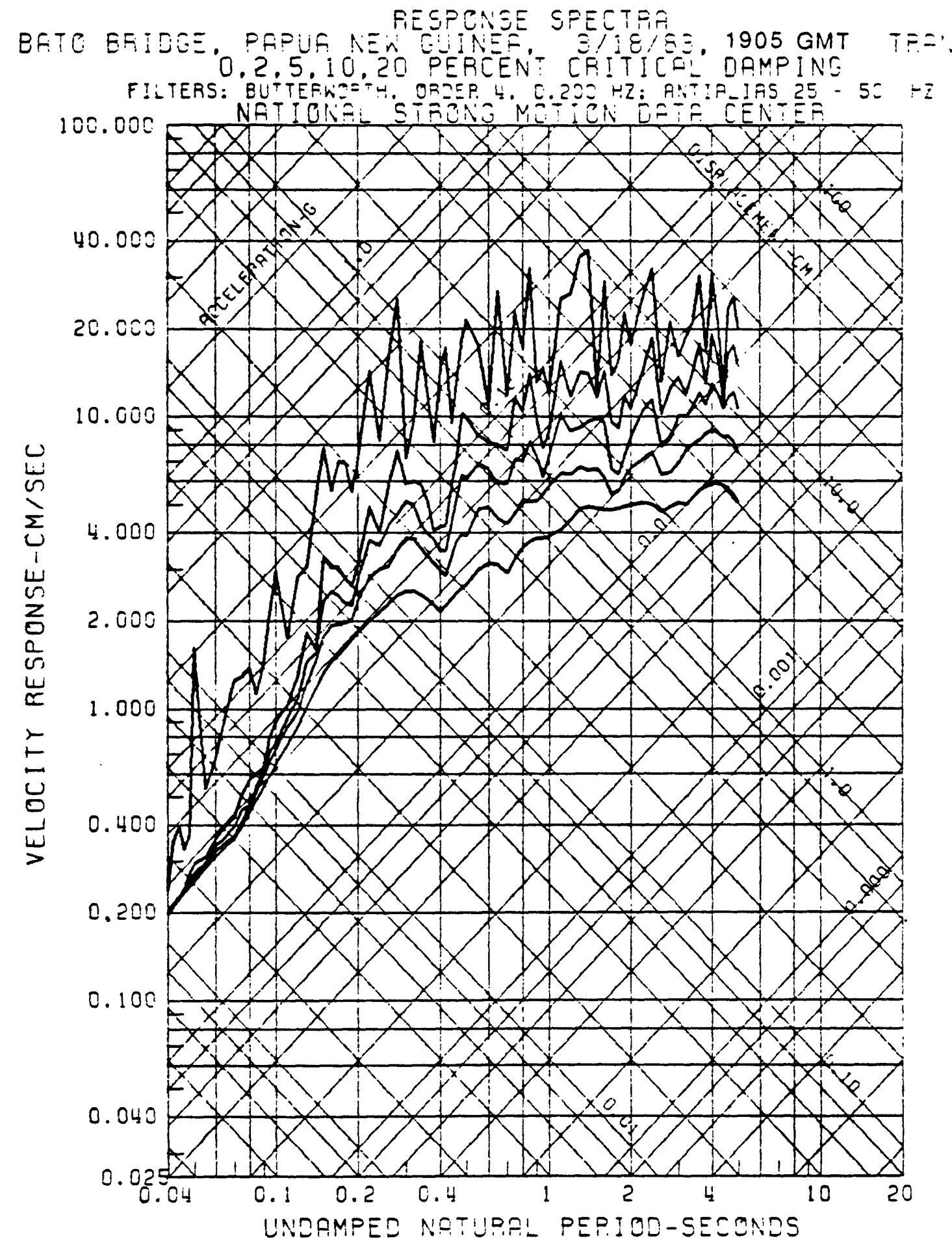




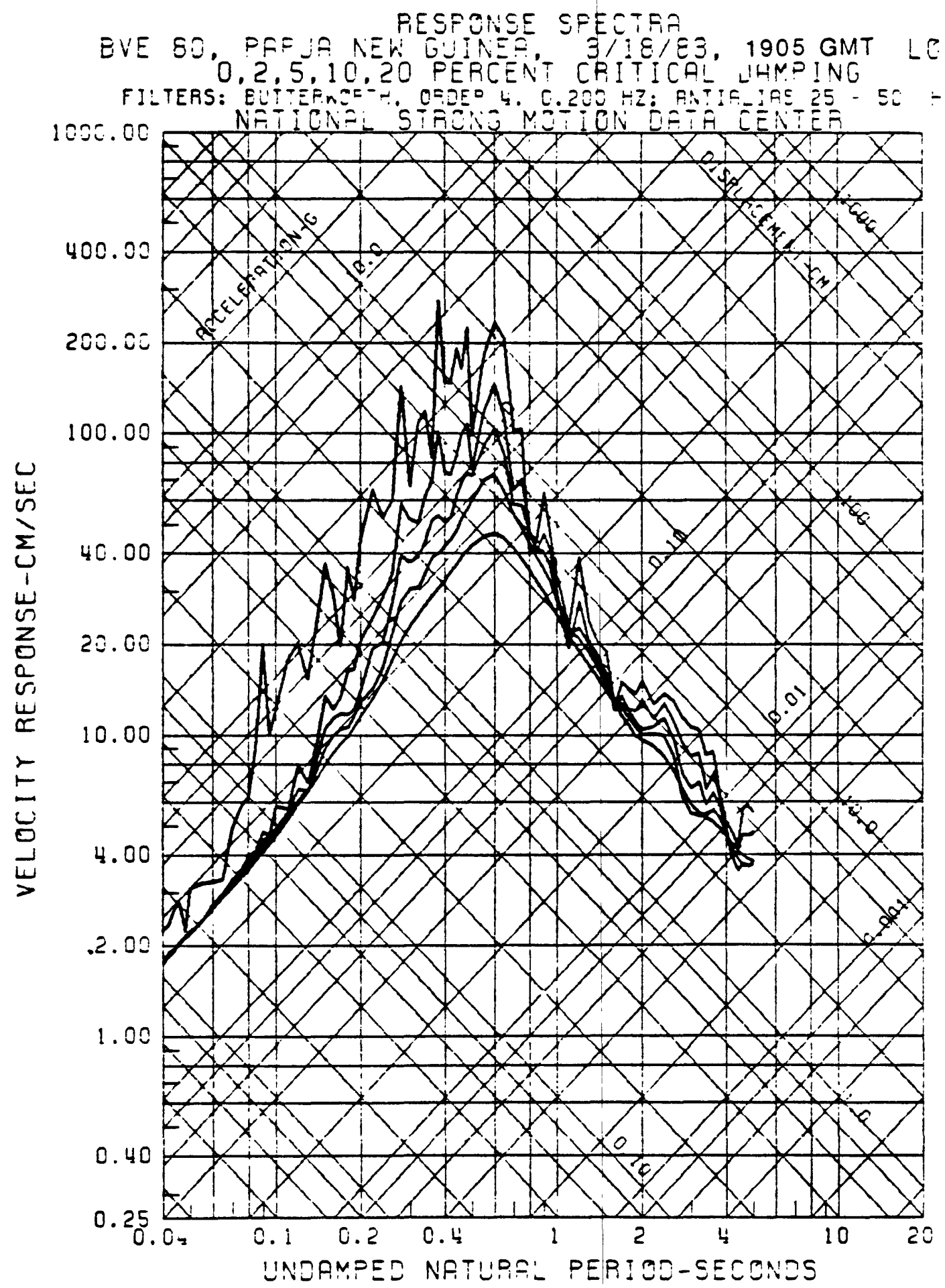




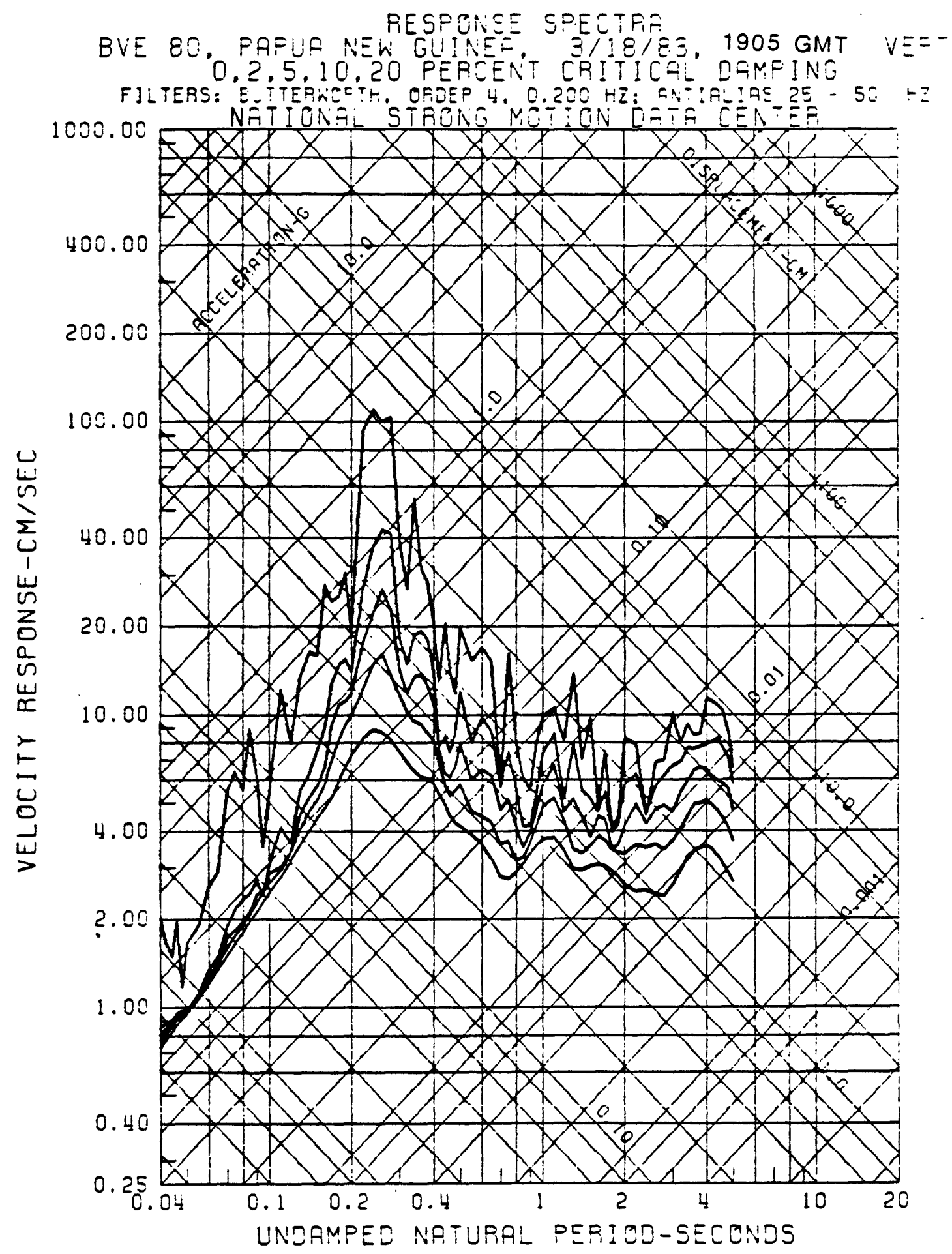




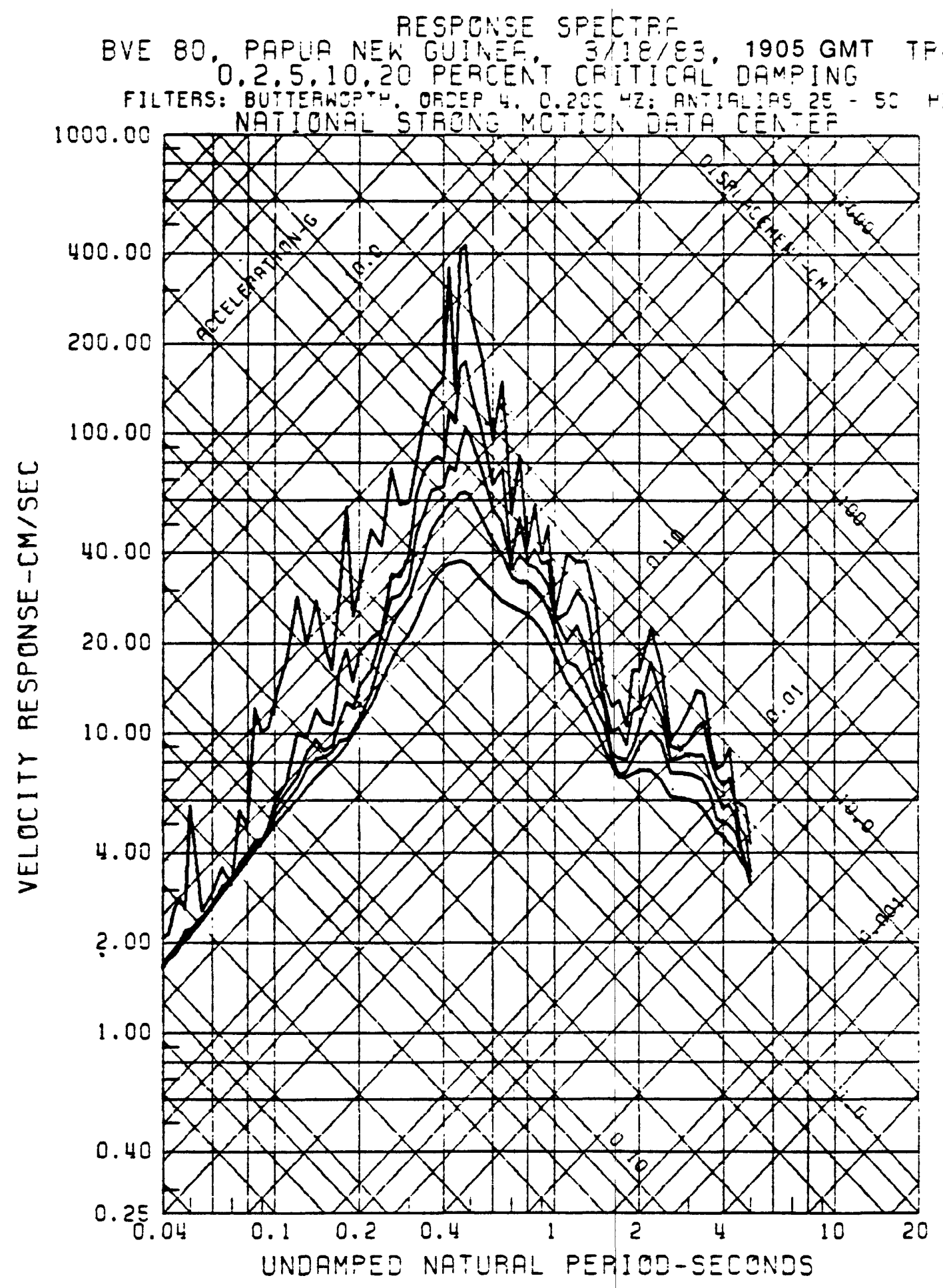




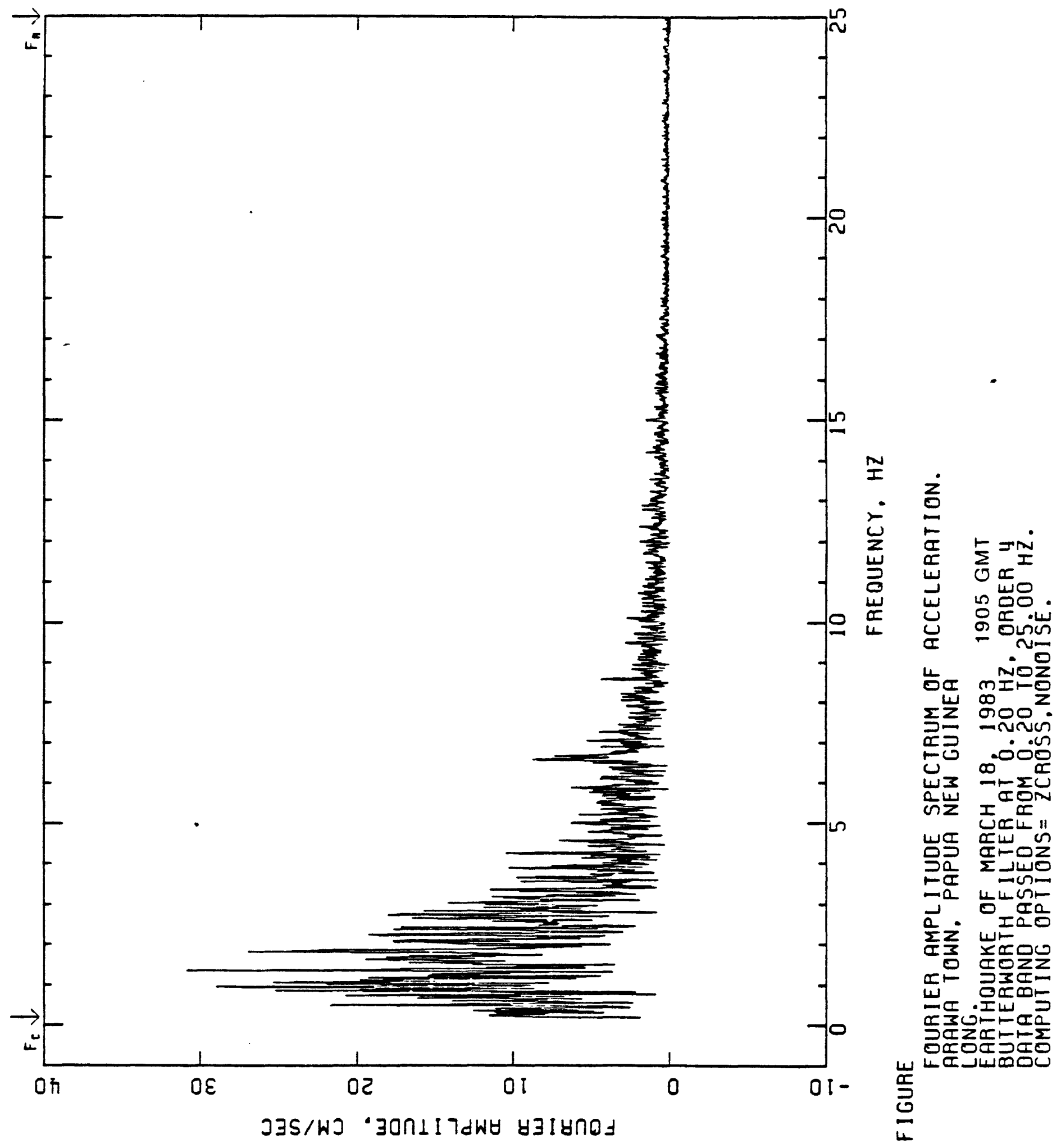




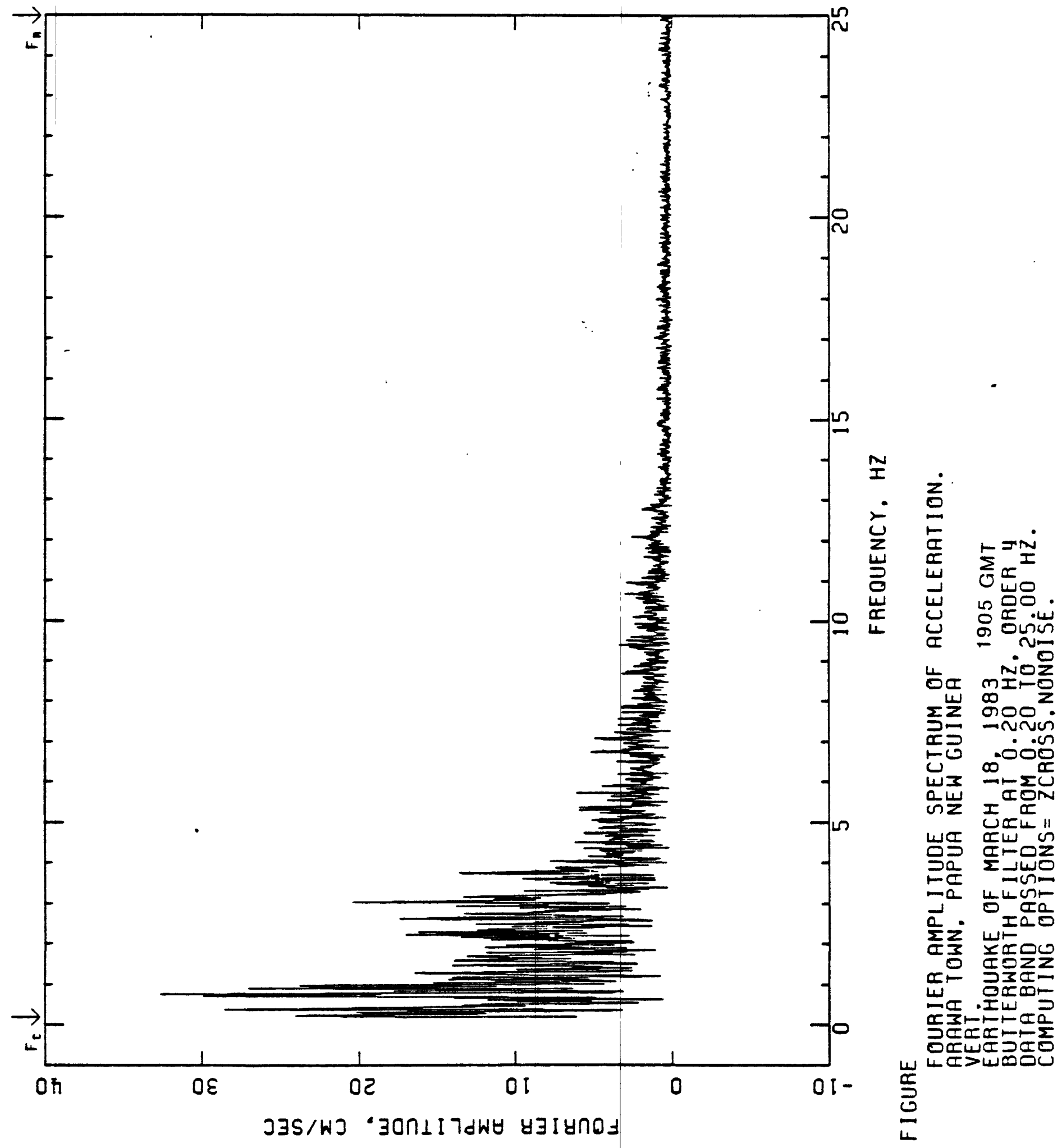




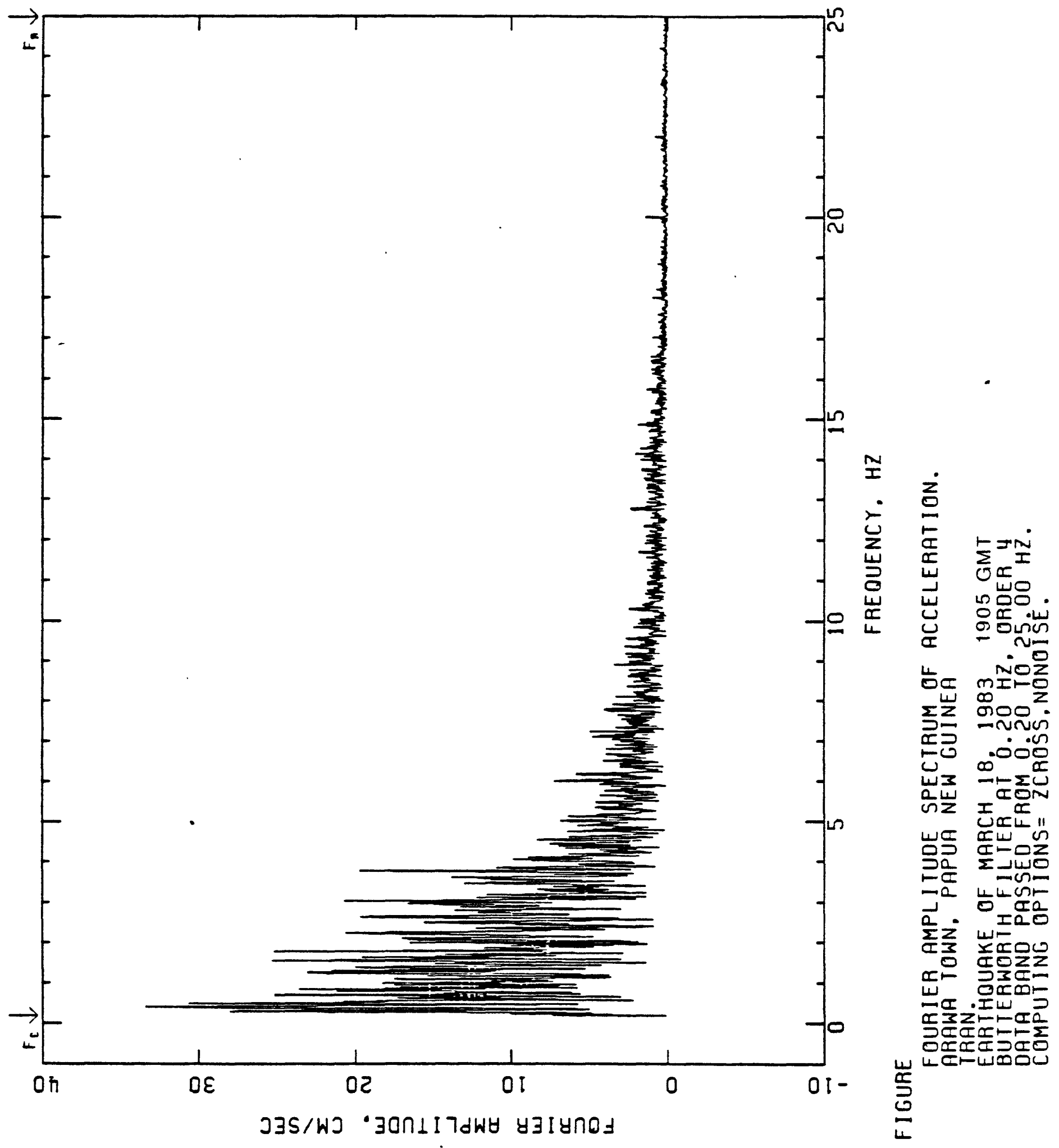




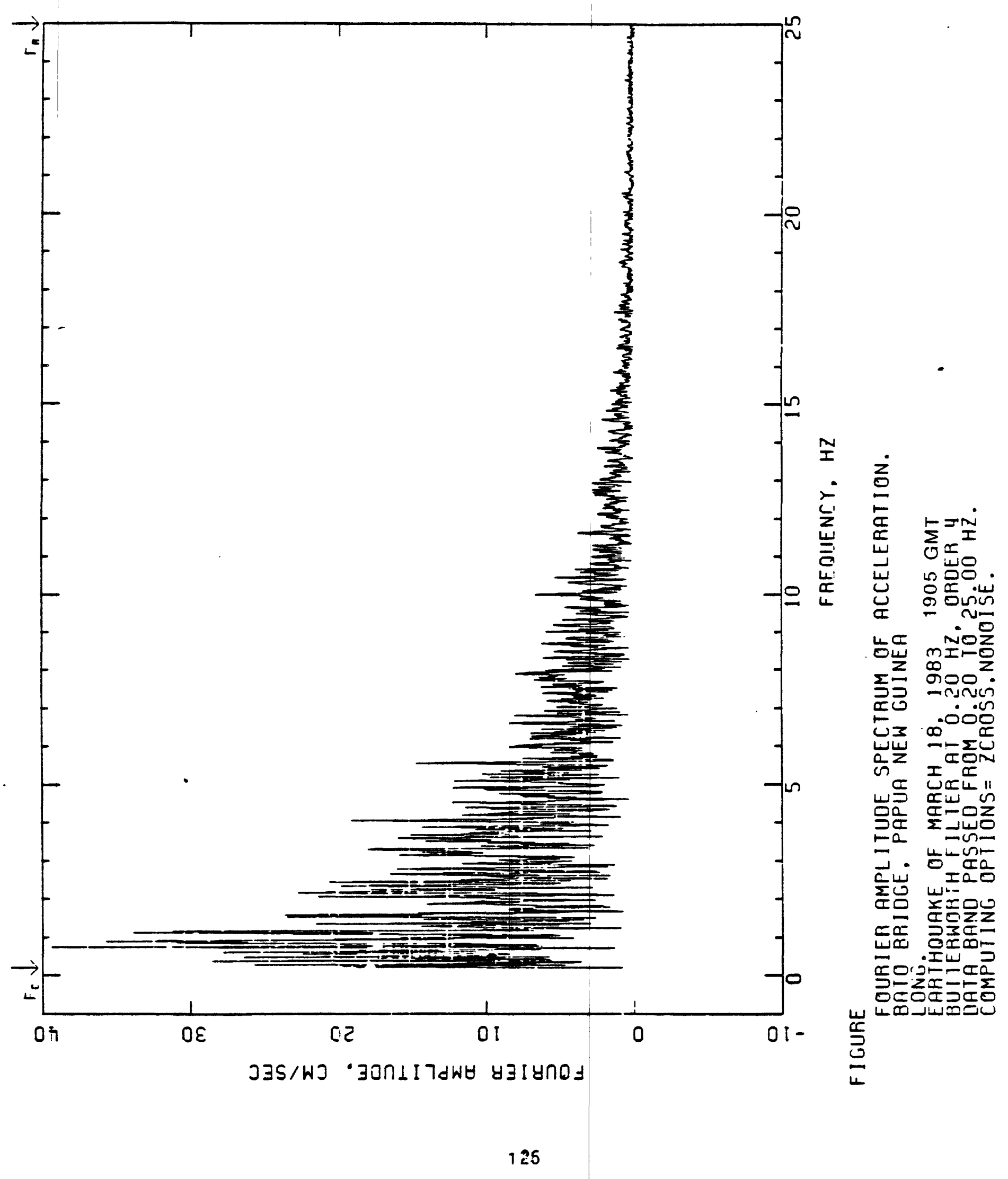




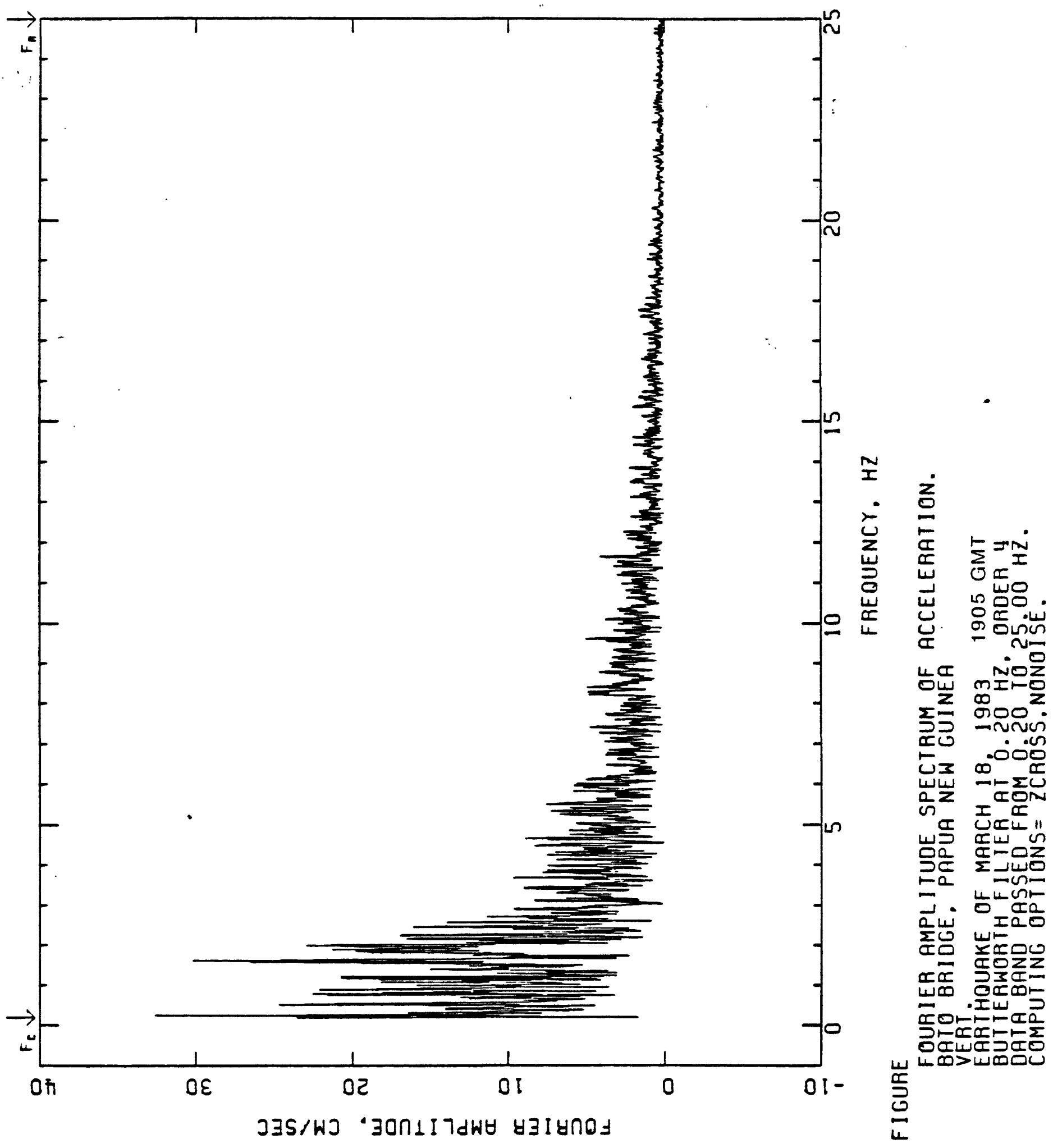




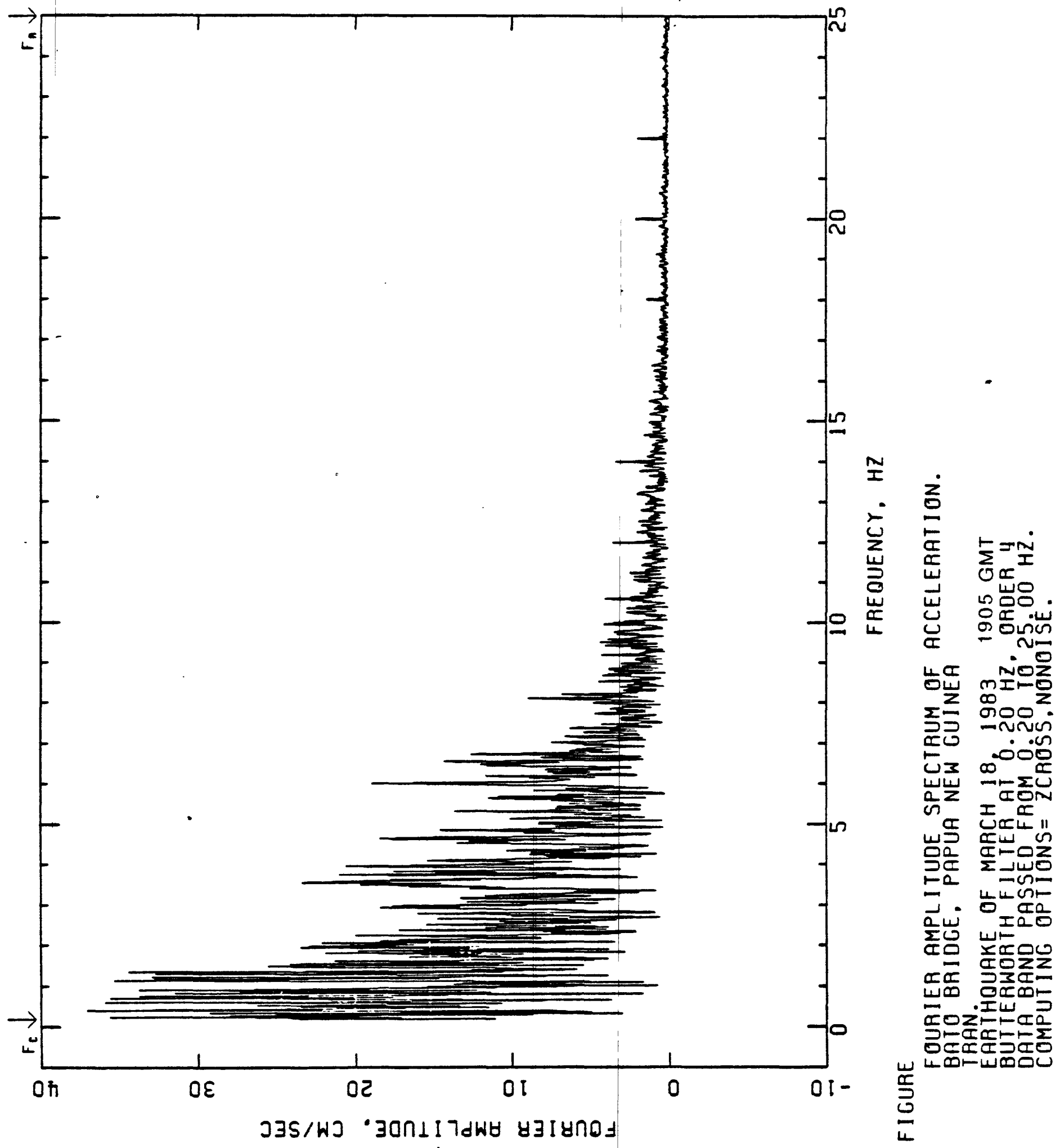




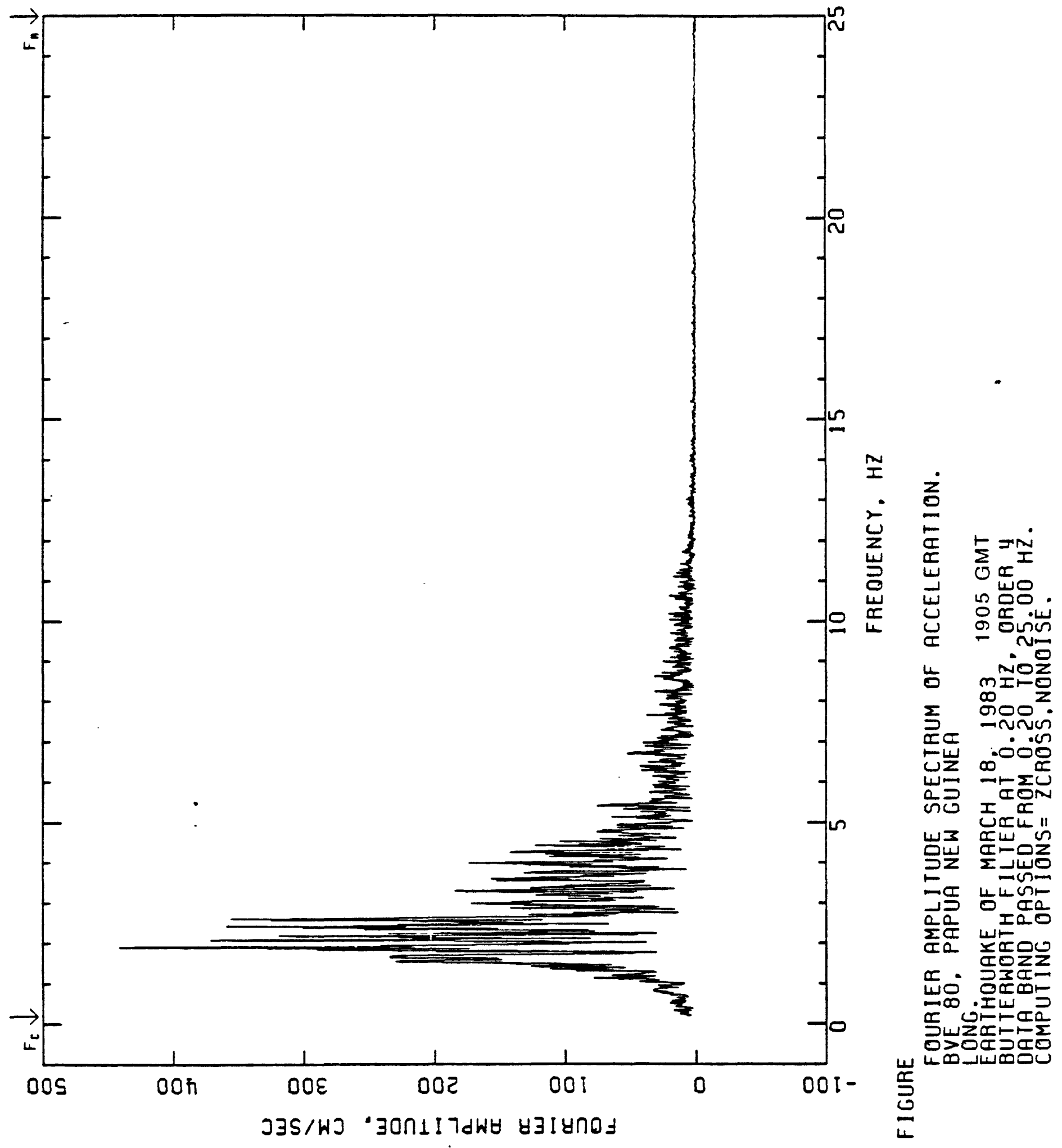




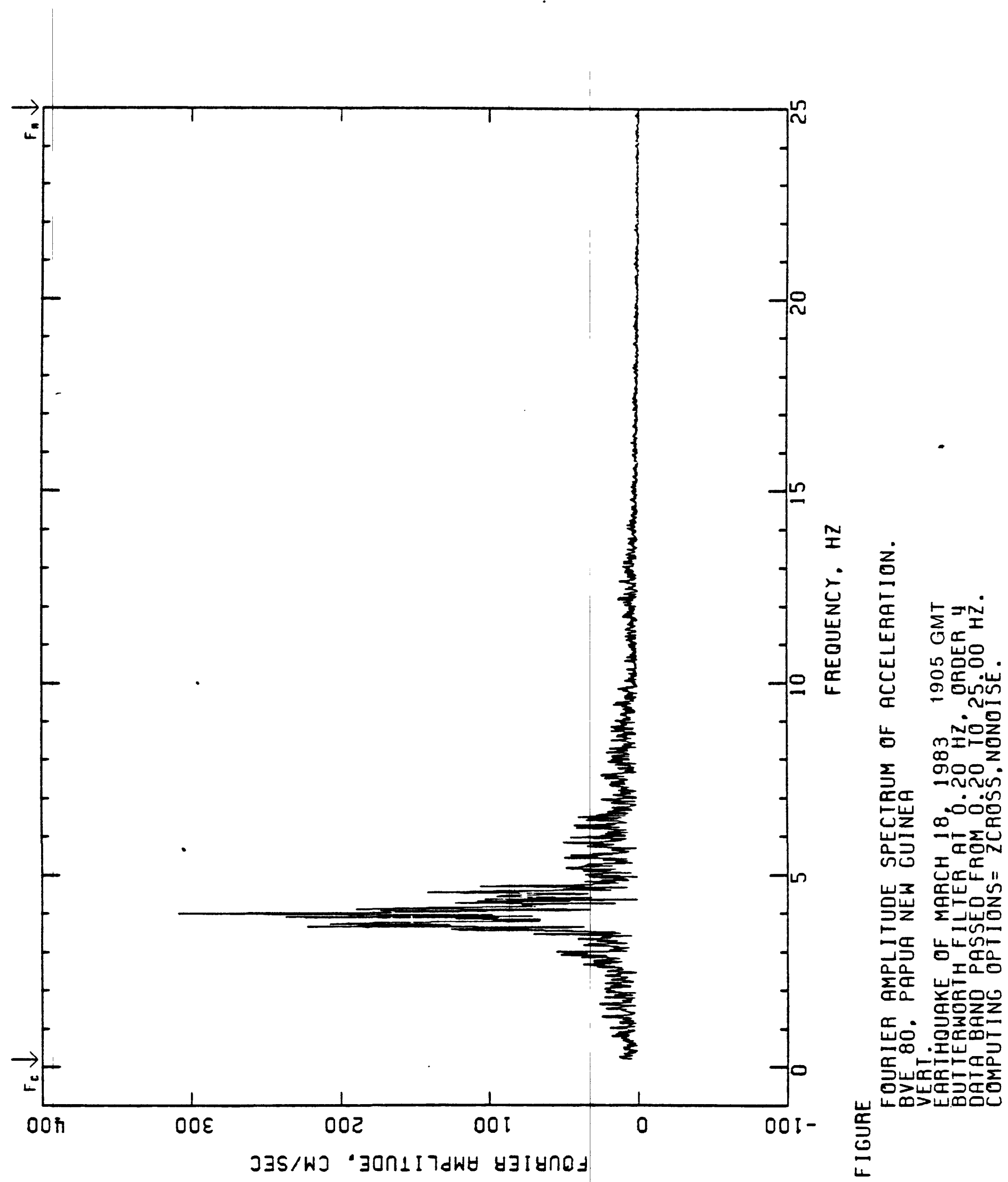




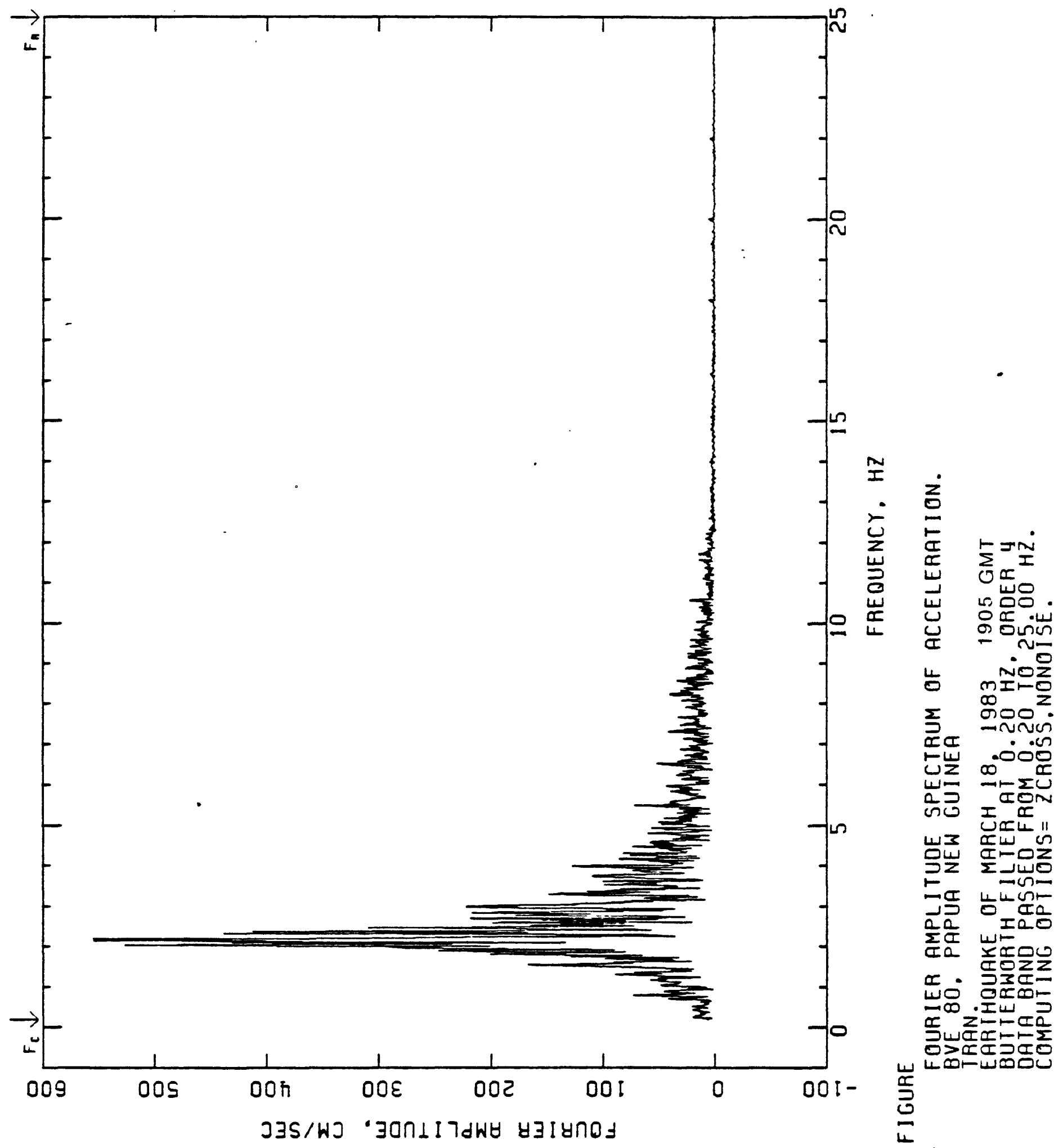




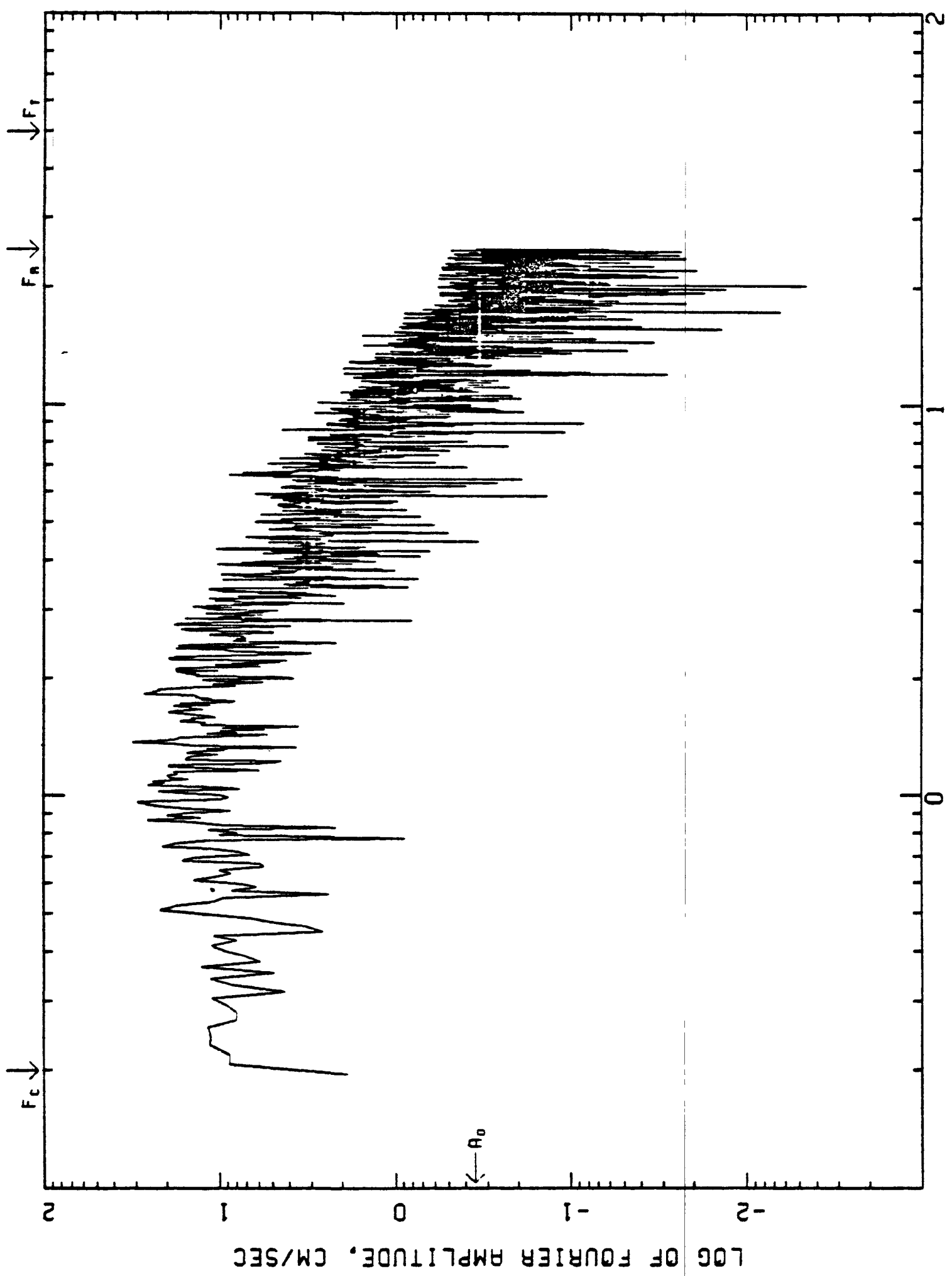

采

$\frac{2}{2}$

س

- $\min$

L $\sum_{0} I$

능

임ำ

- No-

- 20

- NiDz

디 mIFO

$z$ o00?

แ= mกับง

马ु

-

IION

ZZI

इ जீய!

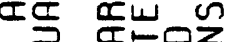

๙ 진

山a

$=0$ UL

ㅁ 늠

Bं 픙

는 두응

○ वozz

나 중-

o 뚱

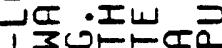
जCZ匹ー上E 0 س

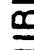

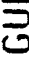

0

4 


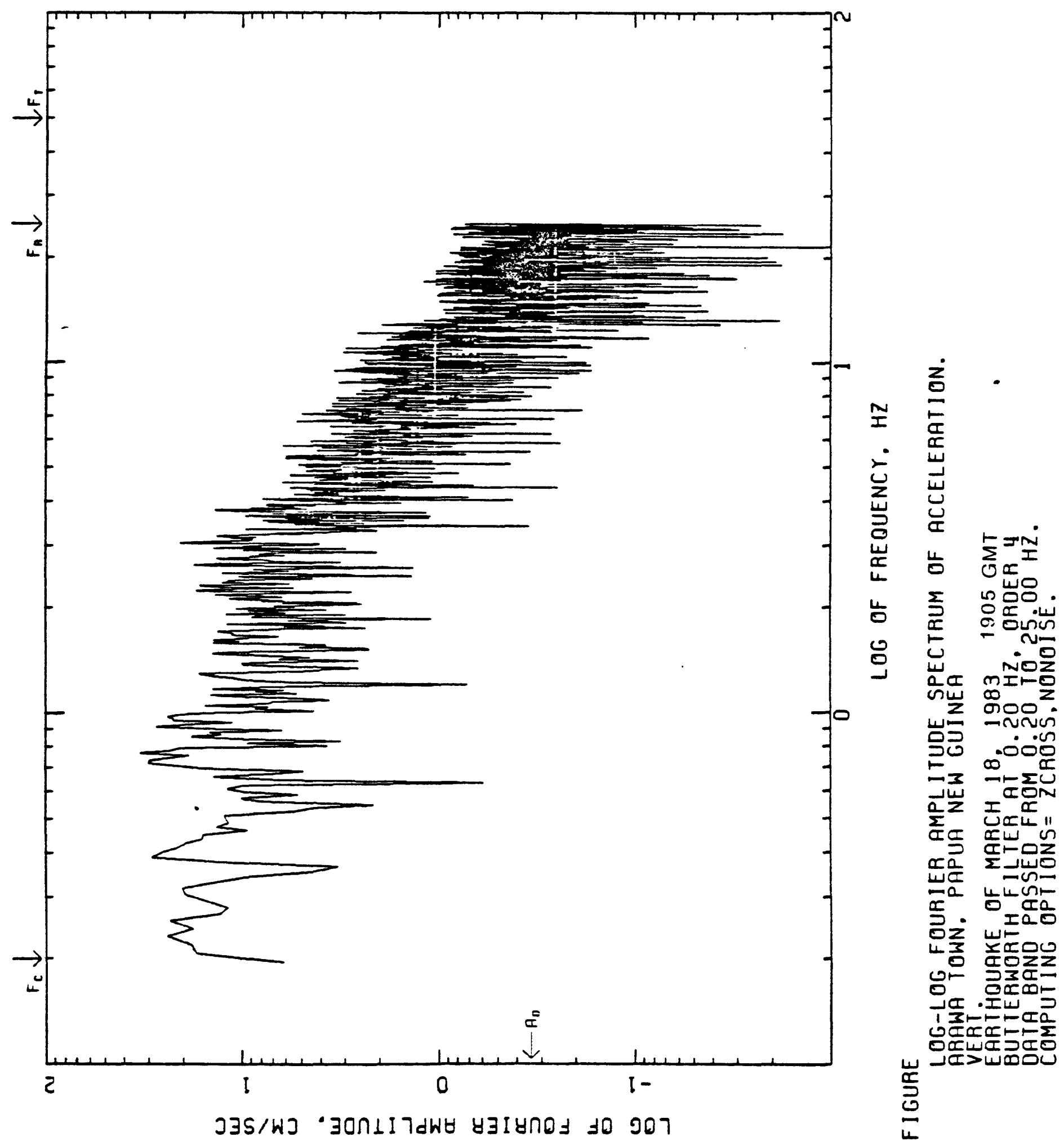



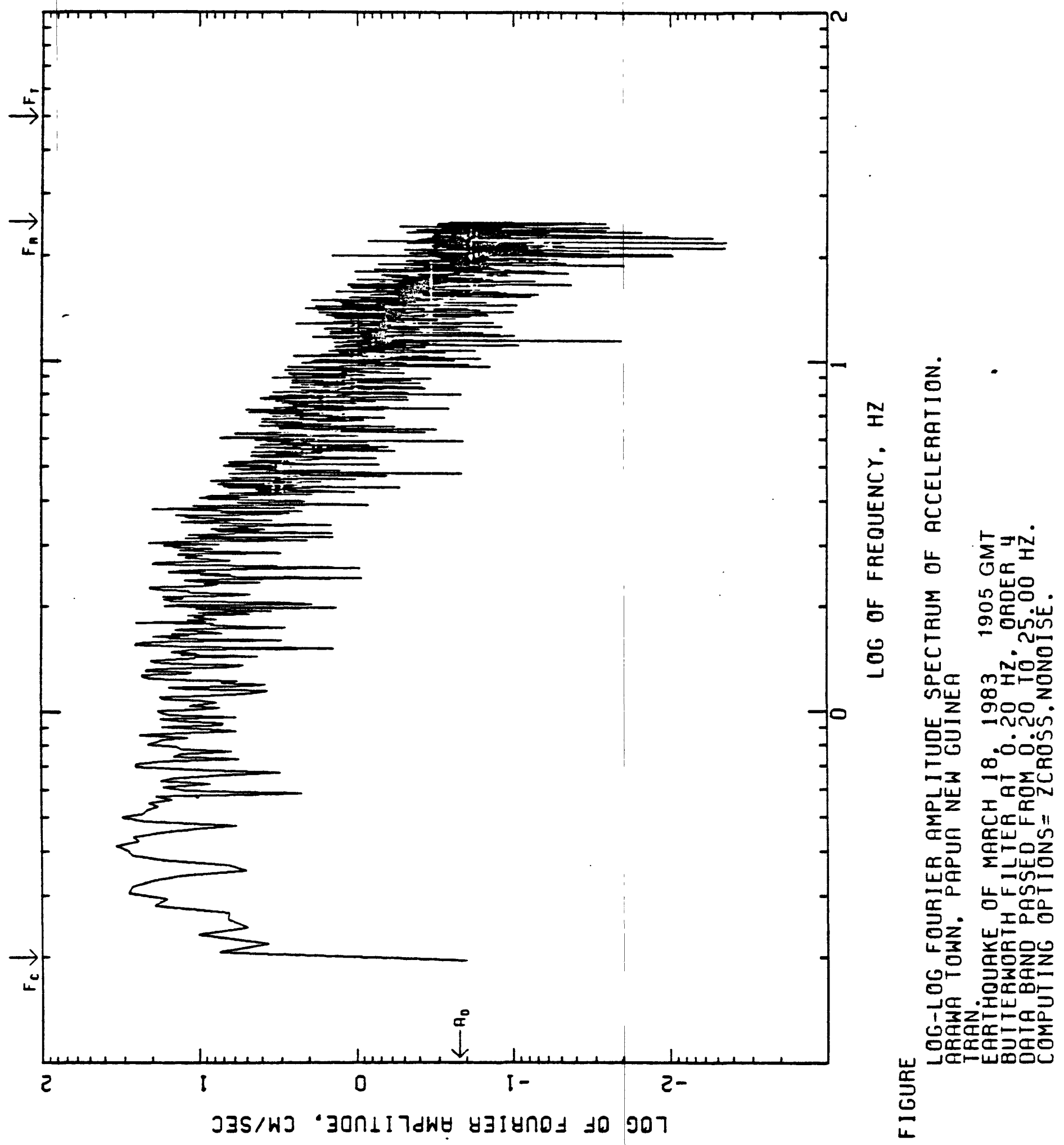


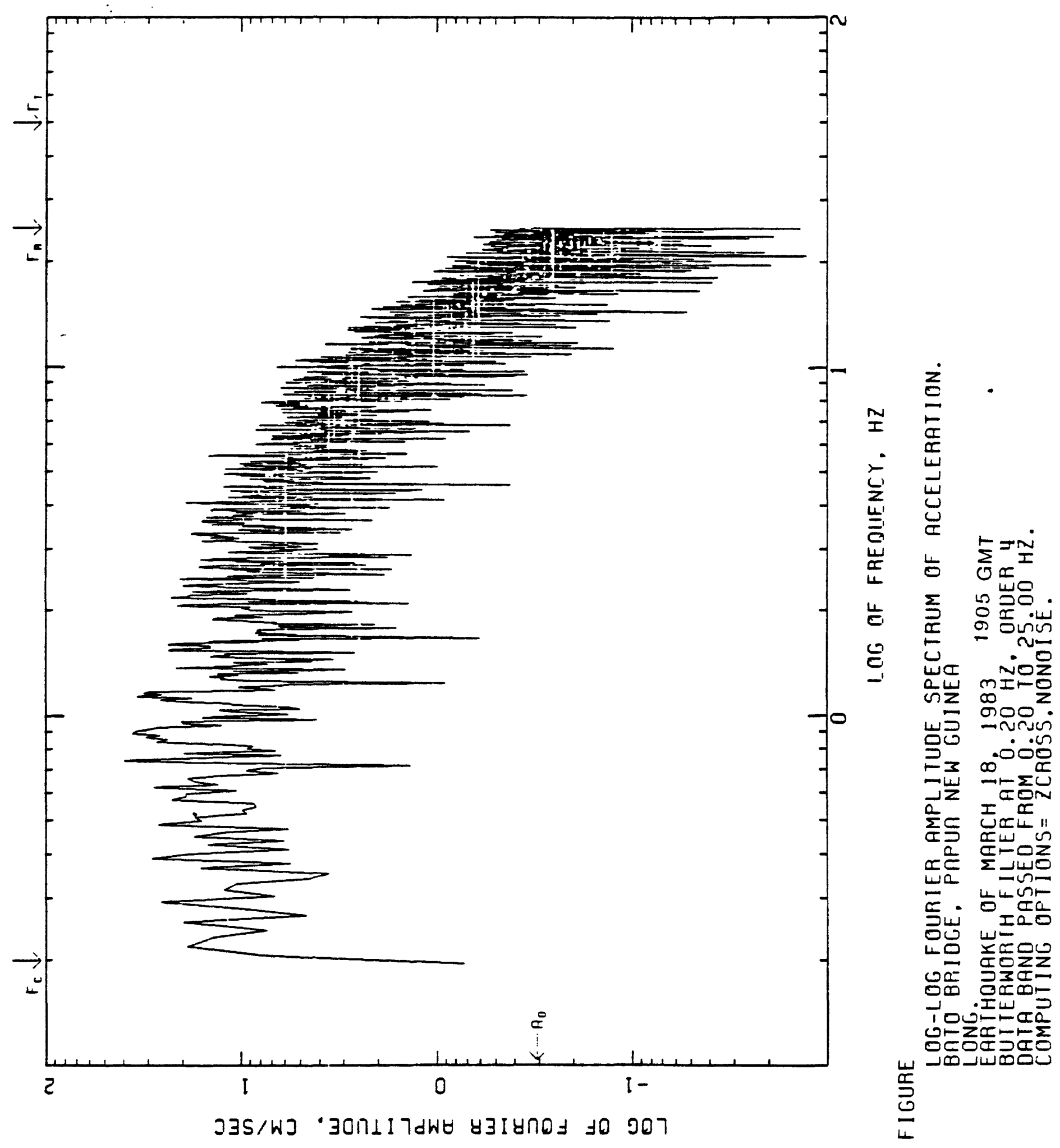



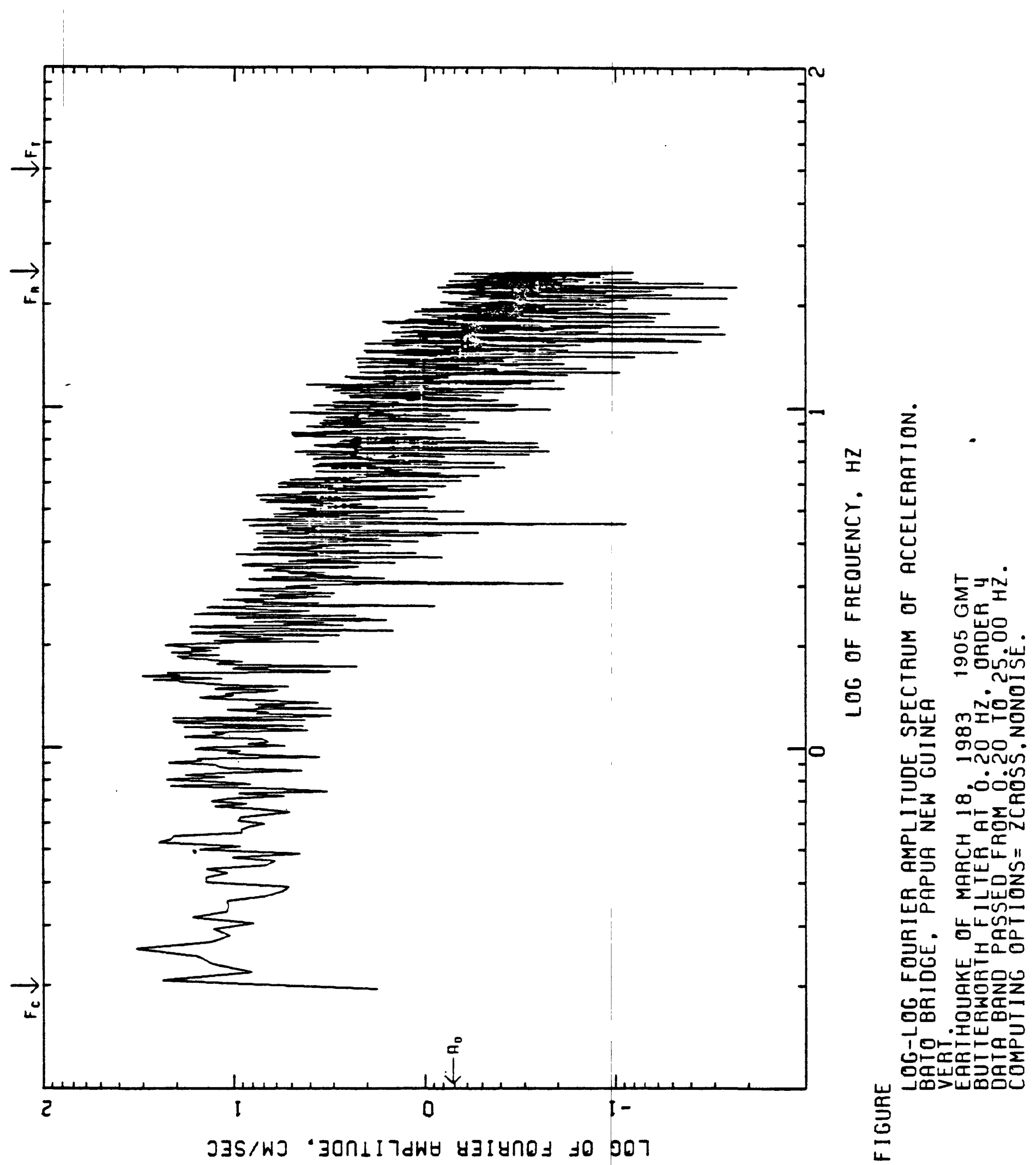

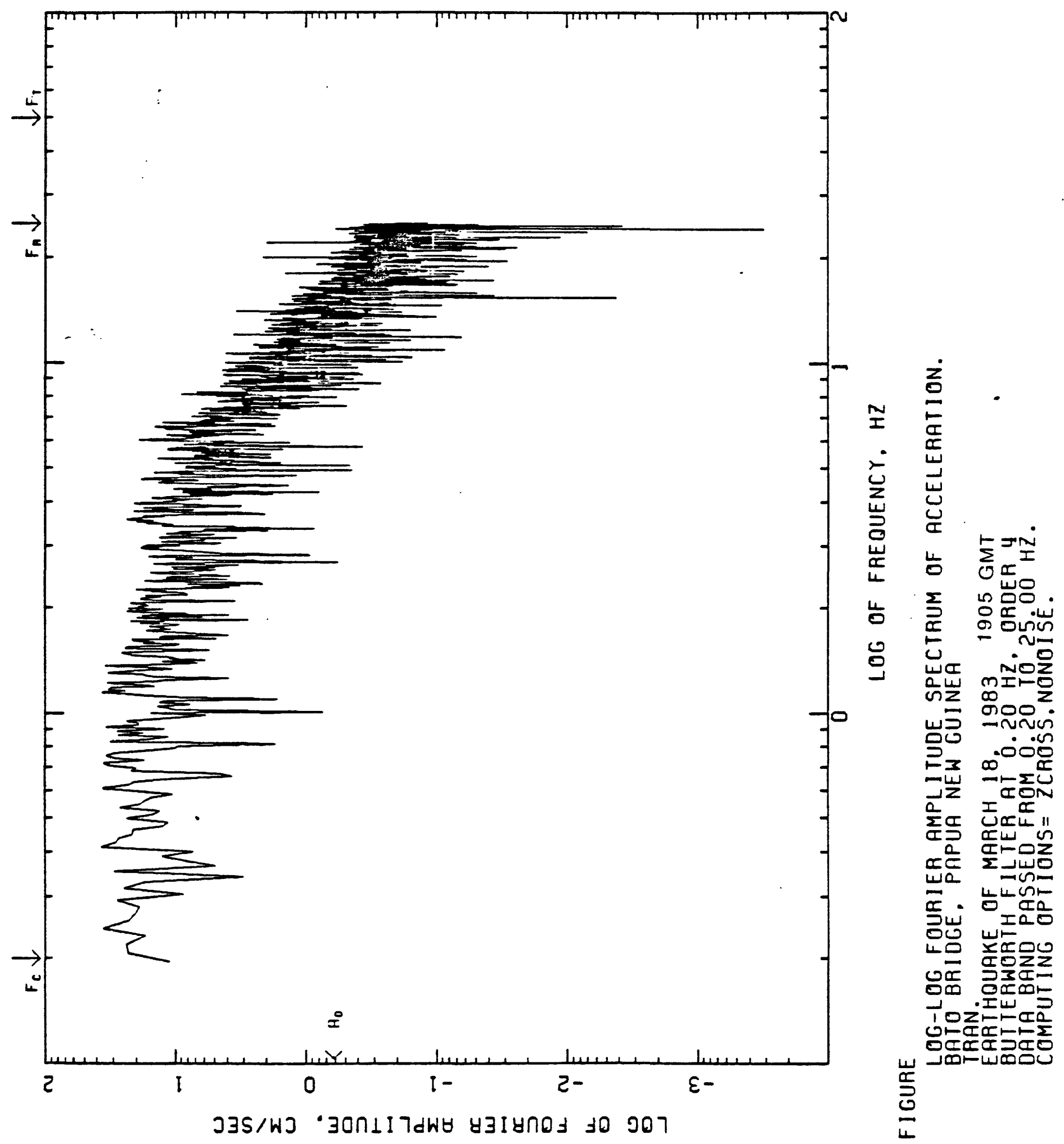

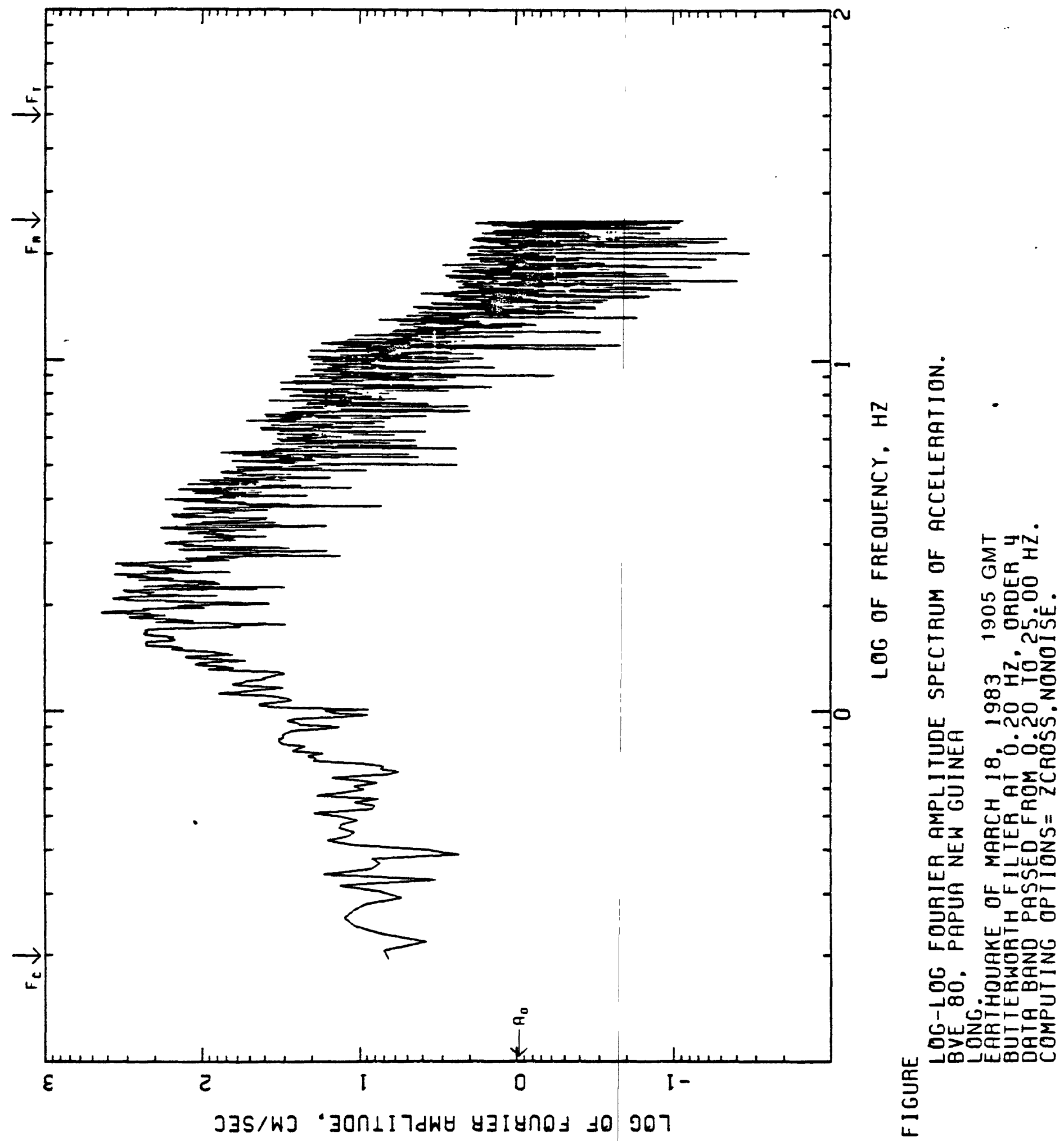


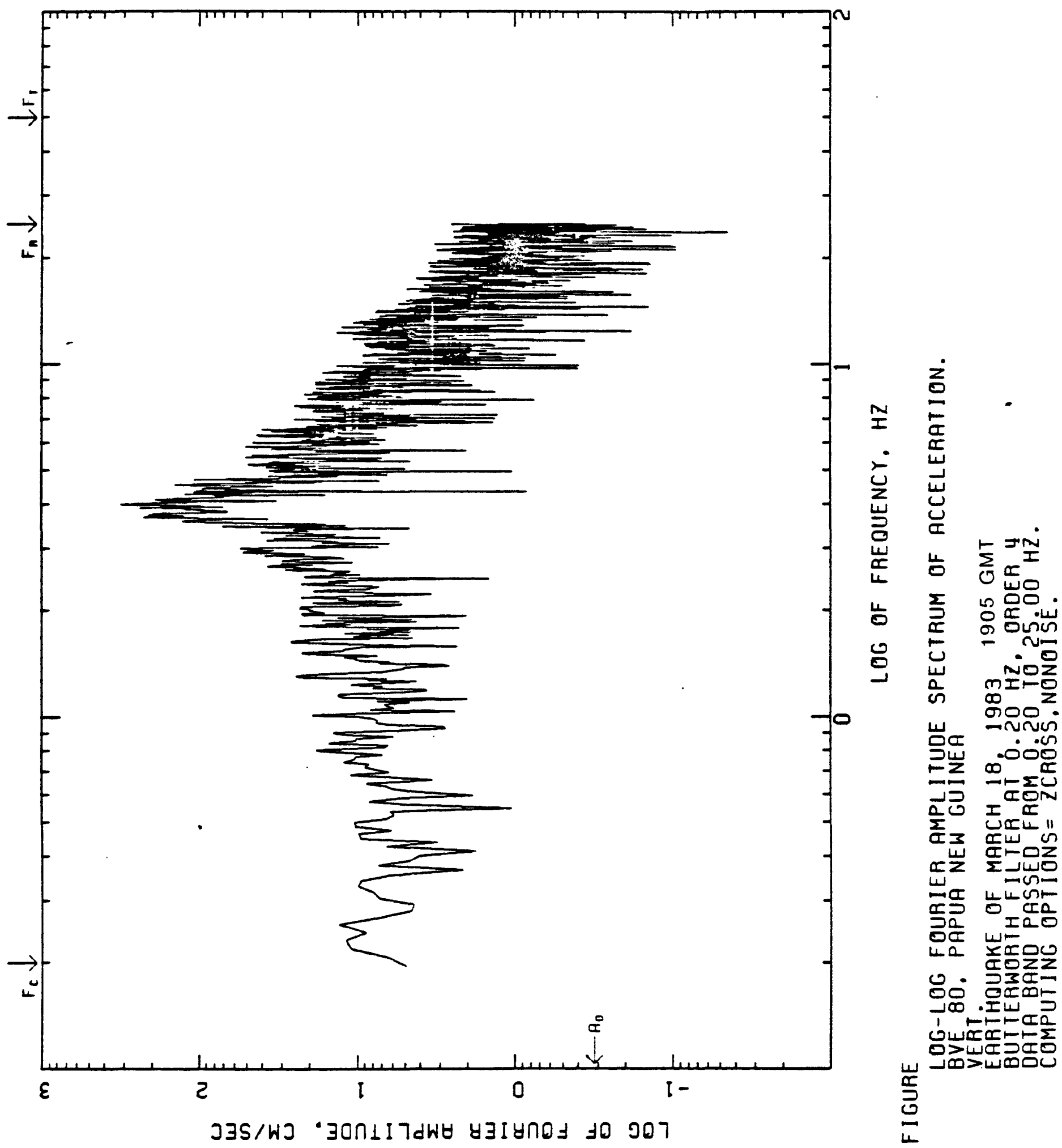




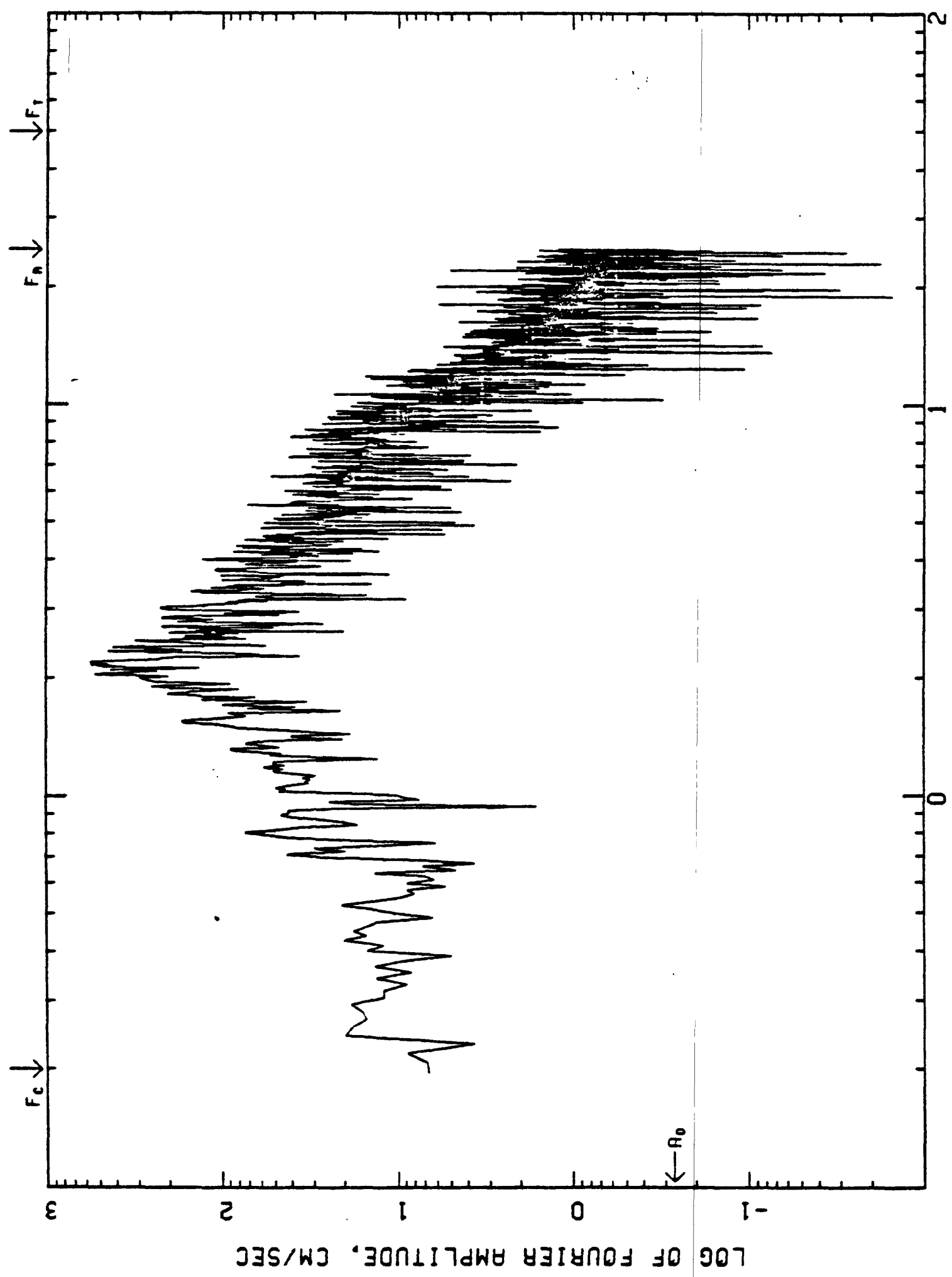

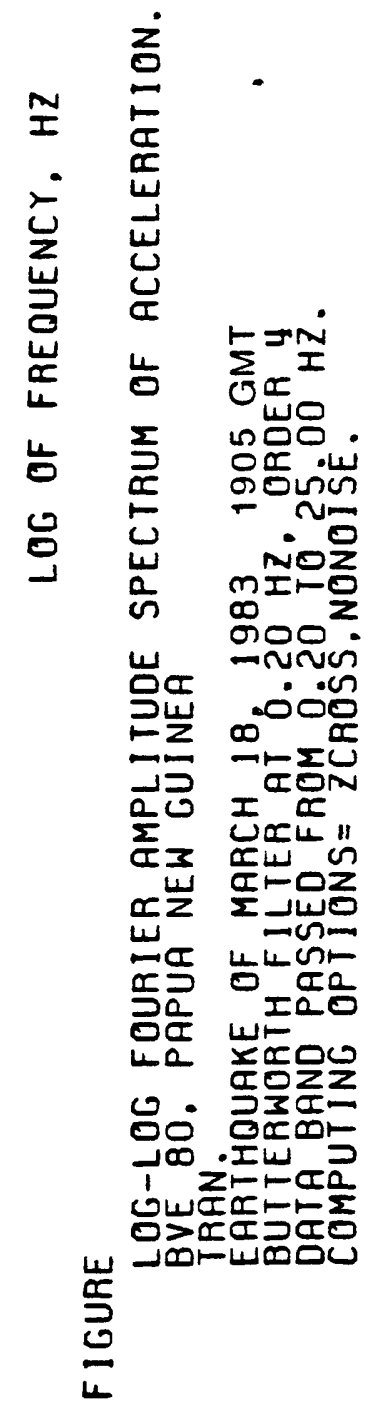


Appendix II

Current Lists of Processed Records 


\section{ripengis: is \\ CURRENT LISTS OF PROCESSED RECORDS}

TABLE 1. Chronological list of events and associated reports describing the existence, processing, analysis, and availability of digital data on tape from NOAA, or from the National Strong Motion Data Center in Menlo Park. USGS processing of records from the USGS permanent network of strong-motion accelerographs and associated networks. Strong motion data from earthquakes 1978* and later.

\begin{tabular}{|c|c|c|}
\hline Date \& Time (Gmt) & Earthquake & Reference (see attached list) \\
\hline January 1, 1975; 0355 & Southern Alaska; & $\begin{array}{l}\text { OFR 86-191 } \\
\text { (Silverstein, Braay, } \\
\text { Mork, 1986b) }\end{array}$ \\
\hline March 25, 1978; & $\begin{array}{l}\text { Coyote Dam, } \\
\text { California }\end{array}$ & $\begin{array}{l}\text { OFR 83-166 (Brady \& Perez, } \\
\text { 1983) }\end{array}$ \\
\hline $\begin{array}{l}\text { August } 27,1978 \text { and two } \\
\text { later shocks; }\end{array}$ & $\begin{array}{l}\text { Monticello Dam, } \\
\text { Jenkinsville, South } \\
\text { Carolina; }\end{array}$ & $\begin{array}{l}\text { OFR } 81-0448 \text { (Brady \& } \\
\text { others, 1981) }\end{array}$ \\
\hline August 6, 1979; & $\begin{array}{l}\text { Coyote Lake, } \\
\text { California }\end{array}$ & $\begin{array}{l}\text { OFR 81-42 (Brady \& others, } \\
1980)\end{array}$ \\
\hline October 15, 1979; & $\begin{array}{l}\text { Imperial Valley, } \\
\text { California; }\end{array}$ & $\begin{array}{l}\text { OFR 80-703 (Brady, Perez \& } \\
\text { Mork, 1980) }\end{array}$ \\
\hline October 15, 1979; & $\begin{array}{l}\text { Imperial valley, } \\
\text { California; }\end{array}$ & OFR 82-183 (Perez, 1982) \\
\hline $\begin{array}{l}\text { October 15, 1979; } \\
2317: 41,2318: 20,2318: 40\end{array}$ & $\begin{array}{l}\text { Imperial Valley } \\
\text { California aftershock }\end{array}$ & $\begin{array}{l}\text { OFR } 86-\text { (in preparation) } \\
\text { (Brady, Mork, Silverstein) }\end{array}$ \\
\hline October 16, 1979, 0706; & $\begin{array}{l}\text { Monticello Dam, } \\
\text { Jenkinsville, } \\
\text { South Carolina; }\end{array}$ & $\begin{array}{l}\text { OFR 81-1241 (Mork \& Brady, } \\
\text { 1981) }\end{array}$ \\
\hline $\begin{array}{l}\text { December 13, 1981 and } \\
\text { March 18, 1983; }\end{array}$ & Solomon Is lands; & $\begin{array}{l}\text { OFR } 86-264 \\
\text { (Silverstein, Brady, Mork, } \\
\text { 1986a) }\end{array}$ \\
\hline $\begin{array}{l}\text { February } 13,14, \\
\text { and } 23,1983 ;\end{array}$ & Monasavu Dam, Fiji; & $\begin{array}{l}\text { OFR } 85-375 \text { isilverstein, } \\
\text { 1985a) }\end{array}$ \\
\hline May 2 and May 9, 1983; & Coalinga, California; & $\begin{array}{l}\text { OFR } 84-626 \text { (Maley \& } \\
\text { others, } 1984 \text { ) }\end{array}$ \\
\hline JuTy 9, 1983; 0740; & Coalinga, California; & $\begin{array}{l}\text { OFR } 85-584 \text { (Silverstein, } \\
\text { 1985b) }\end{array}$ \\
\hline July 22, 1983; 0239; & Coalinga, California; & $\begin{array}{l}\text { OFR } 85-250 \text { (Silverstein and } \\
\text { Brady, 1985) }\end{array}$ \\
\hline
\end{tabular}

*With inclusion of isolated earlier events recently processed. 
TABLE 1. Chronological list of events and associated reports (continued)

\begin{tabular}{lll}
\hline Date \& Time (Gmt) & Earthquake & Reference (see attached list) \\
\hline April 24, 1984; & $\begin{array}{l}\text { Morgan Hill, } \\
\text { California; }\end{array}$ & $\begin{array}{l}\text { OFR 84-498, Vol I and II } \\
\text { (Compiled by Seena Hoose) }\end{array}$ \\
$\begin{array}{l}\text { December 23, 1985; 0516 GMT Northwest Territories, } \\
\text { and Nov. 9, Dec. 23, Dec 25 Canada }\end{array}$ & $\begin{array}{l}\text { OFR 86- } \\
\text { others, 1986) }\end{array}$ \\
January 26, 1986; 1920 GMT Hollister, & $\begin{array}{l}\text { OFR 86-156, (Brady and } \\
\text { California }\end{array}$
\end{tabular}


January 1, 1975; 0355; southern Alaska; OFR 86-191

Records (4): Anchorage, $500 \mathrm{~W}$. Third St., Basement

Anchorage, Alaskan Methodist University

Anchorage, Government Hospital

Talkeetna, FAA-VOR Building

March 25, 1978; Coyote Dam, California; OFR 83-166.

Records (3): Coyote Dam, Ukiah, California: abutment, toe, crest.

August 27, 1978, 1023 and 2 later shocks; Monticello Dam, South Carolina, OFR 81-0448.

Records (3): Jenkinsville, S.C. Monticello Dam

Shared abutment. (center crest)

August 27, 1978, 1023 GMT

Two later unidentified events

August 6, 1979, Coyote Lake, California; OFR 81-42

Records (6): Coyote Creek, San Martin, California

Gilroy Array: Station 6, San Ysidro, California

Gilroy Array: Station 4, San Ysidro School, California

Gilroy Array: Station 3, Sewage Treatment Plant, California

Gilroy Array: Station 2, Mission Trails Motel, California

Gilroy Array: Station 1, Gavilan College, California

October 15, 1979, 2317; The Imperial Valley Earthquake; OFR 80-703.

Records (22): El Centro Array 7, Imperial Valley College, California

El Centro Array 6, Huston Road

El Centro, Bonds Corner, Hiways 98 \& 115

El Centro Array 8, Cruickshank Road

El Centro Array 5, James Road

El Centro Differential Array

El Centro Array 4, Anderson Road

Brawley, Brawley Municipal Airport

Holtville, California, Holtville Post Office

El Centro Array 10, Keystone Road

Calexico, California, Calexico Fire Station

El Centro Array 11, McCabe School

El Centro Array 3, Pine Union School

Parachute Test Facility

El Centro Array 2, Keystone Road

El Centro Array 12, Brockman Road

Calipatria, California, Calipatria Fire Station

El Centro Array 13, Strobel Residence

El Centro Array 1, Borchard Ranch

Superstition Mountain, California

Plaster City, California, Storehouse

Coachella Canal Number 4, California 
TABLE 2. Processed records in each report. (continued)

October 15, 1979, 2317:41; Imperial Valley Aftershocks; OFR 86-

Records (6) El Centro Array 5, James Road

E1 Centro Array 6, Huston Road

El Centro Array 7, Imperial Valley College

El Centro Array 8, Cruickshank Road

El Centro Array 9, Commercial Ave.

El Centro Differential Array

October 15, 1979, 2318:20; Imperial Valley Aftershocks; OFR 86-

Records (6) El Centro Array 5, James Road

El Centro Array 6, Huston Road

El Centro Array 7, Imperial Valley College

El Centro Array 8, Cruickshank Road

El Centro Array 9, Commercial Ave.

El Centro Differential Array

Octoder 15, 1979, 2318:40; Imperial Valley Aftershock; OFR 86-

Records (7) El Centro Array 6, Huston Road

El Centro Array 7, Imperial Valley College

El Centro Array 8, Cruickshank Road

El Centro Array 9, Commercial Ave.

El Centro Differential Array

Bonds Corner, Highways 115 \& 98

Holtville Post Office

October 15, 1979; The Imperial Valley, California; OFR 82-183;

Records (22): This report contains the time-dependent response spectrum

plots for the same records as in OFR 80-703, above.

October 16, 1979, 0706 GMT, Monticello Dam, South Carolina, OFR 81-1214.

Records (1): Jenkinsville, South Carolina, Monticello Dam

shared abutment (center crest)

December 13, 1981 and March 18, 1983; Solomon Islands, OFR 86-264

Records (5): Dec. 13, 1981, 0129 GMT: 460 Beach, Panguna Mine,

Dec. 13, 1981, 1324 GMT: Bougainville Island.

March 18, 1983: Arawa Town

Bato Bridge

BVE80, Panguna Mine.

February 13, 14, and 23, 1983; Monasavu Dam, Fiji; OFR 85-375

Records (3): Feb 13, 14, 23, 1983: Monasavu Dam. 
TABLE 2. Processed records in each report. (continued)

May 2 and May 9, 1983; Coalinga, California; OFR 84-625.

Records (13): May 2, 1983, 2342 UTC:

Pleasant Valley Pump Plant: switchyard, basement

May 9, 1983, 0249 UTC

Anticline Ridge: freefield and pad

Burnett Construction

0il City

0il Fields Fire Station

Palmer Avenue

Skunk Hollow

Pleasant Valley Pump Plant: switchyard, basement,

lst floor, roof

July 9, 1983; 0740; Coalinga, California; OFR 85-584

Recoras (7): Anticline Ridge: freefield and pad

Burnett Construction

0i) City

0il Fields Fire Station: freefield and pad

Palmer Avenue

Skunk Hollow

Transmitter $\mathrm{Hill}$

July 22, 1983; 0239; Coalinga, California; OFR 85-250

Records (12): Anticline Ridge: pad site

Burnett Construction

0il City

0il Fields Fire Station: freefield and pad

Palmer Avenue

Pleasant Valley Pump Plant: Ist floor, basement, roof,

Skunk Hollow

switchyard, freefield

Transmitter $\mathrm{Hill}$

April 24, 1984; Morgan Hill, California; OFR 84-498B, Vol. II.

Records (11): Anderson Dam: downstream, crest

Hollister City Hall Annex

Hollister Differential Array

San Justo Damsite: right abutment, left abutment

San Jose 101/280/680 bridge

Hollister Differential Array No. 1, 3, 4, 5

December 23, 1985; with foreshock and aftershocks; Northwest Territories, Canada; OFR 86-

Records (6): $\overline{\text { Nov. }}$ 9, 1985; 0446 GMT: Nahanni Site 2

Dec. 23, 1985; 0516 GMT: Nahanni Sites 1, 2, 3

Dec. 23, 1985; 0548 GMT: Nahanni Site 1

Dec. 25, 1985; 1543 GMT: Nahanni Site 3

January 26, 1986; Hollister, California; OFR 86-156

Records (5): Hollister Digital Differential Array, Stations 1, 3, 4, 5, 6 


\section{References}

Brady, A. G., Etheredge, E. C., Maley, R. P., Mork, P. N., Silverstein, B. L., Johnson, D. A., Acosta, A. V., Forshee, R. D., and Salsman, M. J., 1986; Preliminary report on records from the USGS-maintained strong-motion network in the Hollister area, January 26, 1986. USGS Open-File Report $86-156$.

Brady, A. G., Mork, P. N., and Fletcher, J. P., 1981. Processed accelerograms from Monticello Dam, South Carolina, 27 August 1978, and two later shocks. USGS Open-File Report 81-0448, 35 pp.

Brady, A. G., Mork, P. N., and Silverstein, B. L., 1986, Processed strongmotion records from the 2317:41,2318:20 and 2318:40 aftershocks of the October 15, 1979, 2613:53 GMT earthquake; Imperial Valley, California. USGS Open File-Report 86, in preparation.

Brady, A. G., Mork, P. N., Perez, Virgilio, and Porter, L. D., 1980, Processed data from the Gilroy Array and Coyote Creek records, Coyote Lake, California Earthquake 6 August 1979; USGS Open-File Report 81-42, 171 pp.

Brady, A. G., Perez, Virgilio, and Mork, P. N., 1980. The Imperial Valley earthquake, October 15, 1979. Digitization and processing of accelerograph records. Open-File Report 80-703, 309 pp.

Brady, A. G. and Perez, Virgilio, 1983, Processed accelerograms from Coyote Dam, California, March 25, 1978: USGS Open-File Report 83-166 82 pp.

Brady, A. G., Porcella, R. L., Bycroft, G. N., Etheredge, E. C., Mork, P. N., Silverstein, B., and Shakal, A. F., 1984, Strong-motion results from the main shock of April 24, 1984, in The Morgan Hill, California earthquake of April 24, 1984, (S. Hoose, compiler), USGS Open-File Report 84-498A, V. I, pp. 18-26, and V. II, $103 \mathrm{pp}$.

Maley, R. P., Etheredge, E. C., Johnson, D. A., Switzer, J. C., Mork, P. N., and Brady, A. G., 1984, Strong motion data recorded near Coalinga, California (May 2, 1983) and processed data from May 2 and May 9, 1983: USGS Open-File Report 84-626, 258 pp.

Mork, P. N. and Brady, A. G., 1981, Processed accelerogram from Monticello Dam, Jenk insville, South Carolina, 16 October 1979, 0706 GMT: USGS Open-File Report 81-1214, 20 pp.

Perez, Virgilio, 1982, The Imperial Valley, California earthquake, October 15, 1979; time dependent response spectrum plots: USGS Open-File Report 82-183, 96 pp.

Silverstein, Barry, 1985a, Processed strong-motion records from Monasavu Dam, Fiji; Earthquakes of February 13, 14, and 23, 1983: USGS Open-File Report 85-375, $58 \mathrm{pp}$.

, 1985b, Processed strong-motion records from the Coalinga, California aftershock of July 9, 1983; 0740 GMT: USGS Open-File Report 85-584, 169 pp. 
Silverstein, B. and Brady, A. G., 1985, Processed strong-motion records from the Coalinga, California, aftershock of July 22, 1983, 0239 GMT: USGS Open-File Report 85-250, 229 pp.

Silverstein, B. L., Brady, A. G., and Mork, P. N., 1986a, Processed strongmotion records from Bougainville Is land, Papua New Guinea; earthquakes of December 13, 1981 and March 18, 1983: USGS Open-File Report 86-264.

1986b, Processed strong-motion records from the southern Alaska earthquake of January 1, 1975; 0355 GMT: USGS Open-File Report 86-191.

Weichert, D. H., P. N. Mork, and others. The November and December, 1985, Earthquakes in the Nahanni region of the Northwest Territories, Canada; in preparation. 